$$
\begin{aligned}
& \text { RECEIVED } \\
& \text { MAR } 03 \text { 1997 } \\
& \text { OSTI }
\end{aligned}
$$

\title{
MODIFICATIONS OF ALPHA PROCESSING SOFTWARE TO IMPROVE CALCULATION OF LIMITS FOR QUALITATIVE DETECTION
}

J. R. Kirkpatrick
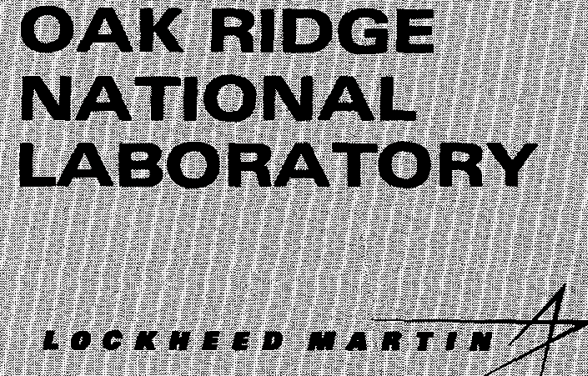$$
\text { (i) }
$$ 
This report has been reproduced directly from the best available copy.

Available to DOE and DOE contractors from the Office of Scientific and Technical Information, P. O. Box 62, Oak Ridge, TN 37831: prices available from (423) 576-8401, FTS 626-8401.

Available to the public from the National Technical Information Service, U.S. Department of Commerce, 5285 Port Royal Road, Springfield. VA 22161.

This report was prepared as an account of work sponsored by an agency of the United States Government. Neither the United States Government nor any agency thereof, nor any of their employees. makes any warranty, express or implied, or assumes any legal liability or responsibility for the accuracy, completeness, or usefulness of any information, apparatus, product. or process disclosed, or represents that its use would not infringe privately owned rights. Reference herein to any specific commercial product, process, or service by trade name. trademark, manufacturer, or otherwise. does not necessarily constitute or imply its endorsement, recommendation, or favoring by the United States Government or any agency thereof. The views and opinions of authors expressed herein do not necessarily state or reflect those of the United States Government of any agency thereof. 


\section{DISCLAIMER}

Portions of this document may be illegible electronic image products. Images are produced from the best available original document. 


\title{
MODIFICATIONS OF ALPHA PROCESSING SOFTWARE TO IMPROVE CALCULATION OF LIMITS FOR QUALITATIVE DETECTION
}

\author{
J. R. Kirkpatrick \\ Computational Physics and Engineering Division \\ Oak Ridge National Laboratory \\ This report \\ [Alpha Management Software (AMS)] \\ has been prepared for submission to
R. J. Schlossmann
Canberra Nuclear
Itasca, Illinois 60143 \\ 150 Spring Lake Drive \\ under License Agreement No. PLA-047
}

January 1997

\author{
Prepared by the \\ Analytical Services Organization \\ Oak Ridge, Tennessee 37831-8189 \\ managed by \\ LOCKHEED MARTIN ENERGY SYSTEMS, INC. \\ for the \\ U.S. DEPARTMENT OF ENERGY \\ under contract DE-AC05-84OR21400
}




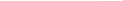




\section{CONTENTS}

Page

EXECUTIVE SUMMARY

$\mathrm{v}$

1. MODIFICATIONS OF ALPHA PROCESSING SOFTWARE TO IMPROVE CALCULATION OF LIMITS FOR QUALITATIVE DETECTION PART $1 \ldots \ldots \ldots \ldots \ldots \ldots \ldots \ldots \ldots$

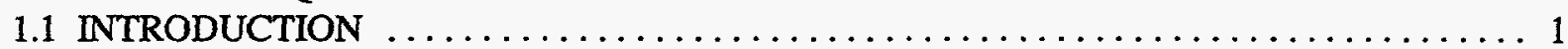

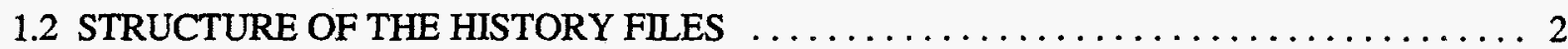

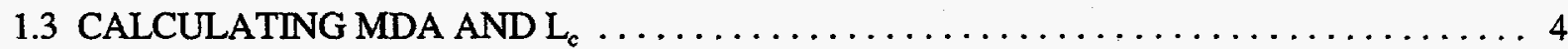

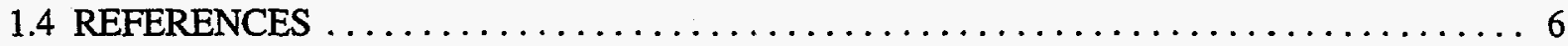

2. MODIFICATIONS OF ALPHA PROCESSING SOFTWARE TO IMPROVE CALCULATION

OF LIMITS FOR QUALITATIVE DETECTION PART $2 \ldots \ldots \ldots \ldots \ldots \ldots \ldots \ldots \ldots, \ldots$

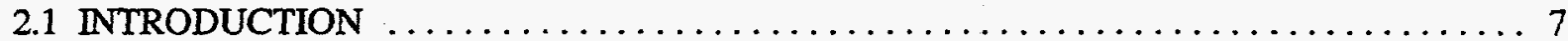

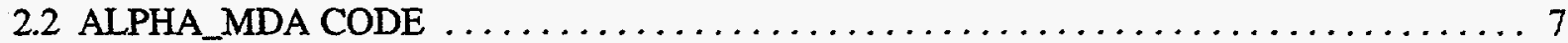

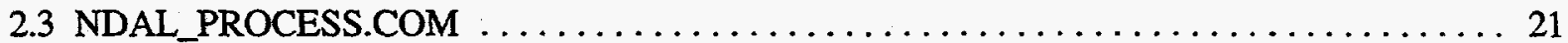

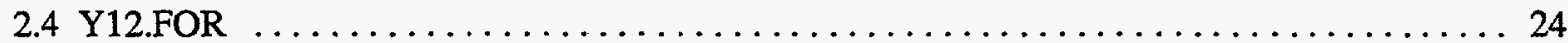

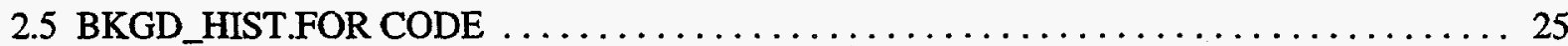

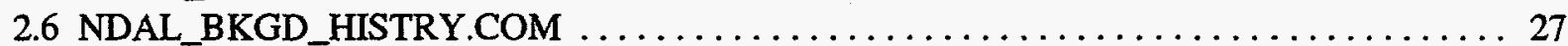

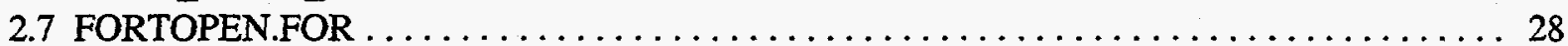

Appendix 2.A LISTING OF FORTRAN CODE ALPHA_MDA.FOR, FILE VERSION AS OF

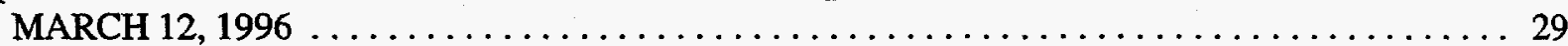

Appendix 2.B LISTING OF DCL FILE NDAL_PROCESS.COM, FILE VERSION AS OF

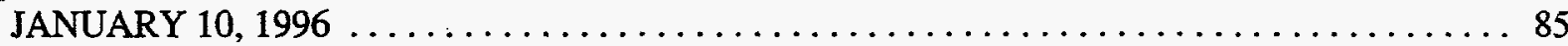

Appendix 2.C LISTING OF FORTRAN CODE Y12.FOR, FILE VERSION AS OF

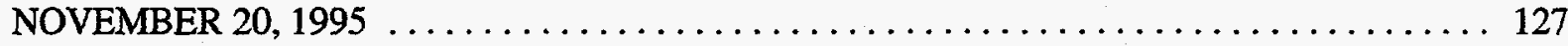

Appendix 2.D LISTING OF FORTRAN CODE BKGD_HIST.FOR, FILE VERSION AS OF

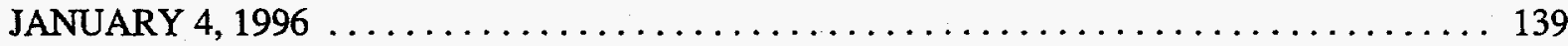

Appendix 2.E LISTING OF DCL FILE NDAL_BKGD_HISTRY.COM, FILE VERSION AS OF

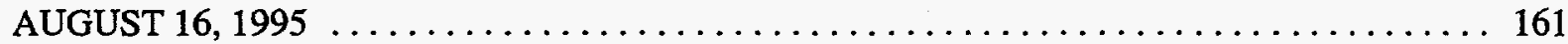

APpendix 2.F LISTING OF FORTRAN CODE FORTOPEN.FOR, FILE VERSION AS OF

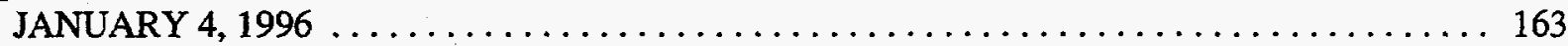

Appendix 2.G INSTRUCTIONS FOR DEALING WITH REAGENT BLANK SAMPLE EXCEEDS

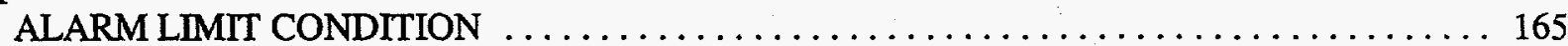

Appendix 2.H EFFECT OF TRUNCATION ERROR ON STANDARD DEVIATION $\ldots \ldots \ldots \ldots 167$ 


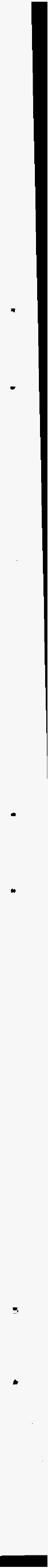




\section{EXECUTIVE SUMMARY}

The work described in this report was done for the Bioassay Counting Laboratory (BCL) of the Center of Excellence for Bioassay of the Analytical Services Organization at the Oak Ridge Y-12 Plant. BCL takes urine and fecal samples and tests for alpha radiation. An automated system, supplied by Canberra Industries, counts the activities in the samples and processes the results. The Canberra system includes hardware and software.

The managers of BCL want to improve the accuracy of the results they report to their final customers. The desired improvements are of particular interest to the managers of BCL because the levels of alphaemitting radionuclides in samples measured at $\mathrm{BCL}$ are usually so low that a significant fraction of the measured signal is due to background and to the reagent material used to extract the radioactive nuclides from the samples. Also, the background and reagent signals show a significant level of random variation. There are two aspects to improved accuracy. The first is improving the estimates of the signals from background and from the reagent. If one subtracts the background and reagent signals from the total signal in a measurement, the remainder is presumably due to actual sample activity. The second improvement desired is in the estimates of minimum values for detection. In a measurement process that is subject to statistical variations, there are signal levels that are so low that one may not be able to say with sufficient confidence that the signal is due to sample activity rather than to random variations. It is important that these minimum signal levels be calculated.

Statistical analyses of spectra have yielded signal levels that represent important thresholds. Two of these are $\mathrm{L}_{c}$, which is defined by Currie ${ }^{1}$ as "the net signal level (instrument response) above which an observed signal may be reliably recognized as detected," and $\mathrm{L}_{\mathrm{D}}$, also known as minimum detectable activity (MDA), which is defined as "the "true" net signal level which may be a priori expected to lead to detection."

The customers at BCL requested four major modifications of the software. First, they requested that activities from many background and reagent blank measurements be kept and that the standard deviations used for MDA be based on results from these multiple measurements. Next, the calculation for MDA was modified to include a sum of standard deviations of both backgrounds and reagent blanks. This second change replaced the previous scheme for calculating MDA, which used the number of counts from the most recent background measurement to estimate the standard deviation of the extraneous signal. Next, a calculation for $L_{c}$ was added. Last, an average reagent blank activity was subtracted from the sample activity in addition to the background to get an improved net signal. Formerly, only the background had been subtracted.

The requested software changes have been made and tested. The present report is in two parts. The first part describes what the modifications were supposed to accomplish. The second part describes the changes on a line-by-line basis. The second part includes listings of the changed software and discusses possible steps to correct a particular error condition. Last, the second part describes the effect of truncation errors on the standard deviations calculated from samples whose signals are very nearly the same. 


\section{MODIFICATIONS OF ALPHA PROCESSING SOFTWARE TO IMPROVE CALCULATION OF LIMITS FOR QUALITATIVE DETECTION PART 1}

\subsection{INTRODUCTION}

A major mission of the Bioassay Counting Laboratory (BCL) of the Center of Excellence for Bioassay (CEB) of the Analytical Services Organization at the Oak Ridge Y-12 Plant is determining the levels of radioactive nuclides in urine and fecal samples. The laboratory has an automated system, supplied by Canberra Industries, that includes hardware and software for measuring alpha emissions from the samples. The hardware produces alpha spectra that are passed to the software. The software identifies the nuclides in the samples and determines the levels of activity. If desired, some of the software can be modified at Lackheed Martin Energy Sysytems.

The levels of activity in almost all samples processed at $B C L$ are quite low. As a result, a significant part of the measured signal is due to counts from the background and from the reagent material. The most accurate determination of activity levels would require that the signals from both the background and the reagent be subtracted from the measured signal. The remainder of the signal would presumably be due to sample activity.

Another consequence of the low levels of activity is that the measured signals from reagent blanks and backgrounds are subject to statistical variation. The noise can be a significant fraction of the total signal. As a result, it is important to discriminate between signals that are high enough to be statistically significant and those that are so low that they may be caused solely by random variations. Statistical analyses have yielded expressions for signal levels that represent important thresholds. Two of these are $\mathrm{L}_{c}$, which is defined by Currie" $^{1}$ as "the net signal level (instrument response) above which an observed signal may be reliably recognized as detected," and $\mathrm{L}_{\mathrm{D}}$, also known as minimum detectable activity (MDA), which is defined as "the 'true' net signal level which may be a priori expected to lead to detection."

In the alpha processing software as it was previously written, the activity from the most recent background measurement for that counter was subtracted from the sample measured signal to get a first estimate of the net signal. Also, a first-order approximation of MDA was calculated using the square root of the background signal as an estimate for the standard deviation of the background. No calculation of $L_{c}$ was done. The customers at CEB wanted the new software to do three additional things. First, they wanted a reagent blank signal to be subtracted from the sample signal, in addition to the background signal that was already being subtracted. To improve the statistics, they wanted this reagent blank signal to be an average from several reagent blanks. Second, they wanted a more accurate value for MDA to be calculated by using a combination of standard deviations from several background measurements and several reagent blanks. This change represents two improvements in MDA: (1) a better approximation of the signal that would be measured in the absence of sample activity using the combination of reagent blank and background standard deviations rather than just the background and (2) more accurate statistics, made possible by using actual standard deviations calculated from several measurements rather than approximating the standard deviation from the results of a single measurement. The third improvement desired was a calculation of $L_{c}$, to be done using the same background and reagent blank standard deviations as those used for the improved MDA.

A number of steps were needed to make the desired improvements. First, it was necessary to keep "histories" of activities from previous background and reagent blank measurements. Routines were written to create "history" disk files, to add results from most recent measurements to these files, and to remove old results, as needed. Other routines were created to calculate averaged reagent blank activities and to calculate standard deviations for both backgrounds and reagent blanks. Next, code was written to subtract an average reagent blank activity from the sample activity to get a net signal. Then, the calculation for MDA was 
modified to include a combination of standard deviations of both background and reagent blanks. Last, a calculation for $L_{c}$ was added.

One of the desired features of the modifications was that the software be changed as little as possible. A second desired feature was that the code modifications be concentrated in certain areas and be plainly marked. Further, it was desired that the modified code be usable at all locations that have the Canberra software without changing the results for any users except BCL. After the current software was studied and the mission of the changes clearly outlined, it was determined that the changes could be limited to three parts of the software. A few small changes were made in the DCL file NDAL_PROCESS.COM, which runs the software to process the samples. A small number of changes were made in the FORTRAN code Y12.FOR, which creates the flat file that contains results to be reported to the final customer. Note that, in June 1995, R. J. Schlossmann of Canberra changed the NDAL_PROCESS.COM file so that the executable file for the Y12.FOR code is now called using an alias from the LOGIN.COM file called FLAT_FILE_OUTPUT. In other words, the FORTRAN and executables are still called Y12.FOR and Y12.EXE, but the execution uses this alias. Most of the changes that needed to be made were done in the FORTRAN code ALPHA_MDA.FOR. After the initial modifications were done, it was noted that not all the background samples were being included in the histories. To correct this omission, it was necessary to copy the background programming from ALPHA_MDA.FOR into a separate program called BKGD_HIST.FOR and make some further modifications. BKGD_HIST.FOR is executed from a DCL file called NDAL_BKGD_HISTRY.COM, which is executed in a different portion of the NDAL_PROCESS.COM file from ALPHA_MDA.FOR.

The software changes have been made and tested and are being used in normal operation. The present report describes the rationale behind the changes. The second part of the report describes the changes themselves on a line-by-line basis. The second part also includes listings of the changed software and discussion of possible steps to take to correct a particular error condition. Last, the second part contains an analysis of the effect of truncation errors on the standard deviations of samples whose variability is small.

\subsection{STRUCTURE OF THE HISTORY FILES}

There is a blank history file for each matrix and each library. The name of each file is "dub1:[alpha.alusr.blnk]matrix_controllib_blank.his," where "matrix" is a set of characters identifying the material in which the sample was embedded (normally "fecal" or "urine") and "controllib" is the identifier of the library ("UU," "PU," "AM," etc.). In Canberra documentation, the word "library" is used to indicate a set of boundaries that give the regions in alpha energy where the nuclides one is seeking occur. Given these regions and other data about the expected nuclides, the software can determine how much material occurs in each of the regions and eventually determine the activity of each nuclide. The blank history files contain results from up to 20 separate reagent blank measurements for that detector and that library. The most recent measurement is first, followed by the next most recent, and so on. When the reagent blank history file maintenance routines in ALPHA_MDA.FOR determine that the sample being processed is a reagent blank and is not one that is already in the history file, this new blank is added to the file. If there are already 20 blanks in the file, the oldest is removed. The first record in the blank history file gives the batch and sample I.D.'s of the most recent blank sample and the date and time the sample was added to the history file. The last 27 characters of the name of the history file are written at the end of this record. That information is not read when the ALPHA_MDA.FOR code reads the sample history data, but is added so that someone who prints the history file or looks at it on the screen or with an editor can conveniently determine what file he is examining. The next records give the blank activity, six-sample averaged activity (the average is calculated using the most recent samples in the history up to 6), and the 20-sample standard deviation (the standard deviation is calculated using all the samples in the history up to 20 ) in units of $\mu \mathrm{Ci}$. There is a record for each 
nuclide in the sample. The pattern "act,average,std dev(uCi)" is written at the end of each of those records. That information is not read when the ALPHA_MDA.FOR code reads the sample history data, but is added so that someone who prints the file or looks at it on the screen or with an editor can conveniently determine what the entries on the record represent and what the units are. The rest of the file contains the same information for each of the remaining samples in the file.

A recent (January 1996) addition to the blank history area is the blank "archive". file. This file was added so that there would be a record of the blank history information for more than just the last 20 reagent blank samples. The name of each file is "dub1:[alpha.alusr.blnk]matrix_controllib_blank.ark." The structure of the data is identical to that in the blank history files with the ".his" extension. Whenever the ALPHA_MDA.FOR code adds another reagent blank sample to the ".his" history file, it also appends the lines of blank history information for that file to the ".ark" archive file. Thus, the archive files will continue to grow in size until they are transferred to some archival medium and deleted.

There is a background history file for each detector and each library. The name of each file is "dub1:[alpha.alusr.bkgd]chamber_controllib_bkgnd.his," where "chamber" is the detector number (from "001" to "999") and "controllib" is the identifier of the library ("UU," "PU," "AM," etc.). The meaning of "library" in Canberra documentation was explained above. The background history files contain results from up to 20 separate background measurements for that detector and that library. The most recent measurement is first, followed by the next most recent, and so on. When the background history file maintenance routines in ALPHA_MDA.FOR determine that the background specified for the sample being processed is not already in the history file, this new background is added to the file. If there were already 20 backgrounds in the file, the oldest is removed. The first record in the background history gives the batch and sample I.D.s of the most recent background sample, the live time (i.e., time duration of the measurement) for this sample, and the date and time the sample was added to the history file. The last 22 characters of the name of the history file are written at the end of this record. That information is not read when the ALPHA_MDA.FOR code reads the sample history data, but is rather added so that someone who prints the history file or looks at it on the screen or with an editor can conveniently determine what file he is examining. The next records give the background activity and the 20-sample standard deviation (the standard deviation is calculated using all the samples in the history up to 20) in units of counts/time. There is a record for each nuclide in the sample. The pattern "act,std $\operatorname{dev}($ counts/s)" is written at the end of each of those records. That information is not read when the ALPHA_MDA.FOR code reads the sample history data, but is added so that someone who prints the file or looks at it on the screen or with an editor can conveniently determine what the entries on the record represent and what the units are. The rest of the file contains the same information for each of the remaining samples in the file.

Some comments need to be made concerning the "live time" that is stored for each of the background samples in the background history files. At BCL, it is most common for background measurements to be made on the weekends and to be run for approximately $24 \mathrm{~h}$. Other measurements, including both reagent blanks and samples, are usually run for approximately $16.67 \mathrm{~h}$ (i.e., about $1000 \mathrm{~min}$ ). An advantage of the longer time for backgrounds is that the statistics for backgrounds are thereby improved. The background is caused by rather infrequent events that have a random distribution in time. The number of background counts for most nuclides is rather small and shows a large random variation from measurement to measurement. Running background measurements for $24 \mathrm{~h}$ rather than $16.67 \mathrm{~h}$ increases the number of background events, thereby lowering the variability in the background measurements. However, if one attempts to compare measured background counts to measured sample counts, the number of background counts is overstated because of the longer measurement time. For comparisons to be valid, both kinds of counts must be normalized to the same measurement time. When the Canberra software begins to process a reagent blank or a sample, the software determines which background is appropriate to that measurement. A background may have already been assigned to the sample. If not, the software assigns the most recent background measurement done for that detector. The software then uses the data from the assigned background sample to create a dummy background 
sample file. This dummy contains a background spectrum whose counts are those from the real background measurement, normalized to the live time of the sample being processed. In other words, the background sample counts are multiplied by the ratio of the sample live time to the background measurement time (in the usual case, this ratio is approximately 16.67/24), and the result is passed to the dummy background file. It is this dummy whose values are read by ALPHA_MDA.FOR for comparison with the reagent blanks or samples.

The steps leading to a normalized set of background counts described in the previous paragraph were in the Canberra software when the present report's author began working with it and have not been modified. There are two paths through which the results from a background measurement appear in the background history file. One path is that an execution of ALPHA_MDA.FOR determines that the background measurement does not appear in the history file and adds its results. In the modified ALPHA_MDA.FOR, the normalized background count values are passed to the background history file. The live time passed to the history files from ALPHA_MDA.FOR is that for the sample that was being processed when the particular background measurement was first entered into the history file. The alternate path for a background to appear in the history file is that an execution of BKGD_HIST.FOR determines that the background measurement does not appear in the history file and adds its results. In that case, the numbers of background counts passed to the history file represent actual counts without any normalization. The live time passed to the history files by BKGD_HIST.FOR is that of the background measurements.

\subsection{CALCULATING MDA AND $\mathrm{L}_{\mathrm{c}}$}

Currie ${ }^{1}$ shows how to calculate $L_{c}$ and MDA. A set of equations for $L_{c}$ and MDA are contained in the letter from M. M. Reichert to M. R. Prochownik . The equations in Reichert's letter represent Currie's methods as applied to alpha-emitting radionuclides. A further refinement of the derivation was presented in a FAX from L. A. Lewis to J. Webb ${ }^{b}$. Minor additional changes in notation were made by Lewis and Tucker and by Lewis. The expressions for MDA and $L_{c}$ are as follows:

$$
\begin{gathered}
\mathrm{MDA} \equiv 3 / \mathrm{ERTA}+4.65\left\{\left(s_{\text {background }} / \text { ERTA }\right)^{2}+s_{\text {interference }}{ }^{2}\right\}^{1 / 2}, \\
L_{c} \equiv 2.33\left\{\left(s_{\text {background }} / \text { ERTA }\right)^{2}+s_{\text {interference }}\right\}^{2},
\end{gathered}
$$

where $E$ is the detector efficiency, $R$ is the sample yield, $T$ is the duration of the measurement, and $A$ is the abundance of the nuclide. The notation is that $\mathrm{s}_{\text {background }}$ is the standard deviation of the background

${ }^{a}$ M. M. Reichert, Y-12 Analytical Services Organization, "Determination of Detection Limits for Alpha Emitting Radionuclides," letter to M. R. Prochownik, EG\&G Rocky Flats, Inc., September 1, 1994.

'L. A. Lewis, Y-12 Analytical Services Organization, "MDA Derivation," FAX to J. Webb, EG\&G Rocky Flants, Inc., November 7, 1994.

L. A. Lewis and W. O. Tucker, personal communication to J. R. Kirkpatrick, December 14, 1994.

'L. A. Lewis, personal communication to J. R. Kirkpatrick, December 29, 1994. 
measurements in units of counts. The division by ERTA is a conversion from counts to activity. The term $s_{\text {interference }}$ is the standard deviation of the reagent blank samples in units of activity (normally, the units are $\mu \mathrm{Ci}$ ).

There are values of MDA and $\mathrm{L}_{\mathrm{c}}$ for each detector, matrix (i.e., urine or fecal), and nuclide. Background measurements are done for each detector, but the background is independent of the matrix. The software sorts the background counts into the regions appropriate to the nuclides so that there is a background measurement for each nuclide. Reagent blank measurements are not done for every detector, but the different matrices require different reagents. Thus, there are separate reagent blank samples for each matrix. The software sorts the reagent blank counts into the regions appropriate to the nuclides so that there is a blank measurement for each nuclide. Some assumptions are necessary to combine the background and reagent blank measurements to get MDA and $\mathrm{L}_{\mathrm{c}}$ values for all the combinations of detector, matrix, and nuclide. A basic assumption is that the reagent blank activity minus the background activity from its own detector should be independent of the detector. The standard deviation of many measurements of this activity is $s_{\text {interference, }}$ which is dependent on the matrix and the nuclide. A second assumption is that the background counts for the nuclide divided by the ERTA of the detector, matrix, and nuclide of the sample currently being analyzed is the background activity for the nuclide. The background activity is independent of the matrix, but not of the detector. Thus, combining the standard deviations of backgrounds and reagent blanks as shown in the equations for MDA and $\mathrm{L}_{c}$ yields a result that is specific to each set of detector, matrix, and nuclide.

As was noted earlier, the average reagent blank activity used to correct the activity from a regular sample uses activities from the most recent up to six reagent blank samples. The number six was chosen by the customers at BCL, L. A. Lewis, and W. O. Tucker, and represents their judgment about how many samples are needed to provide a statistically valid result. If there are not results from six samples available in the history file, the average will be performed using as many samples as are available. If the reagent blank history file contains no sample results, then the average is assumed to be zero. Whenever a sample is corrected for the average reagent blank activity, the reagent activity subtracted is passed to the basic sample data file and stored in a spare data string (variable $d u m 3$ ). The average reagent blank activity is not subtracted from samples that are themselves reagent blanks. As was also noted in the previous paragraph, the standard deviation for the reagent blank activities for calculating MDA and $\mathrm{L}_{c}$ uses activities from the most recent up to 20 reagent blank samples. The number was chosen by the customers at BCL and represents their judgment about how many samples are needed to provide a statistically valid result. If results from 20 samples are not available in the history file, the standard deviation will be performed using as many samples as are available. However, if the reagent blank history file contains results from fewer than four blank samples, then the routine returns a zero value for the reagent blank standard deviation.

The calculation of standard deviations for backgrounds is almost identical to that for reagent blanks. Of course, no average sample activity is calculated for backgrounds. The only major difference between the reagent blank standard deviation calculation and that for the background happens if the background history file contains results from fewer than four background measurements. In that eventuality, the software reverts to the model used before these changes were made. That is, the background standard deviation is assumed to be the square root of the number of counts of the most recent background measurement for that detector.

\subsection{REFERENCES}

1. L. A. Currie, "Limits for Qualitative Detection and Quantitative Determination, Application to Radiochemistry," Anal. Chem. 40, 586-93 (March 1968). 


\section{MODIFICATIONS OF ALPHA PROCESSING SOFTWARE TO IMPROVE CALCULATION OF LIMITS FOR QUALITATIVE DETECTION \\ PART 2}

\subsection{INTRODUCTION}

This part of the report contains descriptions of the modifications to the FORTRAN codes ALPHA_MDA.FOR and Y12.FOR and to the DCL file NDAL_PROCESS.COM. Listings of the FORTRAN codes ALPHA_MDA.FOR, Y12.FOR, BKGD_HIST.FOR, and FORTOPEN.FOR; and of the DCL files NDAL_PROCESS.COM and NDAL_BKGD_HISTRY.COM follow as Appendixes 2.A, 2.C, 2.D, 2.F, 2.B, and 2.E, respectively). Appendix 2.G describes possible responses to having a reagent blank sample exceed the dpm alarm limit. Last, Appendix 2.H discusses the effect of truncation errors on the standard deviation of samples in a history file.

\subsection{ALPHA_MDA CODE}

In ALPHA_MDA.FOR, there are a modest number of changes in the main code. In addition, there are some very large new subroutines with several entries each that accomplish the creation and maintenance of the history files and the communication from the history files to the main routine. Last, there are some small new utility subroutines used by the history subroutines. The remainder of this section explains in some detail what is going on in each routine to make future maintenance of the code easier. A listing of the entire ALPHA_MDA.FOR file is included as Appendix 2.A. The line numbers shown at the first of each line in the appendix are referenced in the discussion of the code in this section. All additions and changes by Kirkpatrick are bracketed by comment cards starting with “jxk, which is Kirkpatrick's computer user ID.

\section{OPERATIONS IN MAIN ROUTINE}

A. Get value of flag y12_bioassay_output, which is used to determine whether any of these modifications will be executed. Lines $163,165$.

B. Set default for 2nd constant of MDA (lld_constant) to 3.0 rather than 2.71. This is done if y12_bioassay_output .eq. 'YES'.or. srs_bioassay(1:1).eq.' 'Y'. Lines 242 through 246.

C. Change header of message written after executing ALPHA_MDA to show that last two columns of message line are MDA and $\mathrm{L}_{c}$. This is done if $y 12$ _bioassay_output .eq. 'YES' .or. new_reagent_calc(1:1).eq. ' $Y$ '. Lines 598 through 606.

D. Check to see if y12_bioassay_output.eq. 'YES'.or. new_reagent_calc(1:1).eq.' $Y$ '. None of the next lines will be executed if this "if" statement is not true. Lines $634,651$.

1. Initialization of blank history information_call hblkini(debug,nbr,batch_id,sident,yield,time_str). Line 636.

2. Find background batch ID field ( $b k g b a t_{-} i d$ ) and truncate result to 24 rightmost characters. Lines 640 through 643.

3. Initialization of background history information-call hbkgini(debug,nbr,bkgbat_id,bkgident,time_str). Line 645.

4. Get dpm alarm threshold (dpm_alarm). Lines 648, 649. 


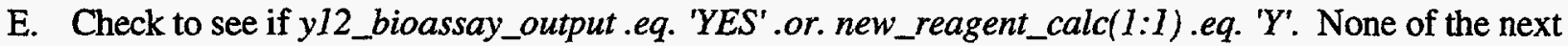
lines will be executed if this "if" statement is not true. Lines 678, 704.

1. Return blank averaged activity--call gtrbblk(xnumb,rbblnk, debug). Line 680.

a. Check to see if value returned $(r b b l n k)>-10^{25}$. Lines $683,703$.

(1) If so, the sample is not a reagent blank and there is a usable value of rbblnk.

(a) Subtract the averaged blank activity $(r b b l n k)$ from the sample activity to correct for blank activity, provided this is not the tracer nuclide (xnucl.ne. CER_nucl). Lines 689 through 691.

(b) Pack the averaged blank activity into spare string dum3 [call reinpak(rbblnk,dum3)] so that it can be stored in the sample data [by the $s=c a m \_$nnucl(c,xnumb,hlif,xnucl,sbhdr,hunits) after statement 9900 on line 975] for later recovery if need be. Line 701.

Note about order of operations: The six-sample averaged blank activity is subtracted from the sample activity at line 689. However, the subtraction will not be done for reagent blank samples. This is before the call to hblkinf at line 738 , in which the six-sample averaged blank activity is actually calculated. The operations in that order work successfully because reagent blank samples are processed before normal samples. The correction for six-sample blank activity at line 689 is not done for reagent blank samples because gtrbblk recognizes blank samples and returns rbblnk $<-10^{25}$, which the "if' statement at line 683 uses as a flag to know that rbblnk is not to be subtracted from the activity. For a reagent blank sample, the call to hblkinf at line 738 completes the calculation of the six-sample blank averaged activity. When a normal sample is processed, a six-sample averaged activity based upon the most recent reagent blank samples is available because the reagent blank samples were processed first.

F. Check to see if y12_bioassay_output .eq. 'YES'.or. new_reagent_calc(1:1) .eq. ' $Y$ '. Lines 714, 723.

1. If so, further process background history information to create 20-sample background standard deviation-call hbkginf(debug,xnumb,bkgcts,bkgtim,bkgbat_id,bkgident). Lines 719 through 722 .

G. Check to see if y12_bioassay_output.eq. 'YES'.or. new_reagent_calc(1:1) eq. ' $Y$ '. Lines $733,739$.

1. If so,

a. Convert weighted mean (wtmean) from real*8 to real*4 variable (wtmn4). This is done because real $* 8$ variable did not transfer in subroutine calling sequence properly, for no reason that was ever discovered. Line 736.

b. Further process blank history information to create 6-sample blank averaged activity and 20-sample blank standard deviation-call hblkinf(debug,xnumb,xnucl,wtmn'4,actmult,dpm_alarm,cer_nucl). Line 738.

H. Check to see if y12_bioassay_output.eq. 'YES'.or. new_reagent_calc(1:1) .eq. ' $Y^{\prime}$ '. Lines 764, 798.

1. If so, calculate MDA and $L_{c}$.

a. Return blank standard deviation-call gtsbblk(xnumb,sbblnk,debug). Convert result to dpm. Lines 766 through 768.

b. Return background standard deviation-call gtsbbkg(xnumb,bkgtim,sbblnk, debug). Line 770 .

c. Set $L_{c}=-1$ as a flag. If the value of $L_{c}$ is not set $\geq 0$ in later steps, that is a flag that no usable value of $L_{c}$ has been calculated. Line 775 .

d. Check to see that usable values were returned for both standard deviations ( $\operatorname{sbblnk}>-10^{25}$ and. sbbkgd $>-10^{25}$ ). Lines 776,797 .

(1) If so (i.e. both standard deviations $>-10^{25}$ ), calculate $M D A$ and $L_{c}$.

(a) Convert background standard deviation in counts/time to counts. Line 778. 
(b) Convert background standard deviation in counts to activity. Lines 781,782 .

(c) Sum the squares of the standard deviations. Line 785.

(d) Calculate MDA and $\mathrm{L}_{\mathrm{c}}$ in activity units. Lines 787 through 790 .

I. Check to see if y12_bioassay_output .eq. 'YES'.or. new_reagent_calc(1:1) .eq. ' $Y$ '. Lines 808, 815.

1. If so,

a. Put $\mathrm{L}_{\mathrm{c}}$ into variable $M P C$ in place of variable $C R I T$ provided that a usable value of $\mathrm{L}_{\mathrm{c}}$ has been calculated $(l s u b c \geq 0)$. Lines 810 through 814 .

J. Calculate modified sample error calculation for sample activity (wtmean) $=0$. This branch selected by if (wtmean .eq. 0) go to 9910 statement at line 823 and 9910 continue at line 892 in unmodified code. This block is avoided for wtmean $\neq 0$ by go to 9911 at line 888 , which skips to 9911 continue at line 909, which is below the modified sample error calculation. Lines 768 and 775 through 786.

1. Check to see if $y 12$ bioassay_output .eq. 'YES' .or. new_reagent_calc(1:1).eq. ' $Y$ '. Lines 897 , 908.

a. If so,

(1) Sample error (wtmaer) approximated by sqrt(2)* blank standard deviation. Also, result converted from blank standard deviation units, which were changed from $\mu \mathrm{Ci}$ to $\mathrm{dpm}$ in line 768 , back to $\mu \mathrm{Ci}$. Lines 904 through 906 .

K. Messages written for printing after execution of $A L P H A \_M D A$ giving information for each nuclide including MDA and $\mathrm{L}_{\mathrm{c}}$, if appropriate. Lines 919 through 935.

1. Check to see if (y12_bioassay_output .eq. 'YES'.or. new_reagent_calc(1:1) .eq. ' $Y$ ') .and. lsubc .lt.0.. Lines $919,920,924,935$.

a. If so,

(1) Write line of data including $x$ rncl,,,MDA. Lines 921, 922.

b. else,

(1) Write line of data including $x n u c l,, M D A, \underline{m p c}$ (note addition of $m p c$ to output list). Lines $925,926$.

(2) If $x n u c l$.ne. CER_nucl .and. rbblnk .gt. -1.e25 (i.e., if this is not the certificate nuclide and there is a usable value for $r b b \ln k$ ), then write additional line of data giving $r b b \operatorname{lnk}$ in dpm. Lines 930 through 933.

L. Rearrangement of statement 9900 and cam_pnucl statement with addition of checks to verify that blank and background files are okay. Lines 961 through 975.

1. Statement 9900 changed to continue. Line 961.

2. Check to see if $y 12$ bioassay_output .eq. 'YES'.or. new_reagent_calc(1:1).eq. ' $Y$ '. Lines 970 , 973.

a. If so,

(1) Check blank history file to see if it is okay (gives opportunity to stop operation of job)_call hblkchk(debug). Line 971.

(2) Check background history file to see if it is okay (gives opportunity to stop operation of job)—call hbkgchk(debug,bkgbat_id,bkgident). Line 972.

3. - Call $s=c a m \_$pnucl(c,etc.) separated from statement 9900 . Line 975.

M. Check to see if y12_bioassay_output.eq. 'YES'.or. new_reagent_calc(1:1) .eq. 'Y'. Lines $985,998$.

1. If so,

a. Write updated information on blank history file-call hblkwri(debug,nbr). Line 990.

b. Write updated information on background history file-call hbkgwri(debug,nbr,bkgbat_id,bkgident). Line 996.

N. Yield maximum of $125 \%$ changed to $115 \%$ to be consistent with Y12.FOR (125 may be a typographical error). Lines $1021,1022$. 


\section{SUBROUTINES FOR REAGENT BLANK AND BACKGROUND HISTORY INFORMATION AND OTHER AUXILIARY OPERATIONS}

A. Reagent blank information-subroutine hblank.

A.1. Initialization of blank information—entry hblkini(debug,nbr,batch_id,sident,yield,time_str). Lines

1137 through 1469 of subroutine hblank.

a. Initialize flags for file already checked, duplicate sample, and warning counters. Lines 1145 through 1150.

b. Get character strings representing matrix type and library to construct blank history file name. Lines $1152,1155$.

c. Get file type and therefore sample type. Lines 1158,1159 . The reason for this is to determine whether the sample is a reagent blank (xchar .eq. ' $R$ ).

d. Check to see if the length of the file type and sample type variables is small enough to construct a usable file name. Line 1169, 1441, 1467.

(1) If so,

(a) Construct blank history file name. History file name is “dub1:[alpha.alusr.histry.blank]matrix_controllib_blank.his." Lines 1170 through 1178.

(b) Construct blank archive file name. Archive file name is "dub1:[alpha.alusr.histry.blank]matrix_controllib_blank.ark." Lines 1180 through 1183.

(c) Set blank history and archive unit numbers and open blank history and archive files. Archive file is opened with 'append' access so that information will be written at end of file. Lines 1184 through 1194.

(d) Check to see if the sample is not a reagent blank (xchar .ne. ' $R$ ). Lines 1197, 1246, 1439.

i) If so (i.e., sample is not a reagent blank), the 6-sample averaged activity and 20-sample standard deviation calculated from the most recent reagent blank will be used to correct the sample activity for the blank and to calculate MDA and $L_{c}$. Information from the most recent reagent blank sample is stored in the first information set in the blank history file.

The structure of the blank history file is as follows:

Record 1: character variables [format $(1 \mathrm{x}, \mathrm{a} 16,1 \mathrm{x}, \mathrm{a} 16,1 \mathrm{x}, \mathrm{a} 20)$ ] giving batch ID and sample ID of most recent blank sample, plus date and time sample added to history file.

Records 2 through nbr+1: each record is 3 real $^{* 4} 4$ variables [format (1x,3g15.8)] giving the activity, 6-sample averaged activity, and 20-sample standard deviation for the most recent blank for each nuclide in the sample. Records nbr +2 through $2 *(n b r+1)$ : sample $\mathrm{ID}$, plus activity, 6-sample averaged activity, and 20 -sample standard deviation for the sample before the most recent blank for each nuclide in the sample.

Records $2 *(n b r+1)+1$ through $20 *(n b r+1)$ : additional samples in order of ever-increasing age. Note that the code is set up to count the number of information sets read and stop reading when it encounters an end of file (EOF). Thus, as much information as is available is read. If there are not 20 information sets on the history file, then the calculations will be done with those sets that are available. 
a) Read the batch and sample IDs, plus date and time sample added to history file. Lines 1198 through 1203. An EOF goes to statement 991 [see par. II.A.1.d.(1)(d)i)b)].

b) Read the activity, 6-sample averaged activity, and 20-sample standard deviation for the most recent blank. As has already been said, there will be one record of this information for each nuclide. Lines 1211 through 1223. An EOF goes to statement 991 [see par. II.A.1.d.(1)(d)i)b)].

1)) An EOF during the history information read goes to statement 991, which is described here. If an EOF is encountered, set the first blank sample ID and batch ID to EOF indicator fields, set date and time to current date and time, and set the counter for information read (nisnum) to 0 . Next, write an EOF notice message to TTY. Last, rewind the blank unit and send a notice message to TTY that information in blank history file will be new. Lines 1226 through 1241.

c) Initially set flag indicating blank sample is okay (lblsok) to true. Line 1245.

ii) Else, if the sample is a reagent blank, the previous up to 20 sets of reagent blank information must be read from the history file. Later, in entry hblkinf, a new set of averages and standard deviations will be calculated using the present blank information and that from previous blanks (see par. II.A.2.a.(5)). The information from the present blank sample will be stored in the first information set. Those from previous samples will be stored in memory locations for information sets 2 through 21 .

a) Initialize the running sums for calculating 6-sample averaged activity and 20-sample standard deviation. Lines 1252 through 1258.

b) Set the first blank sample ID and batch ID to values for current sample, set date and time to current date and time, and set the counter for information read (nisnum) to 1. Set flag indicating blank sample is okay (lblsok) to true. Set counter saying what memory location to insert values read from history file $(n 1)$ to 2 . Lines 1260 through 1265.

c) Check the state of the sample. This set of statements is copied from Y12.FOR with some modifications. Its mission is to find out what is the state of the sample. If the state is anything but "ok," the sample will not be added to the history file. The logic for checking the state for a reagent blank was reproduced in ALPHA_MDA.FOR rather than having the determination of the state left to Y12.FOR because ALPHA_MDA.FOR needs to know whether a reagent blank sample is not "ok" so it can refuse to add a flawed sample to the history file. The state is passed to a disk file to be read by Y12.FOR so that the latter code will not have to ask the operator to give the state of the sample a second time. Later, in hblkinf, tests are performed to see if the activity of the sample exceeds the dpm alarm limit [see par. II.A.2.a.(1)(c)]. 
1)) Pass message to TTY indicating that ALPHA_MDA is processing blank sample and giving sample and batch $\mathrm{Ds}$. Lines 1267 through 1269.

2)) See if yield is out of range. If so, set state to "unsuport" and write messages to TTY indicating that yield is out of range and that operator will have a chance to say why. Lines 1271 through 1279.

3)) Ask operator what is state of sample. Lines 1280 through 1299.

4)) Set state to values other than "ok" if operator has given such a response. Lines 1301 through 1307.

5)) Check to see if operator said state is "ok" and yield is within range. Lines 1318, 1319, 1323, 1328.

a) If so, set state to "ok." Also, set flag indicating sample is okay (lblsok) to true. Lines 1320 through 1322.

b)) Else, set the counter for information read (nisnum) to 0, set counter saying what memory location to insert values read from history file $(n l)$ to 1 , and set flag indicating sample is okay (lblsok) to false. Lines 1325 through 1327.

6)) Open disk file to pass sample state to Y12.FOR (file is "dub1:[alpha.alusr.histryljxktemp.da"), write state to file, and close file. Lines 1333 through 1348.

d) Read the batch $\mathrm{ID}$, sample $\mathrm{ID}$, date and time sample was added to history file, activity, 6-sample averaged activity, and 20-sample standard deviation for all the previous blank samples. Increment the counter for information read (nisnum) as each sample is read. Lines 1350 through 1378, 1400, and 1460.

1)) An EOF encountered while reading the activity, 6-sample averaged activity, and 20 -sample standard deviation goes to statement 1001. After statement 1001, decrement the counter for information sets read (nisnum) and go to statement 1009 (line 1418), which is the rest of the end of file logic. Lines 1374 through 1377.

e) Increment the sums for 6-sample averaged activity and 20-sample standard deviation. Lines 1387 through 1399.

f) Check to see if batch ID and sample ID of sample just read from history file match those of first sample, sample being read is not the first, and that no sample matches have so far been found [nisnum.gt.] .and. blkid(nisnum).eq.blkid(1).and. blkbid(nisnum).eq.blkbid(1) and. .not.lbldup]. Lines 1403 through 1405, 1415.

If so,

1)) Set flag that indicates duplicate blank sample (lbldup) to true. Line 1406.

g) An EOF reading the batch and sample IDs goes to statement 1009, whose operations are described here. Also, this statement is executed if the code successfully reads all 20 sets of history information. Line 1418.

1)) If nisnum $<1$, write a message to TTY saying that "blank history end of file reached before any info sets read," set the counter for 
information sets read (nisnum) to zero. Lines 1421 through 1425.

2)) If current batch and sample IDs duplicate any others (i.e., lbldup is true), put all information (i.e., batch $\mathrm{ID}$, sample ID, date and time sample entered into history file, activity, 6-sample averaged activity and 20-sample standard deviation for all nuclides) from memory location 2 into memory location 1 . No calculations of 6-sample averaged activity and 20 -sample standard deviation will be done. Instead, information from the most recent sample read off the history file will be used as if the current sample did not exist. Lines 1429 through 1438.

h) Set the flag for a usable blank history file name (lflblk) to true. Line 1222.

e. Else, blank history file name will be too long. Line 1441 .

then

(1) Write a message to TTY stating that no blank history file can be opened. Lines 1444 through 1446.

(2) Set flag for a usable blank history file name (lflblk) to false. Line 1417.

(3) Send a message to TTY stating that usable history file cannot be created and asking if ALPHA_MDA should be terminated immediately. Lines 1449 through 1463.

(a) If so, exit with call to SFTEXI. Lines 1464 through 1466.

A.2. Further processing of blank history information to create 6-sample averaged activity and 20-sample standard deviation--entry hblkinf(debug,xnumb,xnucl,wtmn4,actmult,dpm_alarm,cer_nucl). Lines 1472 through 1640 of subroutine hblank.

a. Check to see if this is a reagent blank sample, that the sample is okay, and the sample does not duplicate one on the history file (xchar.eq ' $R^{\prime}$.and. lblsok .and. .not.lbldup). Line 1482, 1639.

(1) If so,

(a) If this is first nuclide ( $x$ numb .eq. 1), set sum of sample activities and number of samples exceeded dpm alarm counter (nsmpex) to zero. Lines 1487 through 1490.

(b) Add activity for this nuclide to sum of sample activities provided that this is not the tracer isotope (xnucl .ne. cer_nucl). Line 1492.

(c) Test to see if the sum of sample activities and the activity for this nuclide both exceed the dpm alarm limit. Lines 1493 through 1494, 1558.

i) If so,

a) Set sample is okay flag (lblsok) to false. Line 1496.

b) Write warning messages to TTY stating that sample has exceeded dpm alarm limit and wait for acknowledgement. Lines 1498 through 1533.

c) Increment number of samples exceeded dpm alarm counter (nsmpex). Line 1534.

d) If counter is greater than 1, write messages to TTY pointing out that this is not the first nuclide to exceed dpm alarm limit. Lines 1535 through 1545.

e) Send a message to TTY asking if ALPHA_MDA should be terminated immediately. Lines 1549 through 1554.

1)) If so, exit with call to SFTEXI. Lines 1555 through 1557. 
(2) Check to see if sample okay flag (lblsok) flag has been changed to false. Lines 1561 , 1568.

(a) If so, return to main routine. Line 1567.

(3) Load the activity into the memory location for the most recent sample. Line 1570.

(4) Finish the sums for the six-sample averaged activity and standard deviation. The samples-added counters (ism $x 6$ and ism $x 20$ ) are incremented only for nuclide 1 to avoid counting more than once per sample. Lines 1571 through 1579.

(5) Calculate the six-sample averaged activity and standard deviation. Lines 1581 through 1636.

(a) Check to see if there is at least one usable sample for the six-sample averaged activity (ismx6 >0). Lines 1581, 1588, 1591 .

i) If so, calculate six-sample averaged activity. Line 1582.

ii) Else, set six-sample averaged activity to zero and write a message to that effect to TTY. Lines 1589, 1590.

(b) Check to see if there are at least four usable samples for the 20-sample standard deviation (ismx20 .ge. nsmpmn [=4]). Line 1594, 1623, 1636.

i) If so,

a) Calculate 20-sample standard deviation. Lines 1595, 1596, 1618.

b) If result of truncation errors in sum will cause standard deviation to be imaginary, set standard deviation to zero, send a message to TTY saying that standard deviation was not going to be real, and wait for acknowledgement of notice. Lines 1603 through 1616.

ii) Else,

a) Set 20-sample standard deviation to zero, send notice message to TTY, and wait for acknowledgement. Lines 1624 through 1635.

(c) If there were no blank history data lines read, but this sample is okay, set the counter for information sets read (nisnum) to one. Line 1638.

A.3. Return blank standard deviation-entry gtsbblk(numb,sbblnk,debug). Lines 1643 through 1677 of subroutine hblank.

a. Set standard deviation (sbblnk) to most recently calculated value. Line 1646 .

b. Check to see if there are no usable blank history data available (nisnum $<1$.or. .not.lflblk). Lines 1653, 1674.

(1) If so, (i.e., no usable data available),

(a) Write notice messages to TTY and wait for response (provided that this is the first time such a notice has been needed for this sample). Lines 1654 through 1671 .

(b) Increment the warning counter. Line 1672.

(c) Set sbblnk equal to -1.1e25 as a flag. Line 1673.

A.4. Return blank averaged activity-entry gtrbblk(numb,sbblnk,debug). Lines 1680 through 1718 of subroutine hblank.

a. Set averaged activity ( $r b b \operatorname{lnk})$ to most recently calculated value. Line 1683 .

b. Check to see if this is a blank reagent sample (xchar .eq.' $R$ ). Lines $1686,1688,1715$.

(1) If so, set rbblnk equal to $-1.1 \mathrm{e} 25$, which is a flag to the main routine not to correct for blank averaged activity. Line 1687.

(2) Else, check to see if there are no usable blank history data available (nisnum $<1$.or. .not.lflblk). If so, (i.e., no usable data available), send notice messages to TTY and wait for acknowledgement of notice. Then, set rbblnk equal to -1.1e25. Lines 1693 through 1714. 
A.5. Check to see if it is okay to write updated information on blank history file-entry hblkchk(debug). Lines 1721 through 1759 of subroutine hblank.

a. Check to see if this file has already been checked (lflchk is true). Lines $1723,1726,1728$.

(1) If so, return to main routine. Line 1725.

(2) Else, set file checked flag (lflchk) to true. Line 1727.

b. Check to see whether blank history file cannot be opened (.not.lflblk is true). Lines 1730 , $1732,1758$.

(1) If not (i.e., .not.lflblk is true), then return to main routine. Line 1731.

(2) Else, check to see if this is a blank reagent sample, that the sample is okay, and that the sample does not duplicate one already in the history file ( $x$ char .eq. ' $R$ ' .and. lblsok .and .not.lbldup). Lines 1737, 1757.

(a) If so, check to see if no usable blank history data exist (nisnum $<I$ ). Lines 1739 , 1756.

i) If so, (i.e., no usable blank history data available),

a) Write a message to TTY stating that no usable blank history data are available and that empty history file will be written. Lines 1741 through 1745.

b) Send a message to TTY asking if ALPHA_MDA should be terminated immediately and wait for response. Lines 1746 through 1752 .

1)) If so, exit with call to SFTEXI. Lines 1753 through 1755.

A.6. Write updated information on blank history file-entry hblkwri(debug,nbr). Lines 1762 through 1814 of subroutine hblank.

a. Check to see if the blank history file name is not okay and that a history file cannot be opened (.not.lflblk). Lines 1764, 1768, 1813.

(1) If so (i.e., blank history file is not okay), write a message to TTY to that effect and go to end if at line 1813. Lines 1765 through 1767.

(2) Else, check to see if this is a blank reagent sample, that the sample is okay, and that the sample does not duplicate one already in the history file (xchar .eq.' $R{ }^{\prime}$.and. lblsok and .not.lbldup). Lines $1773,1812$.

(a) If so, check to see if no usable blank history data exist (nisnum $<1$ ). Lines 1775, 1781 .

i) If so, (i.e., no usable blank history data available), write a message to that effect to TTY, write an empty file to blank history, and return to main routine. Lines 1776 through 1780.

ii) Else, write up to 20 sets of blank history data-batch $\mathrm{ID}$, sample $\mathrm{ID}$, and date sample added to history file; plus activity, 6-sample averaged activity, and 20-sample standard deviation for each nuclide. First line in each set has characters 31 through 57 of the blank history file name written at the end. Lines 1782 through 1811.

B. Background information-subroutine hbkgnd.

B.1. Initialization of background information-entry hbkgini(debug,nbr,bkgident). Lines 1863 through 2135 of subroutine hbkgnd.

a. Initialize flags for file already checked, duplicate sample, and warning counters. Lines 1873 through 1877.

b. Get character strings representing chamber and library to construct background history file name. Construct chamber number string to have three characters with leading zeroes (e.g., chamber 1 has chamber='001'). Lines 1879 through 1892.

c. Check to see if the length of the file type and sample type variables is small enough to 
construct a usable file name. If not, skip down to else statement at line 1848 (see par. II.B.1.d). Lines 1901, 2133.

(1) Construct background history file name. History file name is “dub1:[alpha.alusr.bkgd]chamber_controllib_bkgnd.his." Lines 1902 through 1908.

(2) Define background unit number and open background history file. Lines 1909 through 1917.

(3) Read batch and sample IDs, live time, and date and time sample added to history file for first background sample on history file. An EOF goes to statement 981 at line 1931 [see par. II.B.1.c.(3)(a)]. Lines 1919 through 1923.

(a) An EOF during the read of the first sample ID from the history information goes to statement 981 , which is described here.

i) Set the first background batch ID (bkgbidl), sample ID (bkgidI), first line batch ID [bkgbid(1)], and background sample ID [bkgid(1)] to EOF indicator fields. Set both the counter for information read (nisnum) and the live time for line $1[\operatorname{ltimbk}(1)]$ to zero. Set the date and time sample added into history field [bkgpdat(1)] to current date and time (time_str). Lines 1932 through 1938.

ii) Write a notice message to TTY saying that EOF was reached on background history file before first record finished reading and that there is no background history information. Lines 1941 through 1953.

iii) Rewind background history file so that either it will be empty or else that information from the current background will be first on the history file and write a message to TTY explaining that new information will go into history file. Lines 1956 through 1960.

(4) Check to see if batch ID and ID of first sample on history file are same as current background batch ID and background sample ID (bkgidl.eq.bkgident and. bkgbidl.eq.bkgbat_id). Lines 1965, 2005, 2108.

(a) If so (i.e., IDs match),

i) Read the 20-sample standard deviation calculated from the most recent background. This will be used to calculate MDA and $\mathrm{L}_{\mathrm{c}}$. Information from the most recent background sample is stored in the first information set in the background history file.

The structure of the background history file is as follows:

Record 1: character variables [format $(1 \mathrm{x}, \mathrm{a} 24,1 \mathrm{x}, \mathrm{a} 16, \mathrm{~g} 15.8, \mathrm{a} 20)$ ] giving batch and sample IDs of most recent background sample, Real*4 variable giving live time for this sample, plus character variable giving date and time sample added to history file.

Records 2 through nbr+1: each record is two real*4 variables [format (1x,2g15.8)] giving the background counts/time and 20-sample standard deviation for the most recent background for each nuclide in the sample.

Records nbr +2 through $20 *(n b r+1)$ : additional samples in order of everincreasing age. Note that the code is set up to count the number of information sets read and stop reading when it encounters an EOF. Thus, as much information as is available is read. If there are not 20 information sets on the history file, then the calculations will be done with those sets that are available. 
a) Set the counter for information read (nisnum) to 1 and set the first line batch and background IDs [bkgbid(1) and bkgid(1)] to the first line values already read (bkgbidl and bkgidl). Lines 1966 through 1968.

b) Read the background counts/time and 20-sample standard deviation for the most recent background. As has already been said, there will be one record of this information for each nuclide. Lines 1969 through 1978. An EOF goes to statement 991 [see par. II.B.1.c.(4)(a)i)b)].

1)) An EOF during the history information read goes to statement 991, which is described here. Set the first background batch and sample IDs (bkgbidl and bkgidl) and the first line background batch and sample IDs [bkgbid(1) and bkgid(1)] to EOF indicator fields. Set both the counter for information read (nisnum) and the live time for line 1 [ltimbk(1)] to zero. Set the date and time sample added to history file [bkgpdat(1)] to current date and time. Next, write an EOF notice message to TTY. Last, rewind the background unit and send a notice message to TTY that information in background history will be new. Lines 1982 through 2003.

(b) Else (background batch and sample IDs do not match the most recent background history batch and sample IDs), the previous up to 20 sets of background history information must be read from the history file. Later, a new set of standard deviations must be calculated using the present background information and that from previous backgrounds [see par. II.B.2.a.(1)(c)]. The information from the present background sample will be stored in the first information set. Those from previous samples will be stored in memory locations for information sets 2 through 21. Line 2005.

i) Initialize the running sums for calculating 20-sample standard deviation. Lines 2012 through 2016.

ii) Set the first batch and sample IDs to values for current sample. Set first live time to zero (correct live time will be placed in this location by entry hbkginf). Set first date and time sample added to history file to current date and time. Set counter for information read (nisnum) to 1. Rewind background unit to begin reading again. Lines 2018 through 2025.

iii) Read the batch and sample IDs, live time, and date and time sample added to history file; plus background counts/time and 20-sample standard deviation for all the previous background samples. Increment the counter for information read (nisnum) as each sample is read. Lines 2026 through 2051, 2068 , and 2084.

a) An EOF encountered while reading the activity and 20-sample standard deviation goes to statement 1001 at line 2052. At statement 1001 , decrement the counter for information sets read (nisnum) and go to statement 1009 at line 2086, which is the rest of the EOF logic. Lines 2052 through 2055.

iv) Increment the sums for 20 -sample standard deviation. Lines 2063 through 2067.

v) Compare batch and sample IDs of sample just read from history file with those of first sample. This is to be done provided sample being read is not the first and that no sample matches have so far been found. If patterns 
match, set flag that indicates duplicate background sample (lbkdup) to true. Lines 2071 through 2080.

vi) An EOF reading the sample ID goes to statement 1009, whose operations are described here. Also, this statement is executed if the code successfully reads all 20 set of history information. If the counter for information sets read (nisnum) is $<1$, this flags that there were no complete information sets read. Line 2086.

a) If nisnum < 1, write a message to TTY saying that "background history end of file reached before any info sets read," set the counter for information sets read (nisnum) to zero. Lines 2090 through 2095.

b) If current batch and sample IDs duplicate any others (i.e., lbkdup is true), put all information (i.e., batch ID, sample ID, live time, date and time sample entered into history file, activity, and 20-sample standard deviation for all nuclides) from memory location 2 into memory location 1. No calculations of 20-sample standard deviation will be done. Instead, information from the most recent sample read off the history file will be used as if the current sample did not exist. Lines 2098 through 2107.

vii) Set the flag for a usable background history file name (lflbkg) to true. Line 2109.

d. Else (background history file name will be too long), Line 2110.

(1) Write a message to TTY stating that no background history file can be opened. Lines 2113 through 2115 .

(2) Set flag for a usable background history file name (lflbkg) to false. Line 2116.

(3) Send a message to TTY asking if ALPHA_MDA should be terminated immediately. Lines 2118 through 2129.

(a) If so, exit with call to SFTEXI. Lines 2130 through 2132.

B.2. Further processing of background history information to create 20-sample standard deviation-entry hbkginf(debug,numb,xnucl,bkgcts,bkgtim,bkgbat_id,bkgident). Lines 2138 through 2222 of subroutine hbkgnd.

a. Check to see if batch and sample IDs of first sample on history file are not the same as current background batch and sample IDs and that the sample does not duplicate one on the history file ((bkgid1.ne.bkgident .or. bkgbidl.ne.bkgbat_id) .and. .not.lbkdup). Lines 2151, 2152, 2221.

(1) If the batch and sample IDs do not match and sample is not a duplicate, then

(a) Load the background counts/time and live time into the memory location for the most recent sample. Lines 2155, 2156.

(b) Finish the sums for the standard deviation. The samples added counter (ismx20) is incremented only for nuclide 1 to avoid counting more than once per sample. Lines 2157 through 2163.

(c) Calculate the standard deviation. Lines 2168 through 2197.

i) Check to see if there are at least 4 usable samples for the 20-sample standard deviation (ismx20 > nsmpmn [=4]). Lines 2168, 2197, 2218.

a) If so,

1)) Calculate the 20 -sample standard deviation. Lines 2169,2170 , 2192.

2)) If result of truncation errors in sum will cause standard deviation to be imaginary, set standard deviation to zero, send a message 
to TTY saying that standard deviation was not going to be real, and wait for acknowledgement of notice. Lines 2177 through 2190.

b) Else, the standard deviation is not good enough, so none will be calculated. Line 2197.

1)) Save the negative of the square root of the background counts. The minus sign is a flag that the value stored is not a real standard deviation, but is rather this square root. Line 2203 .

2)) Write message to TTY stating that square root of background counts replaces standard deviation and that number of samples < nsmpmn (=4). Wait for acknowledgement. Lines 2204 through 2216.

ii) If there were no background history data lines read (i.e., nisnum.lt.l), set the counter for information sets read (nisnum) to one. If the code gets to this point, this sample should be okay. Line 2220 .

(2) If the background batch and sample IDs match or sample is a duplicate, then no further calculations will be done-skip to the end if at line 2221 and return to the main routine.

B.3. Return background standard deviation-entry gtsbbkg(numb,bkgtim,sbblnk,debug). Lines 2225 through 2273 of subroutine hbkgnd.

a. Set standard deviation (sbblnk) to most recently calculated value. Line 2228 .

b. Check to see if the standard deviation stored in memory is negative. If so, the value stored is minus the root of the number of counts [see par. II.B.2.a.(1)(c)i)b)]. Set sbblnk equal to the root of the counts divided by the live time. Lines 2232 through 2247.

c. Check to see if there are no usable background history data available (nisnum $<1$.or. .not.lflbkg). Lines $2250,2270$.

(1) If so, (i.e., no usable data available),

(a) Write notice messages to TTY and wait for response (provided that this is the first time such a notice has been needed for this sample). Lines 1975 through 2267.

(b) Increment the warning counter. Line 2268.

(c) Set sbblnk equal to $-1.1 \mathrm{e} 25$ as a flag. Line 2269 .

B.4. Check to see if it is okay to write updated information on background history file-entry hbkgchk(debug,bkgbat_id,bkgident). Lines 2276 through 2323 of subroutine hbkgnd.

a. Check to see if this file has already been checked (lflchk). Lines 2285, 2288, 2290.

(1) If so, return to main routine. Line 2287.

(2) Else, set file checked flag (lflchk) to true. Line 2289.

b. Check to see whether background history file cannot be opened (.not.lflbkg is true). Lines 2292, 2294, 2322.

(1) If not (i.e., .not.lflbkg is true), then return to main routine. Line 2293.

(2) Else, check to see if batch and sample IDs of first sample on history file are not the same as current background batch and sample IDs and that the sample does not duplicate one on the history file ((bkgidl.ne.bkgident .or. bkgbidl.ne.bkgbat_id) .and. .not.lbkdup). Lines 2300, 2301, 2321.

(a) If the batch and sample IDs do not match and sample is not a duplicate, then

i) Write a message to TTY stating that no usable background history data are available and that empty history file will be written. Lines 2305 through 2309. 


\section{2-14}

ii) Send a message to TTY asking if ALPHA_MDA should be terminated immediately and wait for response. Lines 2310 through 2316.

\section{B.5. Write updated information on background history file-entry} hbkgwri(debug,nbr,bkgbat_id,bkgident). Lines 2326 through 2382 of subroutine hbkgnd.

a. Check to see if the background history file name is not okay and that a history file cannot be opened (.not.lflbkg). Lines 2335, 2339, 2381.

(1) If so (i.e., background history file is not okay), write a message to that effect to TTY and go to end if at line 2381. Lines 2336 through 2338.

(2) Else, check to see if batch and sample IDs of first sample on history file are not the same as current background batch and sample Ds and that the sample does not duplicate one on the history file [(bkgidl.ne.bkgident.or. bkgbidl.ne.bkgbat_id) .and. .not.lbkdup]. Lines 2345, 2346, 2380.

(a) If so, check to see if no usable background history data exist (nisnum $<1$ ). Lines $2348,2354$.

i) If so, (i.e., no usable background history data available), write a message to TTY to that effect, write an empty file to background history, and return to main routine. Lines 2349 through 2353.

ii) Else, write up to 20 sets of background history data-batch and sample IDs, live time, and date and time sample added to history file; plus activity and 20-sample standard deviation for each nuclide. First line in each set has characters 31 through 52 of the blank history file name written at the end. Lines 2355 through 2379.

C. Pack real number in coded form into integer-subroutine reinpak(relnum, intnum). Lines 2387 through 2434. Given a real*4 number (relnum), this routine creates a 1 pe13.6 format in a particular coded fashion that is returned as an integer (intnum). If the number in the 1pe13.6 format is i.jklmnoe+ab, then the routine returns an integer abijklmno. If the number in the 1pe13.6 format is $i . j k l m n o e-a b$, then the routine returns an integer - abijklmno. The number itself (i.jklmno) is placed in the seven rightmost digits with the decimal point suppressed and the exponent is placed in the eighth and higher digits from the right.

D. Unpack number in integer coded form into real-subroutine inreupk(relnum, intnum). Lines 2437 through 2471. The integer coded format is explained in the discussion of the packing routine reinpak (see par. II.D). Subroutine inreupk reverses the process described for subroutine reinpak and returns the real*4 number (relnum) that is represented by the coded integer number (intnum).

E. Exit and ask whether PROCESS.COM should also be terminated (soft exit)-subroutine sftexi(iflexi). Lines 2474 through 2503.

1. Send a message to TTY saying that ALPHA_MDA is exiting and asking if PROCESS.COM should also terminate. Wait for a response. Lines 2479 through 2489.

2. If response is not ' $n$ ' or ' $N$ ', send message to TTY saying that operator has told PROCESS.COM to quit. Lines 2492, 2493.

3. Else,

a. Send message to TTY saying that operator has told PROCESS.COM to continue. Line 2495.

b. Change response message variable (chyno) to ' $\mathrm{g}$ '. Line 2498.

4. Send response message variable (chyno) to system flag variable alphaerr. Line 2501 .

5. Call exit. Line 2502.

F. Ring bell on TTY - subroutine belrng(nring). Ring bell nring times pausing about $1 \mathrm{~s}$ between each ring. Note that while this is called a bell, the normal sound on a TTY is a beep. Lines 2506 through 2515. 


\subsection{NDAL_PROCESS.COM}

In NDAL_PROCESS.COM, there are a few changes near the call to ALPHA_MDA. There are other changes in the file that make it possible to process the background samples independently of the reagent blank and regular samples. A number of lines were added so that the warning and notice messages from processing the background samples independently could be written to a temporary file and then printed. The reason for this is that, in ALPHA_MDA, a number of messages are written to TTY followed by a halt in the processing until the operator acknowledges the warning. Most of these messages come about because something is "wrong" with the history file being accessed. Usually, what is wrong is that the file is nonexistent or empty or simply does not have enough lines of history information to make a proper standard deviation. This would happen whenever a history file was deleted either accidently or by some system failure, or if the file was corrupted. The reason for issuing warning messages and stopping the processing is so the operator will be aware that something has gone wrong with the history file and take steps to correct the problem. However, there are instances for which a history file may be deliberately deleted. That should happen whenever a detector is replaced. The reason is that the background count levels based upon the old detector will be different from the new levels and thus the standard deviation will suddenly get large. The history files are missing or too small when the history calculations are first introduced to a new system. When a history file is being reconstructed, the operator must sit at the console reading and acknowledging notice messages. The customers decided that, because the reconstruction of the background history files for a particular detector does happen periodically, they would rather have the processing of backgrounds through NDAL_BKGD_HISTRY.COM and BKGD_HIST.FOR generate warning and notice messages on paper for the operators to read after the background samples had been processed rather than force the operator to sit at the console and watch for messages. This is the reason for the additions that make and print the temporary background processing warning and notice message file.

The remainder of this section explains the changes in some detail to make future maintenance of the code easier. A listing of the entire NDAL_PROCESS.COM file is included as Appendix 2.B. The line numbers shown in the appendix at the first of each line are referenced in the discussion of the code in this section. All additions and changes by Kirkpatrick are bracketed by comment cards starting with "jxk," which is Kirkpatrick's computer user ID.

A. JXK modifications of the NDAL_PROCESS.COM file to process backgrounds separately are from lines 503 through 507. Modifications for handling of the warning and notice message file from processing backgrounds separately are from lines 147 through 184, 433 through 467, 513 through 545, and 1734 through 1776. Each block of changes is discussed below in turn.

1. Prepare and initialize a formatted disk file called [alpha.alusr.histry]jxktempnotemsg.dat. This file is intended to collect all of the notice messages from all of the background samples processed by this execution of PROCESS.COM.

a. Determine whether $y 12$ bioassay .eqs. ' $Y$ ' or new_reagent_calc eqs. ' $Y$ '. Lines 150 through $152,183$.

(1) If so,

(a) Construct the file name. Lines 153 through 156.

(b) Delete all old copies of the file provided that any exist. Lines 159 through 163.

(c) Open a new copy of the file with "append" access. Lines 168 through 174.

(d) Write header lines to the file. Set symbol jxknotehdlncnt $=2$ to indicate that there are two lines of header messages. Lines 178 through 181.

(e) Close the file. Line 182. 
2. Prepare and initialize a second formatted disk file called [alpha.alusr.histry]jxktempbkmsg.dat. This file is intended to collect all of the notice messages from a single background sample being processed at the present moment.

a. Determine whether $y 12$ bioassay .eqs. ' $Y$ ' or new_reagent_calc eqs. ' $Y$ '. Lines 435 through $437,466$.

(1) If so,

(a) Construct the file name. Lines 438 through 441.

(b) Delete all old copies of the file. Lines 444 through 447.

(c) Open a new copy of the file with 'append' access. Lines 452 through 459.

(d) Write header lines to the file. Set symbol jxkbkhdlncnt $=2$ to indicate that there are 2 lines of header messages. Lines 462 through 464.

(e) Close the file. Line 465 .

3. Process backgrounds separately using DCL file NDAL_BKGD_HISTRY.COM. Line 505.

4. Append results from file jxktempbkmsg.dat (from background sample just run) to file $j x k t e m p n o t e m s g . d a t$ provided that any notice messages have been written to file $j x k t e m p b k m s g . d a t$.

a. Determine whether $y 12$ bioassay.eqs. ' $Y$ ' or new_reagent_calc.eqs. ' $Y$ '. Lines 515 through $517,544$.

(1) If so,

(a) Set the lines read counter (jxklinecnt) to an initial value of 0 . Line 523.

(b) Open file jxktempbkmsg.dat and attempt to read a line from it three successive times. If an EOF is found, skip to label jxkbkeof at Line 533. Otherwise, increment the lines read counter (jxklinecnt) by one for each successful read. Lines 524 through 531 .

(c) Close file jxktempbkmsg.dat. Line 534.

(d) If number of lines read (jxklinecnt) $>$ number of header lines put into file in the first place (jxkbkhdlncnt), then append the contents of file jxktempbkmsg.dat to file jxktempnotemsg.dat. Lines 537 through 540.

5. Print results from file jxktempnotemsg.dat (from all background samples run in this execution of PROCESS.COM) to file $j x k t e m p n o t e m s g . d a t$ provided that any notice messages have been written to file.

a. Determine whether $y 12$ bioassay .eqs. ' $Y$ ' or new_reagent_calc .eqs. ' $Y$ '. Lines 1741 through $1743,1772$.

(1) If so,

(a) Set the lines read counter (jxklinecnt) to an initial value of zero. Line 1748.

(b) Open file jxktempnotemsg.dat and attempt to read a line from it three successive times. If an end of file is found, skip to label jxknoteeof at Line 1758. Otherwise, increment the lines read counter (jxklinecnt) by one 1 for each successful read. Lines 1749 through 1756.

(c) Close file jxktempnotemsg.dat. Line 1759.

(d) If number of lines read (jxklinecnt) $>$ number of header lines put into file in the first place (jxknotehdlncnt), print file jxktempnotemsg.dat. Lines 1762 through 1765.

(e) Delete all copies of file jxktempnotemsg.dat. Lines 1768 through 1771. 
B. So that the notice and error message file from processing backgrounds separately should be printed at the very end of a processing run, the "exit" statements in the PROCESS.COM file ahead of the subroutine for final quality assurance work (subroutine QA_PLOT_PARAM which starts at line 1778) were changed to "goto master_exit." Then, a "master_exit" statement was placed at line 1735, which is ahead of the QA_PLOT_PARAM subroutine). The changes from "exit" to "goto master_exit" occur at the following lines: 258, 1609, 1646, 1667, 1710, and 1730.

C. JXK modifications of the NDAL_PROCESS.COM file in the region around the execution of ALPHA_MDA are from lines 1285 through 1315.

1. Define SYS\$INPUT to be SYS\$COMMAND so that requests for a response from the ALPHA_MDA code will wait for a response from TTY rather than expecting to be passed something from the PROCESS.COM file. Line 1297.

2. Set the flag variable alphaerr to "n." Line 1298.

3. Check to see if the flag variable alphaerr is no longer " $n$ " after ALPHA_MDA. If alphaerr has been reset, this is a flag that ALPHA_MDA has terminated by command. Lines 1302, 1303, 1314.

a. If so, check to see if the flag variable alphaerr has been reset to " $g$ " by ALPHA_MDA. Lines 1306, 1307, 1310.

(1) If so, send a message to TTY saying that PROCESS.COM will go to next spectrum and go to label ask_next_spec. Lines 1308, 1309.

(2) Else, send a message to TTY saying that PROCESS.COM will abort as ordered and go to label abort_exit. Lines 1311 through 1313.

\subsection{Y12.FOR}

In Y12.FOR, there are a modest number of changes in the code. Kirkpatrick combined his changes with others done in January 1995 by J. L. Hilemon (computer user ID "jih"). The remainder of this section explains each of the changes in some detail to make future maintenance of the code easier. A listing of the entire Y12.FOR file is included as Appendix 2.C. The line numbers shown in the appendix at the first of each line are referenced in the discussion of the code in this section. All additions and changes by Kirkpatrick are bracketed by comment cards starting with "jxk", which is Kirkpatrick's computer user ID.

A. Read flag to determine whether this sample is a reagent blank (xchar). Lines 289, 290.

B. Preset the state read from disk (statrd) to 13 blanks. Line 293.

C. Check to see if this is a reagent blank sample (xchar.eq.' $R$ '). Lines $294,349$.

1. If so,

a. Set unit number for reading state from disk. Line 295 .

b. Open file dub1:[alpha.alusr.histry]jxktemp.dat, which contains state for a reagent blank passed from ALPHA_MDA. Lines 300, 301.

c. Read state from disk file. EOF goes to statement 181 at line 304. Line 302.

d. An EOF reading sample state goes to statement 181, whose operations are described here. Line 304.

(1) Send message to TTY saying that EOF was reached while reading state from disk. Line 307.

e. Close file from ALPHA_MDA with status='delete' to make sure file is deleted. Lines 310 , 311.

f. Check to see if the state read from disk (statrd) is any of eight values that represent a sample that is not okay: unsuport, lost 1, lost2, lost3, lost4, lost5, reproc, and recount. Lines 317 through 324.

(1) If any of those "if" statements is true, code takes the branch to statement 191 at line 340. 
(2) Else, check to see if dpm alarm exceeded detection loop in lines 235 through 282 of the unmodified code has determined that the dpm alarm was exceeded and passed a state of exceeded to this point. Line 328.

(a) If so,

i) Send a message to TTY saying that dpm alarm has been exceeded and that operator will be given a chance to explain why. Lines 329 through 332 .

ii) Take branch to statement 199 at line 350 . This branch goes to the unmodified code to ask for a change in state one of the states that represents a sample not okay. If the operator attempts to say that the sample is still okay, then the unmodified code will insist on retaining the exceeded state. Line 333.

g. Check to see if the state read from disk (statrd) is ok. Line 337.

(1) If so, code takes the branch to statement 191 at line 340.

(2) Else, take branch to statement 199 at line 350 . This branch goes to the unmodified code to ask for a state. This branch is taken if statrd contained no recognizable state. This branch usually happens when the file jxktemp.dat is empty or nonexistent. That is most likely to happen if somehow the ALPHA_MDA.FOR execution went bad or, more likely, if this job is not being run with the y12_bioassay_output flag set so that ALPHA_MDA.FOR never did any of the special operations that are the subject of the present report. Line 339.

h. If statrd contains a recognizable state and the dpm alarm was not exceeded, a go to 191 branch is taken. The operations at and after statement 191 are explained here. Line 340.

(1) Set state to value read from disk (statrd). Line 343.

(2) Send message to TTY saying that state already determined to be value passed and that no manual input of state is needed. Lines 344 through 347.

(3) Take branch to statement 299 at line 422 . This branch goes to the end of the modified code and resumes the unmodified code after the lines that accomplish the manual specification of the sample state. Line 349.

i. The branch to statement 199 goes to this point. The lines following this are unmodified code doing the manual setting of the sample state. Line 350.

j. The branch to statement 299 goes to this point. The lines following this are unmodified code after the manual setting of the sample state. Line 422 .

\subsection{BKGD_HIST.FOR CODE}

The initial operating versions of the software modifications (as of about May 1995) added a background to the history file whenever the background was used for a reagent blank sample or a normal sample. As a consequence, the results of many background measurements were never added to the history. In particular, backgrounds were not processed for nuclides that did not happen to have other samples processed during the week. The customers decided that it would be best to make further alterations to the software so that, when the background samples were initially processed, they would be added to the history file. That was not a difficult modification to make. The PROCESS.COM file already had a loop that processed all of the background samples (at least, all those whose names showed up in a NAMES.DAT file) using the regions and library for each of the nuclides passed in the variable "actinide_list." It was not difficult to construct a small DCL file that would be executed by NDAL_PROCESS.COM for each detector and each nuclide. The DCL file (NDAL_BKGD_HISTRY.COM) executes a FORTRAN file called BKGD_HIST. The BKGD_HIST.FOR file contains copies of the background history logic from ALPHA_MDA.FOR. In effect, 
it is a modified subset of ALPHA_MDA.FOR. A listing of the entire BKGD_HIST.FOR file is included as Appendix 2.D.

\section{OPERATIONS IN MAIN ROUTINE}

A. Get value of symbol bkgfilnam, which is the background file name complete with path as generated by NDAL_BKGD_HISTRY.COM. Convert to uppercase. Lines 103 through 105.

B. Open the background channel to alphaO. Lines 114 through 116.

C. Note: This code is not called unless y12_bioassay_output .eq. 'YES'. Therefore, it is not necessary to make an "if" statement to check to see if the rest of this code should be executed.

1. Find background batch $\mathrm{D}$ field ( $b$ kgbat_id) and truncate result to 24 rightmost characters. Lines 141 through 149.

2. Initialization of background history information-call - hbkgini(debug,nbr,bkgbat_id,bkgident,time_str). Line 152.

D. Note: This code is not called unless y12_bioassay_output .eq. 'YES'. Therefore, it is not necessary to make an "if" statement to check to see if the rest of this code should be executed.

1. Further process background history information to create 20-sample background standard deviation-call hbkginf(debug,xnumb,bkgcts,bkgtim,bkgbat_id,bkgident). Lines 173 through 182.

E. Note: This code is not called unless y12_bioassay_output .eq. 'YES'. Therefore, it is not necessary to make an "if" statement to check to see if the rest of this code should be executed.
a. Write
updated information
on background
history
file-call hbkgwri(debug,nbr,bkgbat_id,bkgident). Line 199.

\section{SUBROUTINES FOR REAGENT BLANK AND BACKGROUND HISTORY INFORMATION AND OTHER AUXILIARY OPERATIONS}

A. Note that this code does not deal with blank information, so no blank routines are present or discussed.

B. Background information-subroutine hbkgnd. This version of hbkgnd was copied from ALPHA_MDA.FOR. Some changes were made to reroute the warning messages to a temporary file rather than to TTY. Describing the operations of the hbkgnd subroutine of BKGD_HIST.FOR in detail would be redundant because the description would be virtually identical to that of ALPHA_MDA.FOR. Instead, only the differences between the two versions of hbkgnd are described below.

B.1. Initialization of background information-entry hbkgini(debug,nbr,bkgident). Lines 252 through 567 of subroutine hbkgnd.

a. Get symbol jxk_temp_bkmsg, which is name of the file to be used for writing warning messages. Open file on unit 13 with append access. Lines 254 through 257.

b. Check to see if debug(1:1) .eq. ' $Y$ '. Lines $381,398$.

(1) Only if this statement is true will the code send warning message to TTY and wait for response.

(2) Send duplicate set of warning messages to unit 13. Lines 399 through 420.

c. Check to see if debug(1:1) .eq. ' $Y$ '. Lines $470,473$.

(1) Only if this statement is true will the code send warning message to TTY and wait for response.

(2) Send duplicate set of warning messages to unit 13. Lines 474 through 477.

d. Check to see if debug( $1: 1)$.eq. ' $Y$ '. Lines 515,520 .

(1) Only if this statement is true will the code send warning message to TTY and wait for response.

(2) Send duplicate set of warning messages to unit 13. Lines 521 through 525.

B.2. Further processing of background history information to create 20-sample standard 
deviation-entry hbkginf(debug,numb,bkgcts,bkgtim,bkgbat_id,bkgident). Lines 570 through 688 of subroutine hbkgnd.

a. Check to see if debug(1:1).eq. ' $Y$ '. Lines 614,624 .

(1) Only if this statement is true will the code send warning message to TTY and wait for response.

(2) Send duplicate set of warning messages to unit 13. Lines 625 through 635 .

b. Check to see if debug(1:1).eq. ' $Y^{\prime}$. Lines 650,666 .

(1) Only if this statement is true will the code send warning message to TTY and wait for response.

(2) Send duplicate set of warning messages to unit 13. Lines 667 through 683.

B.3. Return background standard deviation-entry gtsbbkg(numb,sbblnk, debug). Lines 691 through 749 of subroutine hbkgnd. Note that bkgtim has been added to the argument list for entry gtsbbkg for ALPHA_MDA.FOR. Further note that gtsbbkg is not called from BKGD_HIST.FOR, so the changes described here are not relevant.

a. Check to see if $\operatorname{debug}(1: 1)$.eq. ' $Y$ '. Lines 704,723 .

(1) Only if this statement is true will the code send warning message to TTY and wait for response.

(2) Send duplicate set of warning messages to unit 13. Lines 724 through 743.

B.4. Check to see if it is okay to write updated information on background history file-entry hbkgchk(debug,bkgbat_id,bkgident). Lines 752 through 821 of subroutine hbkgnd. Note that hbkgchk is not called from BKGD_HIST.FOR, so the changes described here are not relevant.

a. Check to see if debug(1:1) .eq. ' $Y$ '. Lines 781, 798.

(1) Only if this statement is true will the code send warning message to TTY and wait for response.

(2) Send duplicate set of warning messages to unit 13. Lines 799 through 817.

B.5. Write updated information on background history file-entry hbkgwri(debug,nbr,bkgbat_id,bkgident). Lines 824 through 896 of subroutine hbkgnd.

a. Check to see if debug(1:1) .eq. ' $Y$ '. Lines $834,839$.

(1) Only if this statement is true will the code send waming message to TTY and wait for response.

(2) Send duplicate set of warning messages to unit 13. Lines 840 through 844 .

b. Check to see if debug(1:1) .eq. ' $Y$ '. Lines 855,859 .

(1) Only if this statement is true will the code send warning message to TTY and wait for response.

(2) Send duplicate set of warning messages to unit 13. Lines 860 through 864 .

\subsection{NDAL_BKGD_HISTRY.COM}

As was explained in the introduction to the BKGD_HIST.FOR file, the DCL file NDAL_BKGD_HISTRY.COM executes the FORTRAN file BKGD_HIST. The file NDAL_BKGD_HISTRY.COM is called by NDAL_PROCESS.COM from within a loop that processes all of the background samples in the NAMES.DAT file for each actinide in "actinide_list." A listing of the entire NDAL_BKGD_HISTRY.COM file is included as Appendix 2.E.

NDAL_BKGD_HISTRY.COM bears a strong resemblance to NDAL_PROCESS.COM, especially the portions of the latter that execute ALPHA_MDA to process the reagent blank and regular samples. NDAL_BKGD_HISTRY.COM is not so complex as to require a detailed explanation of its operations. 


\subsection{FORTOPEN.FOR}

FORTOPEN.FOR is a very short FORTRAN code intended to open a formatted file with FORTRAN and then close it with "keep" disposition. Its listing is Appendix 2.F. FORTOPEN.FOR was written because, when a file is opened by VMS and then written to by FORTRAN, the carriage return-line feed sequence put at the end of each FORTRAN line does not work properly. Instead, the entire file looks like one very long line. FORTOPEN.FOR is not so complex as to require a detailed explanation of its operations. 



\section{APPENDIX 2.A LISTING OF FORTRAN CODE ALPHA_MDA.FOR, FILE VERSION AS OF MARCH 12, 1996}

File is located on system $B$ disk at dubo: [000000.nd_root.ndamscust.for]

Tue Mar 12 09:04:38 EST 1996

\$ cat $-\mathrm{n}$ alfmdat 4 . for

$\mathrm{C}$

$\star * \star * *$ AIPHA MDA PROGRAM

C

ALPHA SPECTROMETRY SOFTWARE VERSION $1.023 \pi U 191$

C

PROGRAM [ALPHA.FOR] ALPHA_MDA.FOR

C

This program corrects activities by the amount the tracer is

off

It gets the isotope type from 'sample type'

and then decides which isotope is the tracer

It calculates the correction factor from this

and corrects the results accordingly

It also corrects the exrors

FIX BUG 12-MAR-1992, R.I.FURGUSON (MARTIN MARRIETTA - X10)

rsh 5/93 changes for LLNL and RJS

average eff digit was missing

yield digit was missing

adding lambda value to header

jxk 12/19/94-3/??/95 additions to calculate more modern MDA

and $\mathrm{LC}$ including information from background and blank history

c and LC

$27 \mathrm{C}$

jxk 12/19/94-3/??/95

28

29

\begin{tabular}{|c|c|c|}
\hline 30 & C & cld file now used in nd ams com: \\
\hline 31 & C & $\mathrm{p} 1=$ config, $/ \mathrm{b} \overline{\mathrm{kg}}=\mathrm{areas} /[\mathrm{no}$ trailer/header=reports.har \\
\hline 32 & $\mathbf{C}$ & / [no] tracer $[=x-n n n]$ \\
\hline 33 & c & \\
\hline 34 & c & define verb alpha_mda \\
\hline 35 & c & image nd_ams_com:ālpha_mda.exe \\
\hline 36 & c & parameter pl, value (required) \\
\hline 37 & c & qualifier header, value (required), nonnegatable \\
\hline 38 & $\mathbf{c}$ & qualifier trailer, negatable \\
\hline 39 & c & qualifier bkg, value (required), nonnegatable \\
\hline 40 & c & qualifier tracer, value (optional), negatable \\
\hline 41 & & \\
\hline
\end{tabular}


42

43

44

45

46

47

48

49

50

51

52

53

54

55

56

57

58

59

60

61

62

63

64

65

66

67

68

69

70

71

72

73

74

75

76

77

78

79

80

81

82

83

84

85

86

87

88

89

90

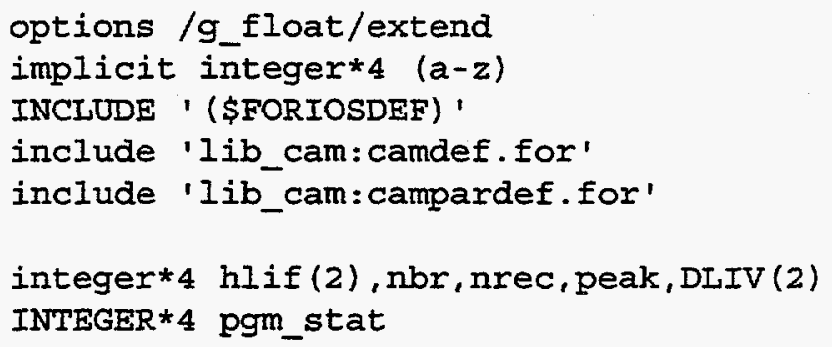


Appendix 2.A Listing of FORTRAN code ALPHA MDA.FOR, file version as of January 10, 1996

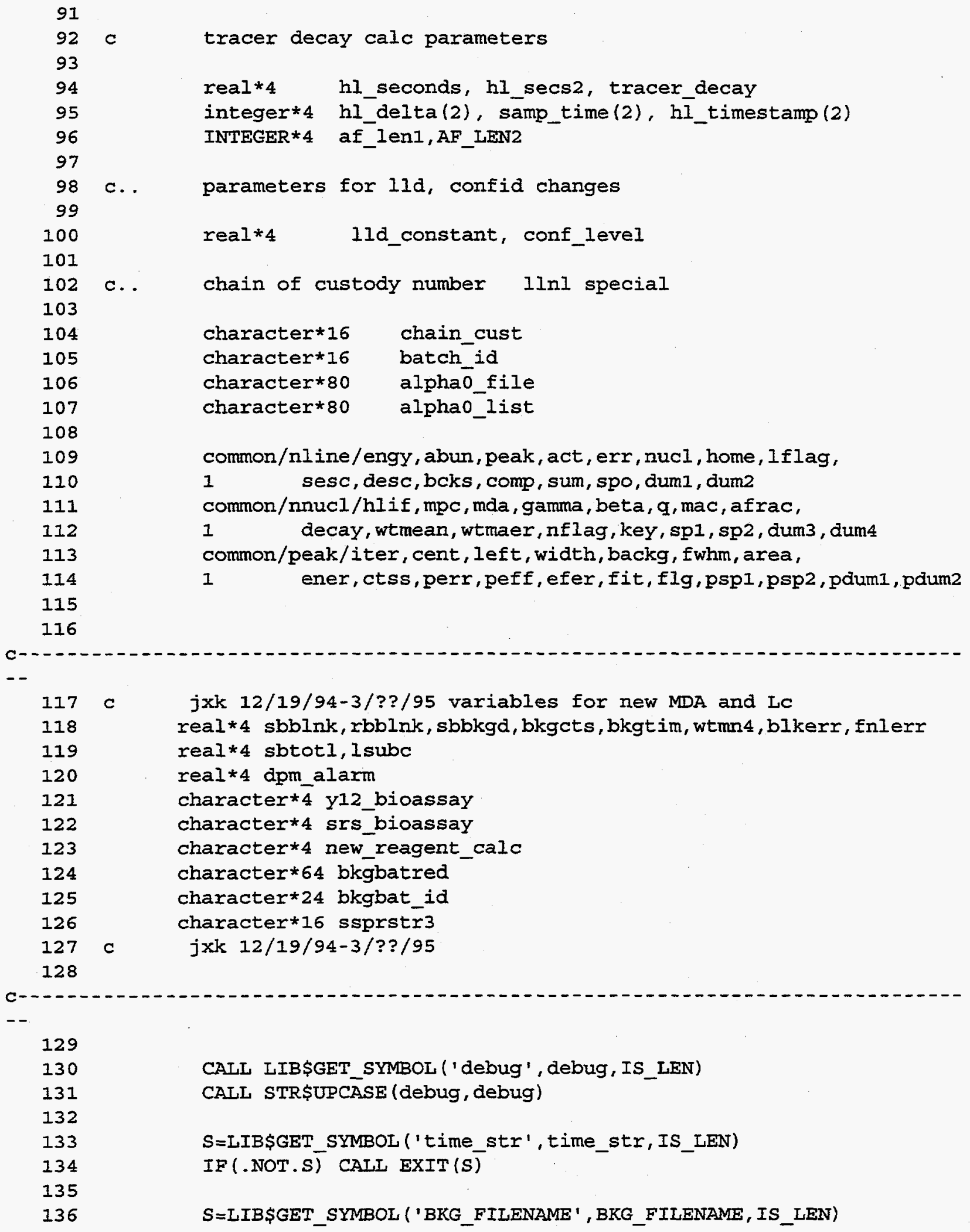

CALI LIB\$GET_SYMBOL ('debug', debug, IS_LEN)

CALL STRSUPCASE (debug, debug)

$S=$ IIB $\$ G E T$ SYMBOL ('time_str', time_str, IS_LEN)

IF(.NOT.S) CAII EXIT(S)

$S=L I B \$ G E T$ SYMBOL ('BKG_FILENAME' , BKG_FILENAME, IS_LEN) 
Appendix 2.A Listing of FORTRAN code ALPHA MDA.FOR, file version as of January 10, 1996

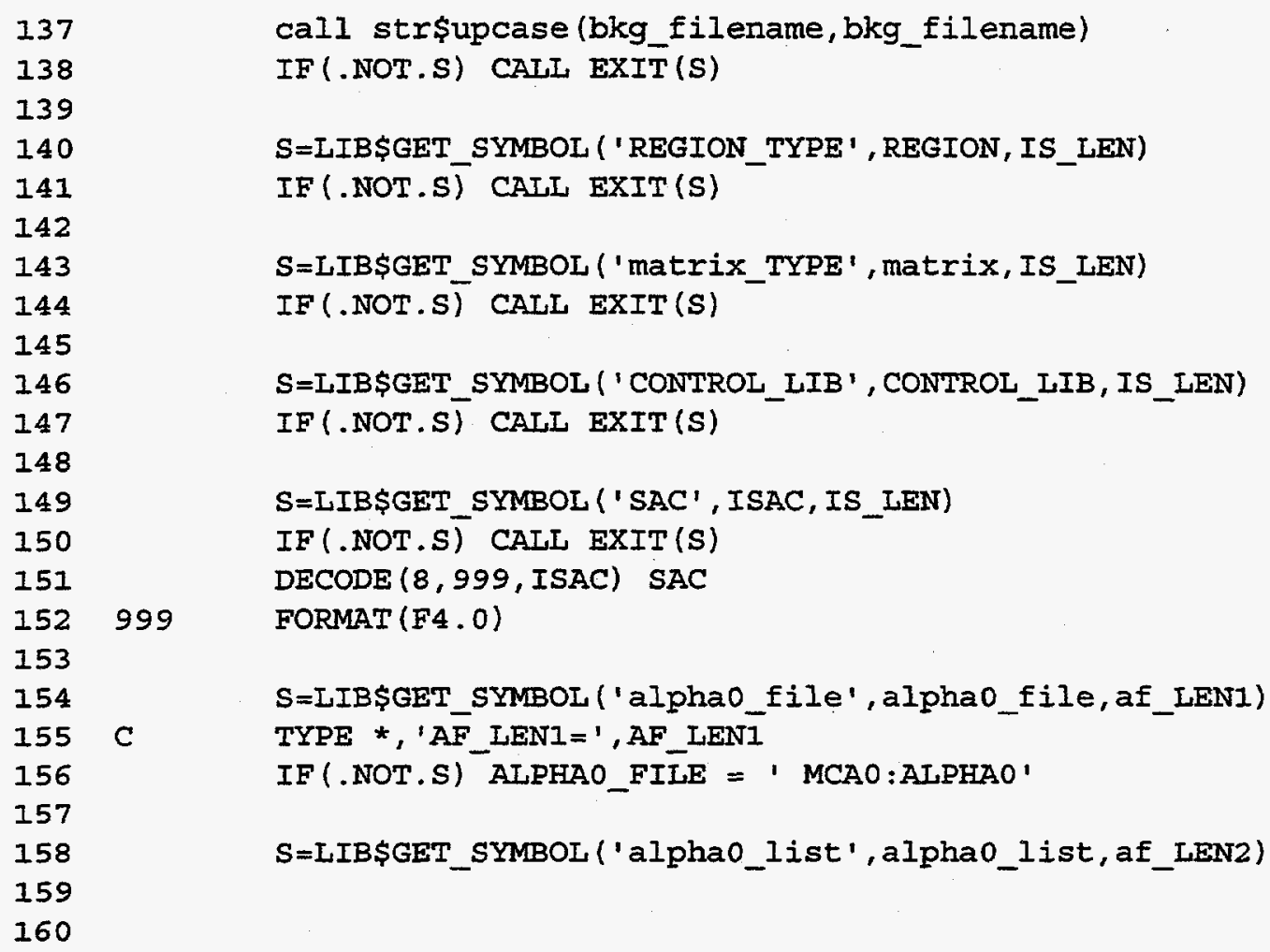




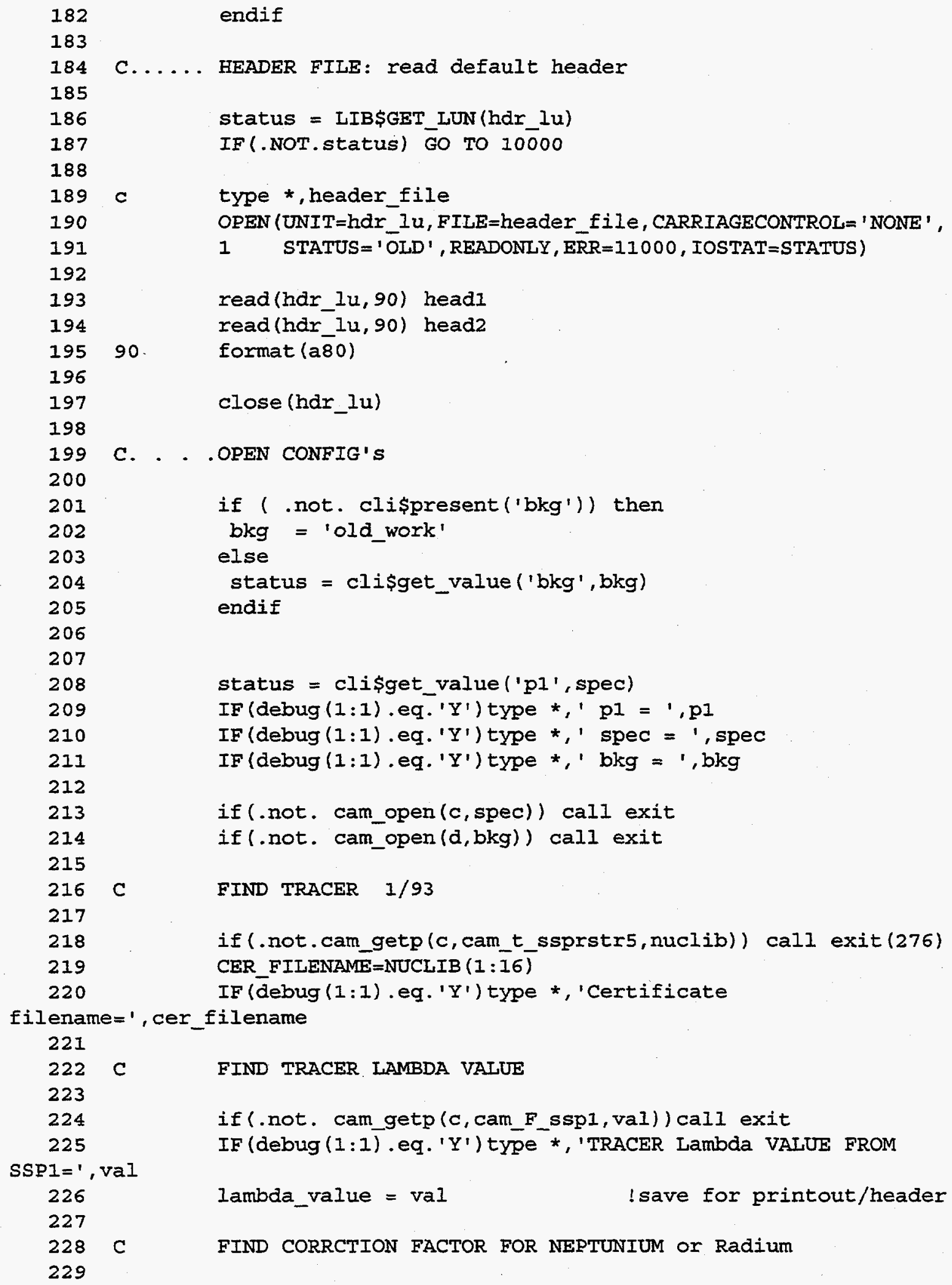


Appendix 2.A Listing of FORTRAN code ALPHA MDA.FOR, file version as of January 10, 1996

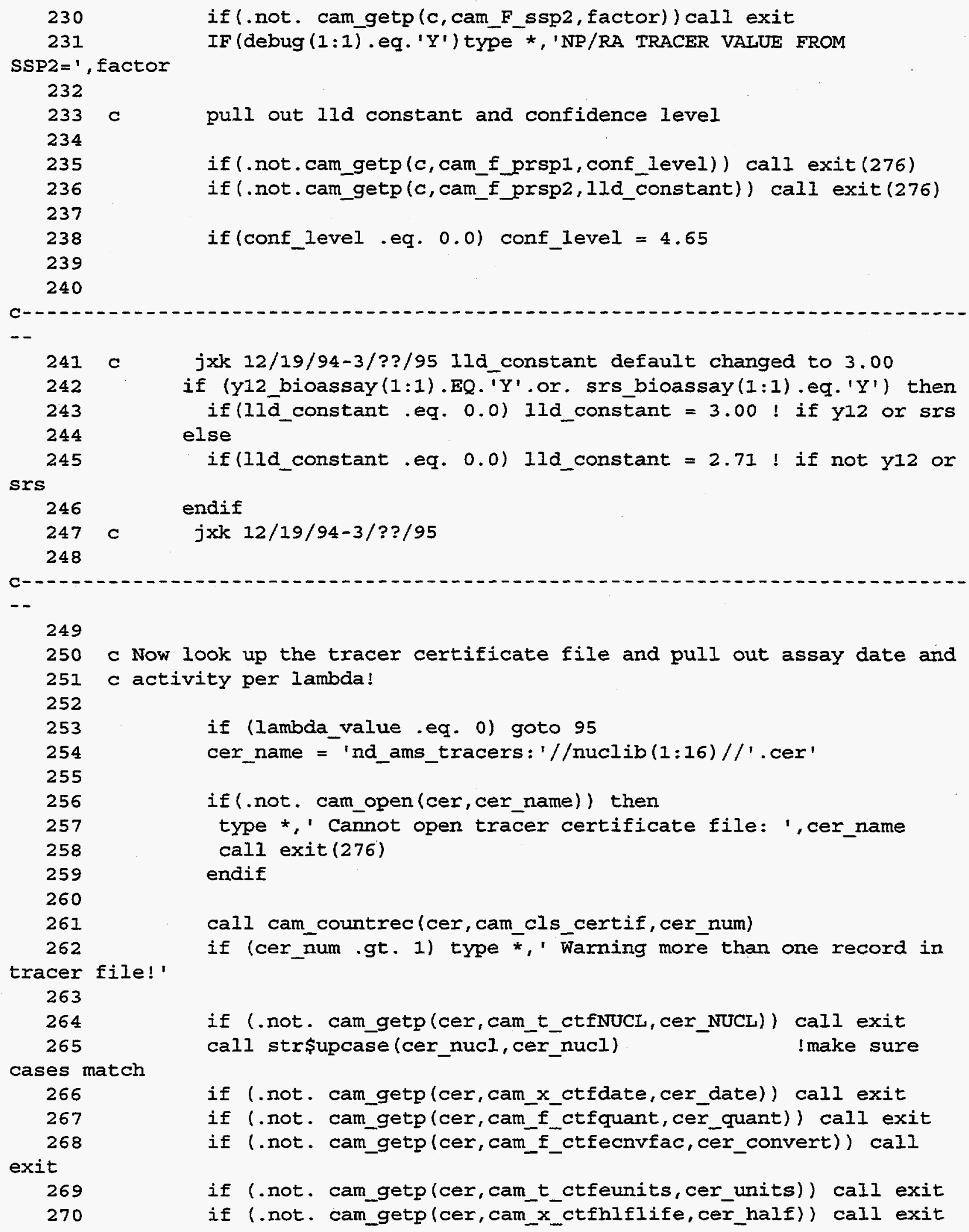


Appendix 2.A Listing of FORTRAN code ALPHA MDA.FOR, file version as of January 10.1996

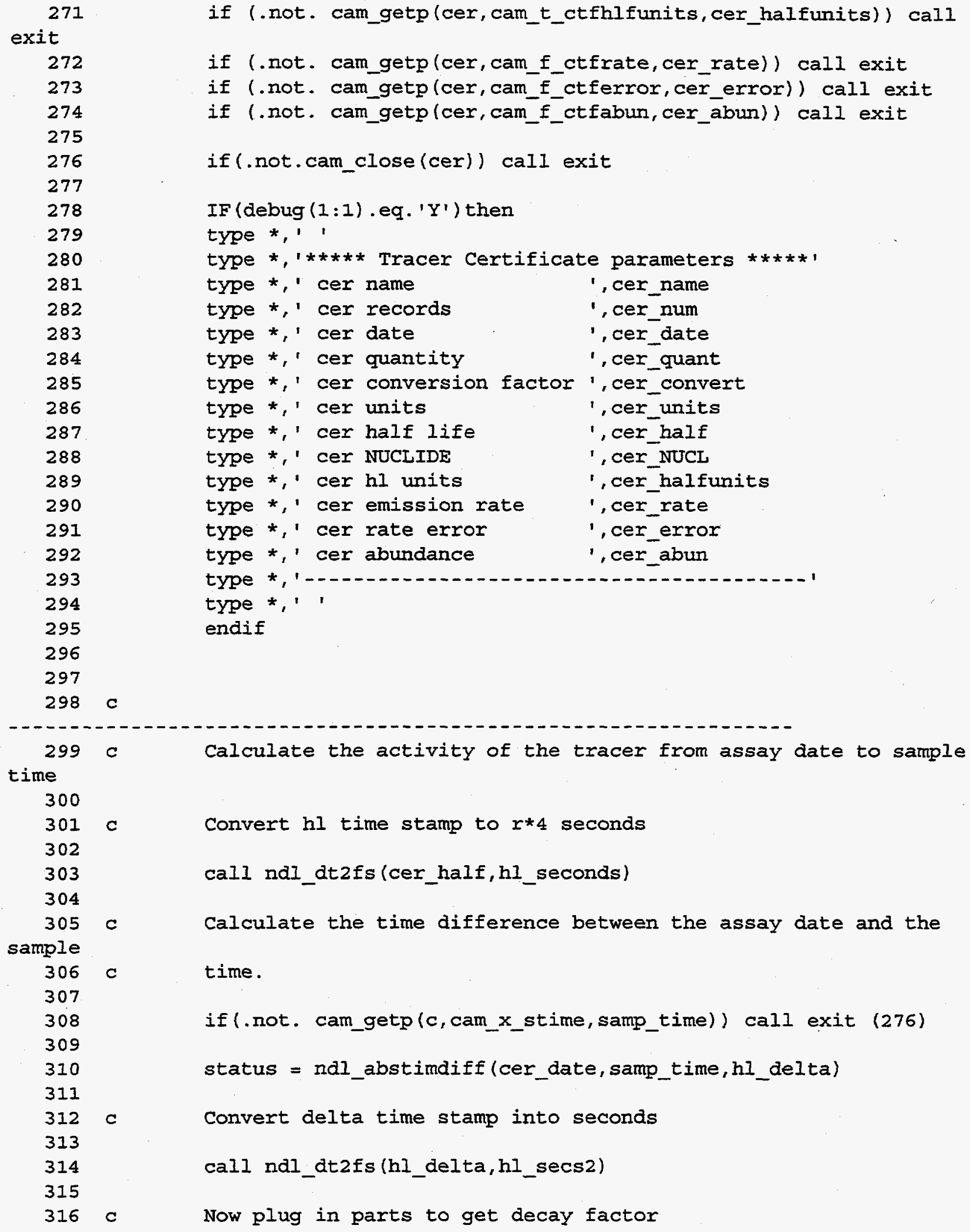


Appendix 2.A Listing of FORTRAN code ALPHA MDA.FOR, file version as of January 10, 1996

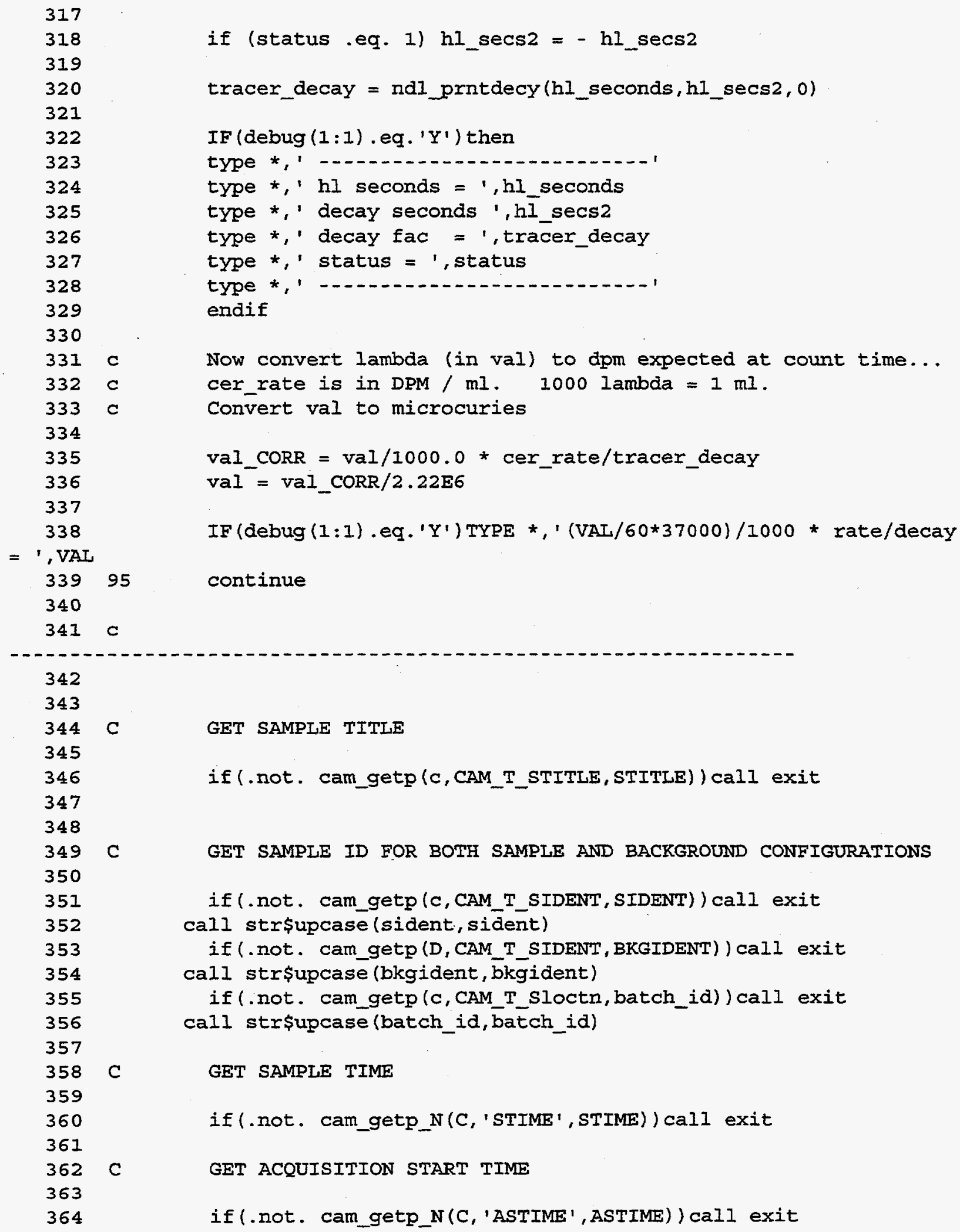


Appendix 2.A Listing of FORTRAN code ALPHA _MDA.FOR, file version as of January 10, 1996

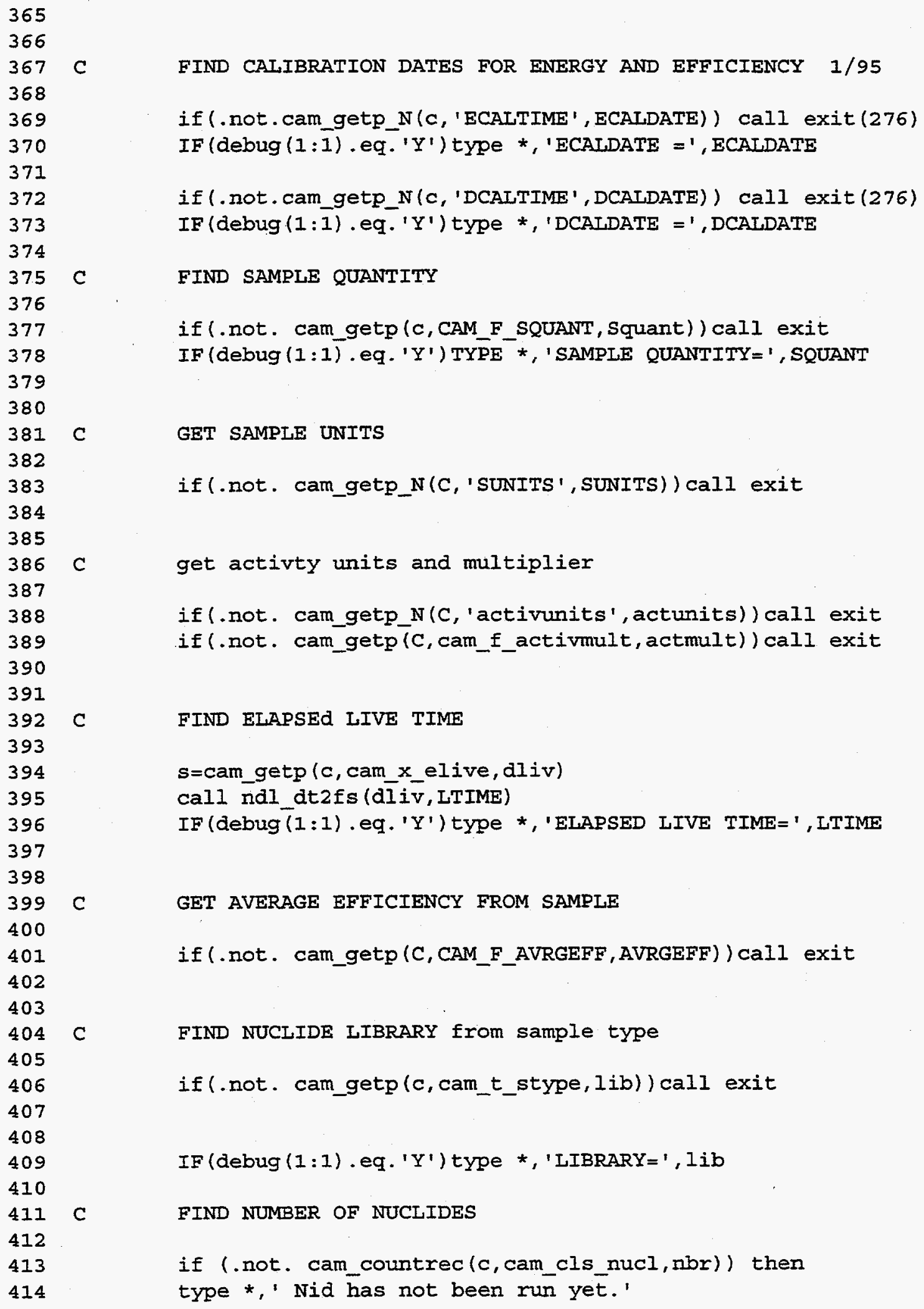


Appendix 2.A Listing of FORTRAN code ALPHA MDA.FOR, file version as of January 10.1996

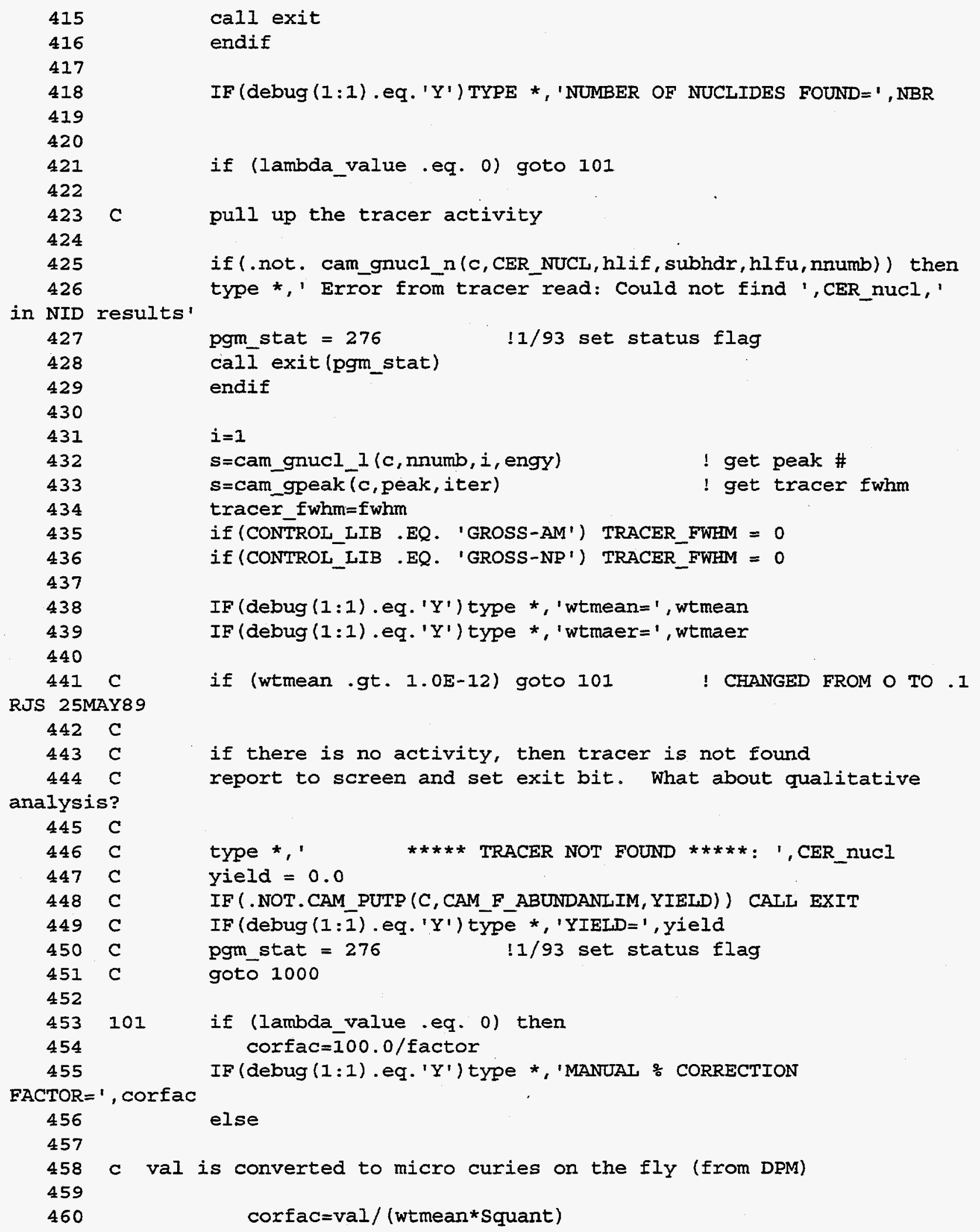


Appendix 2.A Listing of FORTRAN code ALPHA MDA.FOR, file version as of January 10, 1996

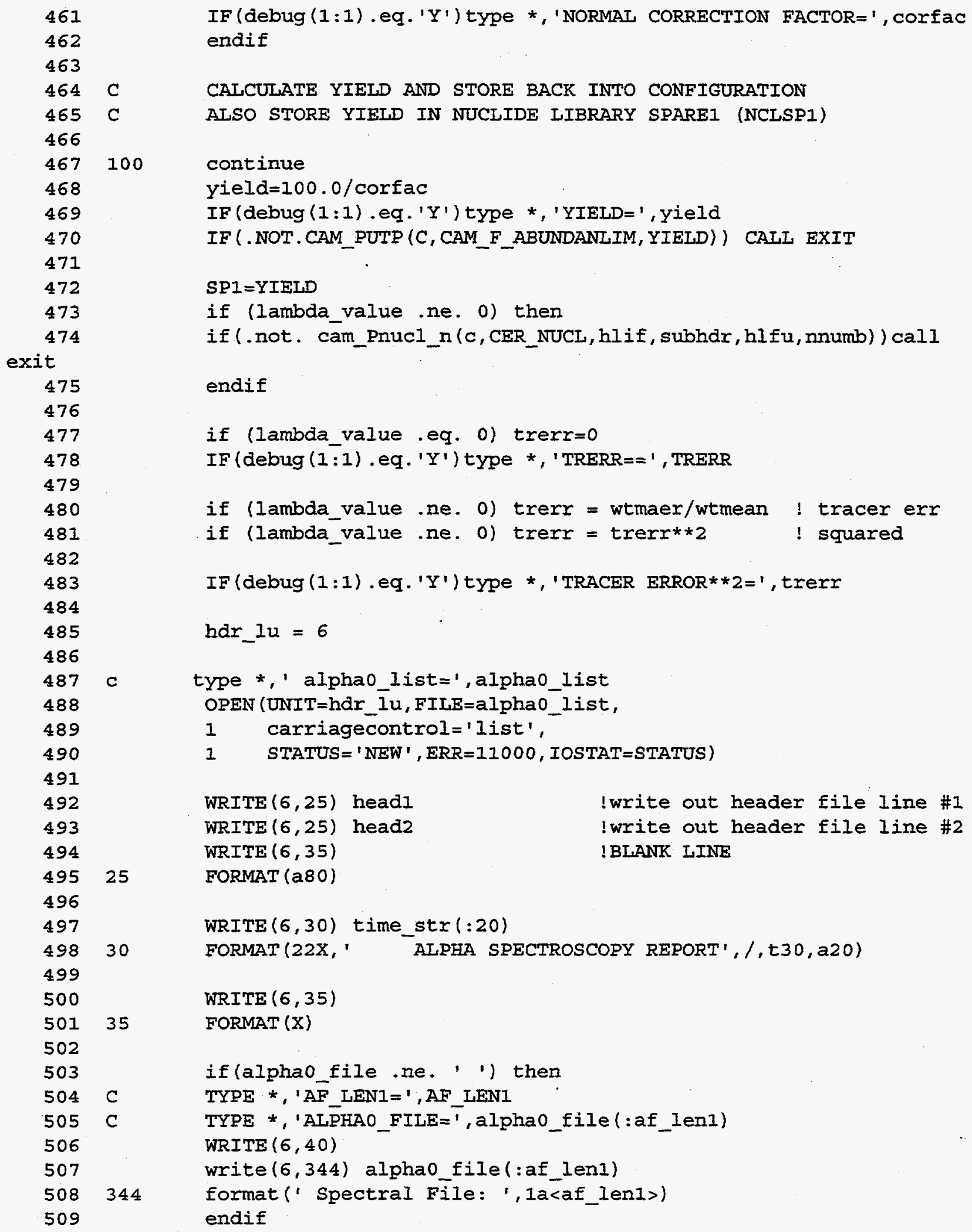

461

462

463

$464 \mathrm{C}$

$465 \mathrm{C}$

466

467

468

469

470

471

472

473

474

exit

475

476

477

478

479

480

481

482

483

484

485

486

487

488

489

490

491

492

493

494

495

496

497

498

499

500

501

502

503

504

505

506

507

$508 \quad 344$

509

IF (debug $(1: 1$ ) .eq. ' $\mathrm{Y}$ ') type *, 'NORMAI CORRECTION FACTOR=', corfac endif

CALCULATE YIELD AND STORE BACK INTO CONFIGURATION

C ALSO STORE YIELD IN NUCLIDE LIBRARY SPAREI (NCLSPI)

100 continue

yield $=100.0 /$ corfac

IF (debug ( $1: 1$ ) .eq. 'Y') type *, 'YIELD=' , yield

IF (.NOT.CAM_PUTP (C, CAM_F_ABUNDANLIM, YIELD)) CALL EXIT

$S P I=Y I E L D$

if (lambda value ne. 0) then

if (. not. cam_pnucl_n(c, CER_NUCL, hlif, subhdr, hlfu, nnumb)) call

endif

if (lambda_value .eq. 0) trerr $=0$

IF (debug $(1: 1)$.eq. ' $Y$ ') type *, 'TRERR== ' , TRERR

if (lambda_value ne. 0) trerr = wtmaer/wtmean ! tracer err

if (lambda_value ne. 0) trerr $=$ trerr**2 ! squared

IF (debug (1:1) .eq. ' $Y$ ') type *, 'TRACER ERROR**2=', trerr

har_lu $=6$

c type *,' alphao_list=', alphao_list

OPEN (UNIT $=$ hdr_ $\bar{l} u$, FILE=alphao_list,

1 carriagecontrol='list',

1 STATUS = 'NEW' , ERR=11000, IOSTAT=STATUS )

WRITE $(6,25)$ headl

WRITE $(6,25)$ head2

WRITE $(6,35)$

!write out header file line \#I

FORMAT (a80)

!write out header file line \#2

!BLANK IINE

25

WRITE $(6,30)$ time_str $(: 20)$

30

FORMAT (22X, '

AIPHA SPECTROSCOPY REPORT', (,t30, a20)

WRITE $(6,35)$

FORMAT (X)

if (alphaofile .ne. ' ') then

C TYPE *, 'AF_LEN1=',AF_LEN1
C TYPE *, 'ALPHAO_FILE $=$ ', alphao_file (:af_lenl)

$\operatorname{WRITE}(6,40)$

write (6,344) alphao_file(:af_lenI)

344 format (' Spectral File: ', 1a<af_lenI>)

endif 


\section{2-34}

Appendix 2.A Listing of FORTRAN code ALPHA MDA.FOR, file version as of January 10, 1996

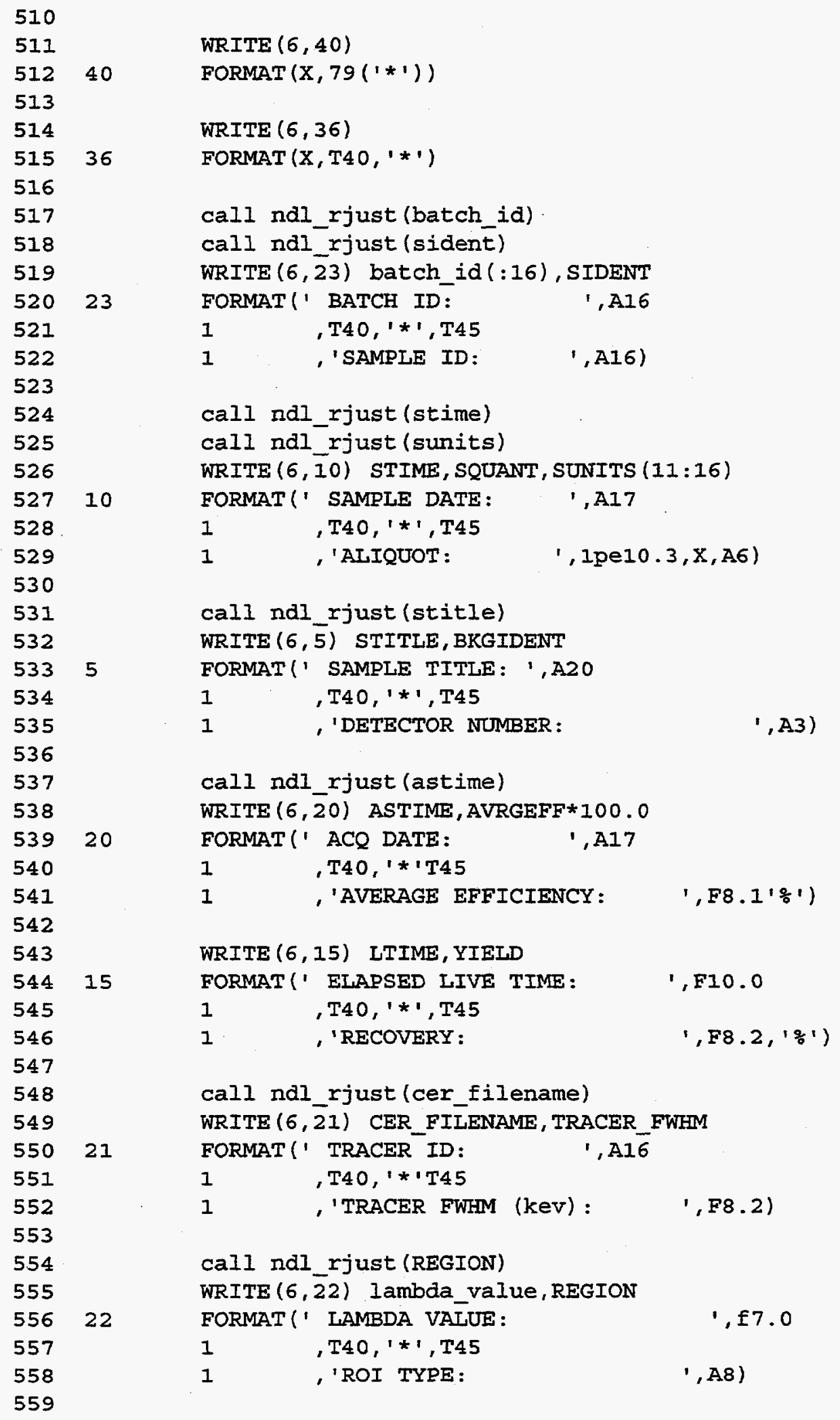




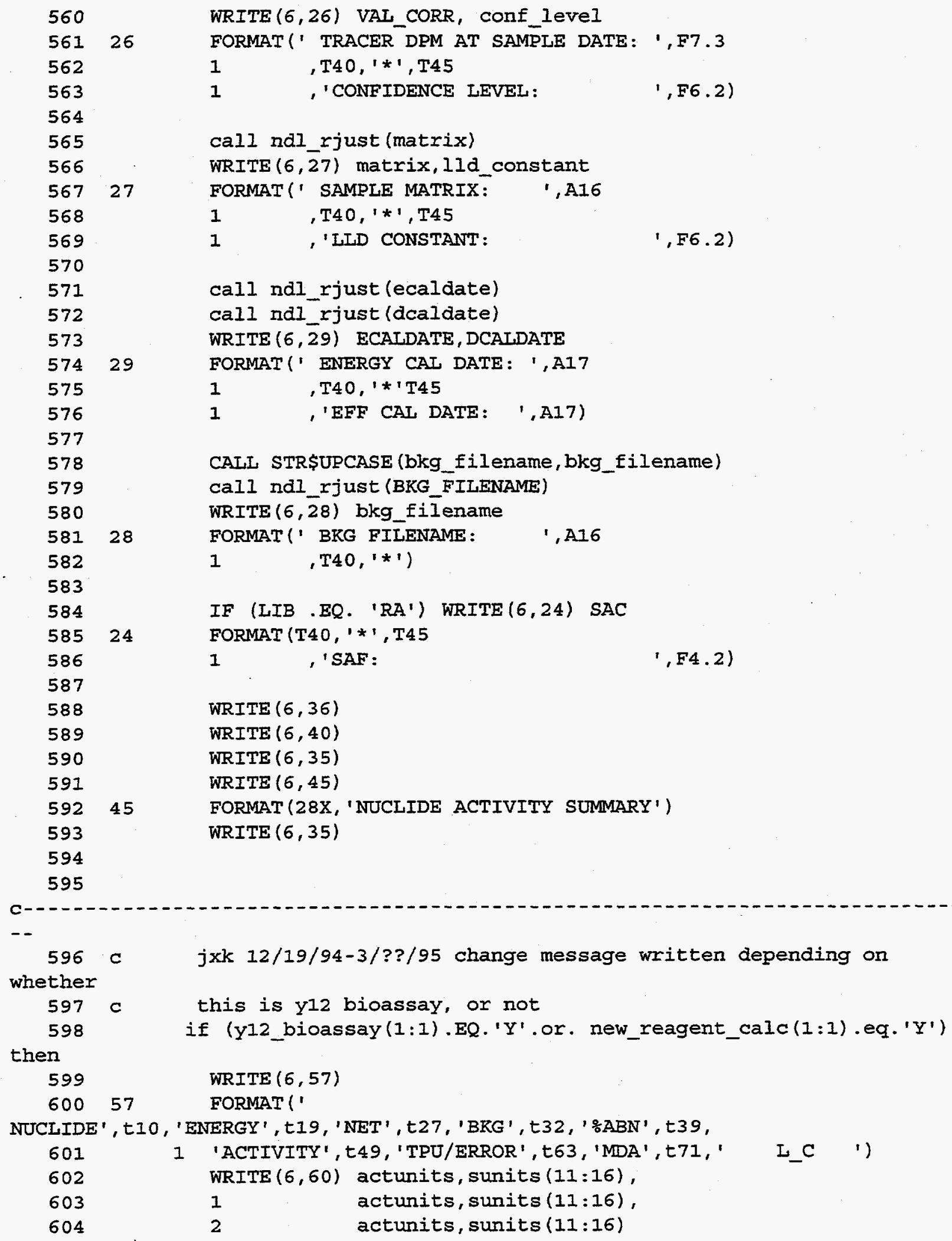


Appendix 2.A Listing of FORTRAN code ALPHA MDA.FOR, file version as of January 10, 1996

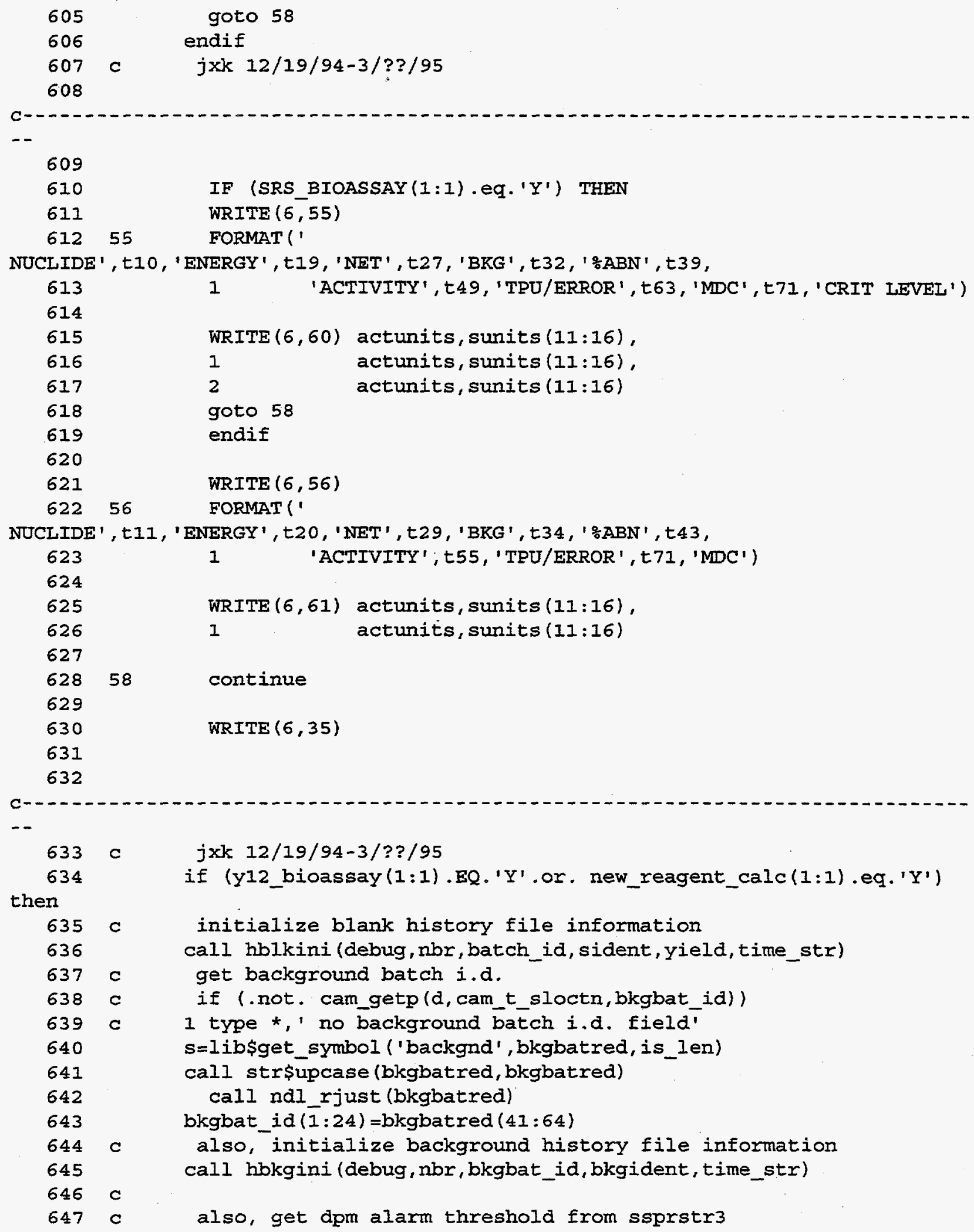


Appendix 2.A Listing of FORTRAN code ALPHA MDA.FOR, file version as of January 10. 1996

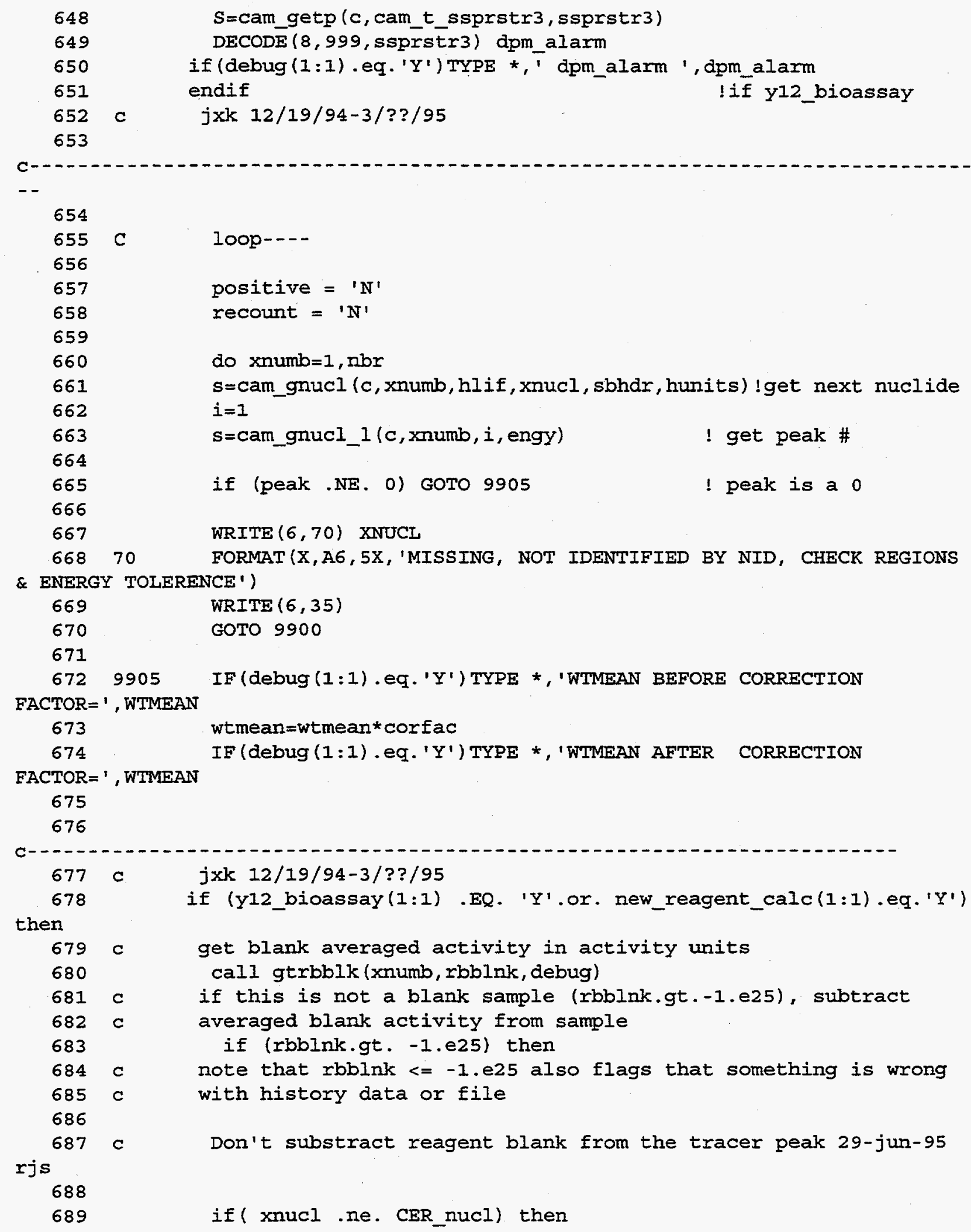




\section{2-38}

Appendix 2.A Listing of FORTRAN code ALPHA MDA.FOR, file version as of January 10, 1996

690

691

692

693

694

695

696

697

$698 \mathrm{C}$

$699 \mathrm{C}$

$700 \mathrm{C}$

701

702

', dum3

703

704

705

706 wtmean=wtmean $-r b b l n k$

endif

IF (debug ( $I: 1$ ) .eq. ' $Y$ ') then

TYPE *, 'WTMEAN AFTER CORRECTION for blank',

1 ' activity $=1$, WTMEAN

type *,' avg blank activity ', rbblnk endif ! debug

blank activity needs to be stored in some spare string.

convert to a coded integer and store in memory location for spare string dum3

call reinpak (rbblnk, dum3)

if(debug(1:1) .eq. ' $Y$ ') type *,' coded integer for blank activity

$$
\text { endif }
$$

endif

c jxk 12/19/94-3/??/95

!if rbblnk.gt. $-1 . e 25$

!if y12_bioassay

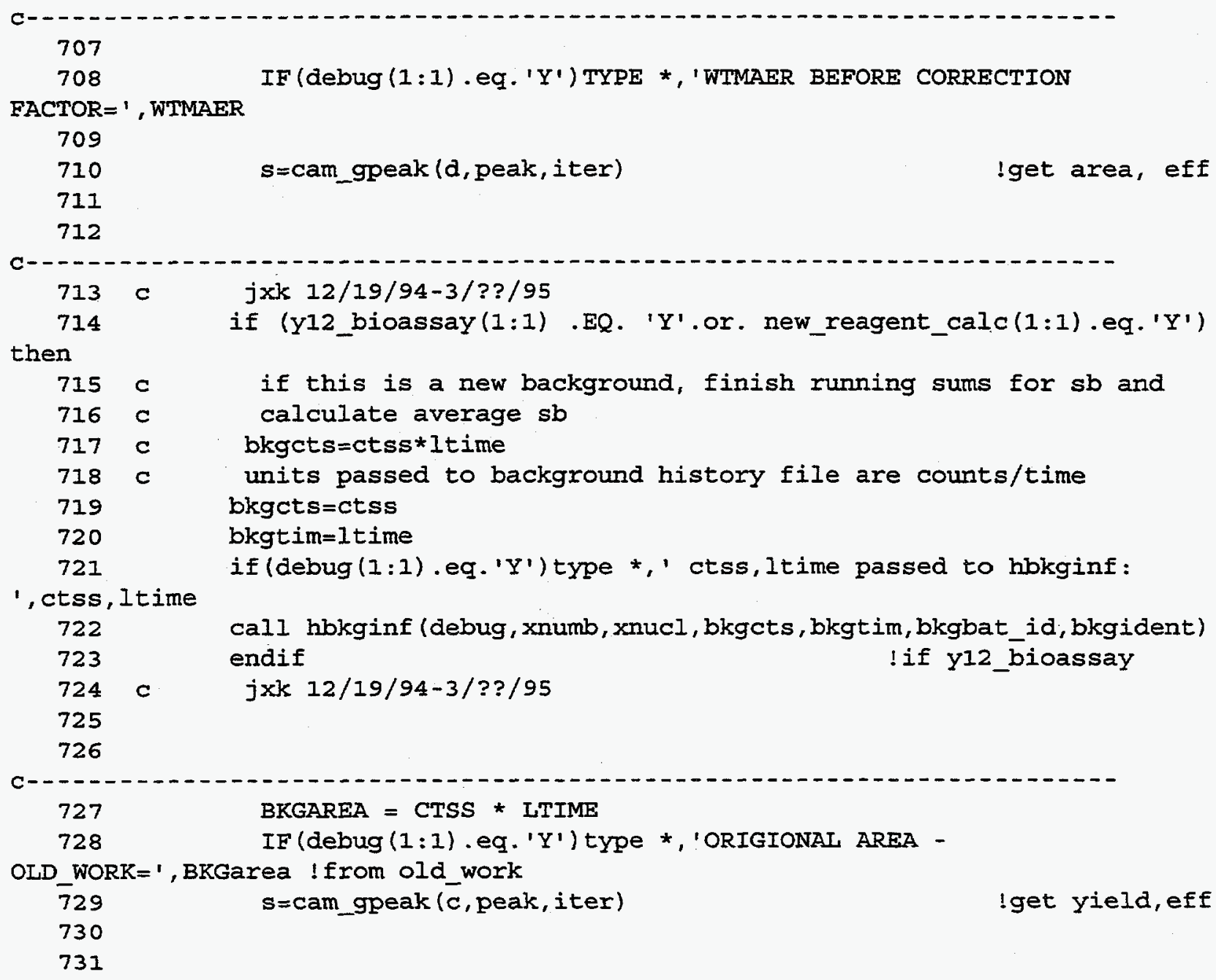


Appendix 2.A Listing of FORTRAN code ALPHA MDA.FOR, file version as of January 10, 1996

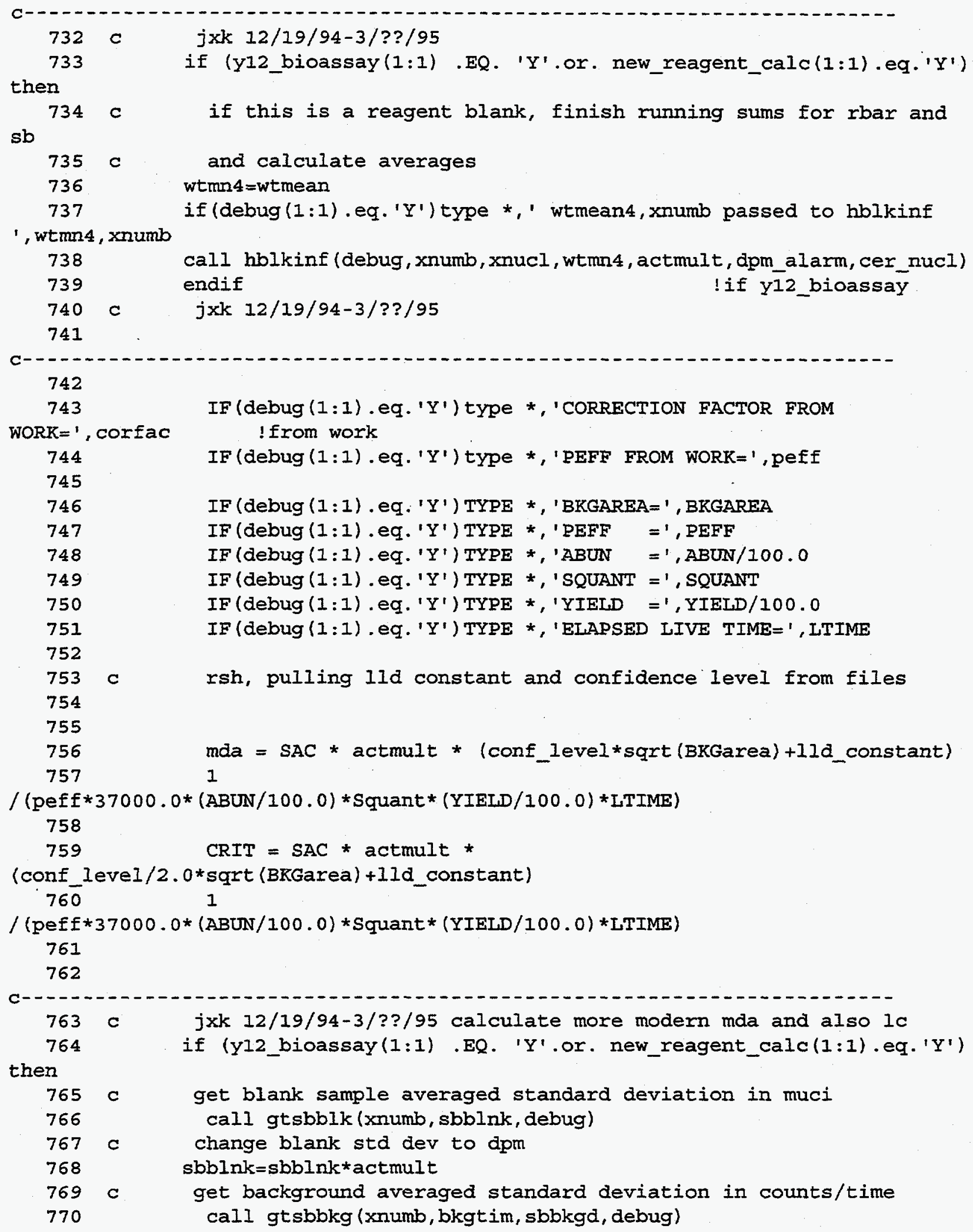


Appendix 2.A Listing of FORTRAN code ALPHA MDA.FOR, file version as of January 10, 1996

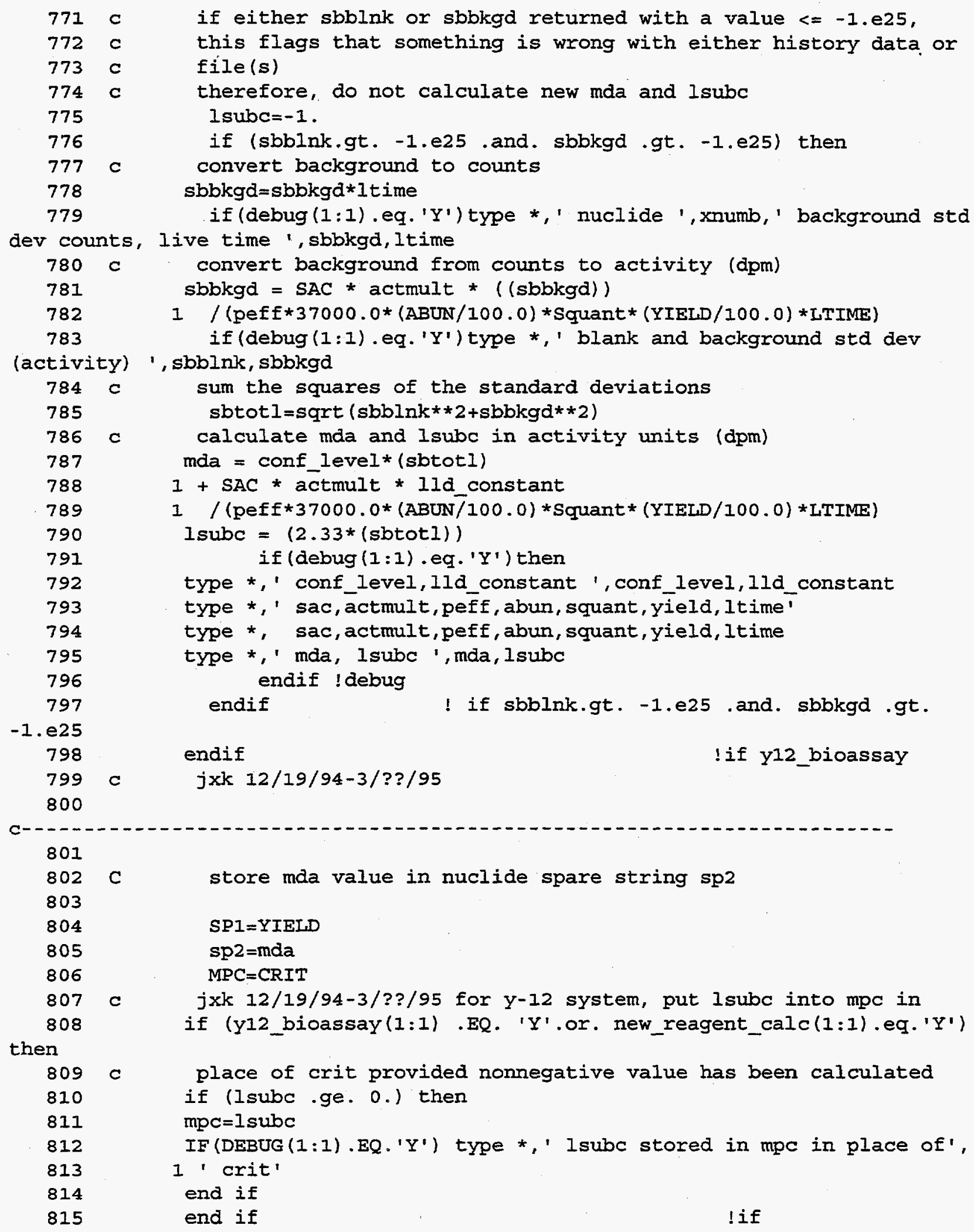




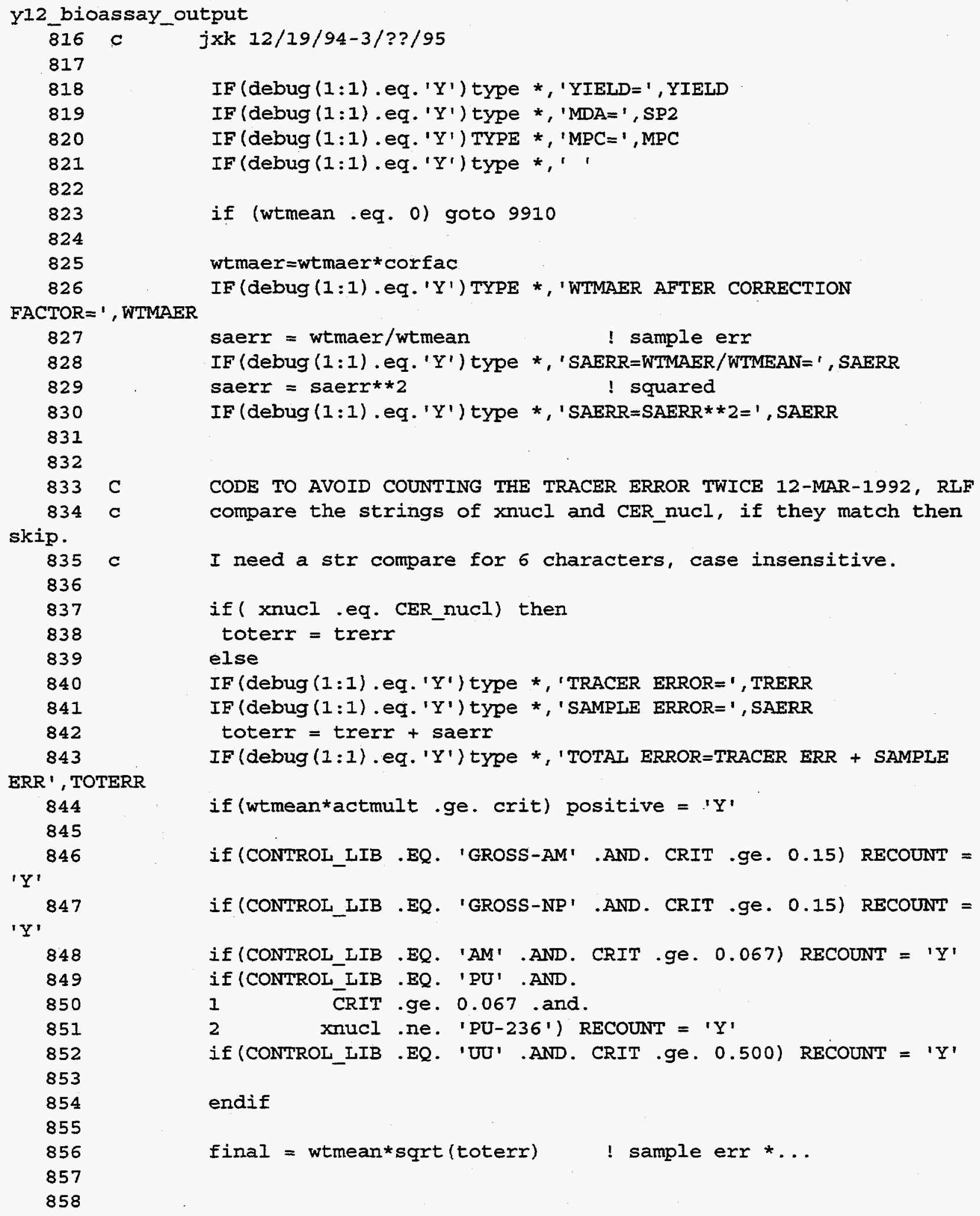


Appendix 2.A Listing of FORTRAN code ALPHA MDA.FOR, file version as of January 10, 1996

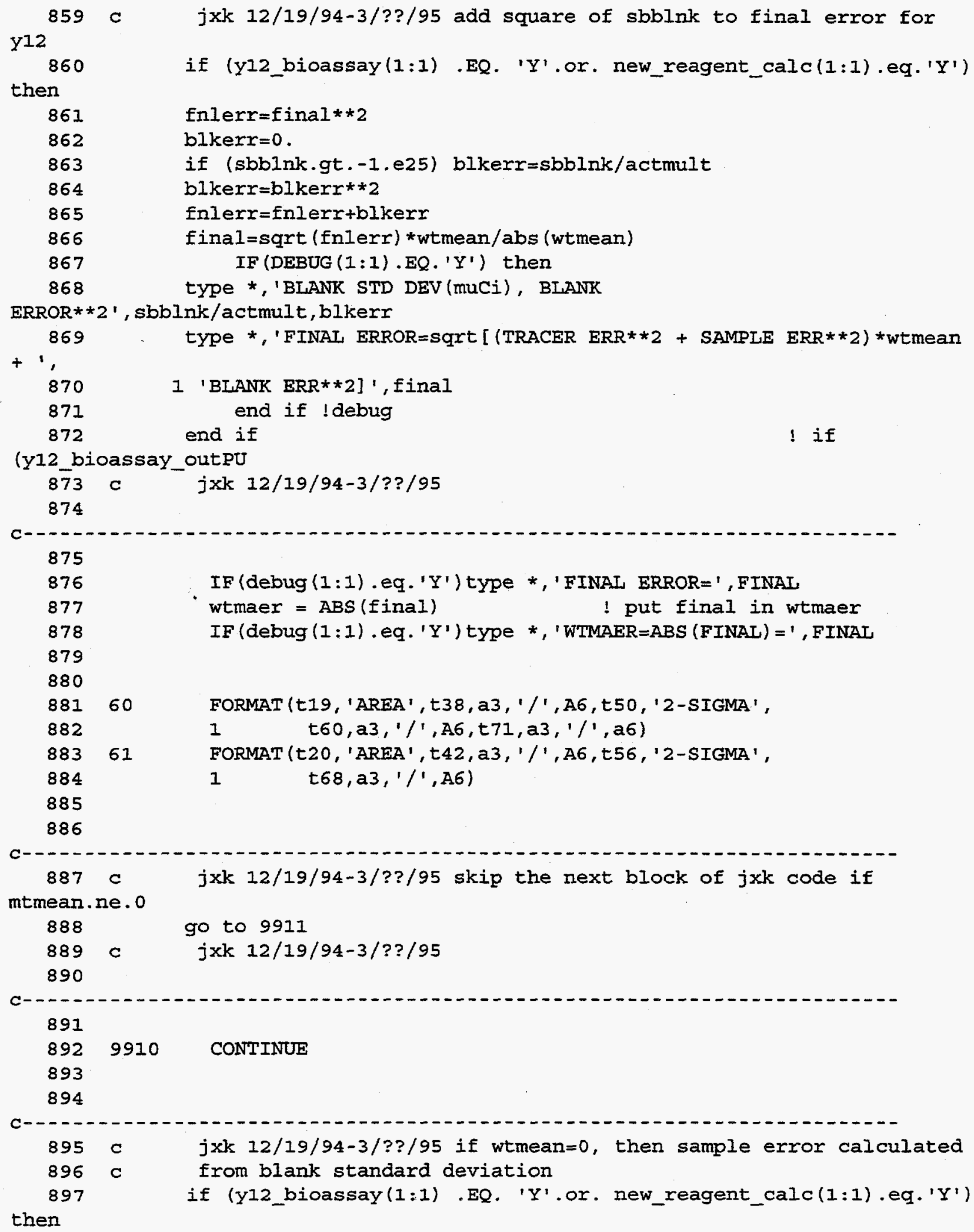




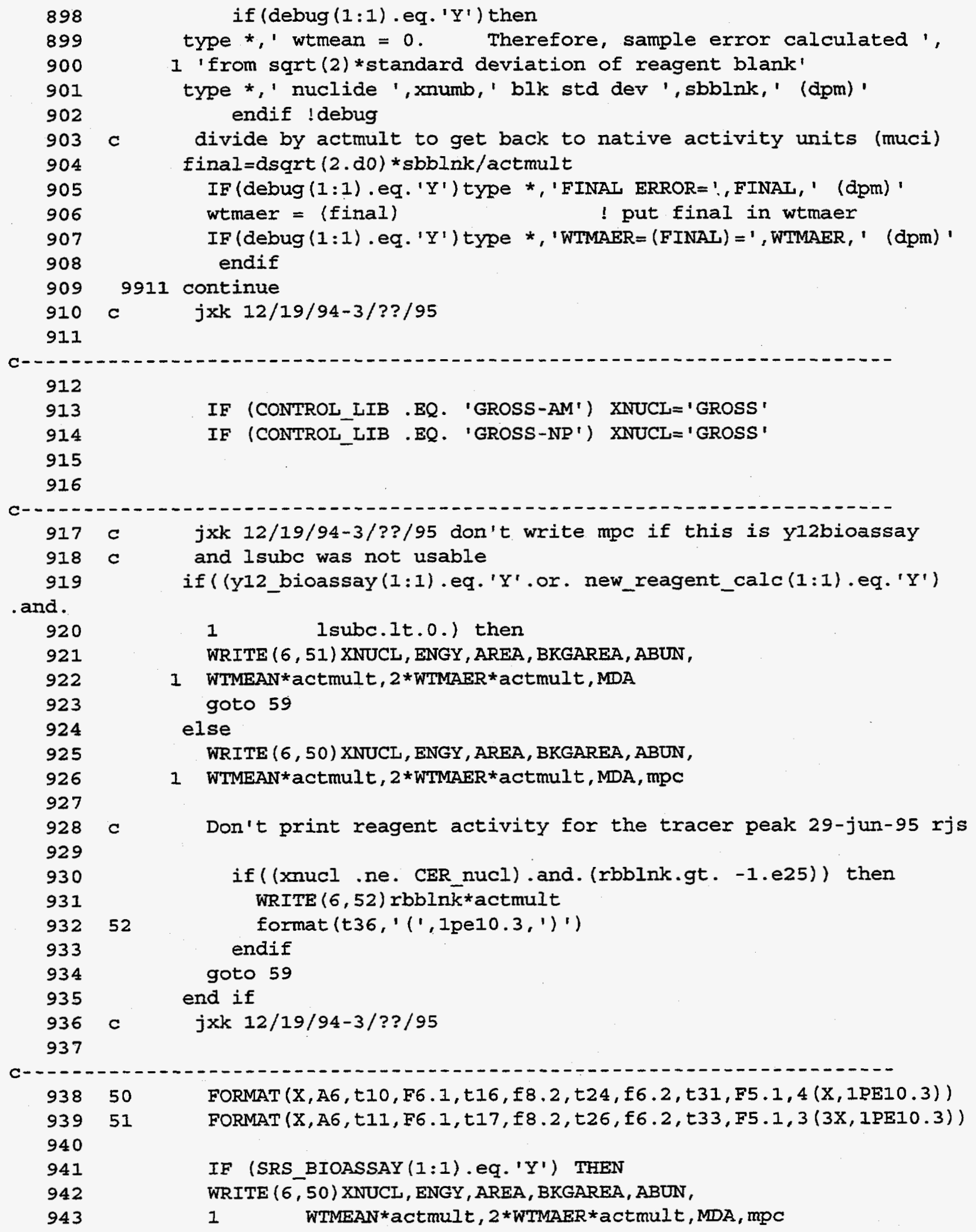

9911 continue

c jxk 12/19/94-3/??/95

final =dsqrt (2.do)*sbblnk/actmult IF (debug $(1: 1)$. eq. ' $Y$ ') type *, 'FINAI ERROR=', FINAI, ' (apm)' wtmaer = (final) $\quad$ put final in wtmaer IF (debug $(1: 1)$. eq. ' $Y$ ') type *, 'WTMAER $=($ FINAL) $=$ ', WTMAER, ' (dpm) ' endif

IF (CONTROL_LIB .EQ. 'GROSS-NP') XNUCL=' GROSS'

jxk 12/19/94-3/??/95 don't write mpc if this is yl2bioassay

C and lsubc was not usable if ( (Y12_bioassay $(1: 1)$.eq. ' $Y$ ' or. new_reagent_calc $(1: 1)$.eq. 'Y') 
Appendix 2.A Listing of FORTRAN code ALPHA_MDA.FOR, file version as of January 10, 1996

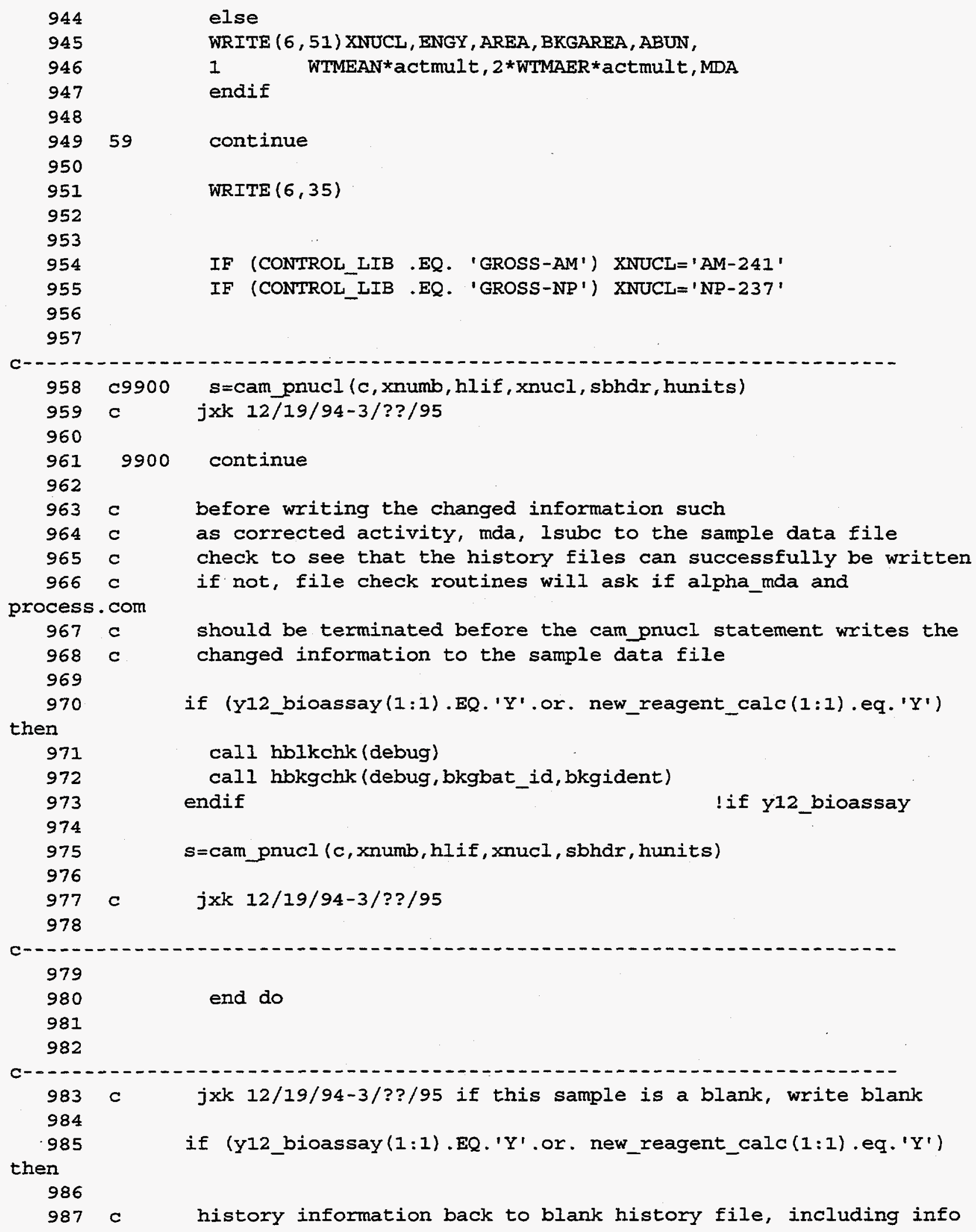


Appendix 2.A Listing of FORTRAN code ALPHA MDA.FOR, file version as of January 10. 1996

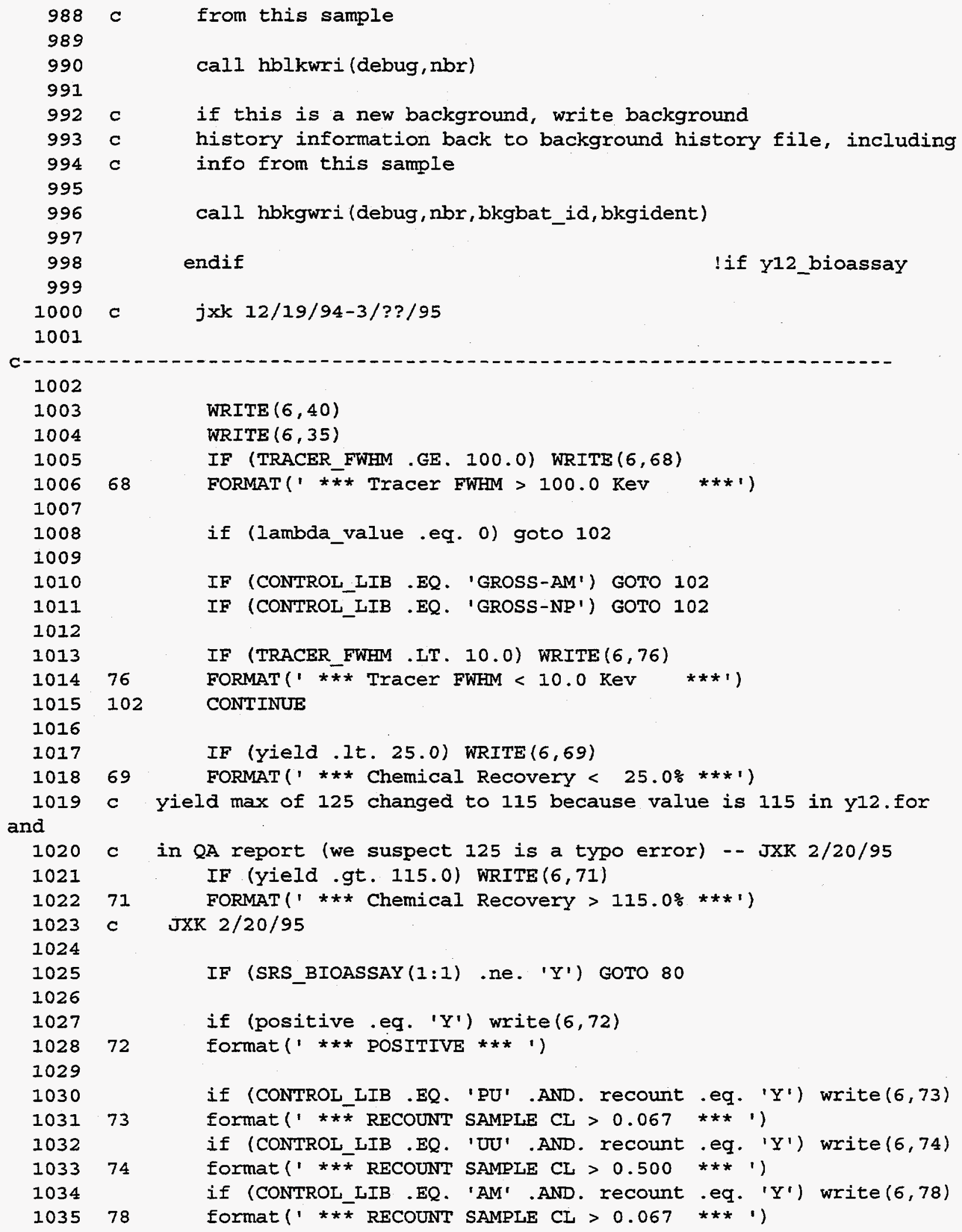


Appendix 2.A Listing of FORTRAN code ALPHA MDA.FOR, file version as of January 10, 1996

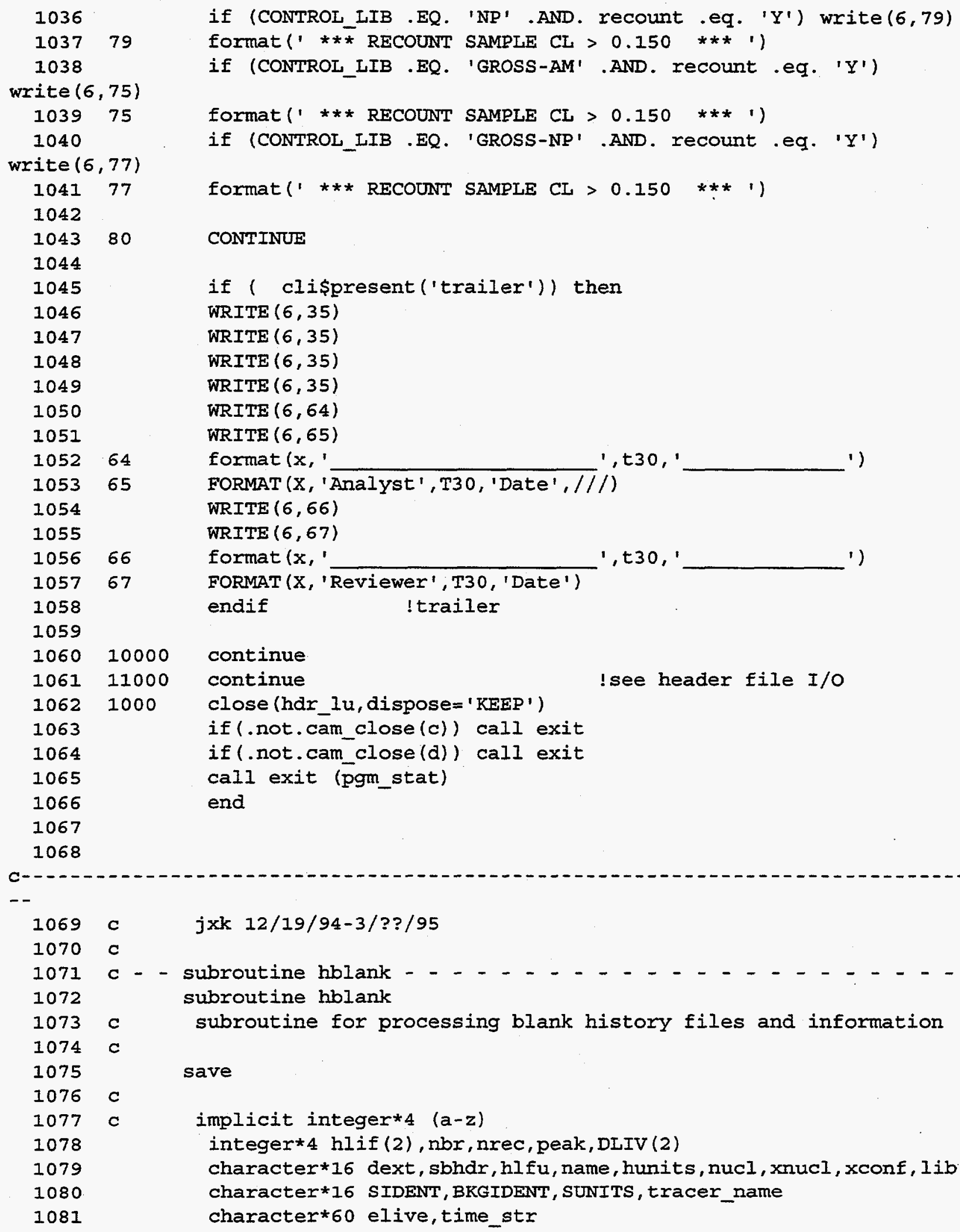


Appendix 2.A Listing of FORTRAN code ALPHA MDA.FOR, file version as of January 10, 1996

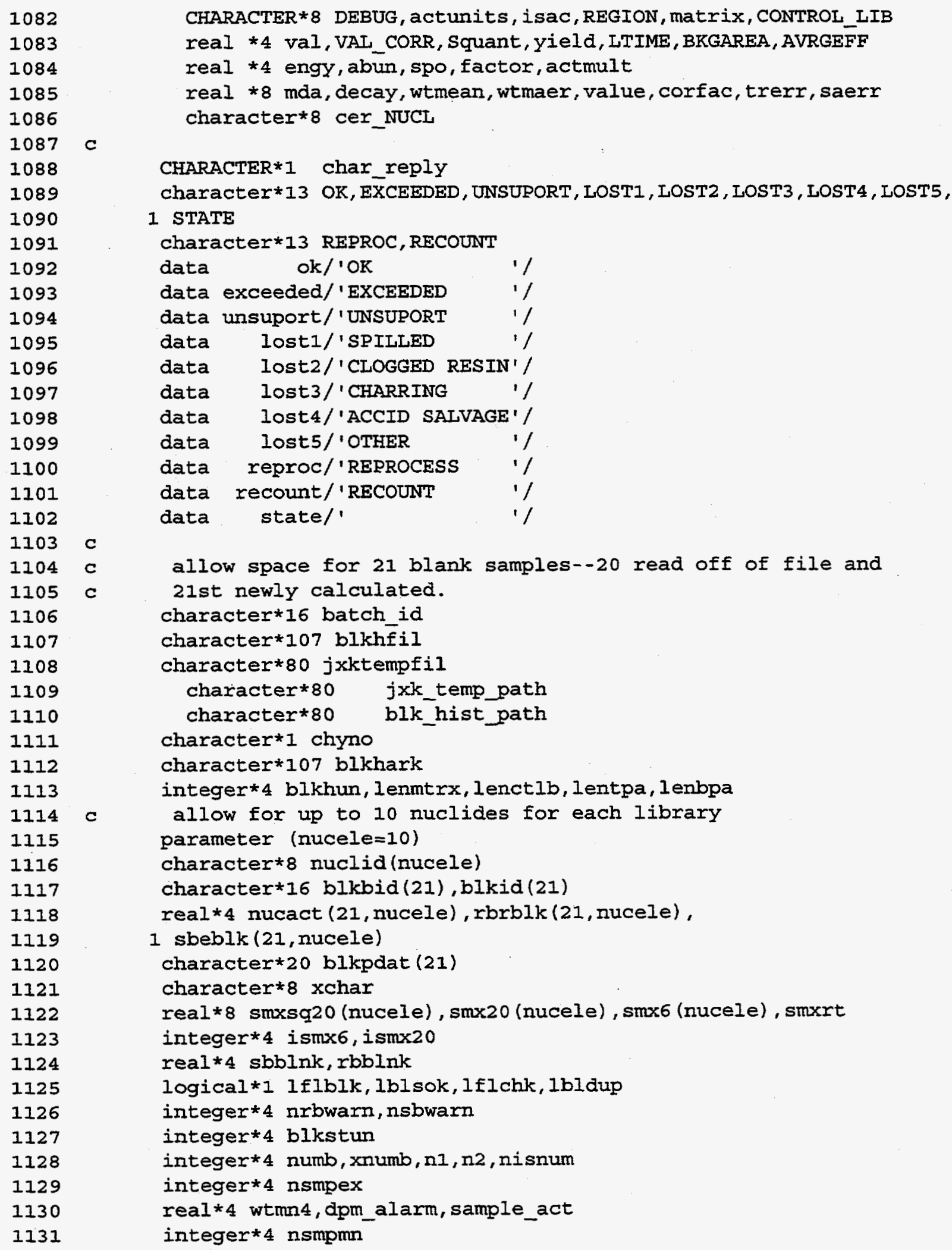

1082

1083

1084

1085

1086

1087

1088

1089

1090

1091

1092

1093

1094

1095

1096

1097

1098

1099

1100

1101

1102

1103

1104

1105

1106

1107

1108

1109

1110

1111

1112

1113

1114

1115

1116

1117

1118

1119

1120

1121

1122

1123

1124

1125

1126

1127

1128

1129

1130

1131

CHARACTER *8 DEBUG, actunits, isac, REGION, matrix, CONTROL LIB real *4 val, VAI_CORR, squant, yield, LTIME, BKGAREA, AVRGEFF real *4 engy, abun, spo, factor, actmult

real $\star 8 \mathrm{mda}$, decay, wtmean, wtmaer, value, corfac, trerr, saerr character*8 cer_NUCL

c

CHARACTER*I char_reply

character*13 OK, EXCEEDED, UNSUPORT, LOST1, LOST2, LOST3, LOST4, LOST5,

1 STATE

character*13 REPROC, RECOUNT

data ok/'OK '/

data exceeded/'EXCEEDED $1 /$

data unsuport/'UNSUPORT $1 /$

data losti/'SPIILED $1 /$

data lost2/'CLOGGED RESIN'/

data lost3/'CHARRING $\%$

data lost4/'ACCID SAIVAGE'/

data lost5/'OTHER '/

data reproc/'REPROCESS '/

data recount/'RECOUNT '/

data state/' ' '/

C

c allow space for 21 blank samples--20 read off of file and

c 21st newly calculated.

character*16 batch_id

character*107 blkhfil

character*80 jxktempfil

character*80 jxk_temp_path

character*80 blk_hist path

character*1 chyno

character*107 blkhark

integer* 4 blkhun, lenmtrx, lenctlb, lentpa, lenbpa

c allow for up to 10 nuclides for each library

parameter (nucele $=10$ )

character*8 nuclid(nucele)

character*16 blkbid(21), blkid(21)

real*4 nucact $(21$, nucele $), \operatorname{rbrblk}(21$, nucele $)$,

$1 \operatorname{sbeblk}(21$, nucele)

character*20 blkpdat (21)

character*8 xchar

real*8 smxsq20 (nucele), smx20 (nucele), smx6 (nucele), smxrt

integer*4 ismx6, ismx20

real 4 sbblnk, rbblnk

logical*I lflblk, Iblsok, Iflchk, Ibldup

integer*4 nrbwam, nsbwarn

integer*4 blkstun

integer*4 numb, xnumb, $n 1, n 2, n i s n u m$

integer* 4 nsmpex

real*4 wtmn4, dpm_alarm, sample_act

integer*4 nsmpmn 
Appendix 2.A Listing of FORTRAN code ALPHA MDA.FOR, file version as of January 10, 1996

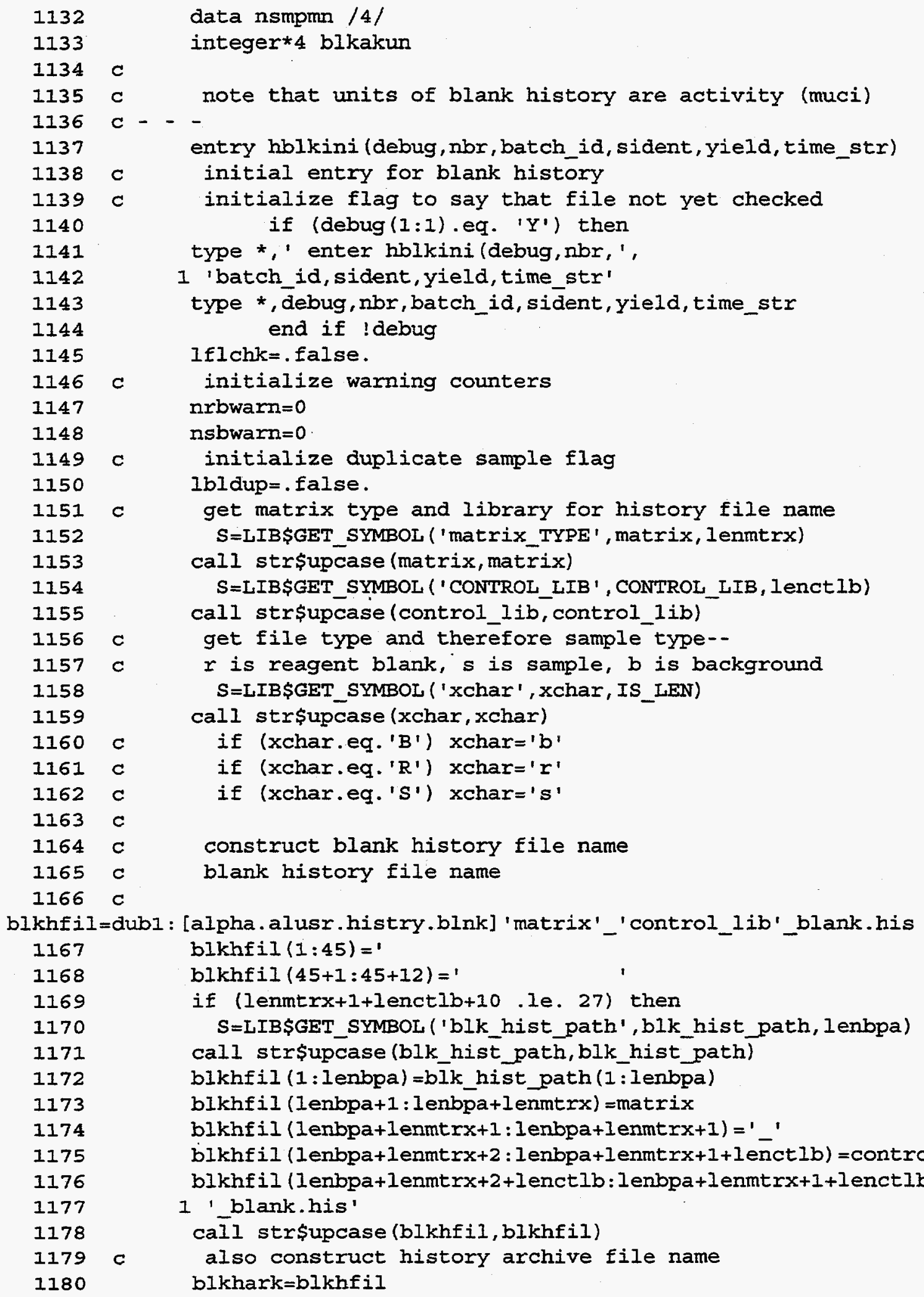


1181

1182

1183

1184

1185

1186

1187

1188

1189

1190

1191

1192

blkhark ( 1 enbpa + lenmt $r x+1+$ lenct $1 b+8: 1$ enbpa +1 enmt $r x+1+1$ enct $1 b+10)=$ 1 'ark'

call str\$upcase (blkhark, blkhark)

blkhun=99

blkakun $=96$

if (debug $(1: 1)$.eq. ' $Y$ ') then

type *,' blank history file to open'

type *,blkhfil

type *,'blkhark ',blkhark

type *,'blk_hist_path=',blk_hist_path end if !debug

open

1193

open

$1194 \quad 1$ access='append')

$1195 \mathrm{C}$ if the sample is not a blank, read the first set of blank

$1196 \mathrm{C}$ history information into memory location 1

1197

1198

1199

1200

1201

1202

1203

1204

1205

1206

1207

1208

1209

1210

1211

1212

1213

1214

1215

1216

1217

1218

1219

1220

1221

1222

1223

1224

1225

1226

1227

1228

if ( $x$ char ne. 'R') then

nisnum $=1$

read (blkhun, ' $(1 x, a 16,1 x, a 16,1 x, a 20)$ ', end=991) blkbid(nisnum),

1 blkid (nisnum), blkpdat (nisnum)

call str\$upcase (blkbid (nisnum), blkbid (nisnum))

call str\$upcase (blkid (nisnum), blkid (nisnum))

call str\$upcase (blkpdat (nisnum), blkpdat (nisnum))

if (debug(1:1) eq. 'Y') then

type *,' blank history sample no. ',

1 nisnum

type *,' i.d. from history ',

1 blkbid (nisnum), blkid (nisnum)

type *,' process date ',blkpdat (nisnum) end if !debug

do xnumb=1, nbr

c read activity, rbar, and sb for each nuclide

read (blkhun, ' (1x,3g15.8)', end=991)

I nucact (nisnum, xnumb) , rbrblk (nisnum, xnumb),

1 sbeblk (nisnum, xnumb) if (debug $(1: 1)$.eq. 'Y') then type *,' blank activities (muci)',

1 ' for nuclide ', xnumb

type *,' act, rb, sb',

1 nucact (nisnum, xnumb), rbrblk (nisnum, xnumb),

1 sbeblk (nisnum, xnumb)

end do end if ! debug

go to 999

$c$ end of file before finishing first read in blank history

991 continue

blkid $(1)=$ '

blkeof991'

blkbid $(I)=$ '

blkeof991' 
Appendix 2.A Listing of FORTRAN code ALPHA_MDA.FOR file version as of January 10, 1996

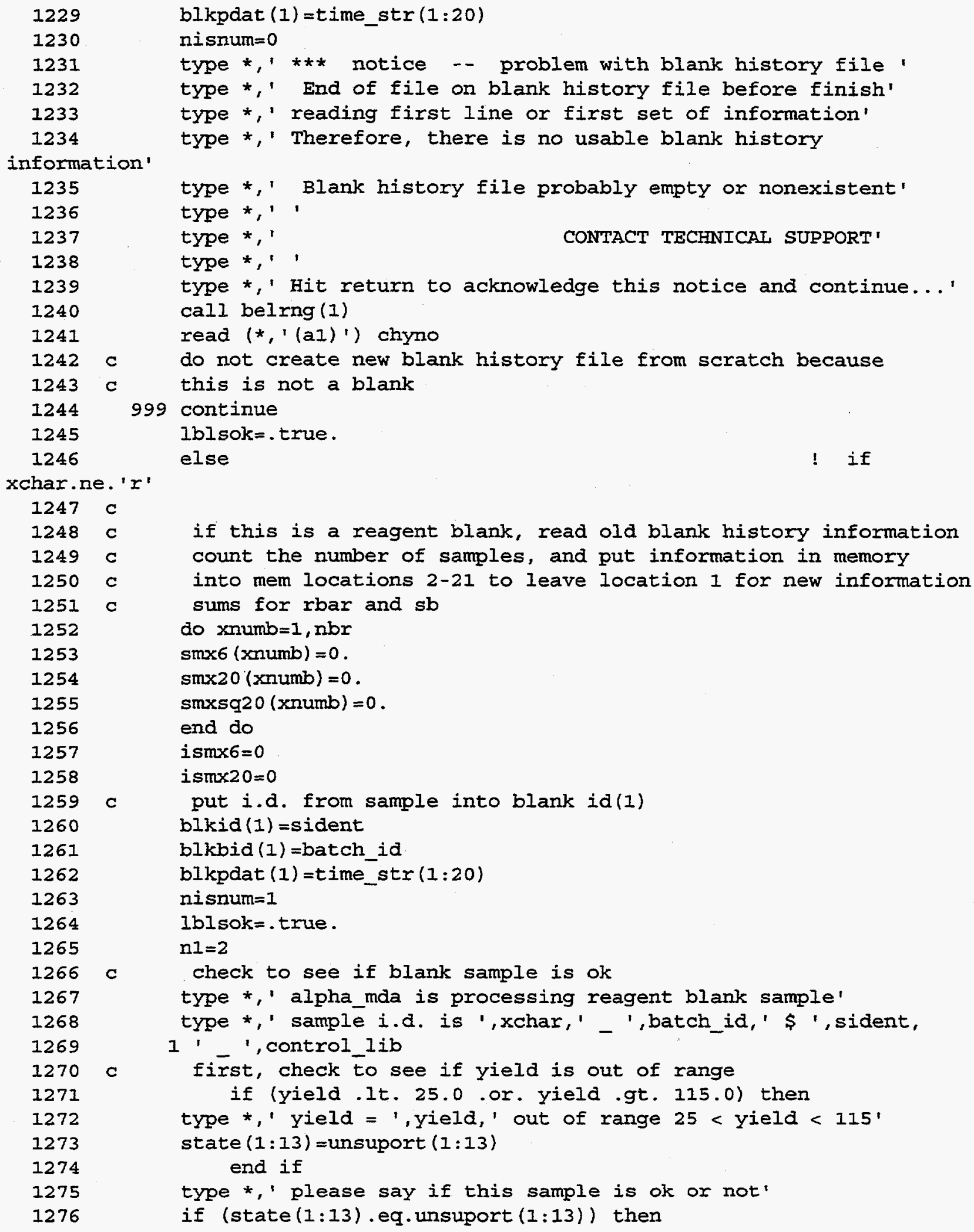


Appendix 2.A Listing of FORTRAN code ALPHA MDA.FOR. file version as of January 10, 1996

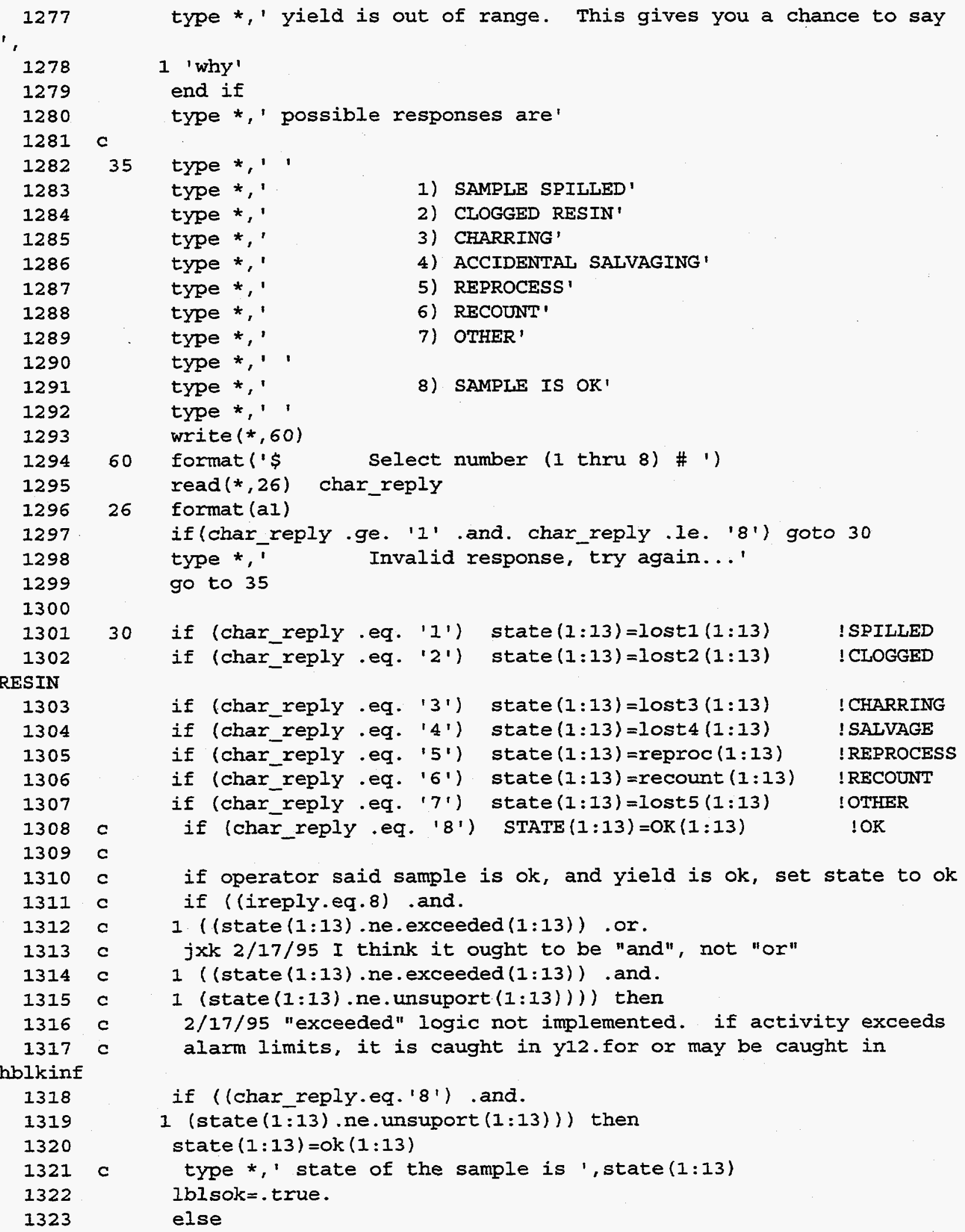




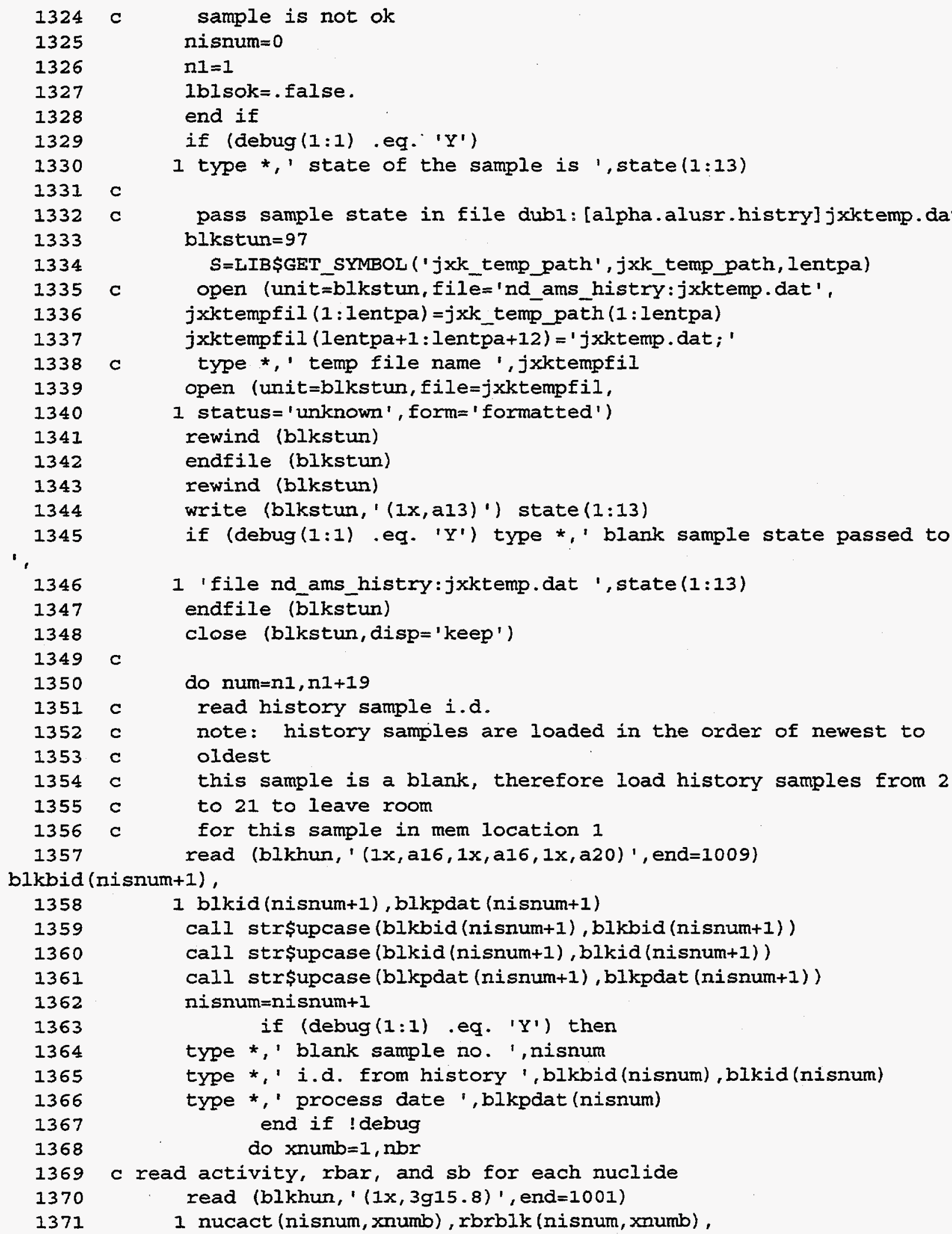


Appendix 2.A Listing of FORTRAN code ALPHA MDA.FOR, file version as of January 10, 1996

1372

1373

1374

1375

1376

1377

1378

1379

1380

1381

1382

1383

1384

1385

1386

1387

1388

1389

1390

1391

1392

1393

1394

1395

1396

1397

1398

1399

1400

xnumb $=1$, nbr

$1401 \mathrm{C}$

duplicate

$1402 \mathrm{C}$

1403

1404

1405

then

1406

1407

1408

1409

1410

1411

1412

1413

1414

1415

1416

num $=2,21$

$1417 \mathrm{C}$
I sbeblk (nisnum, xnumb)

go to 1002

1001 continue

type *,' abnormal end of file on blank history file'

nisnum=nisnum-1

go to 1009

1002 continue

if (debug $(1: 1$ ) .eq. ' $Y$ ') then

type *,' blank activities (muci)',

1 ' for nuclide ', xnumb

type *,' act, rbar, sb ',

1 nucact (nisnum, xnumb), rbrblk (nisnum, xnumb),

1 sbeblk (nisnum, xnumb) end if !debug

c sums for rbar and $s b$

if (ismx6.1t.5) then

smx6 (xnumb) =smx6 (xnumb) +nucact (nisnum, xnumb)

if (xnumb.eq.nbr) ism $x 6=i \operatorname{smx} 6+1$

if (debug $(1: 1)$.eq. ' $Y$ ') then

type *,' record, nuclide, ismx6, smx6'

type *, nisnum, xnumb, ismx 6 , smx6 (xnumb) end if !debug

end if

if (ismx20.1t.19) then

$\operatorname{smx} 20$ ( $x$ numb) $=\operatorname{sm\times 20}$ ( $x$ numb) +nucact (nisnum, $x$ numb)

smxsq20 (xnumb) =smxsq20 (xnumb) + nucact (nisnum, xnumb) $* * 2$

if (xnumb.eq. $n b r$ ) ism $20=$ ism $20+1$

end if

end do

! do

compare current sample i.d. to one just read to check for

don't bother to check if duplicate already found or if nisnum=1 if (nisnum.gt.1 .and.

1 blkid(nisnum).eq.blkid(1)

1 .and. blkbid (nisnum) .eq.blkbid(1) .and. .not.1bldup)

lbldup= . true.

if (debug $(I: I)$.eq. ' $Y$ ') then

type *,' reagent blank sample 1 id:'

type *, blkbid(1), blkid(1)

type *,' duplicates sample ',nisnum,' id:'

type *,blkbid(nisnum), blkid (nisnum) end if ! debug

cjxktestC TEST TEMPORARILY REFUSE TO RECOGNIZE DUPLICATES

cjxktest LBLDUP=.FALSE.

end if

end do

! do 
Appendix 2.A Listing of FORTRAN code ALPHA MDA.FOR, file version as of January 10, 1996

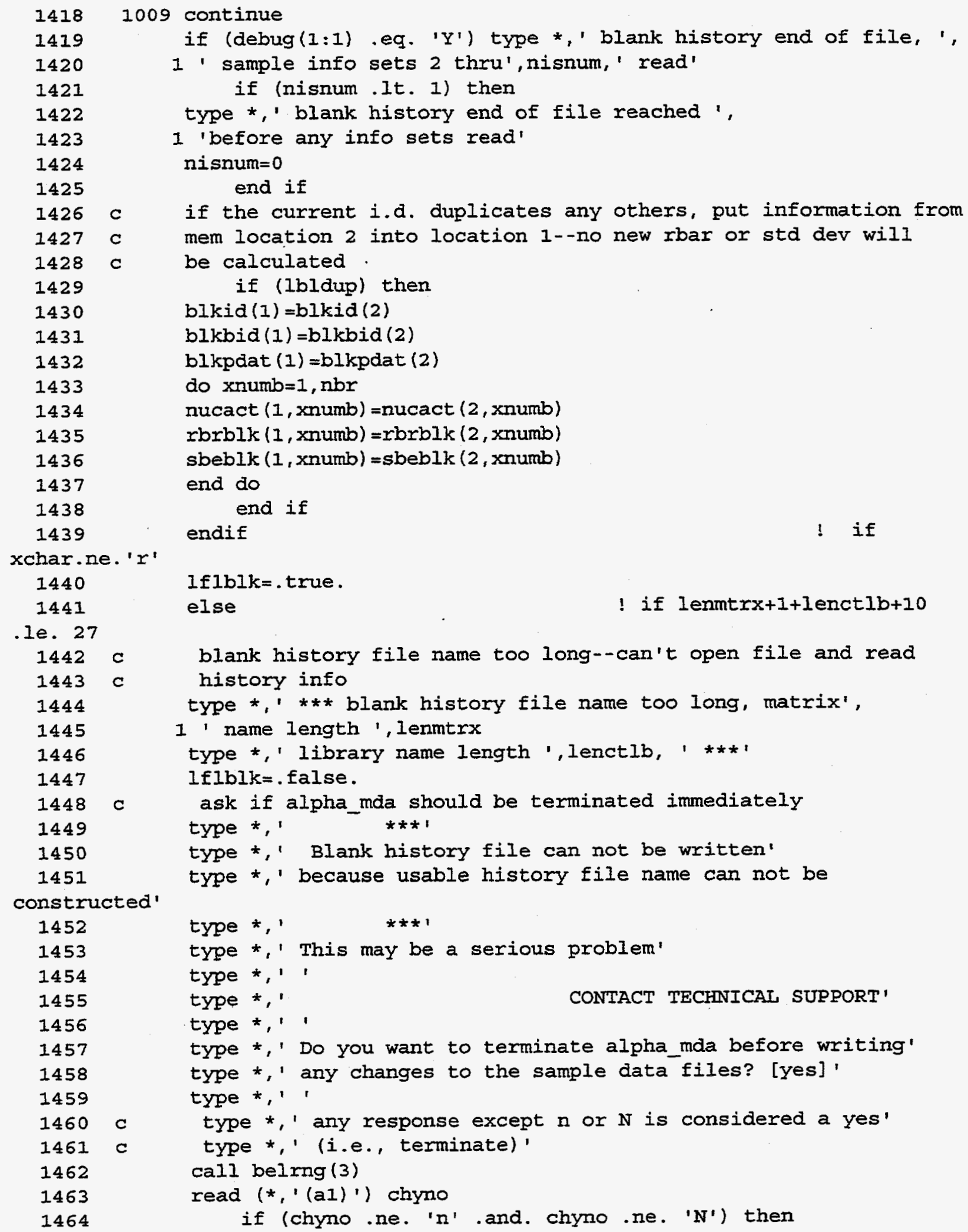


Appendix 2.A Listing of FORTRAN code ALPHA MDA.FOR file version as of January 10, 1996

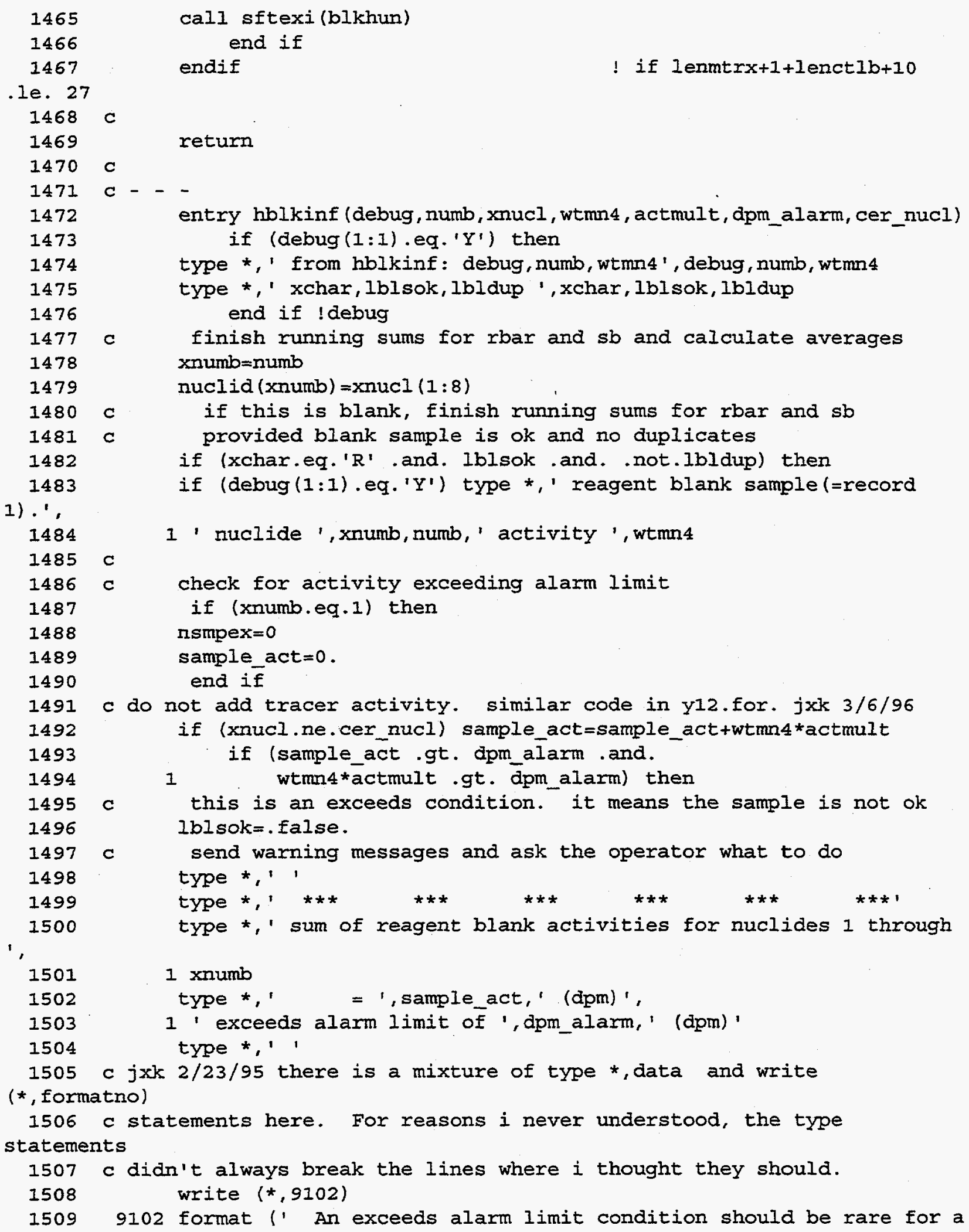

1465

1466

1467

.1e. 27

$1468 \mathrm{C}$

1469

1470

1471

1472

1473

1474

1475

1476

1477

1478

1479

1480

1481

1482

1483

1).',

1484

1485

1486

1487

1488

1489

1490

1491

1492

1493

1494

1495

1496

1497

1498

1499

1500

,

1501

1502

1503

1504

1505

(*, formatno)

1506 c statements here. For reasons $i$ never understood, the type

statements

1507 c didn't always break the lines where $i$ thought they should.

1508 write $(*, 9102)$

15099102 format (' An exceeds alarm limit condition should be rare for a

call sftexi (blkhun)
end if

endif

! if lenmtr $x+1+1$ enct $1 b+10$

return

entry hblkinf (debug, numb, xnucl, wtmn4, actmult, dpm_alarm, cer_nucl)

if (debug $(I: I)$.eq.' $Y$ ') then

type *,' from hblkinf: debug, numb, wtmn4', debug, numb, wtmn4

type *,' xchar, 1blsok, 1bldup ', xchar, 1blsok, Ibldup end if ! debug

finish running sums for rbar and $s b$ and calculate averages xnumb $=$ numb

nuclid (xnumb) =xnucl $(1: 8)$

if this is blank, finish running sums for rbar and sb provided blank sample is ok and no duplicates

if (xchar.eq.' $R$ ' .and. Iblsok .and. not.Ibldup) then

if (debug $(1: 1)$.eq.' 'Y') type *,' reagent blank sample (=record

1 ' nuclide ', xnumb, numb,' activity ', wtmn4

check for activity exceeding alarm limit

if (xnumb.eq.1) then

nsmpex $=0$

sample_act $=0$.

end $i \bar{f}$

$c$ do not add tracer activity. similar code in y12. for. jxk $3 / 6 / 96$

if (xnucl.ne.cer_nucl) sample_act=sample_act+wtmn4*actmult

if (sample_act.gt. apm_alarm .and.

1 wtmn 4 actmult.gt. dpm_alarm) then

this is an exceeds condition. It means the sample is not ok

lblsok= . false.

send warning messages and ask the operator what to do

type *,' '

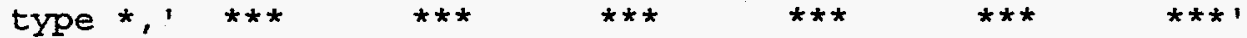

type *,' sum of reagent blank activities for nuclides 1 through

1 xnumb

type *,' = ', sample_act,' (dpm)',

1 'exceeds alarm limit of ', dpm_alarm,' (dpm)' type *,' '

c jxk 2/23/95 there is a mixture of type *, data and write

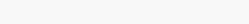


Appendix 2.A Listing of FORTRAN code ALPHA MDA.FOR, file version as of January 10, 1996

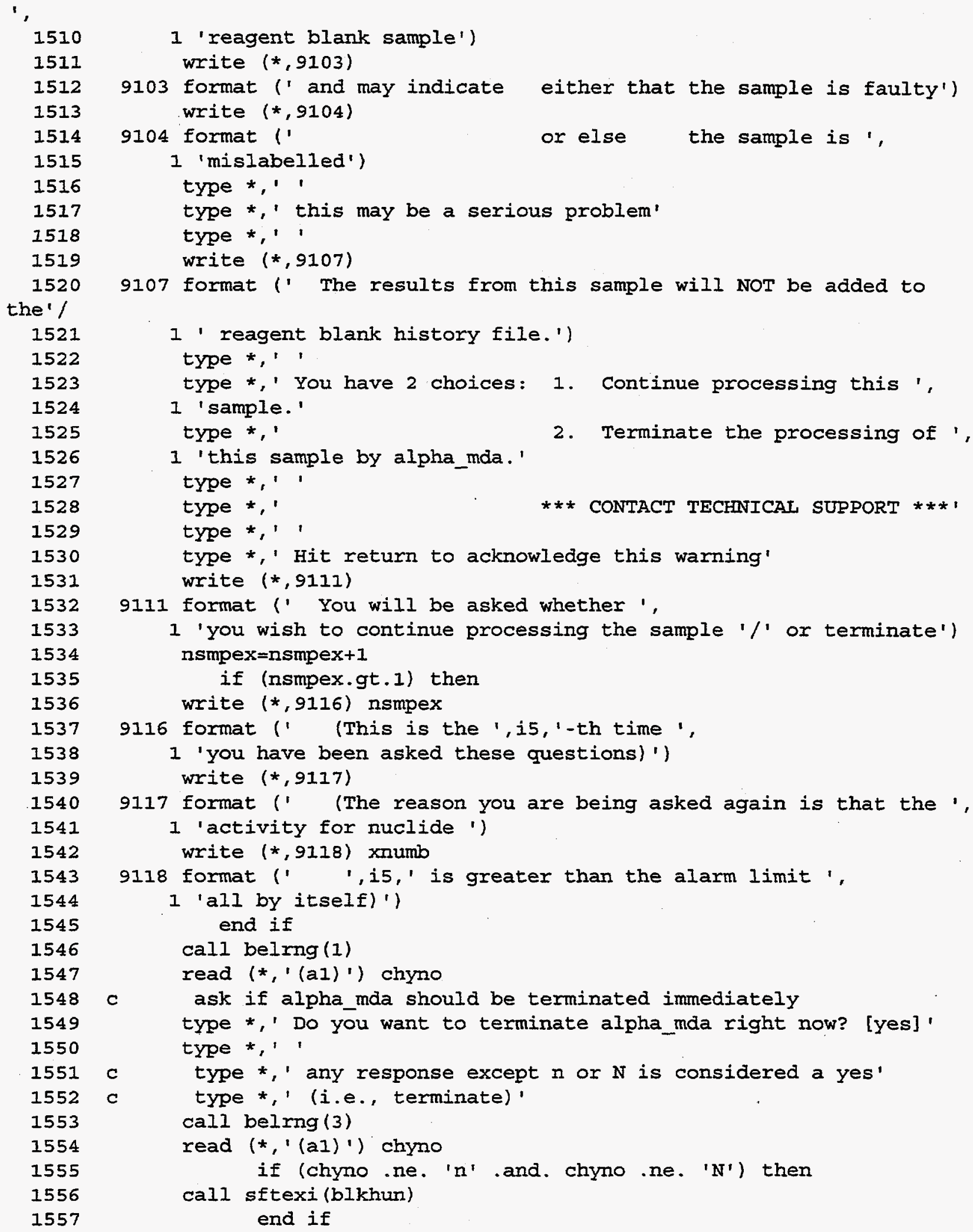

1510

1511

1512

1513

1514

1515

1516

1517

1518

1519

1520

the'/

1521

1522

1523

1524

1525

1526

1527

1528

1529

1530

1531

1532

1533

1534

1535

1536

1537

1538

1539

1540

1541

1542

1543

1544

1545

1546

1547

1548

1549

1550

1551

1552

1553

1554

1555

1556

1557 
Appendix 2.A Listing of FORTRAN code ALPHA MDA.FOR, file version as of January 10, 1996

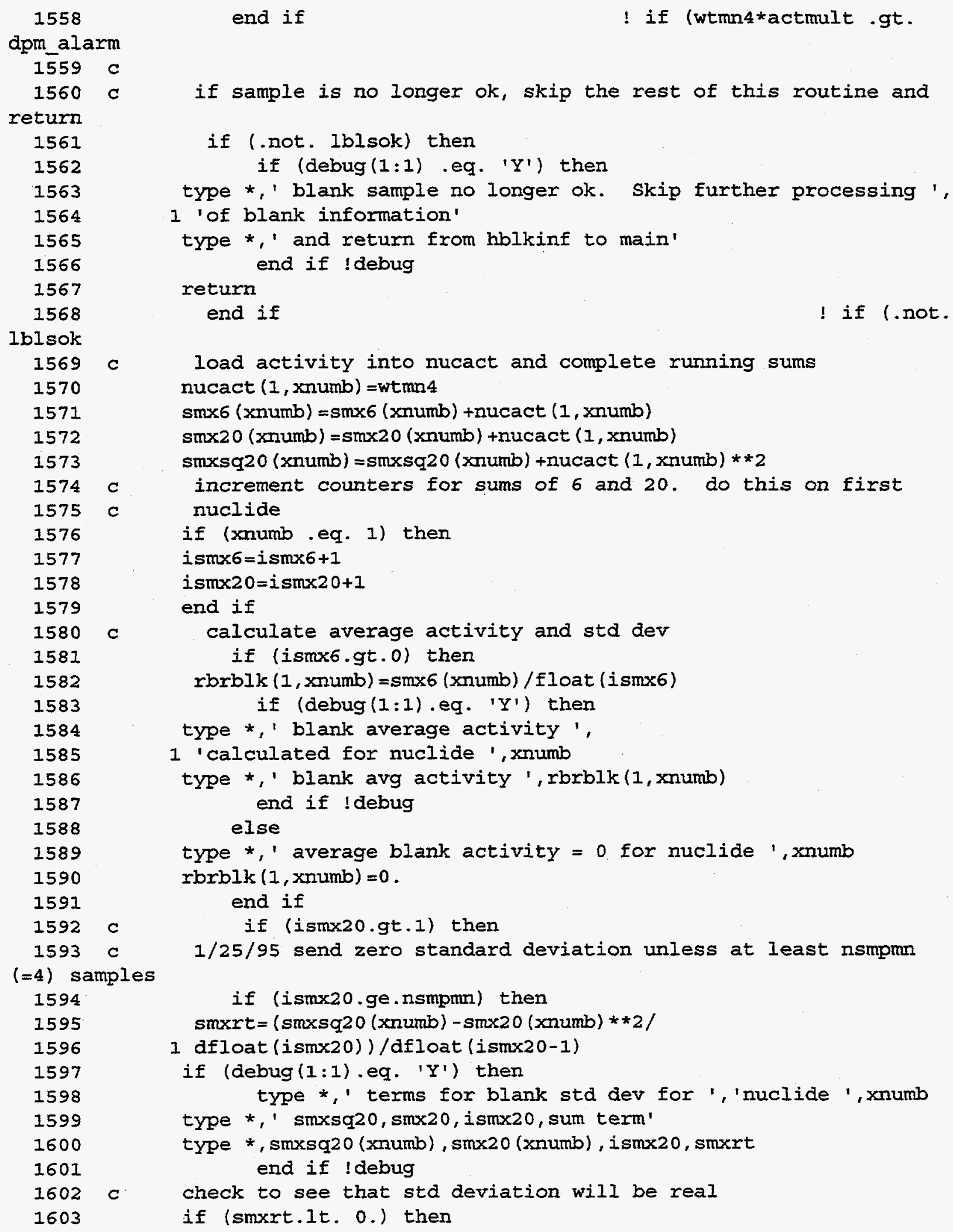


Appendix 2.A Listing of FORTRAN code ALPHA MDA.FOR, file version as of January 10, 1996

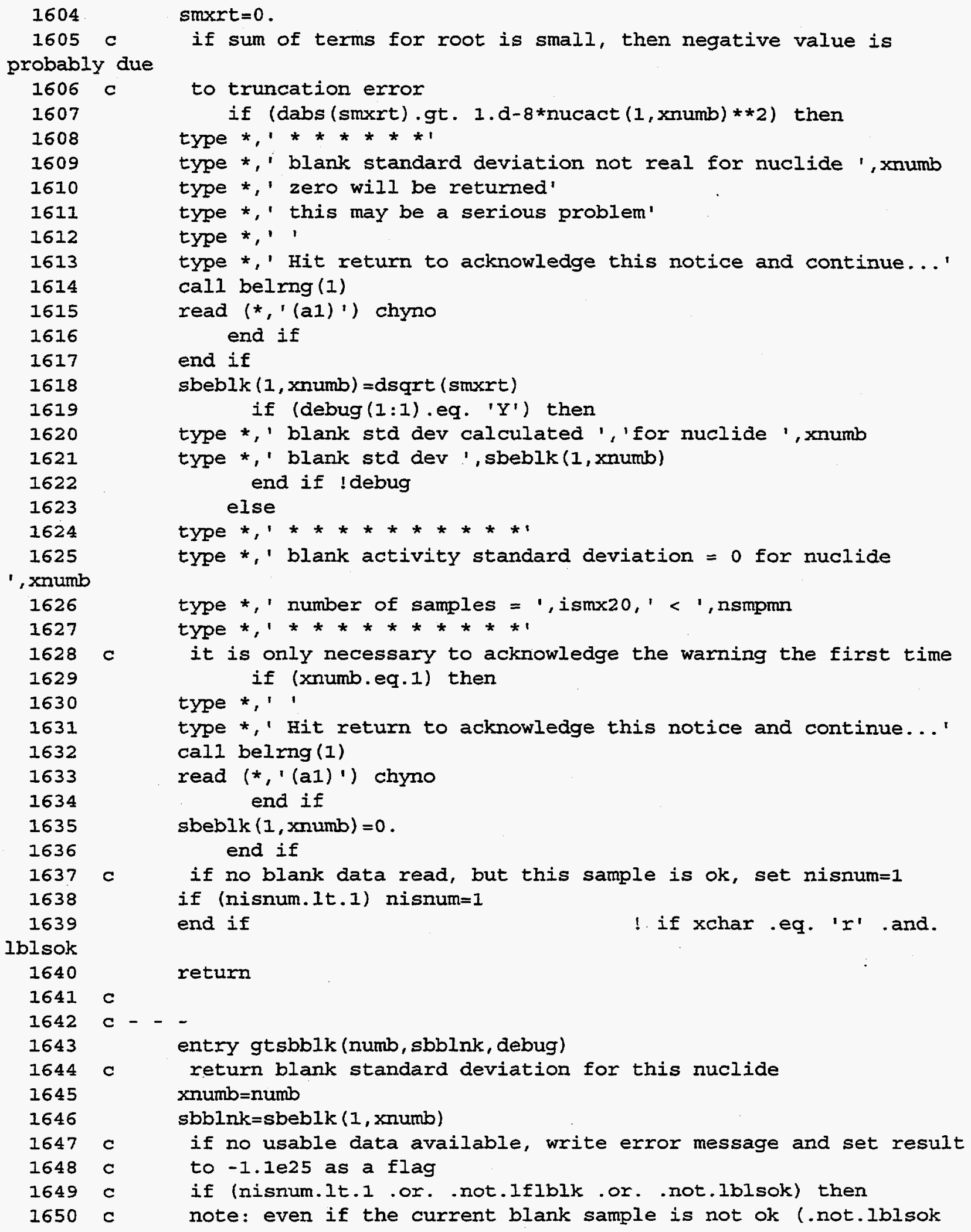


Appendix 2.A Listing of FORTRAN code ALPHA MDA.FOR, file version as of January 10, 1996

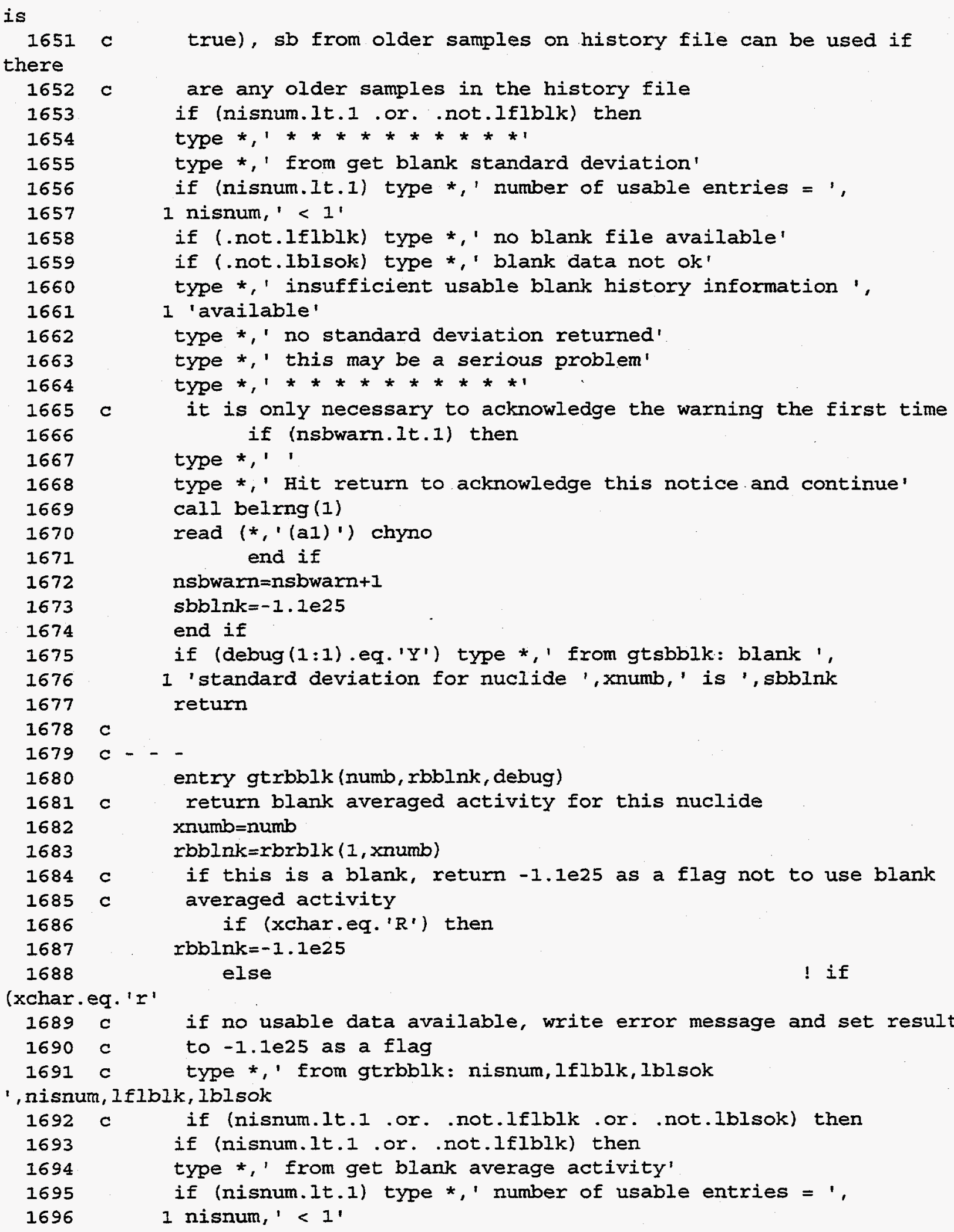




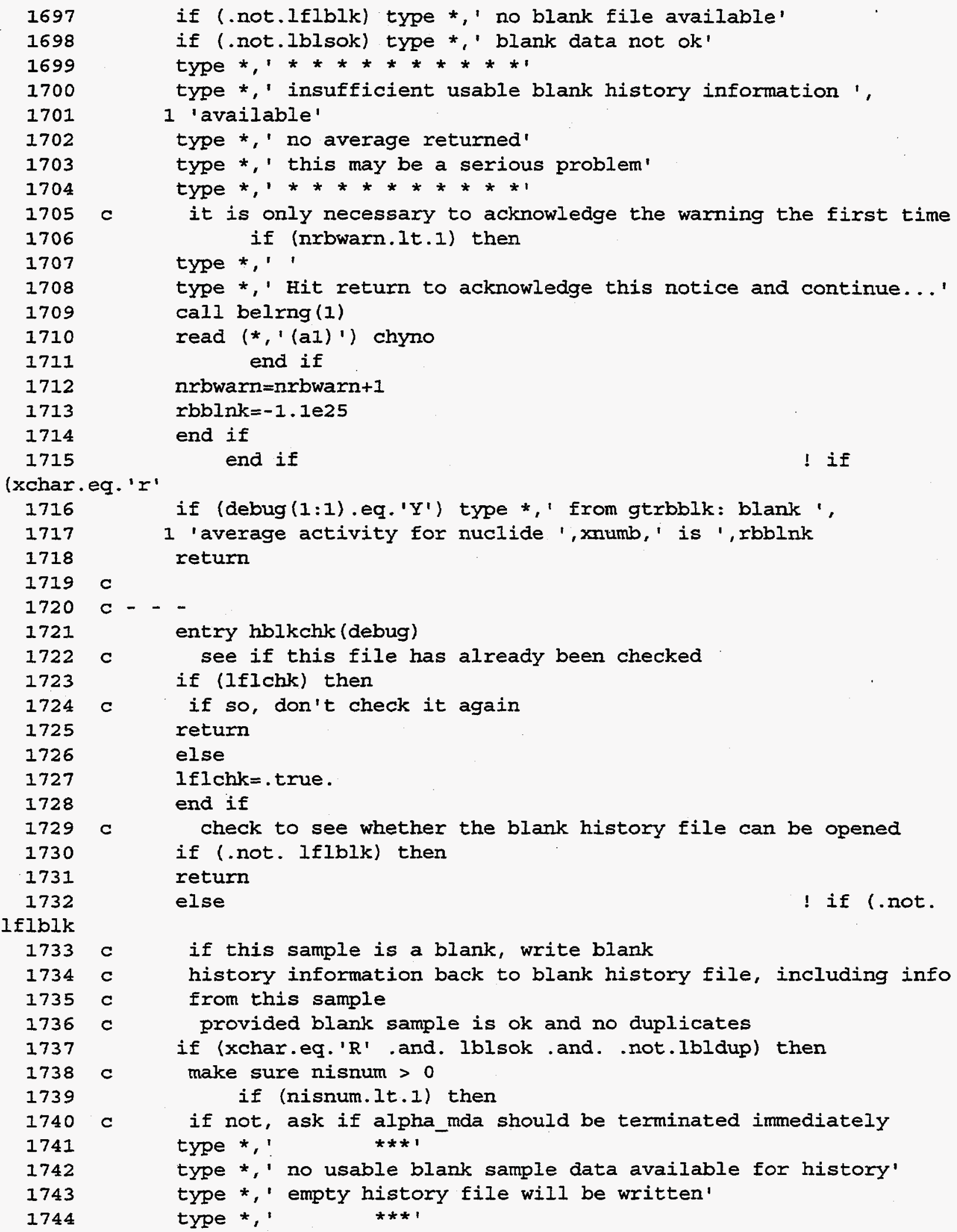




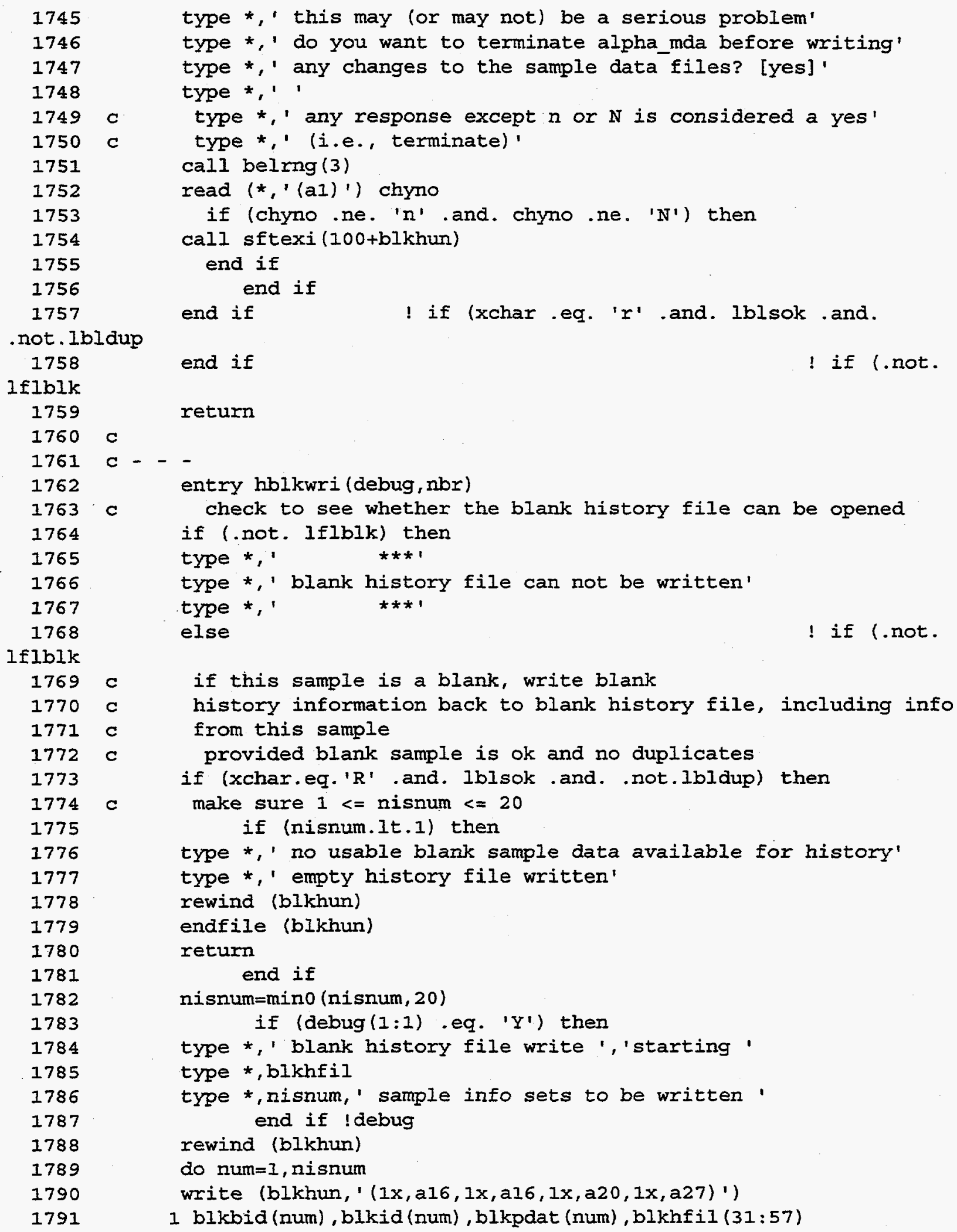

1745

1746

1747

1748

1749

1750

1751

1752

1753

1754

1755

1756

1757

. not. Ibldup

1758

Iflblk

1759

1760

1761

1762

1763

1764

1765

1766

1767

1768

Iflblk

1769

1770

1771

1772

1773

1774

1775

1776

1777

1778

1779

1780

1781

1782

1783

1784

1785

1786

1787

1788

1789

1790

1791

type *,' this may (or may not) be a serious problem' type *,' do you want to terminate alpha maa before writing' type *,' any changes to the sample data files? [yes]' type *,' '

type *,' any response except $n$ or $N$ is considered a yes'

type *,' (i.e., terminate)'

call belrng (3)

read (*,' (aI)') chyno

if (chyno .ne. ' $n$ ' and. chyno .ne. 'N') then

call sftexi (100+blkhun)

end if

$$
\text { end if }
$$

end if

! if (xchar .eq. ' $r$ ' and. Iblsok . and.

end if

! if (.not.

return

c

c - - -

c

entry hblkwri (debug, nbr)

check to see whether the blank history file can be opened if (.not. Iflblk) then

type *,

type *,' blank history file can not be written'

type *,'

$\star * * 1$

else

! if (.not.

C if this sample is a blank, write blank

c history information back to blank history file, including info

from this sample

provided blank sample is ok and no duplicates

if (xchar.eq.' $R$ ' .and. Iblsok .and. not.lbldup) then

make sure $1<=$ nisnum $<=20$

if (nisnum.1t.1) then

type *,' no usable blank sample data available for history'

type *,' empty history file written'

rewind (blkhun)

endfile (blkhun)

return

end if

nisnum=mino (nisnum, 20)

if (debug $(I: I)$.eq. 'Y') then

type *,' blank history file write ','starting '

type *,blkhfil

type *, nisnum,' sample info sets to be written ' end if !debug

rewind (blkhun)

do num $=1$, nisnum

write (blkhun, ' (Ix, a16, Ix, a16, Ix, a20, 1x, a27) ')

1 blkbid (num), blkid (num) , blkpdat (num) , blkhfil (31:57) 
Appendix 2.A Listing of FORTRAN code ALPHA MDA.FOR, file version as of January 10, 1996

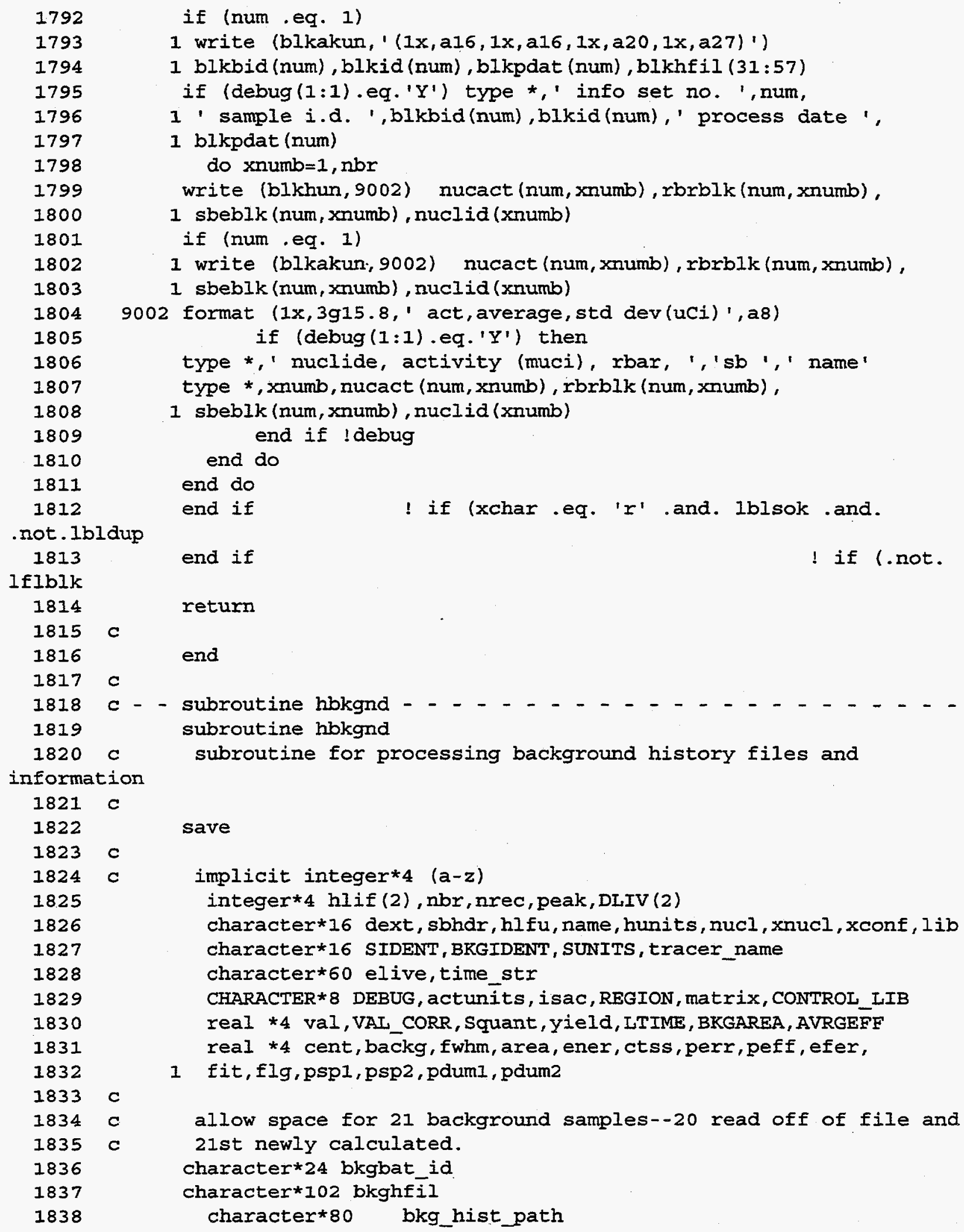




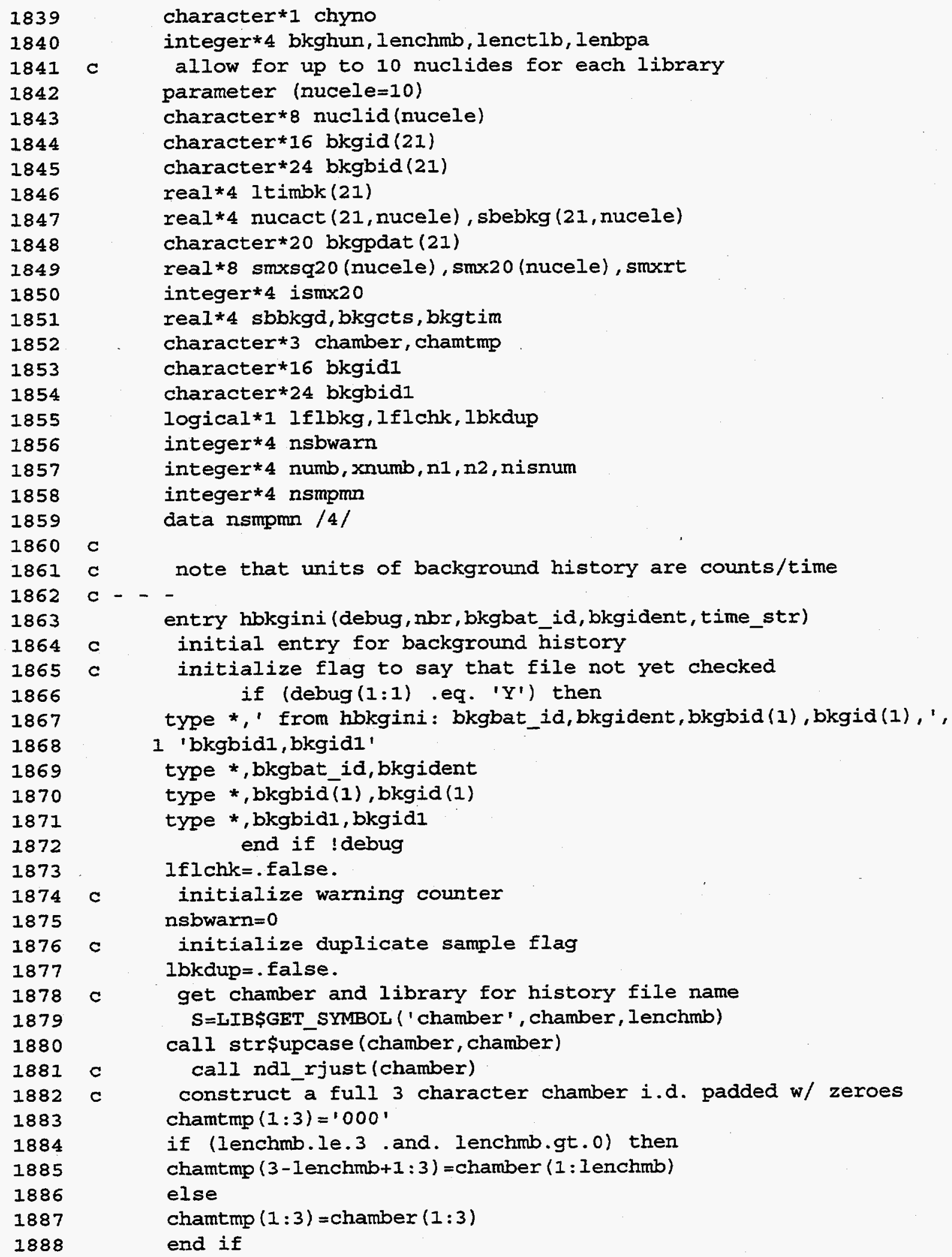


Appendix 2.A Listing of FORTRAN code ALPHA MDA.FOR, file version as of January 10.1996

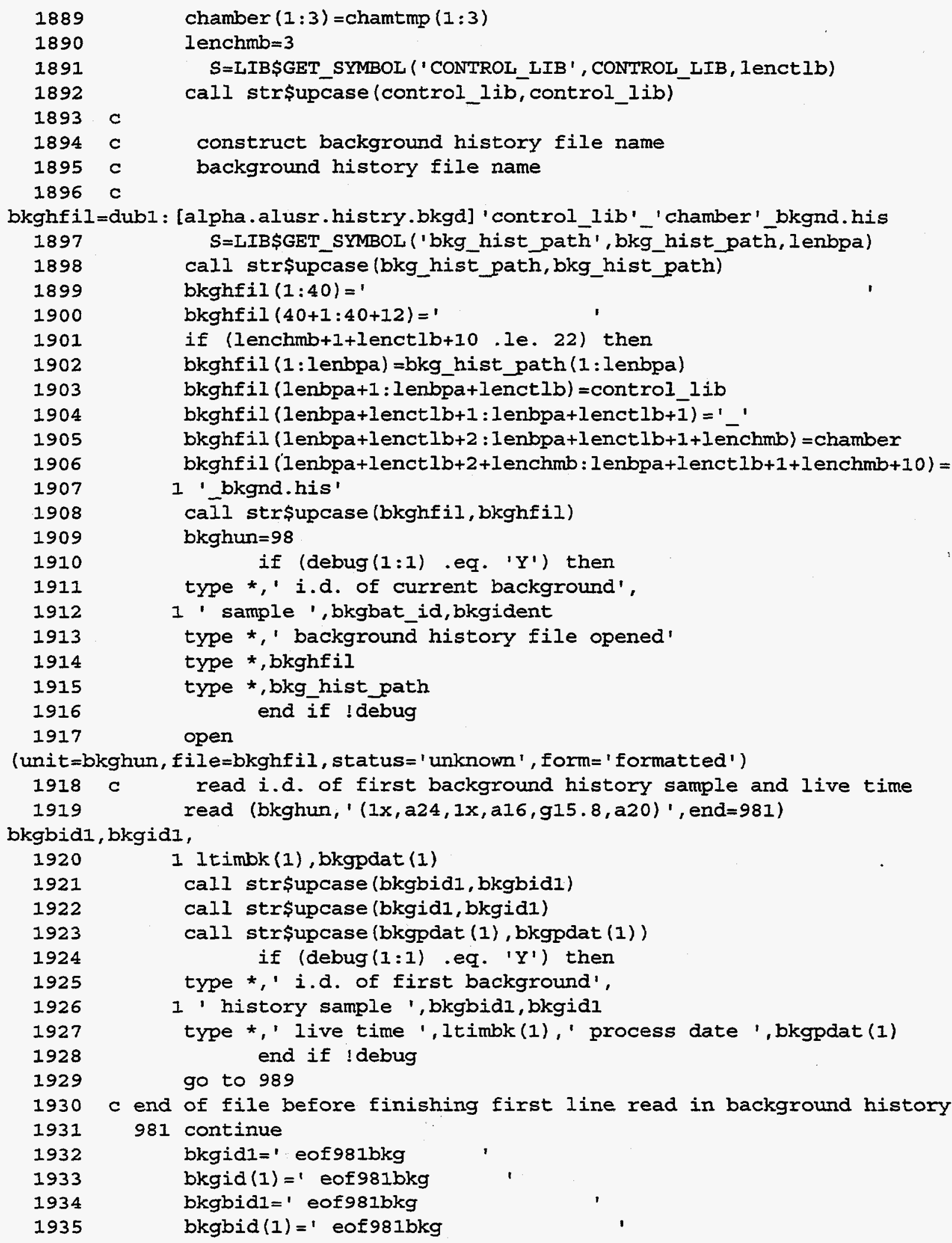


Appendix 2.A Listing of FORTRAN code ALPHA MDA.FOR file version as of January 10, 1996

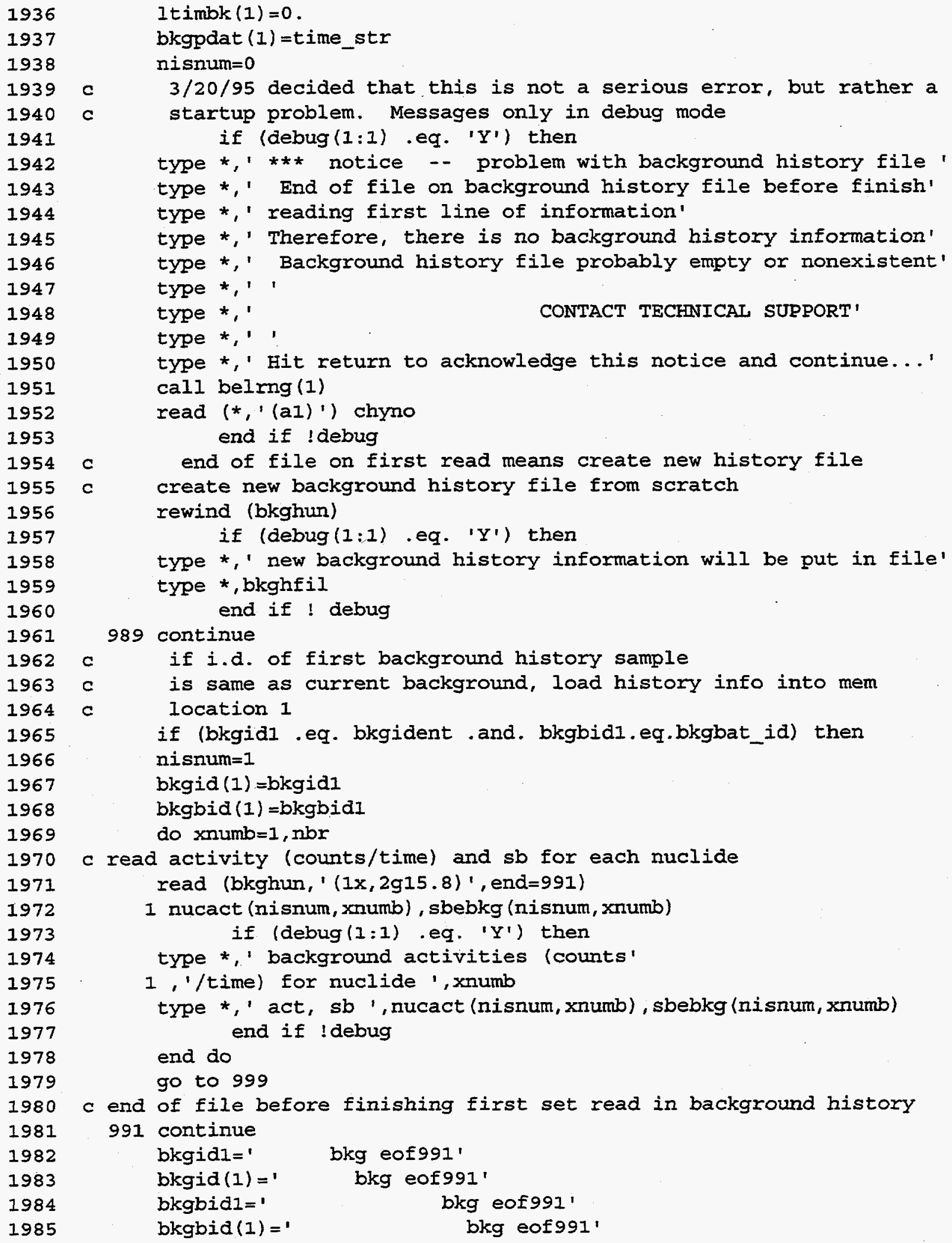




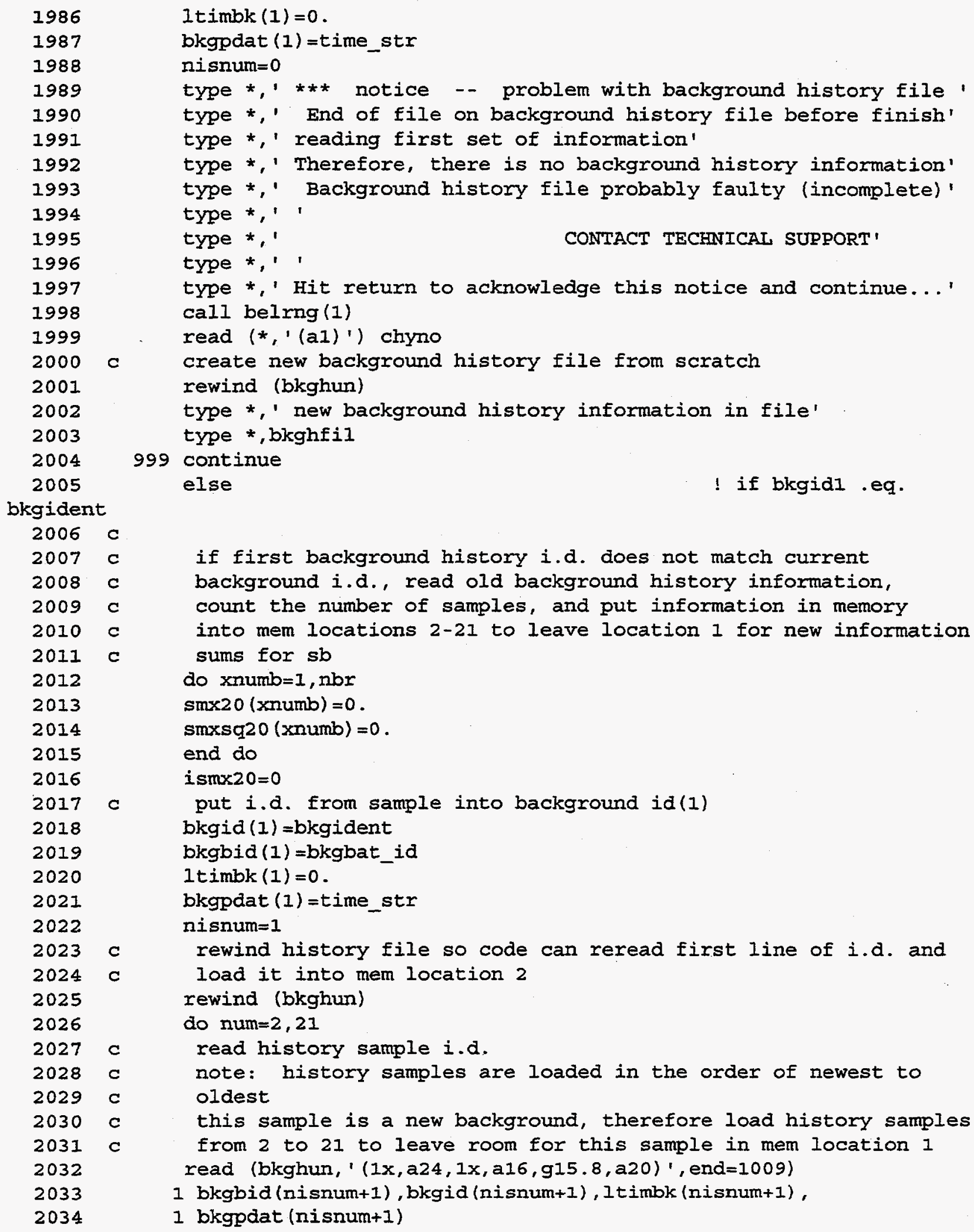




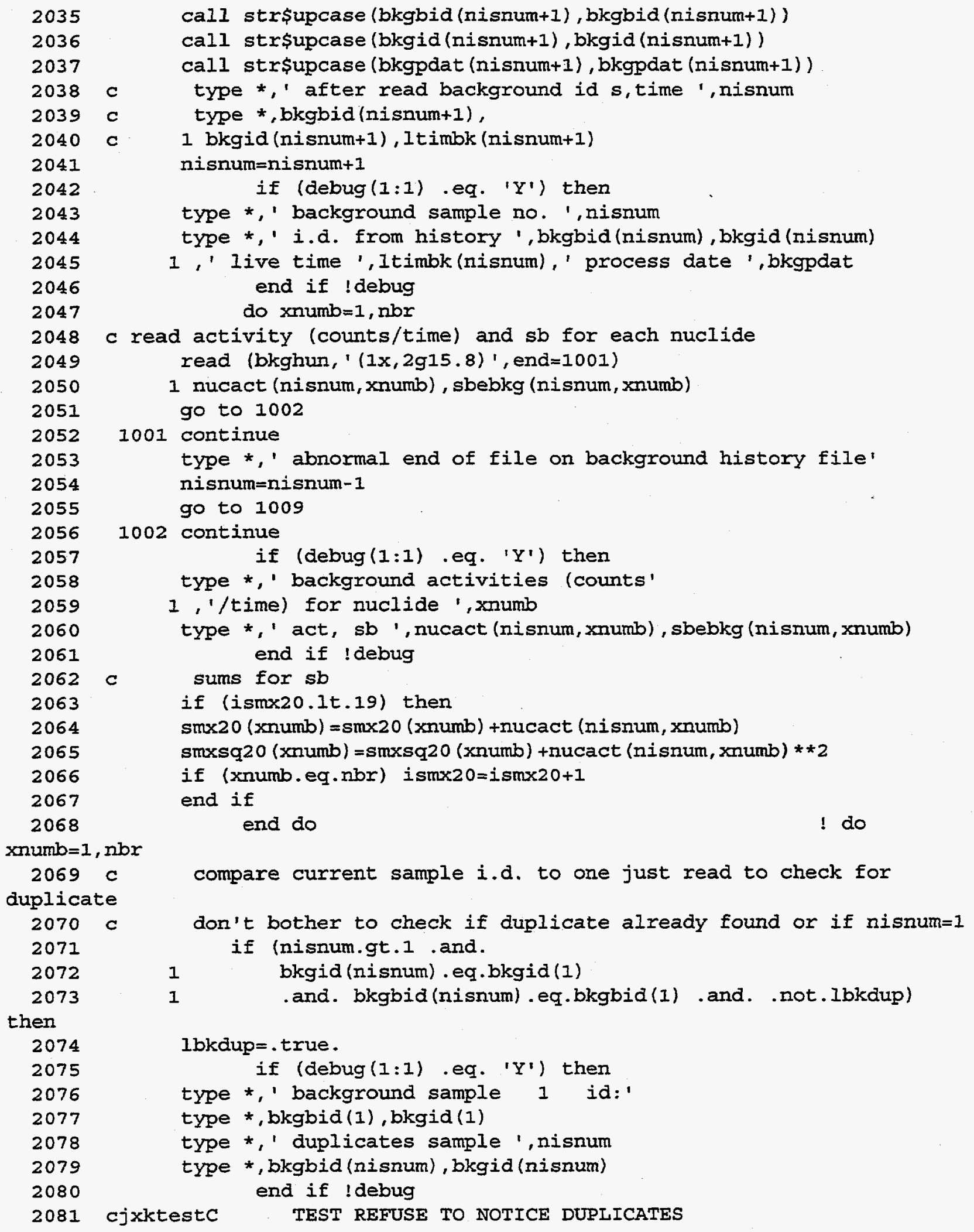


Appendix 2.A Listing of FORTRAN code ALPHA MDA.FOR, file version as of January 10, 1996

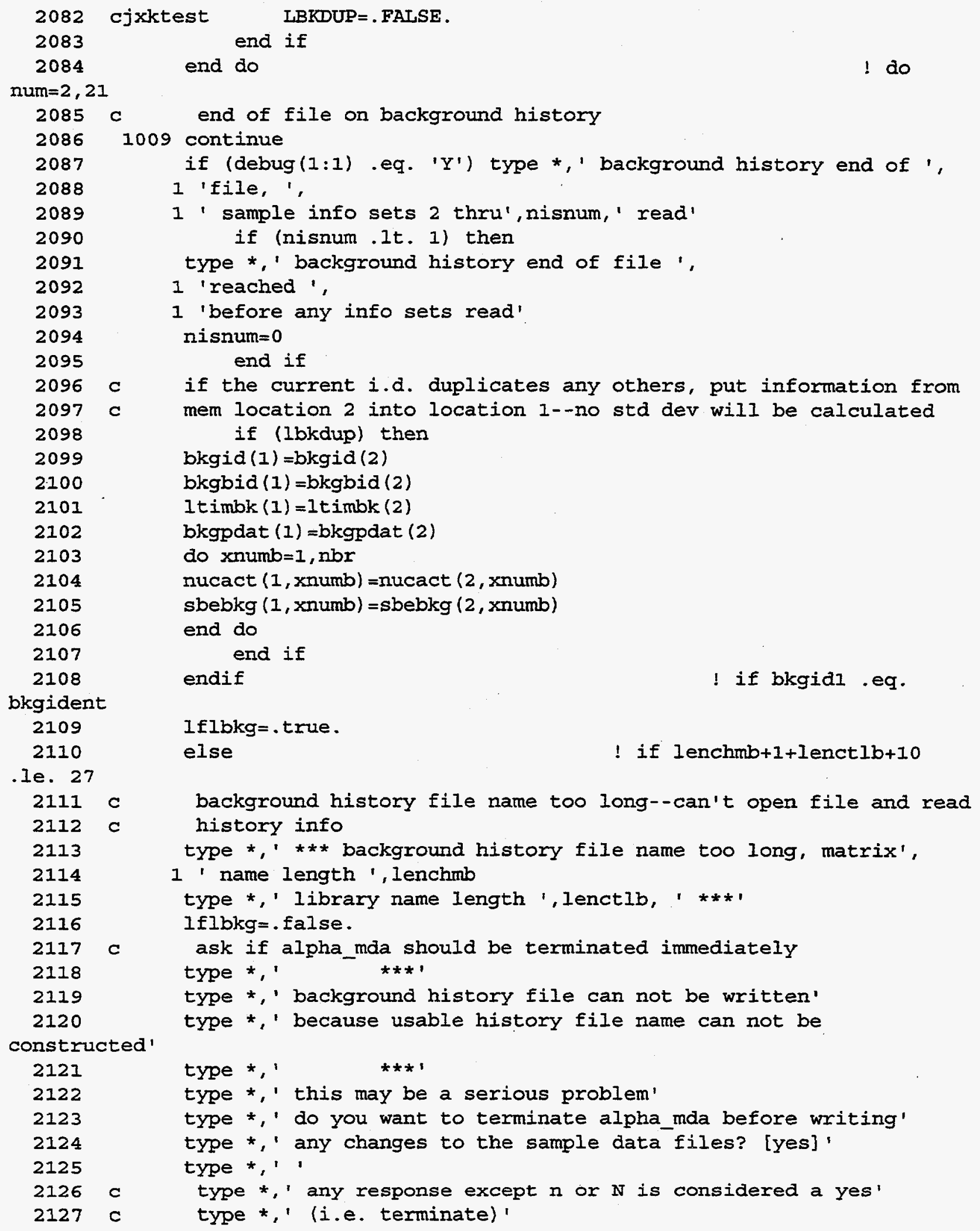


2128

2129

2130

2131

2132

2133

.1 e. 27

2134

2135

2136

2137

2138

2139

2140

2141

2142

2143

2144

2145

2146

2147

2148

2149

2150

2151

2152

2153

',

2154

2155

2156

2157

2158

2159

2160

2161

2162

2163

2164

2165

2166

nsmpmn

2167

2168

2169

2170

2171

2172

2173

2174 call belrng(3)

read (*,'(a1)') chyno

if (chyno .ne. ' $n$ ' .and. chyno .ne. ' $N$ ') then

call sftexi (bkghun) end if

endif

! if lenchmb $+1+$ lenct $l b+10$ c

C c - -

entry hbkginf (debug, numb, xnucl, bkgcts, bkgtim,bkgbat_id,bkgident)

C finish running sums for $\mathrm{sb}$ and calculate standard deviation xnumb $=$ numb nuclid (xnumb) =xmucl $(1: 8)$ if (debug $(1: 1)$.eq. 'Y') then type *,' from hbkginf: bkgbat_id,bkgident,bkgbid(1),bkgid(1),' ,

1 'bkgbid1, bkgid1, lbkdup' type *, bkgbat_id, bkgident type *, bkgbia(1), bkgid(1) type *, bkgbidl, bkgid1, lbkdup end if ! debug if background $i . d$. does not match first line read from history file and no other duplicates, finish running sums for sb if ((bkgidl.ne.bkgident .or. bkgbidl.ne.bkgbat_id)

1 .and. not. Ibkdup) then if (debug( $1: 1$ ) .eq. 'Y') type *,' background sample (=record 1 ).

1 ' nuclide ', xnumb,' counts/sec ',bkgcts,' time ', bkgtim nucact $(1$, xnumb) $=$ bkgcts

Itimbk (I) =bkgtim $\operatorname{smx} 20$ (xnumb) =smx20 (xnumb) +nucact $(1$, xnumb)

smxsq20 (xnumb) =smxsq20 (xnumb) +nucact $(1$, xnumb) **2

c increment counter for sum of 20 . do this on first

c nuclide

if (xnumb .eq. 1) then

ism $\times 20=i \operatorname{sm} \times 20+1$

end if

calculate std dev

if (ismx20.gt.I) then

$1 / 12 / 95$ decision made that standard deviation for fewer than

c

C

(=4) samples not worthwhile, so make special provisions if (ismx20.ge.nsmpmn) then $\operatorname{smxrt}=(\operatorname{smxsq20}(\mathrm{xnumb})-\operatorname{smx} 20(\mathrm{xnumb}) * * 2 /$

1 dfloat (ismx20))/dfloat (ismx20-1) if (debug $(1: 1)$.eq. 'Y') then

type *,' terms for backgr std dev for ', 'nuclide ', xnumb type *,' smxsq20, smx20, ismx20, sum term' type *, smxsq20 (xnumb), smx20 (xnumb), ismx20, smxrt 
Appendix 2.A Listing of FORTRAN code ALPHA MDA.FOR, file version as of January 10, 1996

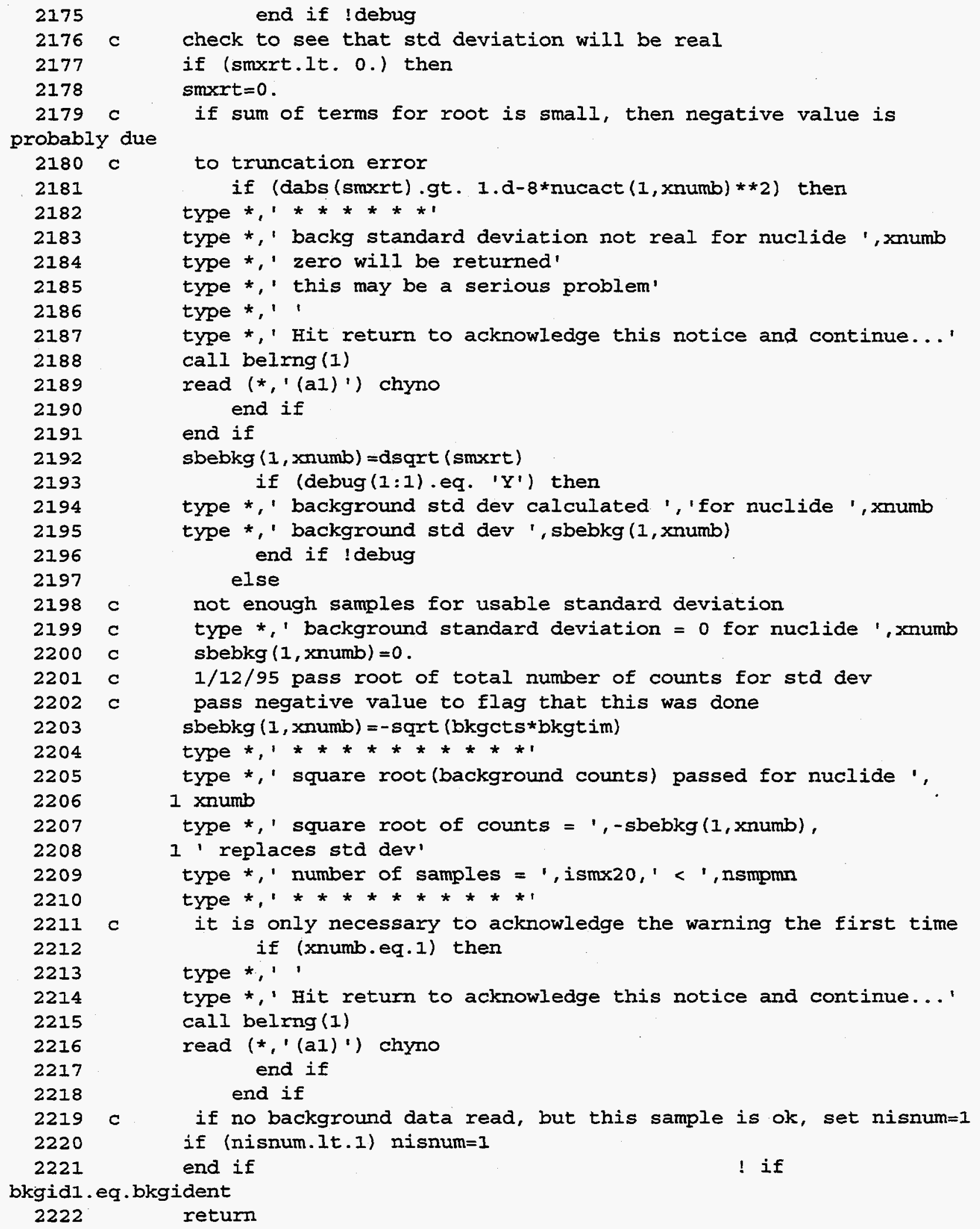




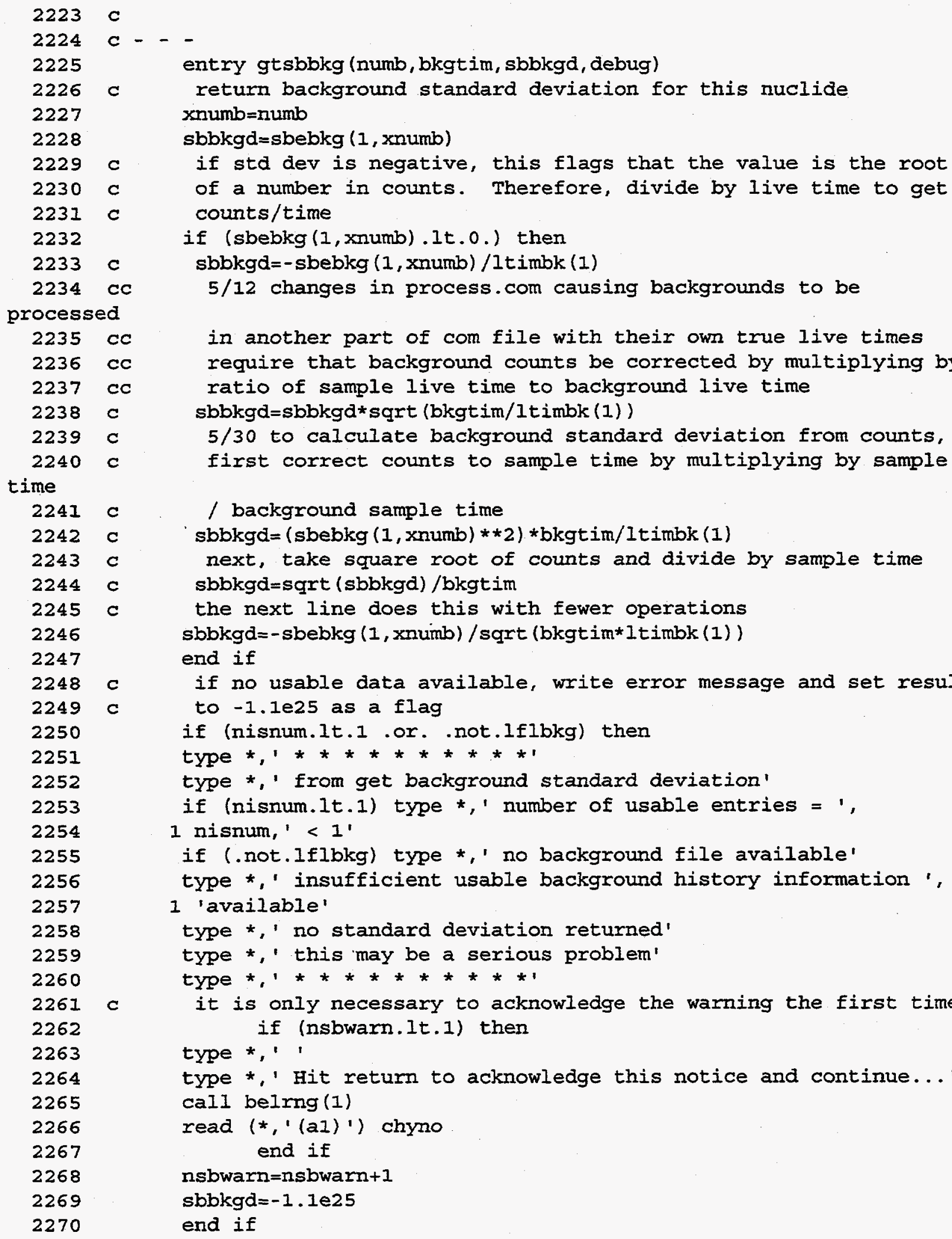


Appendix 2.A Listing of FORTRAN code ALPHA_MDA.FOR, file version as of January 10, 1996

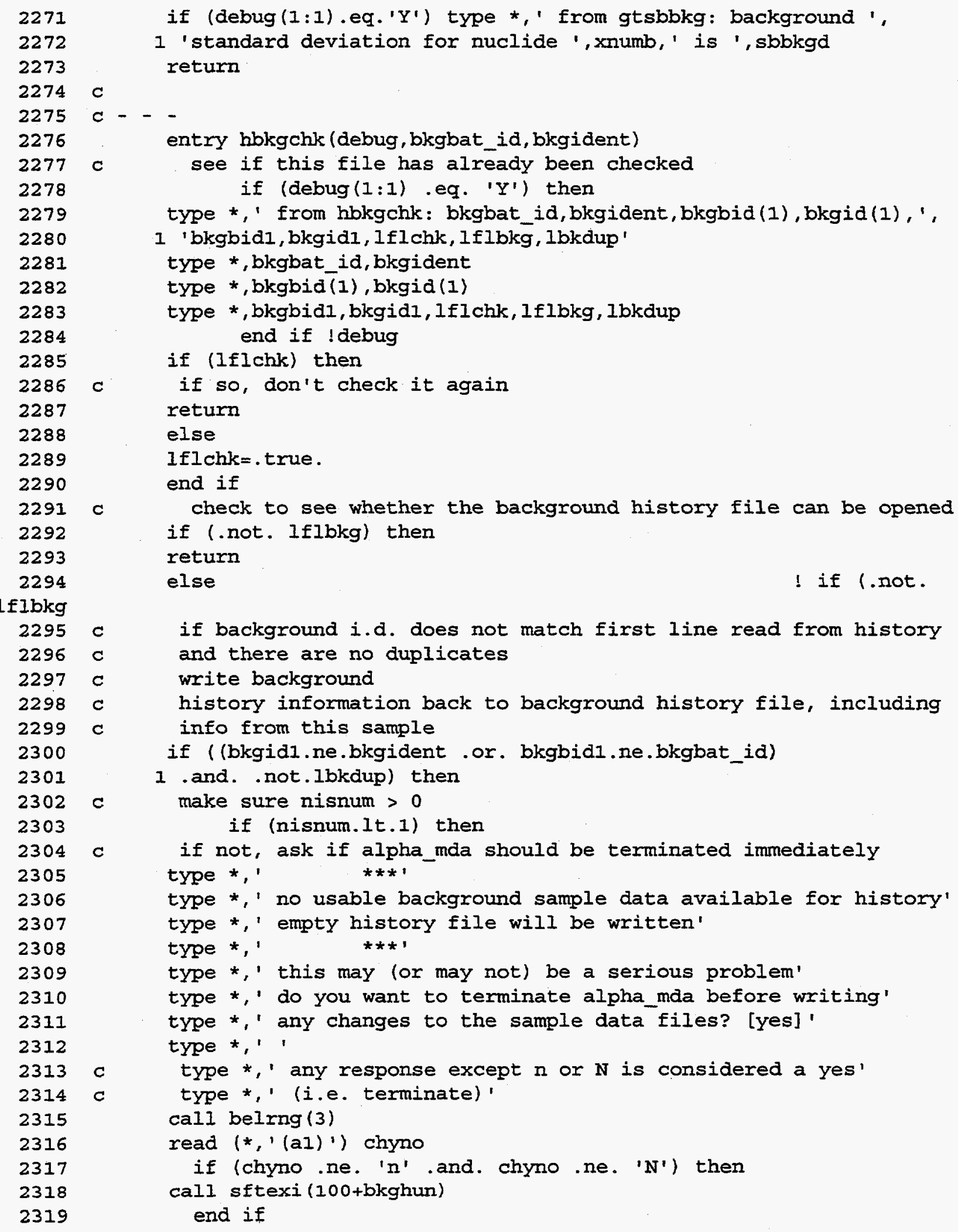


Appendix 2.A Listing of FORTRAN code ALPHA MDA.FOR. file version as of January 10, 1996

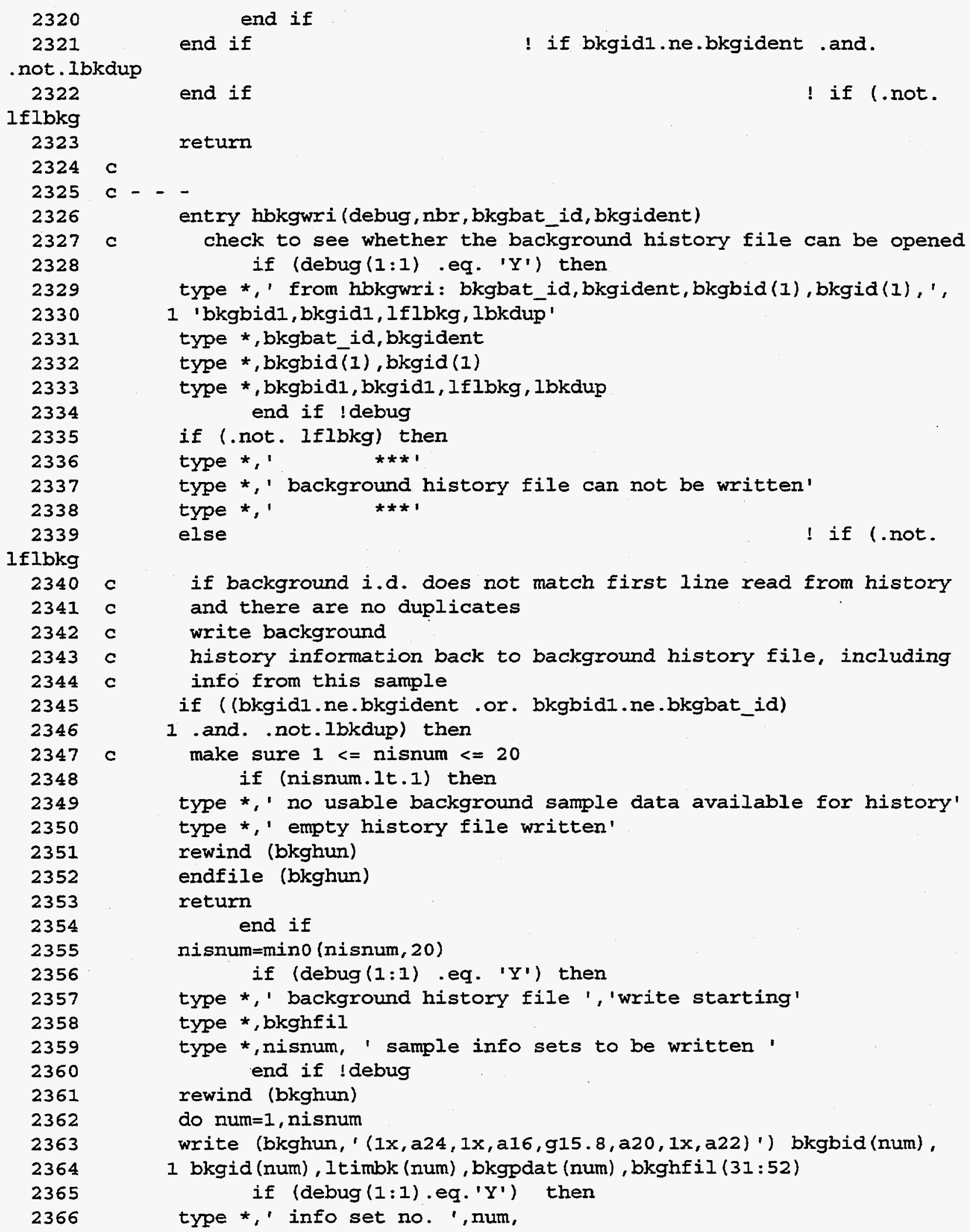

2320

2321

. not. Ibkdup

2322

$1 f l b k g$

2323

2324

2325

2326

2327

2328

2329

2330

2331

2332

2333

2334

2335

2336

2337

2338

2339

$1 \mathrm{flbkg}$

2340

$2341 \mathrm{C}$

$2342 \mathrm{C}$

$2343 \mathrm{C}$

$2344 \mathrm{C}$

2345

2346

2347

2348

2349

2350

2351

2352

2353

2354

2355

2356

2357

2358

2359

2360

2361

2362

2363

2364

2365

2366

$$
\text { end if }
$$

end if

end if

! if bkgidl.ne.bkgident .and.

! if (.not. 
Appendix 2.A Listing of FORTRAN code ALPHA MDA.FOR, file version as of January 10, 1996

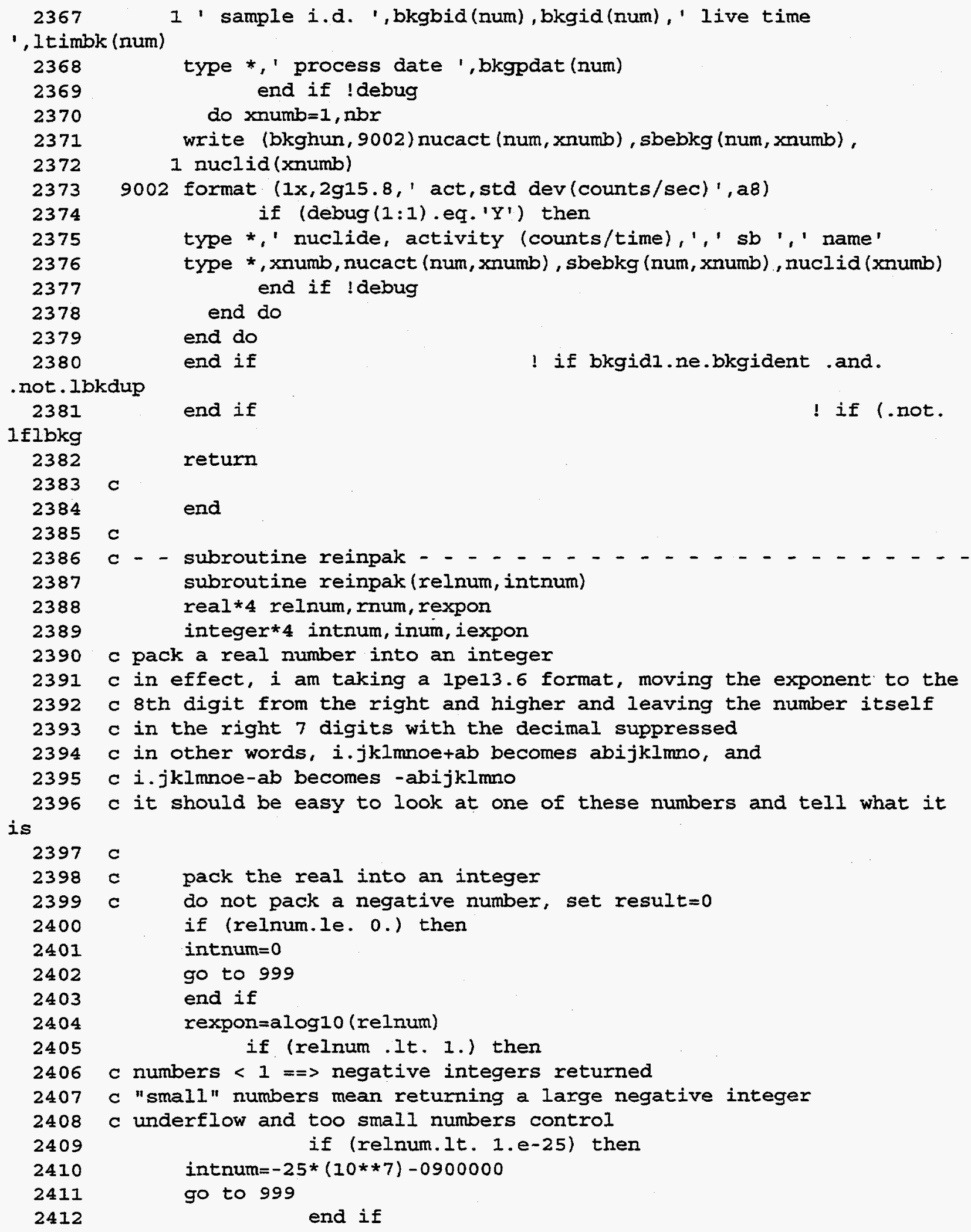


Appendix 2.A Listing of FORTRAN code ALPHA MDA.FOR, file version as of January 10, 1996

is

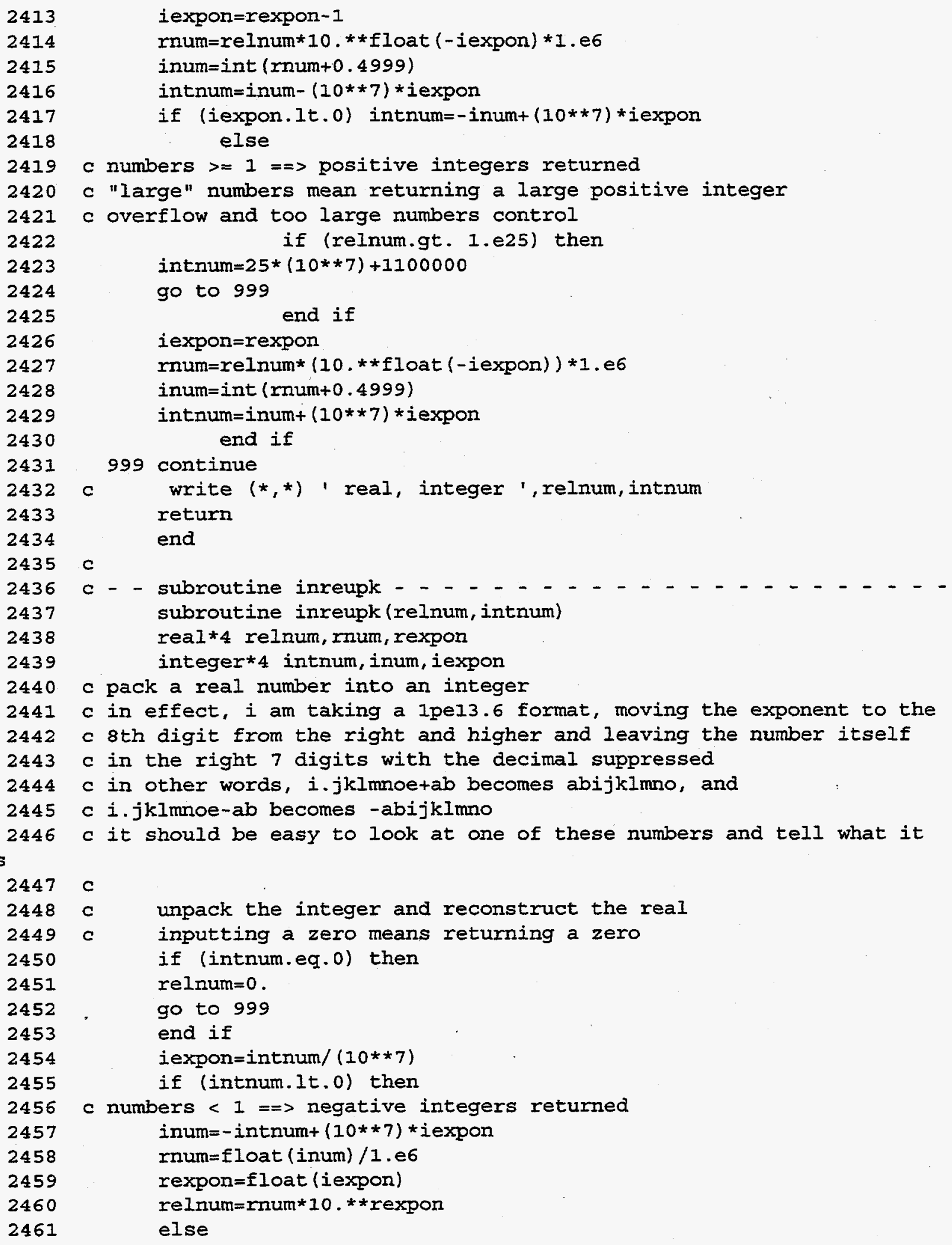

2413

2414

2415

2416

2417

2418

2419

2420

2421

2422

2423

2424

2425

2426

2427

2428

2429

2430

2431

2432

2433

2434

2435

2436

2437

2438

2439

2440

2441

2442

2443

2444

2445

2446

2447

2448

2449

2450

2451

2452

2453

2454

2455

2456

2457

2458

2459

2460

2461

i expon $=$ rexpon -1

rnum $=$ relnum*10.**float $(-$ iexpon $) * I . e 6$

inum=int (rnum+0.4999)

intnum $=$ inum $-(10 * * 7) *$ iexpon

if (iexpon.1t.0) intnum $=-$ inum $+(10 * * 7) *$ iexpon else

c numbers $>=1==>$ positive integers returned

c "large" numbers mean returning a large positive integer

$c$ overflow and too large numbers control

if (relnum.gt. 1.e25) then

intnum $=25 *(10 * * 7)+1100000$

go to 999

end if

iexpon=rexpon

rnum $=$ relnum* $(10 . * *$ float $(-$ iexpon $)) * 1 . e 6$

inum $=$ int (rnum+0.4999)

intnum $=$ inum $+(10 * * 7) *$ iexpon

end if

999 continue

c write $(*, *)$ ' real, integer ', relnum, intnum

return

end

c

c - subroutine inreupk - - - - - - - - - - - - - - - - subroutine inreupk (relnum, intnum)

real*4 relnum, rnum, rexpon

integer*4 intnum, inum, iexpon

c pack a real number into an integer

$c$ in effect, $i$ am taking a 1pel3.6 format, moving the exponent to the

$c$ sth digit from the right and higher and leaving the number itself

$c$ in the right 7 digits with the decimal suppressed

$c$ in other words, $i . j k l m n o e+a b$ becomes abijklmno, and

c $i . j k l m n o e-a b$ becomes -abijklmno

$c$ it should be easy to look at one of these numbers and tell what it

c

c unpack the integer and reconstruct the real

c inputting a zero means returning a zero

if (intnum.eq.0) then

relnum $=0$.

go to 999

end if

iexpon=intnum/ $(10 * * 7)$

if (intnum.1t.0) then

c numbers $<1=\Rightarrow$ negative integers returned

inum $=-$ intnum $+(10 * * 7) *$ iexpon

rnum $=$ float (inum) $/ 1 . e 6$

rexpon=float (iexpon)

relnum $=$ rnum*10.** rexpon

else 
Appendix 2.A Listing of FORTRAN code ALPHA MDA.FOR, file version as of January 10, 1996

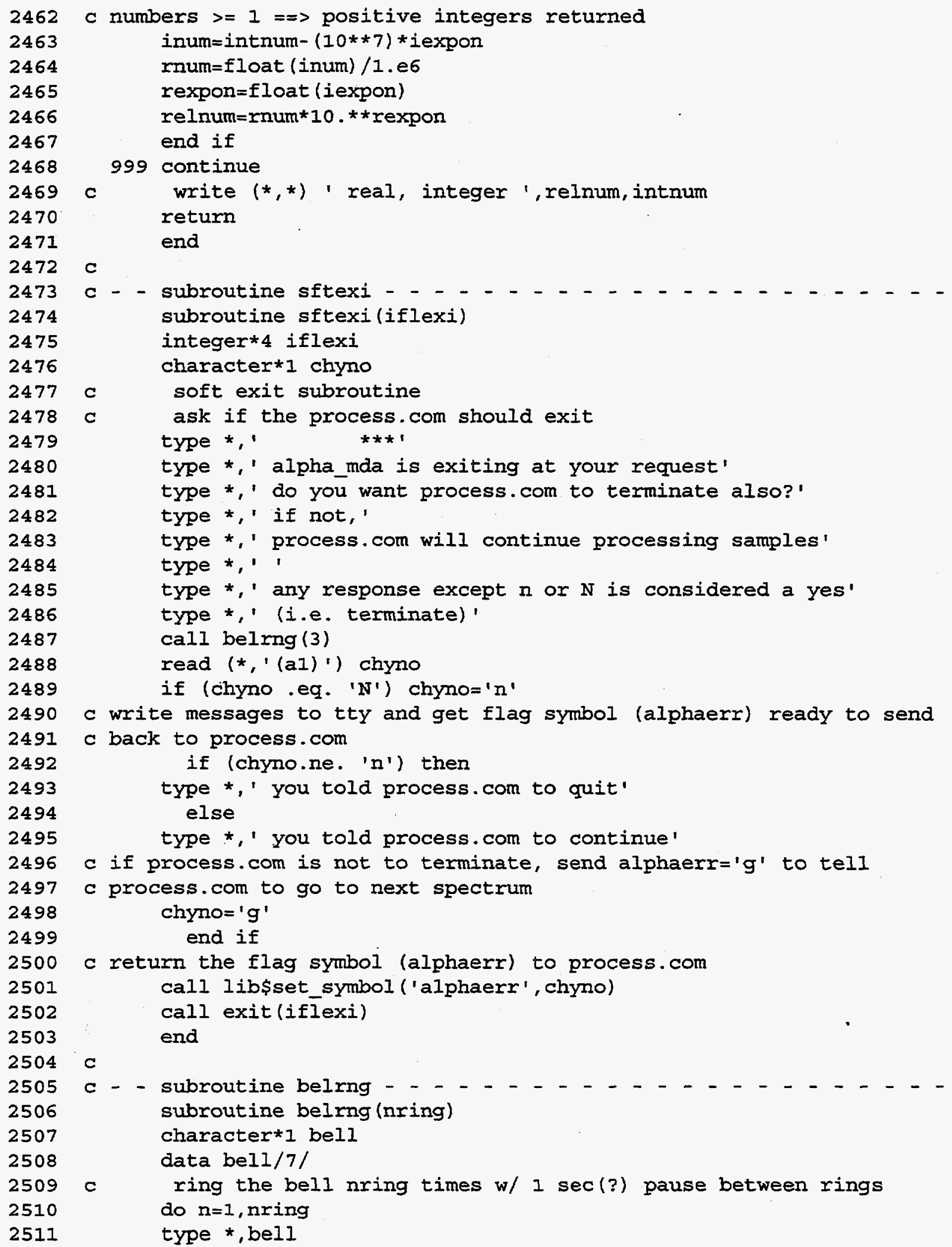


Appendix 2.A Listing of FORTRAN code ALPHA MDA.FOR, file version as of January 10, 1996

2512

2513

2514

2515

$2516 c$

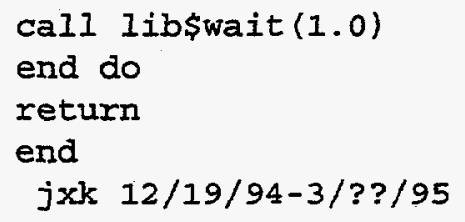




\section{APPENDIX 2.B LISTING OF DCL FILE NDAL_PROCESS.COM, FILE VERSION AS OF JANUARY 10, 1996}

File is located on system B disk at dub0:[000000.nd_root.ndamscust]

Tue Jan 9 08:56:10 EST 1996

$\$$ cat $-\mathrm{n}$ proceskc.com

$1 \$$

2 \$ goto start !(ndal_PROCESS.COM)

3

4 Jan 92 This is the processing for all of the configurations

5 listed in names.dat.

6

7 May 92 Updated with BS's primary/secondary calibration sources

8 for weekly cals.

9

$105 / 18 / 92$ Added code for new flags to control hard copy printing.

11 This is only ok if there is an output device. There is a special

12 flag for each report type. E.g., nid_hc, list_hc etc. They all

13 end in _hc.

14

15 The report_queue is "' if there is not printer queue!

16

17

18

19

20

added code to control printing for:

- wtmean

- channel list

- enback

- sample peak/region

- backgrounds peak/region

- nid

- post nid

not done for:

- control

(Flag names:)

- mda

control hc

- calibration summary calsum_he

$307 / 2 / 92$ Adjustments for 9/2/92 added code for plotting spectrum plot_hc

31 1/5/93 Code to do move/fpl on prime/second processing

32 4/7/92 Added code for post nid peak search postnid hc

33

$345 / 93 \mathrm{dvm}$ it corporation

35 added code for batch processing

36

$375 / 93 \mathrm{dvm}$ it corporation

38 changed to use it's plot program

39

$405 / 93 \mathrm{dvm}$ it corporation 
41

42

43

$445 / 93$

57

58 \$ start:

$59 \$$

$60 \$$

$61 \$$

$62 \$$

$63 \$$

$64 \$$

$65 \$$

$66 \$$ !

$67 \$$ ! setup names and qualifiers for temporary results files

$68 \$$

$69 \$$

$70 \$$

$71 \$$

$72 \$$

$73 \$$

$74 \$$

$75 \$$

$76 \$$

$77 \$$

$78 \$$

$79 \$$

$80 \$$

$81 \$$

$82 \$$

$83 \$$

$84 \$$

$85 \$ !$

$86 \$$

changed to use /compressed form and headers for channel by

channel listing.

dvm it corporation

changed post-nid peak search to run only when report asked for.

dvm it corporation

cleaned up printouts to print in order, and to use headers, and to delete temporary files after printing.

\section{dvm it corporation}

re-installed overall background processing, peak search and channel by channel listings to background printouts.

$$
\text { background spectra. }
$$

\section{on control_y then goto abort_exit}

on error then goto abort_exit

if debug then set verify

if not. debug then set noverify

flat_file_output $=$ f $\$$ edit(flat_file_output, "upcase")

$$
\text { plot_line }=\text { "/list="plot_file'" }
$$$$
\text { plot_file = "nd_ams_list:plot.lis;" }
$$

if dvm

then

$$
\text { chan1_file = "nd_ams_list:chan1.lis;" }
$$

chan1_line $=$ "/list="chan1_file"

chan2 file = "nd ams list:chan2.lis;"

chan2_line $=$ "/list $="$ chan2_file"

else

list_file = "nd_ams_list:list.lis;"

list_line = "/list="list_file""

endif !dvm

gross_file = "nd_ams_list:gross.lis;"

gross_line $=$ "/list $=$ "gross_file""

bkgnd_file = "nd_ams_list:bkgnd.lis;"

bkgnd_line $=$ "/list="b̄kgnd_file""

background_lis = "nd_ams_list:background.lis;"

enback_file = "nd_ams_list:enback.lis;" 


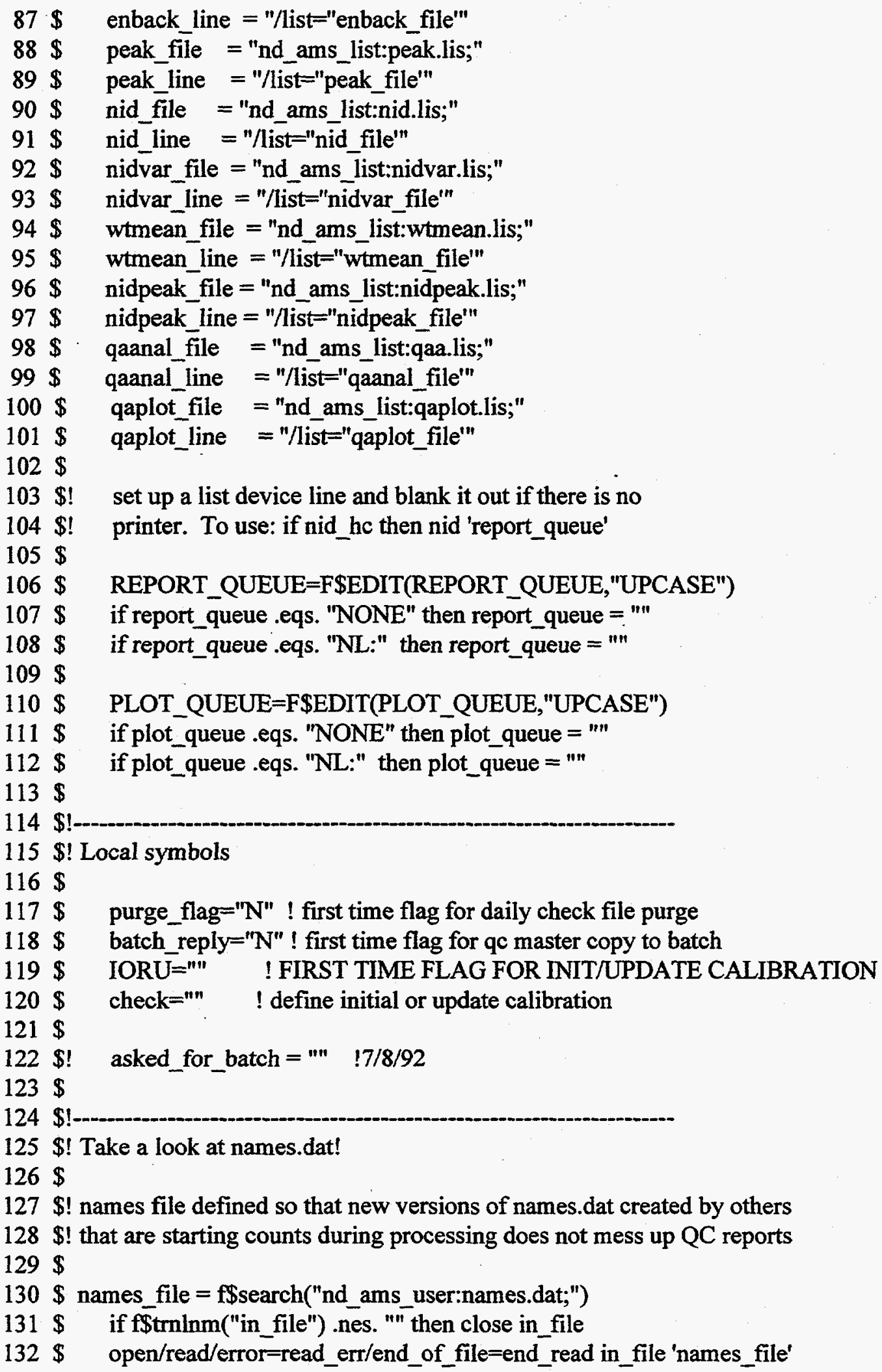


$2-82$

Appendix 2.B Listing of DCL file NDAL PROCESS.COM, file as of January 10, 1996

$133 \$$

$134 \$$

135

mca/command "erase ALPHA0"-

"delete/region ALPHAO" "set label ""

111

$136 \$$

$137 \$$ IF ON_ESP_DISPLAY THEN

@ND_AMS_COM:NDAL_CLEAR_REGION_ENERGY.COM

$138 \$$

$139 \$$ if dis_ok then display alpha0

$140 \$$

$141 \$ !$

$142 \$ ! 7 / 8 / 92$

143 \$! The configuration name has the following format:

144 \$! type_BATCH-ID\$SAMPLE-ID_library, e.g., S_BATCH-ID\$SAMPLE-ID_uu

$145 \$$ ! Types are now possibly more than one character.

$146 \$$

$147 \$ !$ jxk 8/22/95 modify messages from background processing so

$148 \$$ ! that a single file is generated and printed at the end

$149 \$$ ! create file for notice messages from background history processing

$150 \$$ if $\mathbf{f}$ edit(\$Sextract( $0,1, \mathrm{y} 12$ bioassay), "upcase") .eqs. "Y" -

151 .or. $\mathrm{f}$ Sedit(f $\$$ extract(0,1,new_reagent_calc),"upcase") .eqs. "Y"

$152 \$$ then

$153 \$$ jxk_temp_path = f\$logical("nd_ams_histry")

$154 \$$ ! construct file name

$155 \$$ temp_notemsg = "jxktempnotemsg.dat"

$156 \$$ jxk_temp_notemsg = "'jixk_temp_path'"+"'temp_notemsg'"

$157 \$$ ! show sym jxk_temp_notemsg

$158 \$$ ! first, delete all old copies

$159 \$$ jxkcolast $=$ ";*"

$160 \$$ jxkfdel = "'jxk_temp_notemsg'"+"'"jxkcolast"

$161 \$ !$ show sym jxkfdel

$162 \$ ! \quad$ if (f\$search(jxkfdel) .nes. "') then show sym jxkfdel

$163 \$$ if (\$search(jxkfdel) .nes. "') then delete 'jxkfdel'

$164 \$$

$165 \$ !$ then, open new file

$166 \$$ ! first, have to open it with fortran. Otherwise, file

$167 \$$ ! attributes are wrong

$168 \$$ file_fortopen = "'jxk_temp_notemsg"

$169 \$$ ! show sym file_fortopen

170 \$ RUN ND_AMS_EXE:fortopen

$171 \$$ ! $\quad \mathrm{r}$ fortopen.exe

$172 \$$

$173 \$$ !

open/write jxknotefile 'jxk_temp_notemsg'

$174 \$$ ! then open it with append to preserve attributes

$175 \$$ open/append jxknotefile 'jxk_temp_notemsg'

$176 \$$

$177 \$$ ! write header lines to file 
Appendix 2.B Listing of DCL file NDAL PROCESS.COM, file as of January 10, 1996

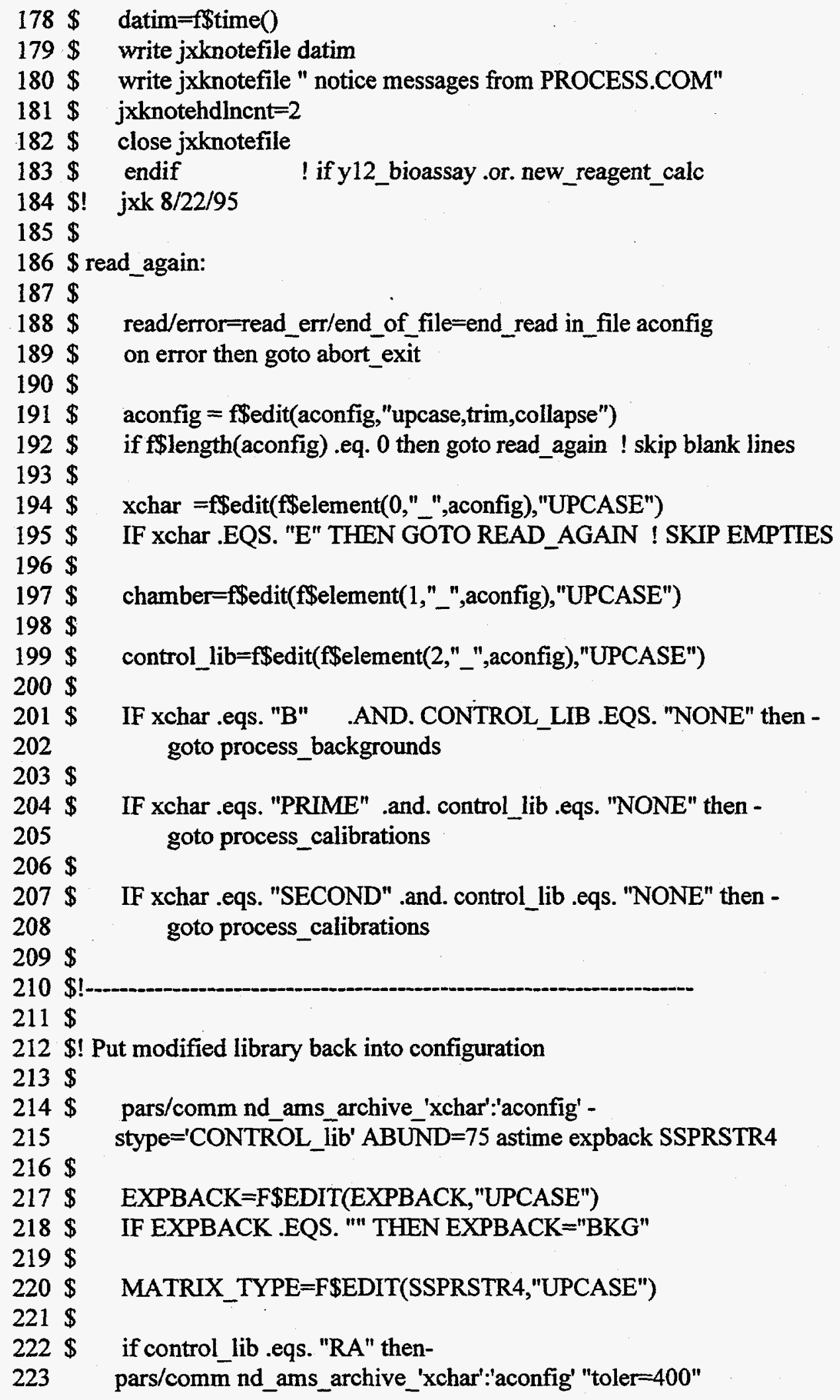

$212 \$$ ! Put modified library back into configuration

$213 \$$

$214 \$$

215

$216 \$$

$217 \$$

$218 \$$

$219 \$$

$220 \$$

$221 \$$

$222 \$$

pars/comm nd_ams_archive_xchar':'aconfig' stype $=$ 'CONTROL_

\section{EXPBACK=F\$EDIT(EXPBACK,"UPCASE")}

IF EXPBACK .EQS. "" THEN EXPBACK="BKG"

$$
\text { MATRIX_TYPE=FSEDIT(SSPRSTR4,"UPCASE") }
$$

if control_lib .eqs. "RA" thenpars/comm nd_ams_archive_'xchar':'aconfig' "toler $=400 "$ 
Appendix 2.B Listing of DCL file NDAL_PROCESS.COM. file as of January 10, 1996

$224 \$$

$225 \$$

226

227

$228 \$$

$229 \$$

$230 \$$

$231 \$$

$232 \$$

$233 \$$

$234 \$$

$235 \$$

@ND_AM

$236 \$$

$237 \$$

$238 \$$

$239 \$$

$240 \$ !$

$241 \$$

$242 \$$

$243 \$$

$244 \$$

$245 \$$

$246 \$$

$247 \$$

$248 \$ !$

$249 \$$

250 \$ read_err:

$251 \$$

$252 \$$

$253 \$$

$254 \$$

$255 \$$

$256 \$$

$257 \$ !$

$258 \$$ !

$259 \$$

$260 \$ !$

$261 \$$

262 \$ end_read:

$263 \$$

$264 \$$

$265 \$$

$266 \$$

$267 \$$

$268 \$$

then

endif

say "'"

exit
MCA/COMMAND "MOVEnd_ams_archive_"xchar':"aconfig' ALPHA0""DELETE/REGION ALPHA0" -

"set label "'Spectrum "aconfig"'"

if fermco .and. control_lib .eqs. "RA"

set_user

pars/scr/fpl=nd_ams_fpl:ndal_alpha_sample.fpl alpha0

IF ON_ESP_DISPLAY THEN

say" You are looking at alpha spectrum "+bold_on+"aconfig'+bold_off+ "

IF xchar .eqs. " $D$ " then goto process_dailys

IF xchar .eqs. "B" then goto process_backgrounds

IF xchar .eqs. "PRIME" then goto process_calibrations

IF xchar .eqs. "SECOND" then goto process_calibrations

goto process_samples

say " $\quad$ Error reading (nd_ams_user:NAMES.DAT)"

if f $\$$ trnlnm("in_file") .nes. "' then close in_file

if $\mathbf{\$}$ trnlnm("qa_file") .nes. "" then close qa_file

jxk 9/7/95 exits changed to goto master_exit

goto master_exit

jxk 9/7/95

say " The file (nd_ams_user:NAMES.DAT) is empty"

if $f$ trnlnm("in_file") .nes. "" then close in_file

if $\mathbf{f}$ trnlnm("qa_file") nes. "" then close qa_file 
269 \$ ask_do_qa:

$270 \$$

$271 \$$ qc plots = "Y"

$272 \$$ if xchar .eqs. "B" then goto do_background_qa

$273 \$$ if xchar .eqs. " $D$ " then goto do_daily_qa

$274 \$$

275 \$ask_qc_plots:

$276 \$$

$277 \$$ ask qc_plots -

278 " Generate QC Plots \& Reports $[(\mathrm{Y}) / \mathrm{N}]$ \# "

$279 \$$ qc _plots = F\$EDIT(F\$EXTRACT $\left(0,1, q c \_\right.$plots),"UPCASE")

$280 \$ \quad$ IF qc_plots .eqs. "" then qc_plots="Y"

$281 \$$ IF qc_plots.EQS. "Y" THEN GOTO DO_QA

282 \$ IF qc plots.EQS. "N" THEN GOTO DO_QA

$283 \$$ SAY "'"

284 \$ SAY " Invalid response, try again..."

$285 \$$ goto ask_qc_plots

$286 \$$

287 \$ DO_QA:

$288 \$$

$289 \$$ if xchar .eqs. "PRIME" then goto do_weekly_qa

$290 \$$ if $x$ char .eqs. "SECOND" then goto do_weekly_qa

291 \$ goto do_sample_qa

$292 \$$

$293 \$$ !

$294 \$$

295 \$PROCESS_DAILYS:

$296 \$$

$297 \$$ PARS/COMMAND ALPHA0 "DISPECOUNT=16384" "DISPMODE=LIN"

$298 \$$

$299 \$$ if (.not. purge_flag .and. .NOT. DEMO)

300 \$ then

301 \$ SAY -

302 " one moment ....."+bold_on+"purging files"+bold_off+"....."

$303 \$$ PURGE/keep=2/EXCLUDE=(MCA*.LOG,NET*.LOG) -

304 nd_ams_user:*.cnf,nd_ams_user:names.dat

$305 \$$ PURGE/before=yesterday nd_ams_list:*.lis

$306 \$$ delete/before=yesterday nd_ams_archive_alpha:alpha*_d_*.cnf;"*/log

307 \$ purge_flag="Y"

308 \$ endif ! purge_flag

$309 \$$

$310 \$$

$311 \$$

$312 \$$

$313 \$$

314 \$ qaxfer ALPHA0 nd_ams_qa_d:D'chamber' 
$315 \$$

$316 \$$ ! Save current acq start time and ajust for qaxfer

$317 \$$

$318 \$$

$319 \$$

$320 \$$

$321 \$$

$322 \$$

$323 \$$

$324 \$$

$325 \$$

$326 \$$

$327 \$$

$328 \$ !$

$329 \$$

$330 \$$

$331 \$$

$332 \$$

$333 \$$

$334 \$ !$

$335 \$$

336 \$PROCESS_BACKGROUNDS:

$337 \$$

$338 \$$ ! fetch the most recent file for selected detector number if date=NONE

$339 \$$

$340 \$$ if control_lib .eqs. "NONE"

$341 \$$ then

342 \$@nd_ams_com:ndal_getlast "'xchar';"chamber"" "cnf" "nd_ams_archive_"xchar""

343 \$@nd_ams_com:ndal_extract_.'ndlastfile'

344 \$@nd_ams_com:ndal_extract_. 'extraction'

$345 \$$ aconfig="'"xchar'_"chamber'_"extraction"'-".cnf"

$346 \$$ say "'"

347 \$ say"

348 \$ endif

$349 \$$

$350 \$$ MCA/COMMAND "MOVE nd_ams_archive_"xchar':"aconfig' ALPHA0"-

351

352

$353 \$$

$354 \$$

355

$356 \$$

$357 \$ !$

$358 \$$

$359 \$ !$

$360 \$$
You are looking at alpha spectrum "+bold_on+"aconfig'+bold_off+ " "DELETE/REGION ALPHA0" -

"set label "'Spectrum "aconfig"""

PARS/COMMAND ALPHA0 "DISPECOUNT=32" "DISPMODE=LIN" -

"DETECTOR="CHAMBER"" "DETNAME="CHAMBER"" 


\section{Appendix 2.B Listing of DCL file NDAL PROCESS.COM, file as of January 10, 1996}

$361 \$$ if plot_hc .and. plot_queue .nes. "'t

$362 \$$ then

$363 \$$ if dvm

$364 \$$ then

$365 \$$

$366 \$$

$367 \$$

$368 \$$

$369 \$$

$370 \$$

$371 \$$

$372 \$$

373

$374 \$$

$375 \$$

$376 \$$

377 \$skip_BKG_plot:

378 \$

$379 \$$

$380 \$$

$381 \$ !$

$382 \$$

$383 \$$

$384 \$$

$385 \$$

$386 \$$

$387 \$$

388

say "'"

say " System is now developing a plot on "+bold_on+"Alpha0"+bold_off+" plot/alpha/left $=3500 /$ right $=6500 /$ nonid/noregion ALPHAO 'plot_file'

else

goto skip_BKG_plot

display nd_ams_archive_'xchar':'aconfig' -

/linear/proto $=$ 'plot_proto"plot_line'/land/start $=3$

endif !dvm

endif ! he flag

$389 \$$

390

$391 \$$

$392 \$$

$393 \$$

$394 \$$

$395 \$ !$

generate channel by channel listings dvm $5 / 93$

if list_hc .and. report_queue .nes. ""

then

if dvm

then

list nd_ams_archive_'xchar':'aconfig' -

/head=nd_ams_headers:chan 1 .hdr/col $=10 /$ end $=600$ 'chan1_line'

list nd_ams_archive_'xchar':'aconfig' -

hhead=nd_ams_headers:chan $2 . h d r / c o l=10 /$ start $=601$ 'chan2' line'

else

list nd_ams_archive_'xchar':'aconfig' 'list_line'

endif !dvm

endif !hc

$396 \$$

$397 \$$

$398 \$$

$399 \$ !$

$400 \$$

$401 \$ !$

$402 \$ !$

$403 \$$

$404 \$$

$405 \$$

$406 \$$

Let's do background checks on actinide regions of interest,

Build a background file

copy nd_ams_headers:reports.hdr nd_ams_list:background.lis

copy nd_ams_headers:reports.hdr 'background_lis'

pars $/$ com alpha0 stype $=$ "Backgrounds"

@nd_ams_regions:BACKGROUND.REGION 
$407 \$$ ! peak/region/midpoint/type=gross/header=nd_ams_headers:bkgnds.hdr alpha0 'bkgnd_line'

$408 \$$ peak/region/midpoint/type=gross alpha0

$409 \$$

$410 \$ ! \quad$ append 'bkgnd_file' nd_ams_list:background.lis

$411 \$ ! \quad$ append 'bkgnd_file' 'background_lis'

$412 \$$

$413 \$$

$414 \$$

$415 \$$

$416 \$$

$417 \$$ !

$418 \$$

$419 \$$

$420 \$$

$421 \$$

$422 \$$

$423 \$$

$424 \$$

$425 \$$

$426 \$$

$427 \$$

$428 \$$

$429 \$$ !

$430 \$$

$431 \$$

$432 \$$

$433 \$ !$

$434 \$$ !

$435 \$$

delete 'bkgnd_file'

QAXFER ALPHA0 nd_ams_qa_b:B'CHAMBER'

Save current acq start time and ajust for qaxfer

pars/comm alpha 0 astime/symbol=astime_save

det=f\$integer(chamber)

$\mathrm{adc}=($ det- 1$) /$ number_of_groups +1

offset $=\operatorname{det}-($ adc- 1$) *$ number_of_groups

qaxfer_astime="1-JAN-94 00:"adc':"offset""

pars/comm alpha0 astime="'"qaxfer_astime"

QAXFER ALPHA0 nd_ams_qa_'xchar':batch_'xchar' $\quad$ 17/2/92

$440 \$$

$441 \$$

$442 \$ !$

$443 \$$ !

$444 \$$

$445 \$$

$446 \$$ !

$447 \$$

$448 \$$

$449 \$ !$

$450 \$$ !

Put back original acq start time

pars/comm alpha 0 astime="'"astime_save"'

jxk 8/9/95 create temporary file for background messages

from this sample

if f\$edit(\$\$extract $(0,1, \mathrm{y} 12$ bioassay),"upcase") .eqs. "Y" -

.or. $\$$ Sedit(f $\$$ extract( 0,1, new_reagent_calc),"upcase") .eqs. "Y"

then

jxk_temp_path $=$ \$ $\$$ logical("nd_ams_histry")

construct file name

temp_bkmsg $=$ "jxktempbkmsg.dat"

jxk_temp_bkmsg = "'jxk_temp_path'"+"'"temp_bkmsg"'

show sym jxk_temp_bkmsg

first, delete all old copies

jxkcolast $=$ ";*"

jxkfdel = "'jxk_temp_bkmsg'"+"'"jxkcolast"'

show sym jxkfdel

delete 'jxkfdel'

$451 \$ !$

then, open new file

first, have to open it with fortran. Otherwise, file

$452 \$$ attributes are wrong

file_fortopen $=$ "'jxk_temp_bkmsg'" 


\section{Appendix 2.B Listing of DCL file NDAL PROCESS.COM, file as of January 10.1996}

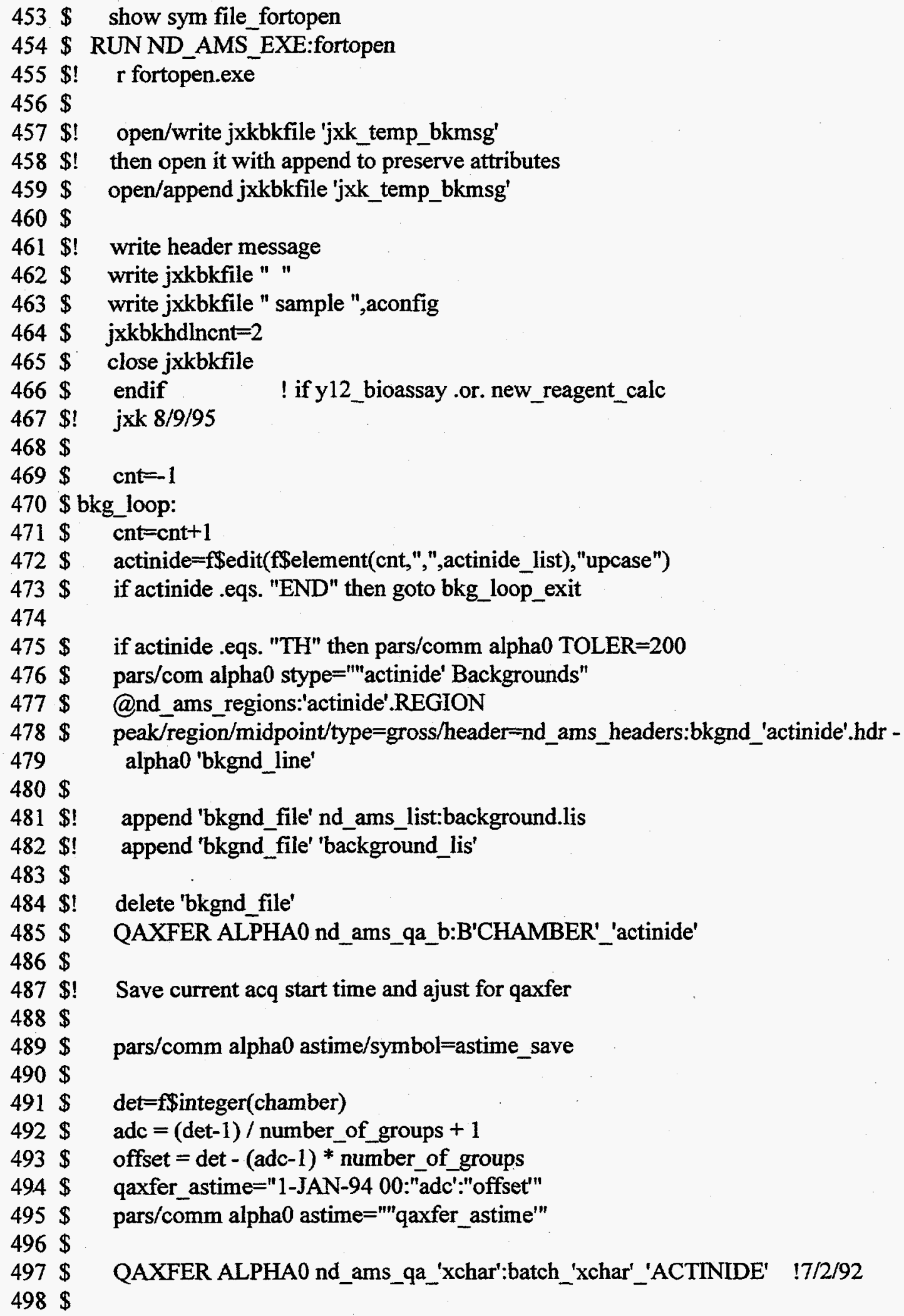


$499 \$$ ! Put back original acq start time

$500 \$$

$501 \$$ pars/comm alpha0 astime="'"astime_save""

$502 \$$

$503 \$$ ! execute jxk's process backgrounds for history .com file 6/95

$504 \$$

505 \$@nd_ams_com:ndal_bkgd_histry.com

$506 \$$

$507 \$$ ! execute jxk's process backgrounds for history .com file 6/95

$508 \$$

$509 \$$

$510 \$$

goto bkg_loop

511 \$bkg_loop_exit:

$512 \$$

$513 \$ ! \quad$ jxk $8 / 9,22 / 95$ append temporary background message file for this

$514 \$$ ! sample to message file for all samples and delete

$515 \$$ if $\mathbf{f}$ edit(\$extract $(0,1, y 12$ bioassay), "upcase") .eqs. "Y" -

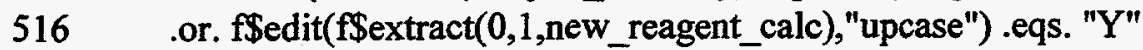

$517 \$$ then

$518 \$$ ! print/delete 'jxk_temp_bkmsg'

$519 \$$ ! close jxkbkfile

$520 \$$ ! temporary type of messages file to see contents

521 \$ type 'jxk_temp_bkmsg'

$522 \$ !$ count lines in the message file to see if it has any messages

$523 \$$ jxklinecnt $=0$

$524 \$$ open/read jxkinfile 'jxk_temp_bkmsg'

$525 \$$ ! 3 reads to make sure I count past header lines

$526 \$$ read/end_of_file=jxkbkeof jxkinfile jxkline

$527 \$$ jxklinecnt $=$ jxklinecnt +1

$528 \$$ read/end_of_file=jxkbkeof jxkinfile jxkline

$529 \$$ jxklinecnt $=$ jxklinecnt +1

$530 \$$ read/end_of_file=jxkbkeof jxkinfile jxkline

$531 \$$ jxklinecnt $=\bar{j}$ xklinecnt +1

$532 \$$

$533 \$$

$534 \$$

jxkbkeof:

$535 \$ !$ if there is more than the header line(s), append the background

$536 \$$ ! messages file for this sample to the total messages file

$537 \$$ if jxklinecnt .gt. jxkbkhdlncnt

$538 \$$ then

$539 \$$ append 'jxk_temp_bkmsg' 'jxk_temp_notemsg'

$540 \$$ endif

$541 \$$ ! don't delete jxk_temp_bkmsg here because it will be deleted

$542 \$ !$ before it is re-created. redundant construct file name and

$543 \$ !$ delete wastes time

$544 \$$ endif ! if y12_bioassay .or. new_reagent_calc 
Appendix 2.B Listing of DCL file NDAL PROCESS.COM. file as of January 10.1996

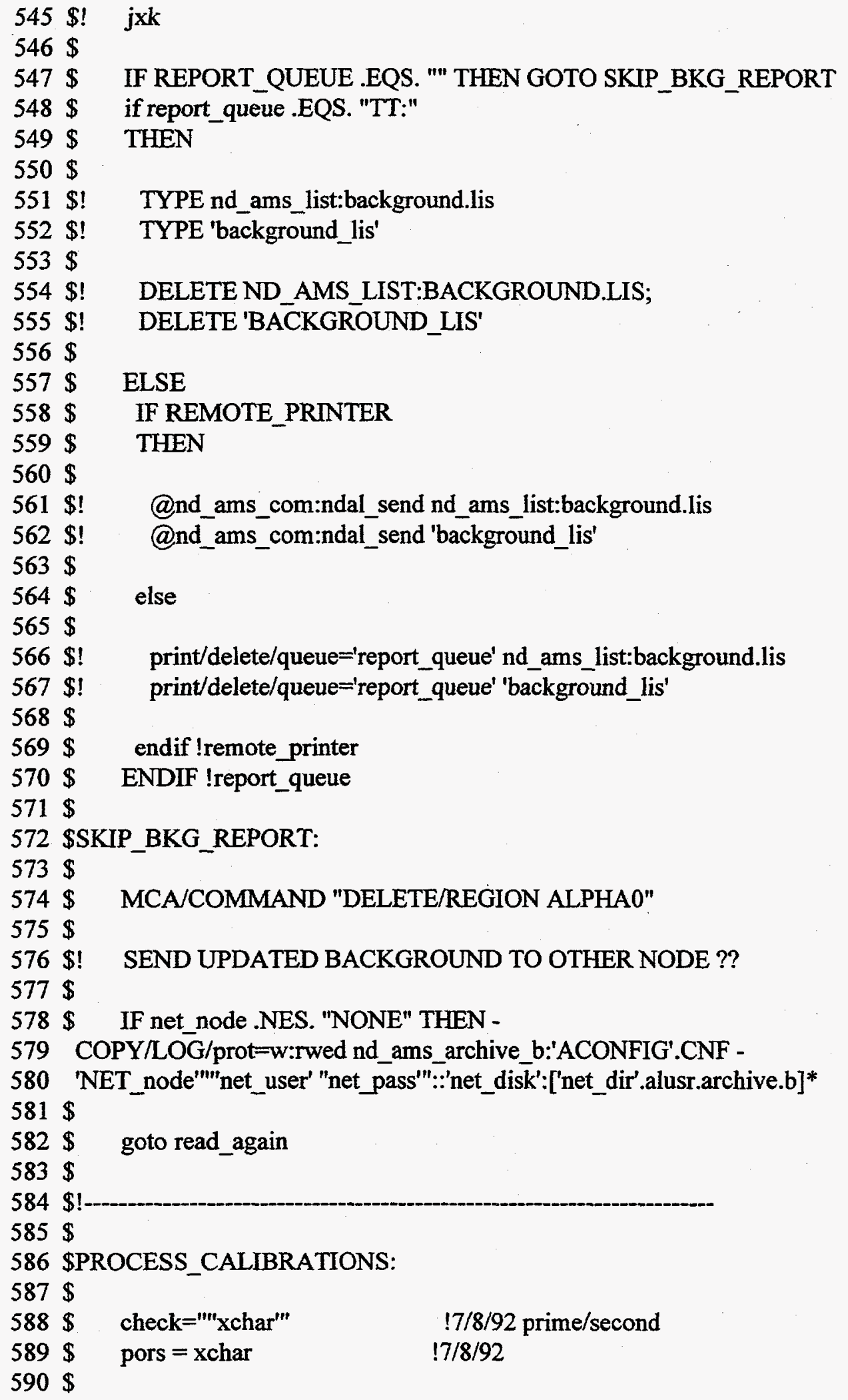


Appendix 2.B Listing of DCL file NDAL PROCESS.COM, file as of January 10, 1996

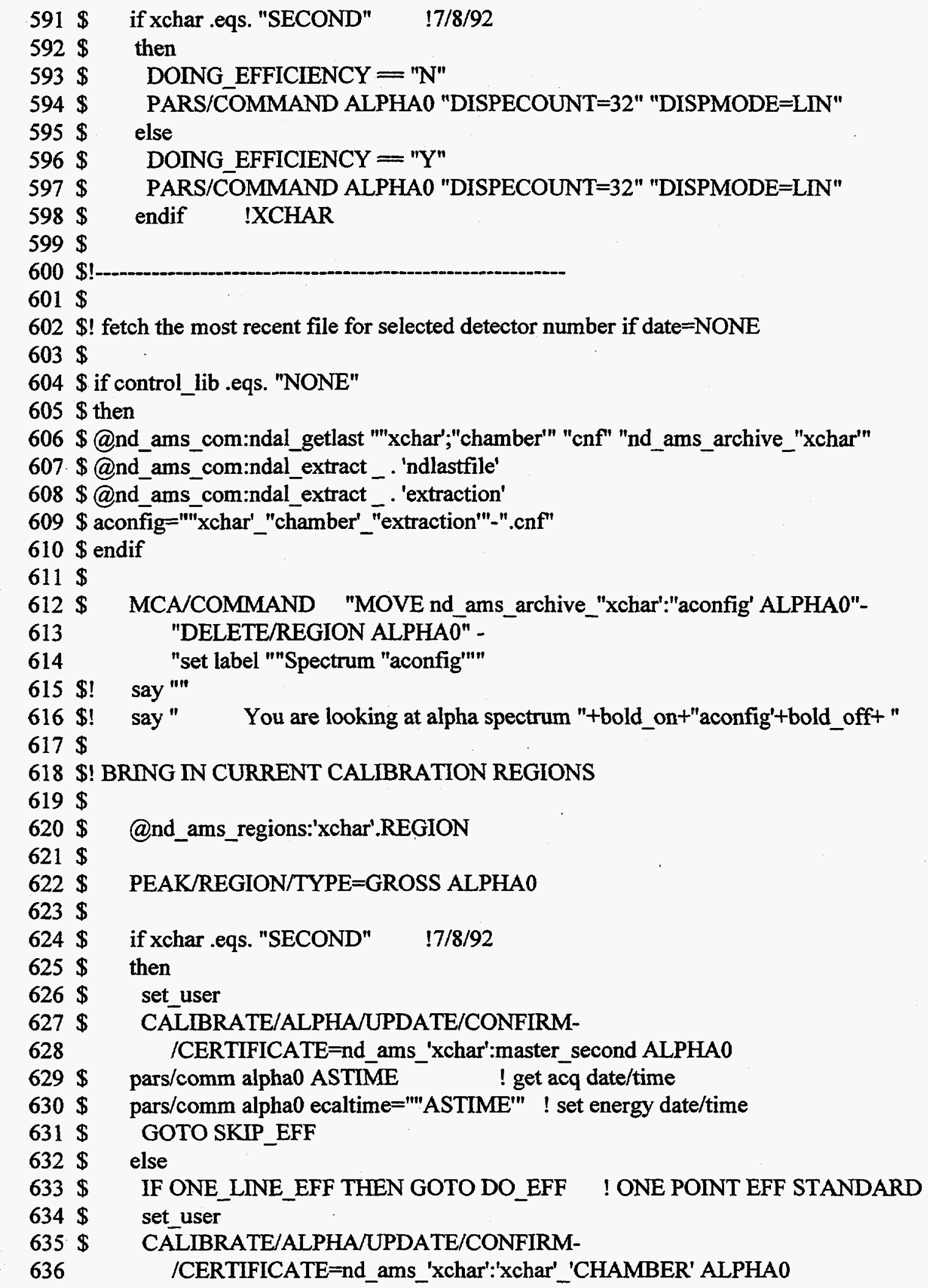


Appendix 2.B Listing of DCL file NDAL PROCESS.COM, file as of January 10, 1996

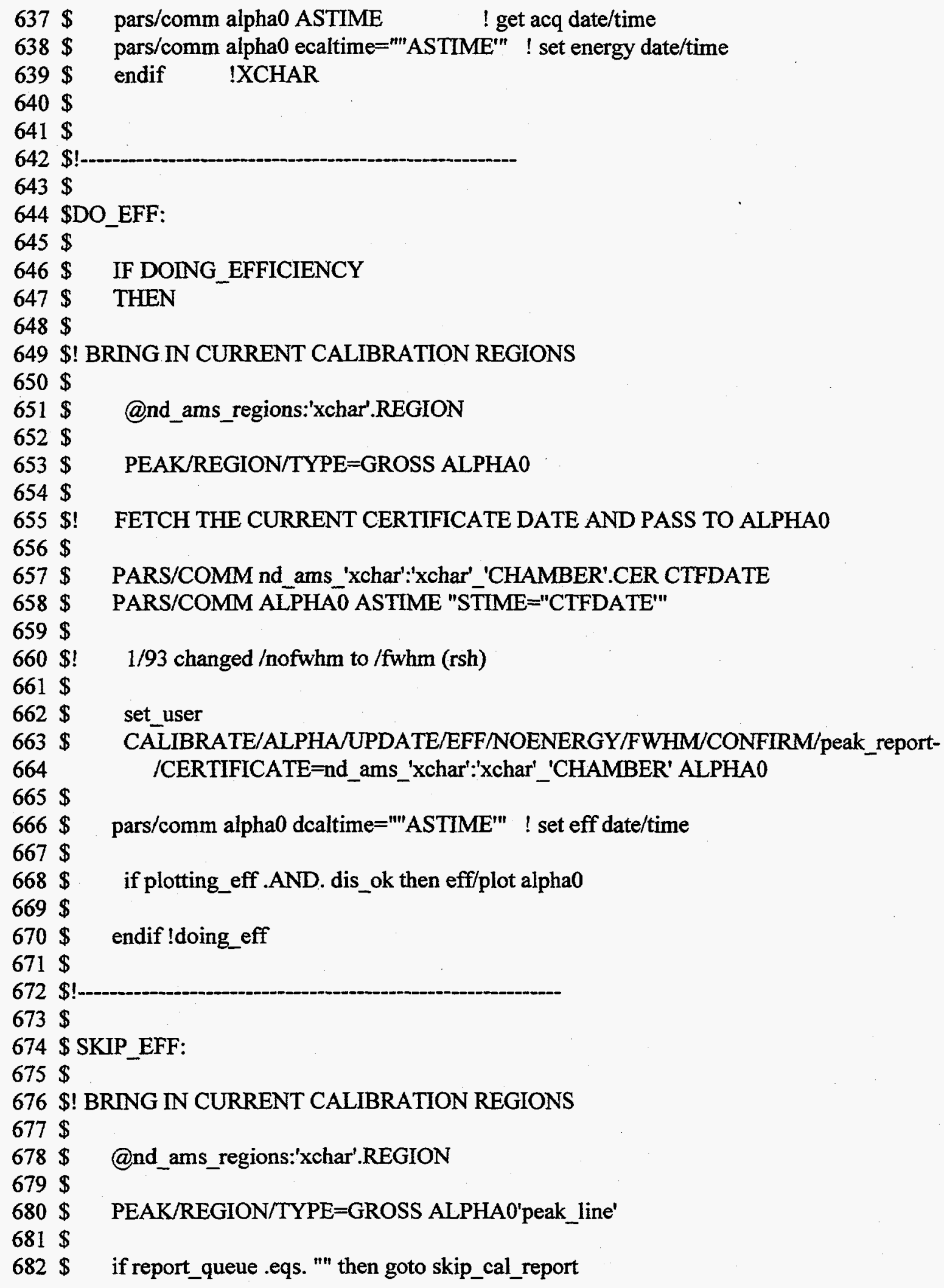




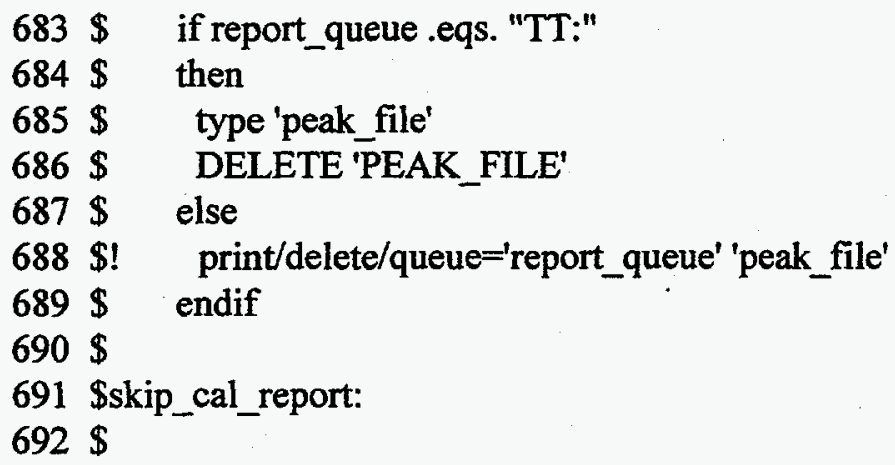

$693 \$$ ! 1/93 improved the move/partial call with new technology! (rsh)

$694 \$$

$695 \$$ move/partial alpha0/eff nd_ams_archive_'xchar':'ACONFIG'

$696 \$$ move/partial alpha0/eff nd_ams_user:1'CHAMBER'

$697 \$$

$698 \$$ if current_software

$699 \$$ then

$700 \$$ MOVE/partial/fpl=nd ams fpl:ndal_cal params_xchar'.fpl-

701

$702 \$$

703

$704 \$$

$705 \$$

$706 \$$

$707 \$$

$708 \$$

$709 \$$

$710 \$$

$711 \$$

$712 \$$

$713 \$$

$714 \$$

$715 \$$

$716 \$$

$717 \$$

$718 \$$

719

720 \$

721

$722 \$$

$723 \$$

$724 \$$

$725 \$$

$726 \$$

$727 \$$

728 \$!

ALPHA0 nd_ams_archive_'xchar':'ACONFIG'

MOVE/partial/fpl=nd_ams_fpl:ndal_cal_params_xchar'.fplelse

move ALPHAO nd_ams_archive 'xchar':'ACONFIG'

move ALPHA0 nd_ams_user:1'CHAMBER'

endif

$\operatorname{det}=\mathbf{F}$ Sinteger(chamber)

adc $=($ det- 1$) /$ number_of_groups +1

offset $=$ det $-($ adc- 1$) *$ number_of_groups

START $=$ 'group_size' * (offset -1$)+1$

if start .eq. 1

then

if current_software

then

MOVE/partial/fpl=nd_ams_fpl:ndal_cal_params_xchar'.fplalpha0 nd_ams_setup:setup_alpha'adc'

MOVE/partial/fpl=nd_ams_fpl:ndal_cal_params_xchar'.fplalpha0 alpha'adc'

else

move/part alpha0/calib nd_ams_setup:setup_alpha'adc'

move/part alpha0/calib alpha'adc'

endif

endif

SEND UPDATED HEADER TO OTHER NODE ?? 
Appendix 2.B Listing of DCL file NDAL PROCESS.COM, file as of January 10, 1996

$729 \$$

730 \$ IF NET_node .NES. "NONE"

731 \$ THEN

732 \$ COPY/LOG/prot=w:rwed nd_ams_user:1'CHAMBER'.CNF -

733 NET_node'"'net_user' "net_pass"'::'net_disk':['net_dir'.alusr]*

$734 \$$ COPY/LOG/prot=w:rwed nd_ams_archive_xchar':'ACONFIG'.cnf -

735 NET_node'"'"net_user' "net_pass'"::'net_disk':['net_dir'.alusr.archive.'xchar']*

736 \$ endif

$737 \$$

$738 \$$ NID/LIB=nd_ams_lib:'xchar'.NLB/nolist ALPHA0

$739 \$$

$740 \$ !$

$741 \$$

742 \$!generate calibration spectral plot dvm 5/93

$743 \$$

$744 \$$ if plot_hc .and. plot_queue .nes. ""

$745 \$$ then

$746 \$$ if $\mathrm{dvm}$

$747 \$$ then

$748 \$$ say "'

$749 \$$ say" System is now developing a plot on "+bold_on+"Alpha0"+bold_off + "

$750 \$$

$751 \$$

752 \$ GOTO SKIP_CALIBRATE_PLOT

$753 \$$ display nd_ams_archive_'xchar':'aconfig' -

754

$755 \$$

$756 \$$

$757 \$$

758 \$SKIP_CALIBRATE_PLOT:

$759 \$$

$760 \$$

$761 \$$

$762 \$$

$763 \$$

$764 \$ !$

$765 \$$

$766 \$$

$767 \$$

$768 \$$

$769 \$$

$770 \$$

$771 \$$

$772 \$$

$773 \$$

$774 \$$

QAXFER ALPHA0 nd_ams_qa_'xchar':'xchar"CHAMBER'

Save current acq start time and ajust for qaxfer

pars/comm alpha0 astime/symbol=astime_save

det $=\mathbf{f}$ integer(chamber)

$\operatorname{adc}=($ det -1$) /$ number_of_groups +1

offset $=$ det $-(\operatorname{adc}-1) *$ number_of_groups

qaxfer_astime $=" 1-J A N-9400: "$ adc':"offset"

pars/comm alpha0 astime="'"qaxfer_astime'"'

QAXFER ALPHA0 nd_ams_qa_'xchar':batch_'xchar' !7/2/92 
Appendix 2.B Listing of DCL file NDAL PROCESS.COM, file as of January 10.1996

$775 \$$

$776 \$$

$777 \$$

$778 \$$ pars/comm alpha0 astime="'"astime_save"'

$779 \$$

$780 \$$

$781 \$$

$782 \$ !$

$783 \$$

$784 \$ !$

$785 \$$

786 \$PROCESS_SAMPLES:

$787 \$$

$788 \$$

$789 \$$

$790 \$$

$791 \$$

$792 \$$

$793 \$$

794

795

796

$797 \$$

$798 \$ !$

$799 \$ !$

$800 \$$ !

$801 \$ !$

$802 \$$

$803 \$$

$804 \$$

805 \$ matrix_loop:

$806 \$$

$807 \$$ cnt $=$ cnt +1

$808 \$$ valid matrix=f\$edit(f\$element(cnt,",",matrix list),"upcase")

$809 \$$ if valid matrix .eqs. "END" then goto invalid_matrix_type

$810 \$$ if valid_matrix .eqs. matrix_type then goto matrix_type_ok

$811 \$$ goto matrix_loop

$812 \$$

813 \$invalid_matrix_type:

$814 \$$

$815 \$$

$816 \$$

$817 \$$

$818 \$$

$819 \$$

$820 \$$
IF MATRIX_TYPE .EQS. "" THEN MATRIX_TYPE="NONE"

say "'

say " Unsupported matrix type ("+bold_on+"'matrix_type"'+bold_off+")"

say " Valid matrices are as follows:"

say bold_on+" "matrix_list'"+bold_off

ask reply" Hit return to make correction \# " 


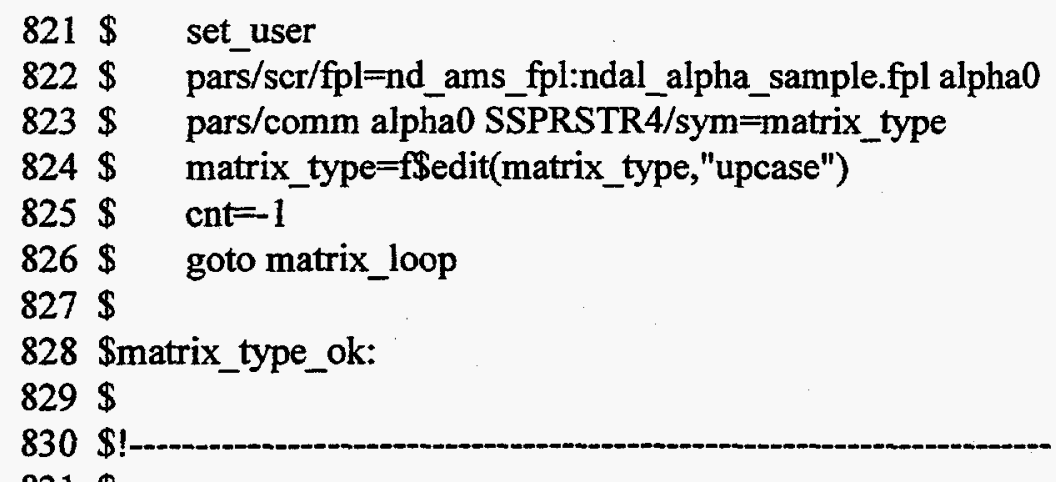

$831 \$$

$832 \$ !$ CREATE SUPPORTING (YIELD) QC FILES

$833 \$$

834 \$ IF f \$search -

835 ("nd_ams_qa_y:BATCH_y_"CONTROL_LIB'_"matrix_type'.qaf") .eqs. "" 836 \$ THEN

$837 \$$ copy/LOG -

838 nd_ams_qa y:master_y_'control_lib' 'matrix_type'.qaf -

839 nd_ams_qa y:batch_y_'control_lib'_'matrix_type'.qaf

840 \$ endif

$841 \$$

842 \$ IF f $\$$ search -

843 ("nd_ams_qa_y:"batch_id_num'_y_"CONTROL_LIB'_matrix_type'.qaf;*") .eqs. ""

$844 \$$ THEN

$845 \$$ copy/LOG -

846 nd_ams_qa_y:master_y_control_lib'_matrix_type'.qaf -

847 nd_ams_qa_y:'batch_id_num'_y_control_lib'_matrix_type'.qaf

848 \$ endif

$849 \$$

$850 \$ !$

$851 \$$

$852 \$ !$ CREATE SUPPORTING (CONTROL) QC FILES

$853 \$$

854 \$ IF f\$search ("nd_ams_qa_C:BATCH_C_"CONTROL_LIB'_"matrix_type'.qaf") .eqs. "'" 855 \$ THEN

$856 \$$ copy/LOG -

857 nd_ams_qa_C:master_C_'control_lib'_matrix_type'.qaf -

858 nd_ams_qa_C:batch_C_control_lib'_matrix_type'.qaf

859 \$ endif

$860 \$$

$861 \$ !$

$862 \$$

$863 \$ !$

CREATE SUPPORTING (REAGENT) QC FILES

$864 \$$

865 \$ IF f $\$$ search ("nd_ams_qa_R:BATCH_R_"CONTROL_LIB'_"matrix_type'.qaf") .eqs. "' 866 \$ THEN 


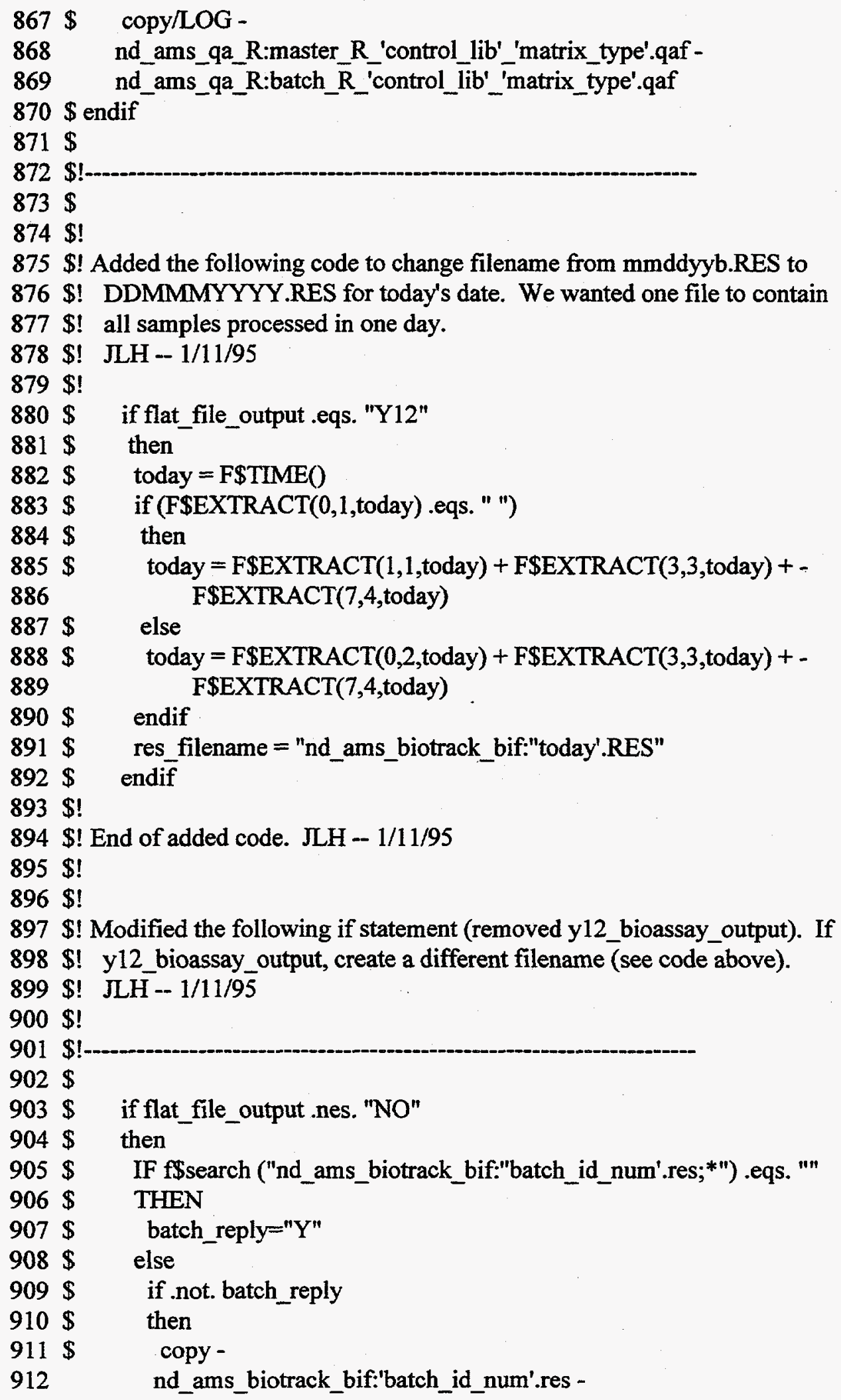




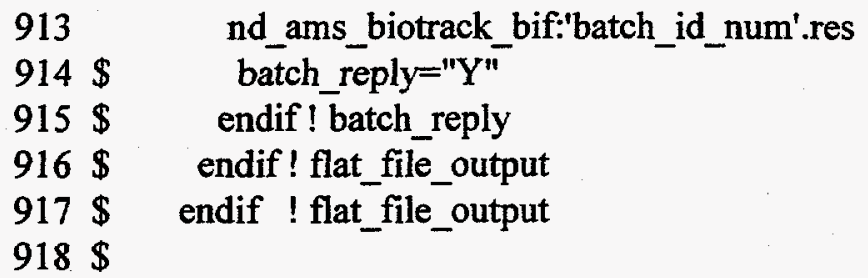

$919 \$ !$

$920 \$$

$921 \$$ \$ SRS treats AM/NP as GROSS spiked/unspiked

$922 \$$

923 \$ if flat_file_output .eqs. "SRS".AND. -

924 (control_lib .eqs. "GROSS-AM" .OR. CONTROL_LIB .EQS. "GROSS-NP")

$925 \$$ then

926 \$ IF f\$search ("nd_ams_qa_y:"batch_id_num'_y_"CONTROL_LIB'_control.qaf;*") .eqs. ""

$927 \$$ THEN

$928 \$$ copy/LOG -

929 nd_ams_qa_y:master_y_'control_lib' 'matrix_type'.qaf -

930 nd_ams_qa_y:'batch_id_num'_y_control_lib'_control.qaf

$931 \$$ endif ! srs_bioassay_output

932 \$ endif! srs_bioassay_output

$933 \$$

$934 \$$

$935 \$$

$936 \$$ ! setup regions for plots moved here to allow more time to examine

$937 \$$ ! regions without breaking up program flow dvm 5/93 it corporation

$938 \$$

$939 \$$

$940 \$$

@nd_ams_regions:'CONTROL_LIB'.REGION

$941 \$ !$

$942 \$$ ! dump list of whole spectrum ?? Set list_hc via maintenance menu.

$943 \$ !$ generate channel by channel listings

$944 \$$ ! printout moved to below for setting printout order

$945 \$$

$946 \$$

$947 \$$

$948 \$$

949

$950 \$$

951

$952 \$$

$953 \$$

$954 \$$

if $\mathrm{dvm}$

then

if list_hc then list nd_ams_archive_xchar':'aconfig' /head=nd_ams_headers: chan $1 . h d r / c o l=10 /$ end $=600$ 'chan1_line'

$955 \$$

$956 \$ !$

$957 \$$

958 \$! start building a peaks search report if necessary dvm 5/93 


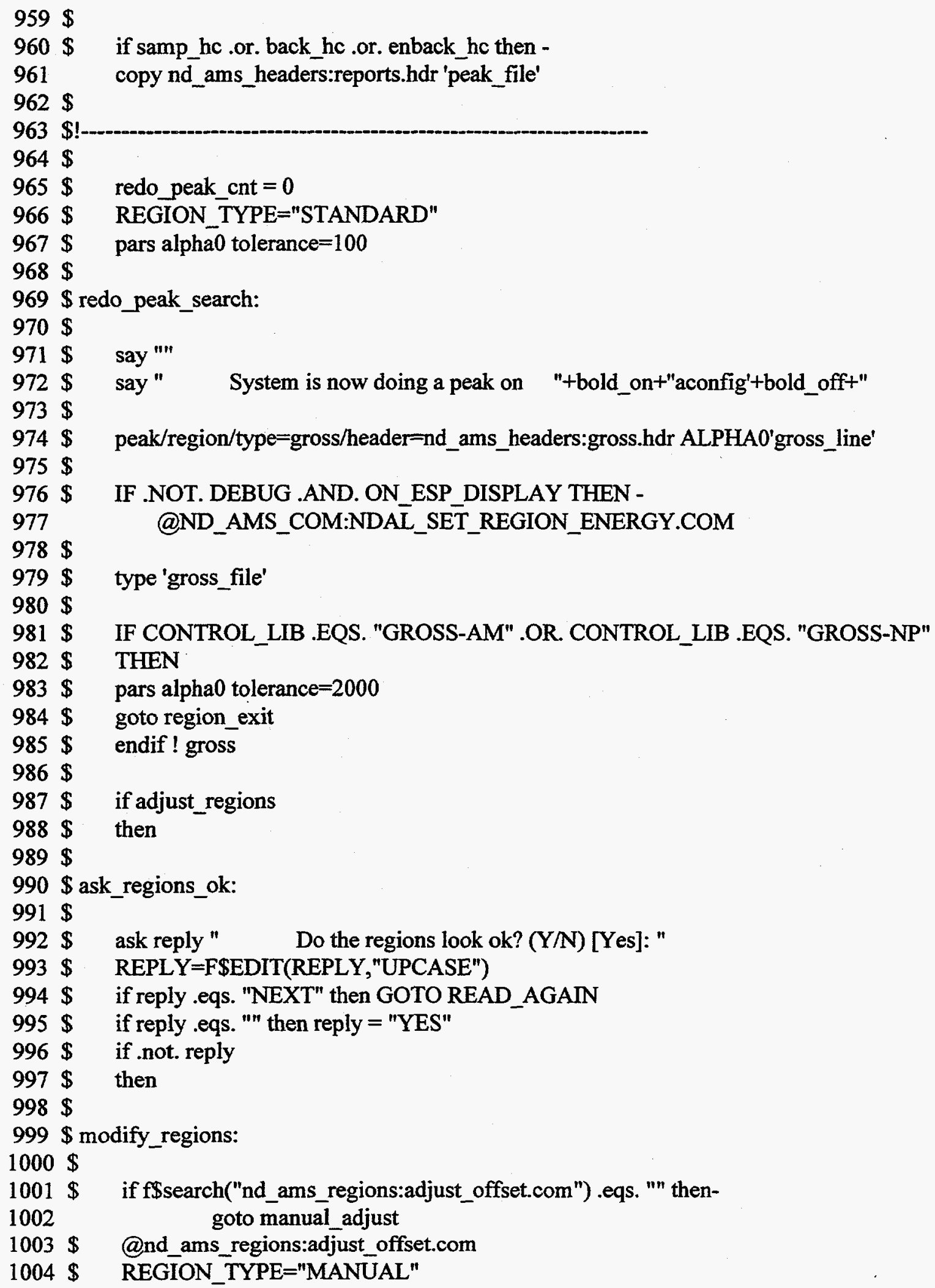


Appendix 2.B Listing of DCL file NDAL_PROCESS.COM, file as of January 10, 1996

$1005 \$$ goto redo_peak_search

$1006 \$$

1007 \$ manual_adjust:

$1008 \$$

$1009 \$$ redo_peak_cnt $=$ redo_peak_cnt +1

$1010 \$$ if redo_peak_cnt .eq. 1

$1011 \$$ then

1012 \$ REGION_TYPE="EXPANDED"

$1013 \$$ pars alphä0 tolerance $=125$

$1014 \$ @ N D \_A M S$ \&EGIONS:'control_lib'_WIDE.REGION

$1015 \$$ goto redo_peak_search

$1016 \$$ else

$1017 \$$ REGION_TYPE="MANUAL"

$1018 \$$ pars alphā0 tolerance $=150$

$1019 \$$ say "'"

$1020 \$$ say " To manually change the regions for this sample. Use the mouse to"

$1021 \$$ say " adjusted the preset regions to their new position. When finished"

$1022 \$$ ask $x$ " hit return to continue..."

$1023 \$$ goto redo_peak_search

$1024 \$$ endif ! redo_peak_cnt

$1025 \$$

$1026 \$$

$1027 \$$

$1028 \$$

$1029 \$ !$

$1030 \$$ ! Now... Append/get rid of peak report dvm 5/93

$1031 \$$ if samp_hc then append 'gross_file' 'peak_file'

$1032 \$$ delete 'gross_file'

$1033 \$ !$

$1034 \$$

1035 region_exit:

$1036 \$$

1037 \$IF .NOT.colorado_doh THEN GOTO SKIP_colorado_doh

1038 \$ ask reply " Would you like to delete a peak region $(\overline{\mathrm{Y} /}[\mathrm{N}])$ \# "

1039 \$ reply=f\$edit(f $\$$ extract(0,1,reply),"upcase")

$1040 \$$ if .not. reply then goto skip_colorado_doh

1041 \$ask_next_region:

1042 \$ ask record " Enter region number to be deleted or return when finished \# "

1043 \$ if record .eqs. "' then goto skip_colorado_doh

$1044 \$$ pars/comm alpha0 psarea/record $=$ 'record'

1045 \$ say " Region "record' contains "psarea' counts"

1046 \$ ask reply" Is this the correct region $(\mathrm{Y} / \mathrm{N})$ \#"

$1047 \$$ if not. reply then goto ask_next_region

$1048 \$$ pars/comm alpha0 psarea $=" 0 " /$ record $=$ 'record' psctss=" $0 " /$ record='record'

1049 \$ goto ask_next_region

1050 \$skip_colorado_doh: 
Appendix 2.B Listing of DCL file NDAL_PROCESS.COM, file as of January 10, 1996

$1051 \$$

$1052 \$ \mathrm{sac}=" 1.0 "$

1053 \$ assign/nolog 'sac' sac

$1054 \$$

1055 \$IF .NOT. TMA_EBERLINE.OR. CONTROL_LIB .NES. "RA" THEN GOTO SKIP_RADIUM

$1056 \$$

1057 \$ask sac " Enter SAC factor (default=1.0) \# "

1058 if sac .eqs. "" then $\mathrm{sac}=" 1.0 "$

$1059 \$$ assign/nolog 'sac' sac

$1060 \$$ SAC $=$ f $\$$ trnlnm("SAC")

$1061 \$$

1062 \$ say "'"

1063 \$ say"

1064 \$ say "

System is now doing a RA_MANIP on "+bold_on+"aconfig"+bold_off+"

$1065 \$$

RA_MANIP ALPHA0=ALPHA0*"sac' for (AREA \& CPS)."

1066 \$ RUN ND_AMS_EXE:RA_MANIP

$1067 \$$

1068 \$ say ""

1069 \$ say " System is now reporting adjusted peaks on "+bold_on+"aconfig'+bold_off+"

$1070 \$$

$1071 \$$ ndreport/peak ALPHA0'nid_line' !rsh, added : for In

$1072 \$$

$1073 \$$ ! type 'nid_file'

$1074 \$$

$1075 \$$

$1076 \$$

$1077 \$$ ! Fetch chamber number - and batch id number dvm 5/93

$1078 \$$

1079 \$SKIP_RADIUM:

$1080 \$$

$1081 \$$ DET=F\$NTEGER(CHAMBER)

$1082 \$$ @ND_AMS_COM:NDAL_PREFIX.COM 'DET'

$1083 \$$ NUMBER=""PRFX"'DET"

$1084 \$$

$1085 \$$ ! Fetch most recent background if necessary

$1086 \$$

$1087 \$$ if $\$$ edit(expback, "upcase") .eqs. "BKG"

1088 \$ then

1089 \$@nd_ams_com:ndal_getlast "b;"number" "cnf" "nd_ams_archive_b"

$1090 \$$ backgnd = "'ndlastfile"'

$1091 \$$ pars/comm alpha0 expback="'"backgnd"'

1092 \$ else

1093 \$ backgnd = "'expback"

1094 \$ endif

$1095 \$$

1096 \$ @nd_ams_com:ndal_extract ] . 'backgnd' 
Appendix 2.B Listing of DCL file NDAL PROCESS.COM. file as of January 10, 1996

$1097 \$$ backgnd = "nd_ams_archive_b:"+extraction+".cnf"

$1098 \$$

$1099 \$$ define/nolog bkg 'backgnd'

$1100 \$$ define/nolog areas 'backgnd'

$1101 \$$

$1102 \$ !$

1103 \$! PLACE CURRENT SELECTED REGIONS INTO BACKGROUND SPECTRUM

$1104 \$$ ! modified to use bkgnd_file dvm 5/93

$1105 \$$

$1106 \$$ MOVE/PARTIAL ALPHA0/REGIONS BKG: !rsh, added : for $\ln$

$1107 \$$

$1108 \$$ peak/region/midpoint/type=gross/header-nd_ams_headers:bkgnd.hdr -

1109 bkg:'bkgnd_line' !rsh, added : for ln

1110 \$ type 'bkgnd_file'

$1111 \$$

$1112 \$ !$

$1113 \$$ ! Now... Append/get rid of background report dvm 5/93

$1114 \$$ if back_he then append 'bkgnd_file' 'peak_file'

$1115 \$$ delete 'bkgnd_file'

$1116 \$$ !

$1117 \$$

$1118 \$$ !

$1119 \$$

$1120 \$$

$1121 \$$

$1122 \$$

$1123 \$$

$1124 \$$

$1125 \$$

1126

$1127 \$$

$1128 \$$

$1129 \$$

$1130 \$ !$

$1131 \$$ ! Now... Append/get rid of enbackground dvm 5/93

$1132 \$$ if enback_hc then append 'enback_file' 'peak_file'

$1133 \$$ delete 'enback_file'

$1134 \$$

$1135 \$$

$1136 \$$

$1137 \$$

$1138 \$$

$1139 \$$

say "'"

say " System is now doing an enback using background"

say " "+bold_on+"backgnd'+bold_off+"

enback_qual="/negative/header=nd_ams_headers:enback.hdr"

if .not. neg_areas then -

enback_qual="/nonegative/header-nd_ams_headers:enback.hdr"

enback'enback_qual"enback_line'/bkg=bkg: ALPHA0 !rsh, added : for ln

type 'enback_file'

$1140 \$$ !

$1141 \$$

$1142 \$ !$ 
Appendix 2.B Listing of DCL file NDAL PROCESS.COM. file as of January 10, 1996

$1143 \$$ ! dvm 5/93 changed to not generate report unless asked, also printout

$1144 \$$ ! moved to later on to put in proper order

$1145 \$$

$1146 \$$

$1147 \$$

if postnid_hc

$1148 \$$

then

$1149 \$$

say "'"

$1150 "$

say -

$1151 \$$

$1152 \$$

$1153 \$$ !

$1154 \$$

$1155 \$$

$1156 \$$

$1157 \$$

1158 \$!generate spectral data plot dvm 5/93

$1159 \$$

$1160 \$$

$1161 \$$

$1162 \$$

$1163 \$$

System is now doing a post-nid peak on "+bold_on+"'aconfig"'+bold_off+"

ndreport/nid=apeak'nidpeak_line' ALPHA0

type 'nidpeak_file'

endif

$1164 \$$

$1165 \$$

$1166 \$$

$1167 \$$

$1168 \$$

$1169 \$$

$1170 \$ !$

$1171 \$$

$1172 \$$

$1173 \$$

$1174 \$$

$1175 \$$

$1176 \$$

$1177 \$$

$1178 \$$

$1179 \$$

1180

$1181 \$$

$1182 \$$

$1183 \$$

area/nolist/symbol=backcounts/start $=3$ alpha0

if plot_queue .eqs. "TT:" then goto skip_spec_plot

if plot_hc .and. plot_queue .nes. "'

then

if dvm

then

say "'"

say" System is now developing a plot on "+bold_on+"Alpha0"+bold_off+"

plot/alpha/left $=3500 /$ right $=6500 /$ nonid/noregion ALPHA0 'plot_file' else

area/nolist/symbol=backcounts/start=3 alpha0

if (backcounts .eqs. "0 0")

then

say "'"

say " Spectrum contains 0 counts and will not be plotted"

ask reply " Hit RETURN to continue..."

goto SKIP_SPEC_PLOT

endif

1184 \$skip_spec_plot:

$1185 \$$

$1186 \$$

$1187 \$$

$1188 \$$ say ""

display nd_ams_archive_xchar':'aconfig' -

/linear/proto $=$ 'plot_proto"plot_line'/land $/$ start $=3$

endif !dvm

endif ! he flag

!-.. 
Appendix 2.B Listing of DCL file NDAL_PROCESS.COM, file as of January 10, 1996

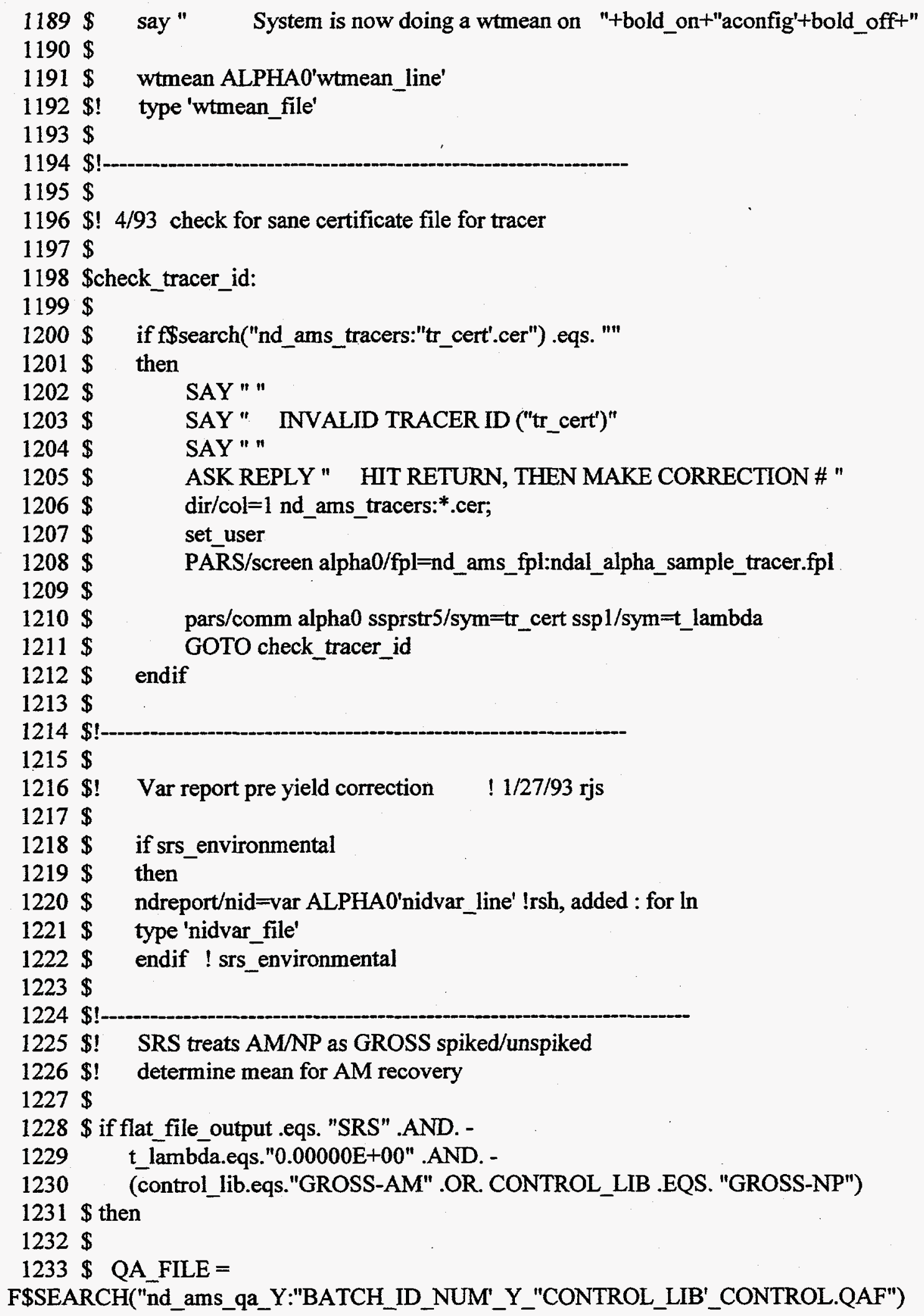


Appendix 2.B Listing of DCL file NDAL PROCESS.COM, file as of January 10, 1996

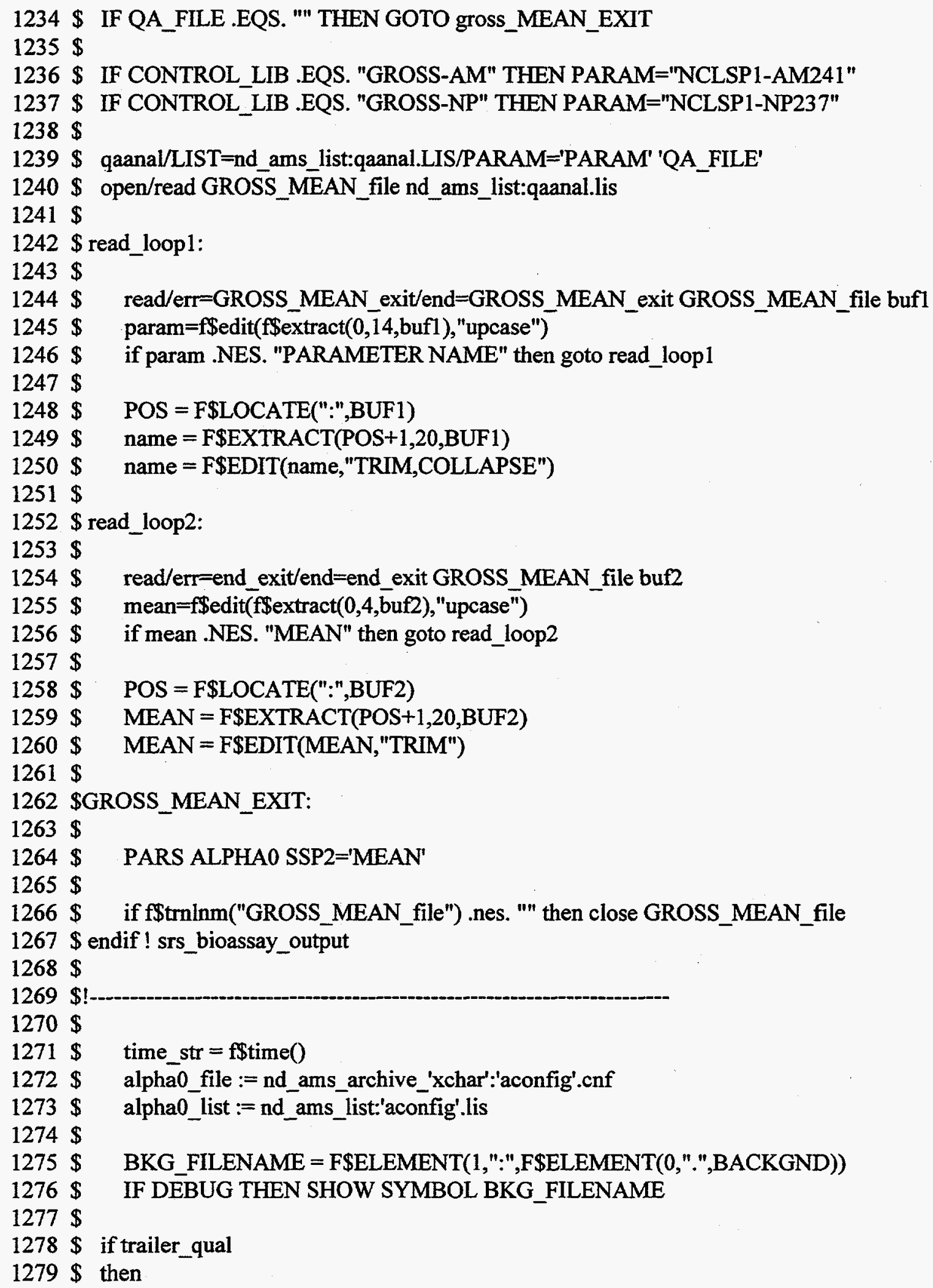


Appendix 2.B Listing of DCL file NDAL PROCESS.COM, file as of January 10, 1996

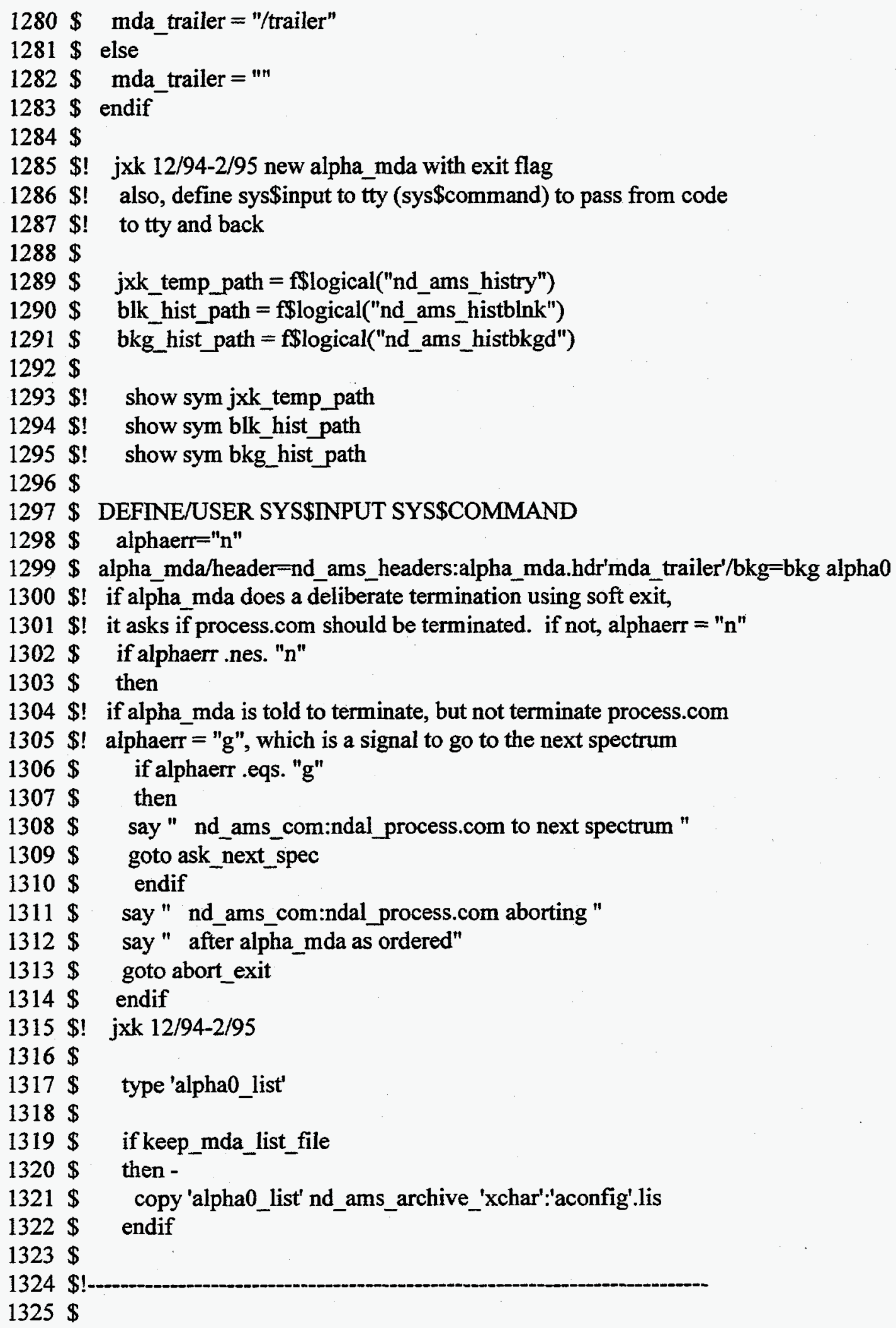


Appendix 2.B Listing of DCL file NDAL_PROCESS.COM, file as of January 10, 1996

$1326 \$$ ! dvm $5 / 93$ report printouts moved to here and set in order

$1327 \$$

1328 \$skip_alpha_mda:

$1329 \$$

1330 \$ QAXFER ALPHA0 nd_ams_qa_y:'batch_id_num'_y_'CONTROL_LIB'_matrix_type' $1331 \$$

1332 \$QAXFER ALPHA0 nd_ams_qa_y:batch_y_control_lib'_matrix_type'

$1333 \$$

$1334 \$ !$

$1335 \$$

$1336 \$$ ! SRS treats AMNP as GROSS spiked/unspiked

$1337 \$$

1338 \$ if flat_file_output .eqs. "SRS" .AND. -

1339 t_lambda .nes. "0.00000E+00" .AND. -

1340 (control_lib .eqs. "GROSS-AM" .OR. CONTROL_LIB .EQS. "GROSS-NP")

$1341 \$$ then

1342 IF f $\$$ search ("nd_ams_qa_y:"batch_id_num'y_"CONTROL_LIB'_control.qaf; *") .nes. "' 1343 \$ THEN

1344 \$ qaxfer alpha0 nd_ams_qa_y:'batch_id_num'_y_CONTROL_LIB'_control.qaf

1345 endif ! srs_bioassay_output

1346 \$ endif! srs_bioassay_output

$1347 \$$

$1348 \$$

$1349 \$$

1350 if flat_file_output.NES. "NO"

1351 \$ then

$1352 \$$

1353 \$ set def nd_ams_biotrack_bif:

1354 \$ DEFINE/USER SYS\$INPUT SYS\$COMMAND

$1355 \$$ ! show sym jxk_temp_path

$1356 \$$

$1357 \$ !$ jxk 8/9/95 the code flat_file_output used to be called y12.for

1358 \$ RUN ND_AMS_EXE:'FLAT_FILE_OUTPUT

$1359 \$$

$1360 \$$ SET DEF ND_AMS_USER:

$1361 \$$

1362 endif ! flat_file_output

$1363 \$$

$1364 \$$ if xchar eqs. "C" then goto CONTROL ! CONTROLS

$1365 \$$ if xchar .eqs. "R" then goto REAGENT ! REAGENTS

$1366 \$$ goto clean_up ! SAMPLES

$1367 \$$

$1368 \$$

$1369 \$$

1370 control: ! controls

$1371 \$$ 


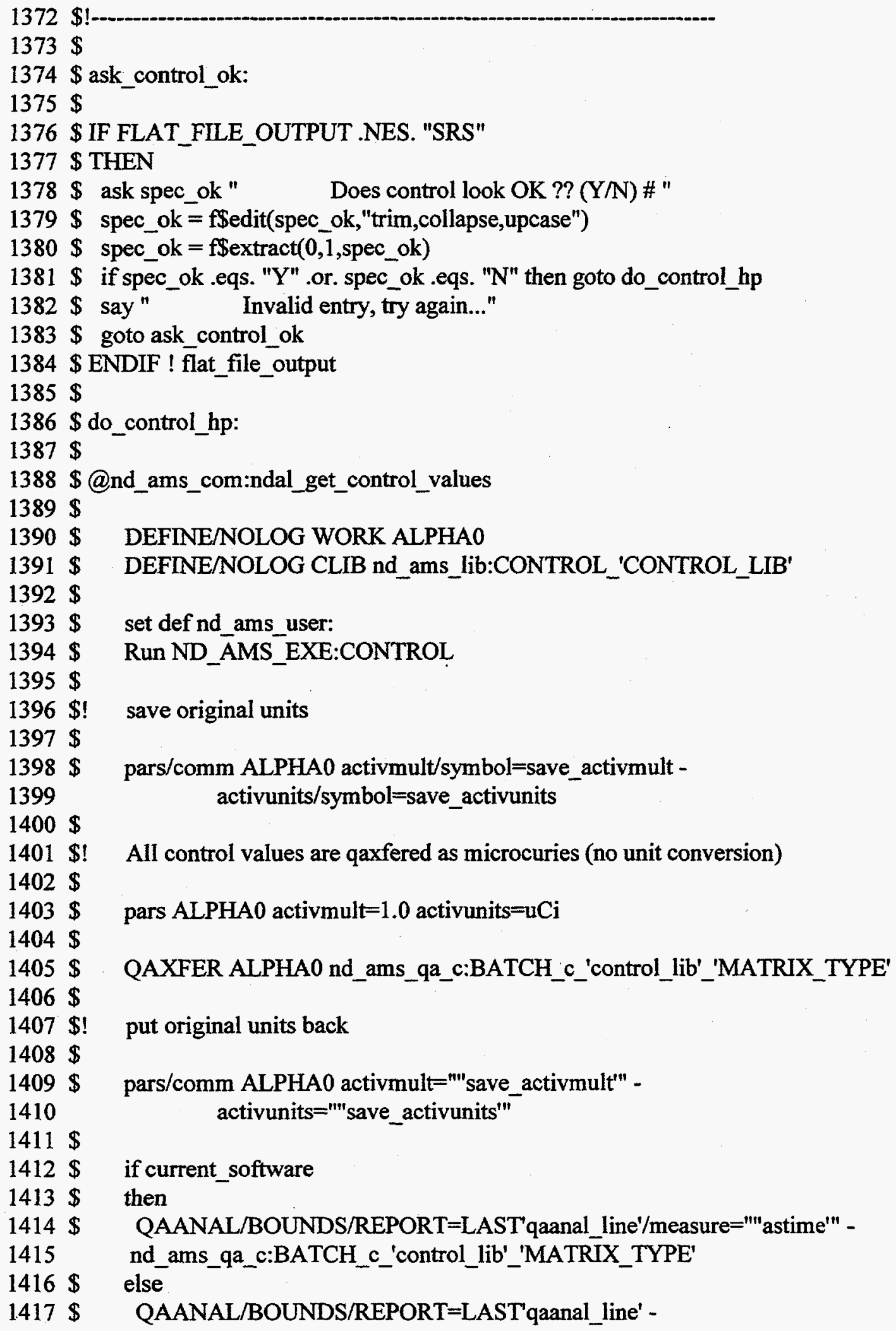




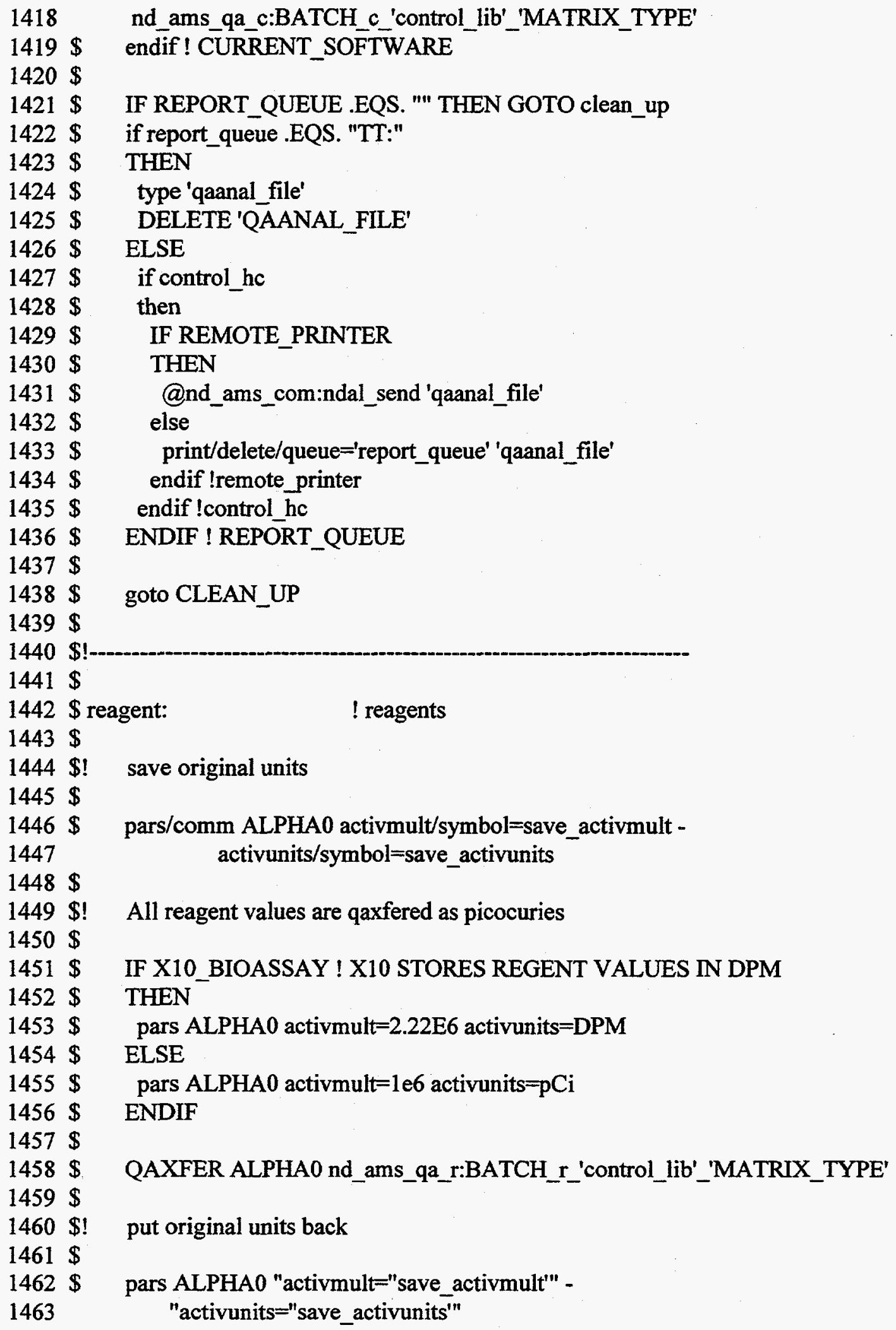


Appendix 2.B Listing of DCL file NDAL PROCESS.COM, file as of January 10. 1996

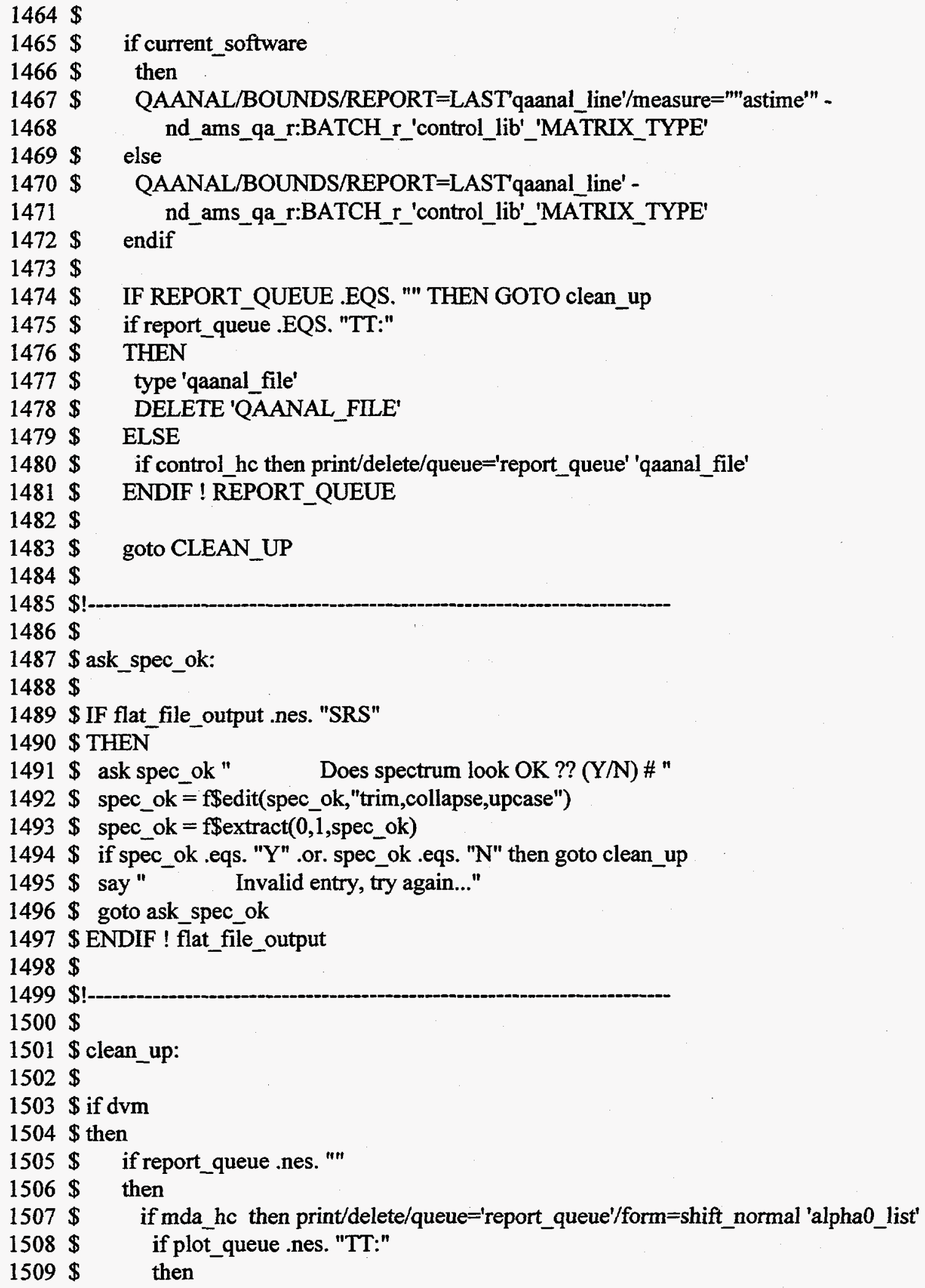




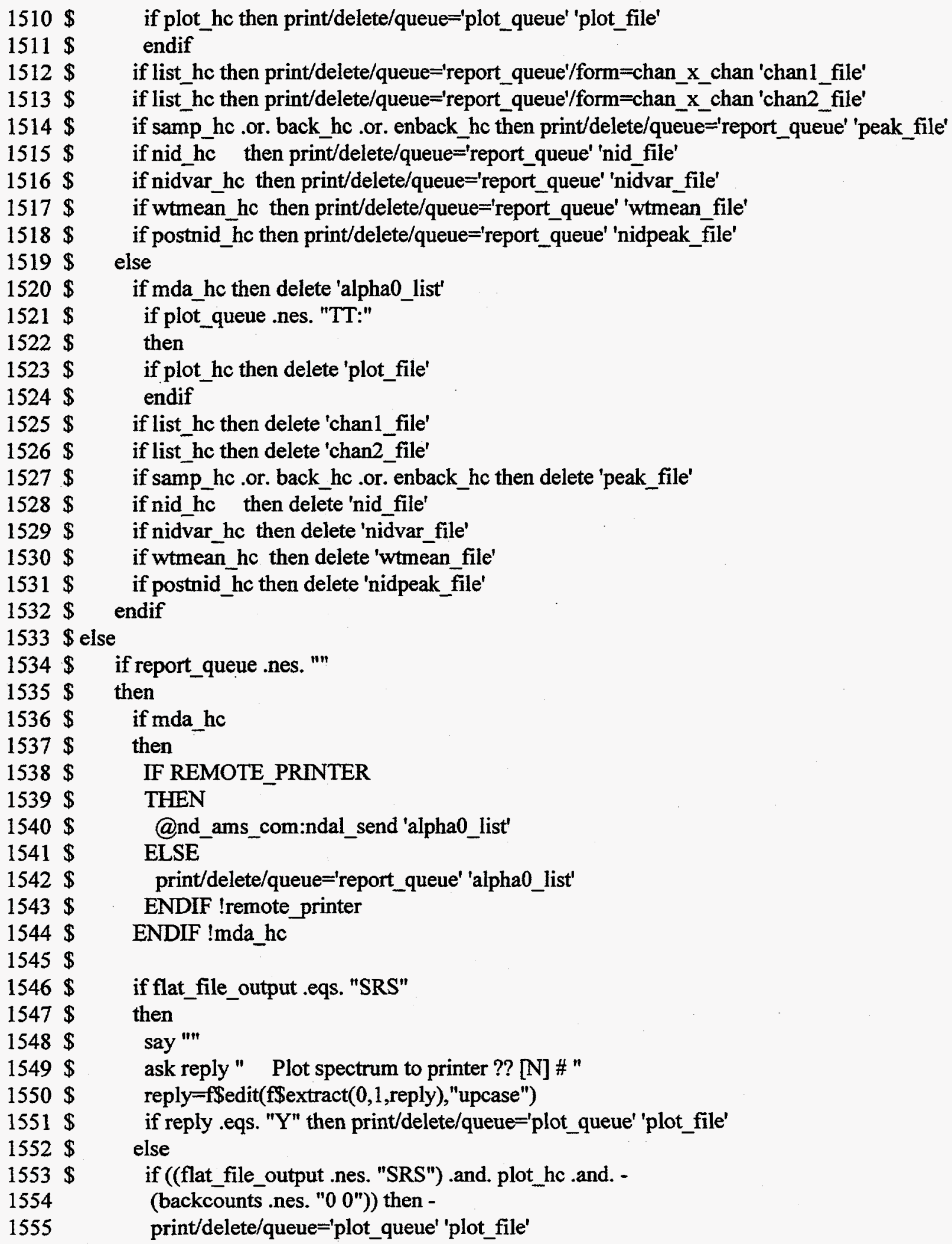


Appendix 2.B Listing of DCL file NDAL_PROCESS.COM. file as of January 10, 1996

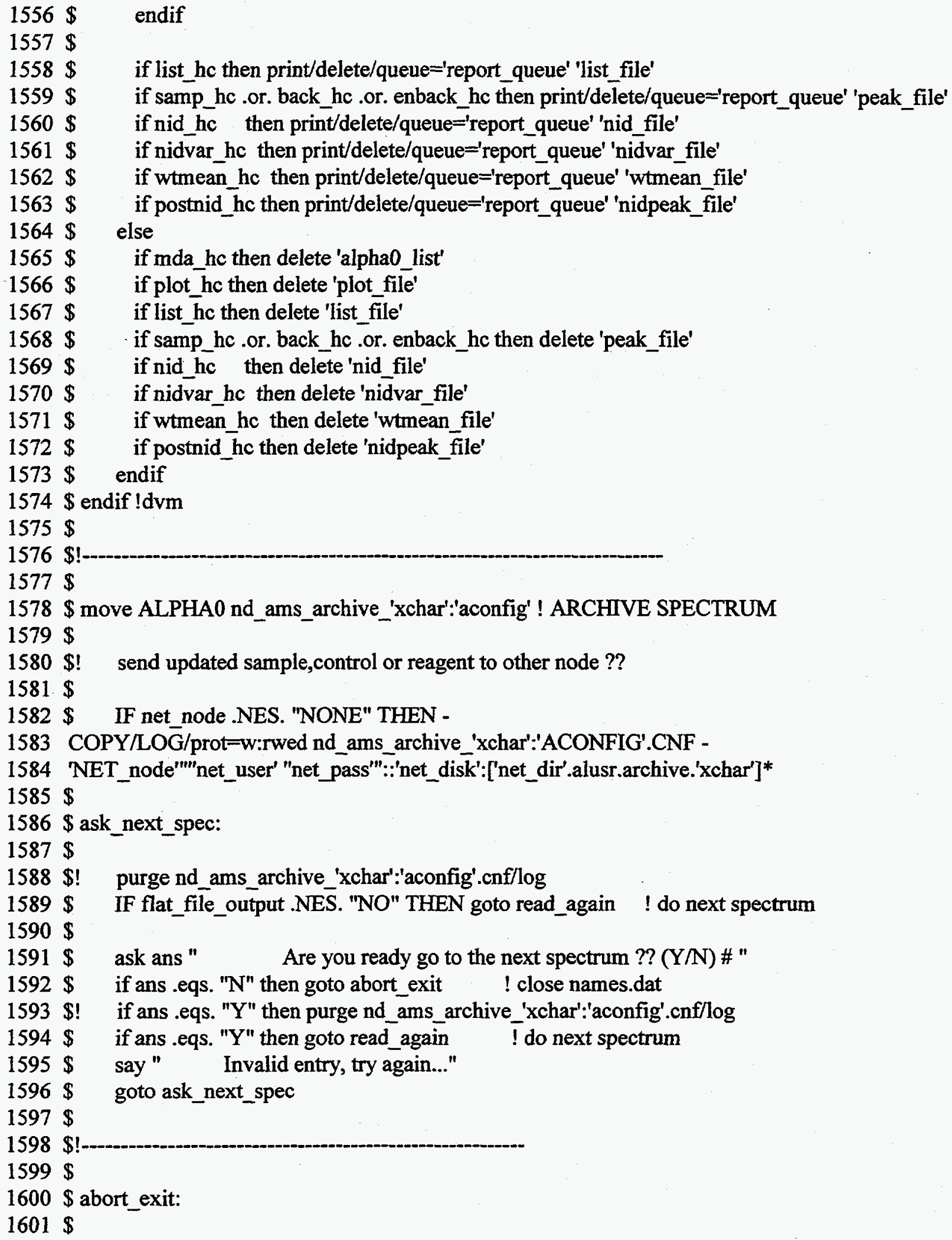




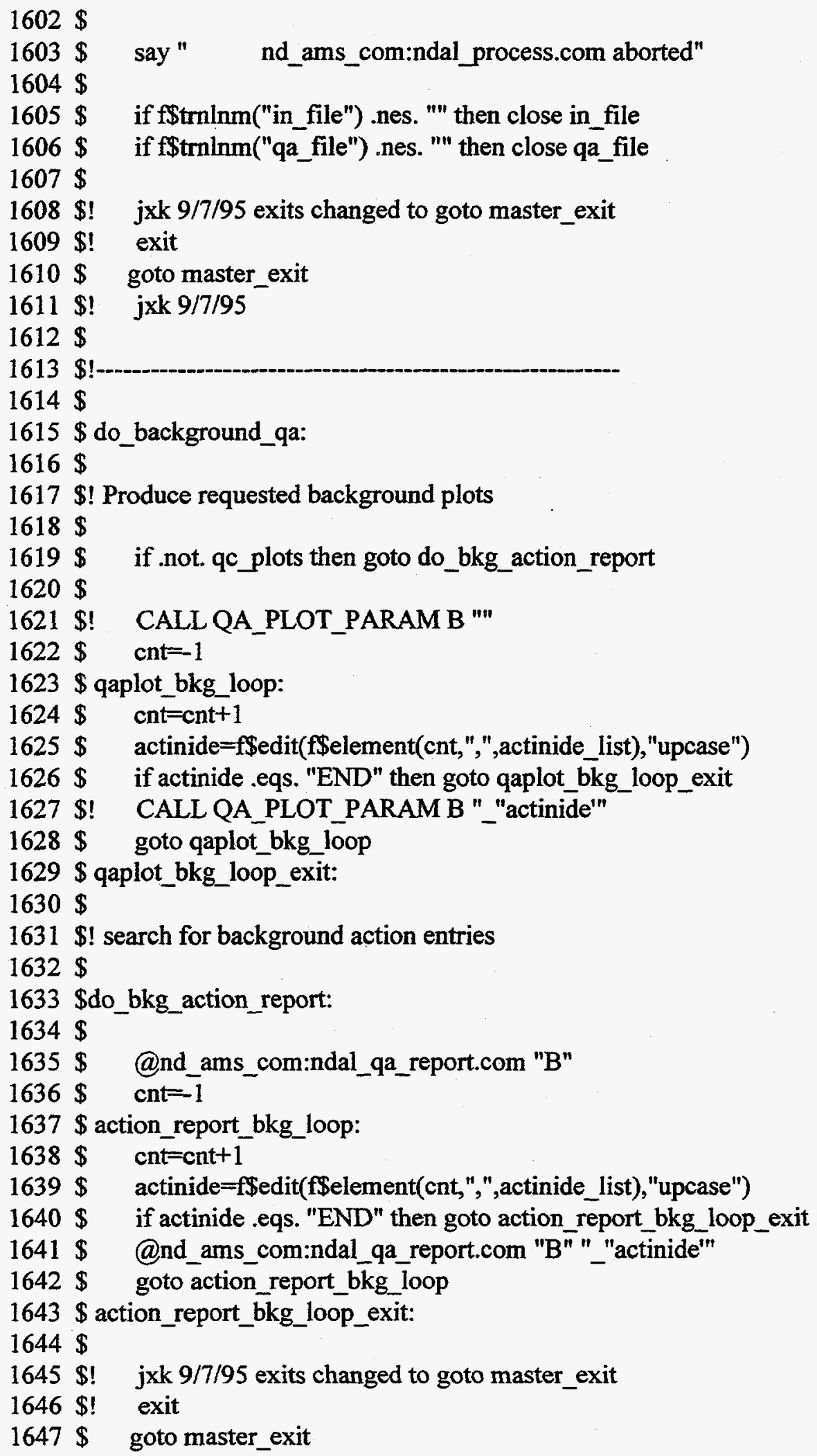


$1648 \$$ j jxk $9 / 7 / 95$

$1649 \$$

$1650 \$ !$

$1651 \$$

1652 \$do_daily_qa:

$1653 \$$

$1654 \$$ ! Produce requested daily plots

$1655 \$$

$1656 \$$ if .not. qc_plots then goto do_daily_action_report

$1657 \$$

$1658 \$$ ! CALL qa_plot_param "D"

$1659 \$$

$1660 \$$ ! search for daily action entries

$1661 \$$

1662 \$do_daily_action_report:

$1663 \$$

$1664 \$$ @nd_ams_com:ndal_qa_REPORT "D"

$1665 \$$

$1666 \$$ j jxk 9/7/95 exits changed to goto master_exit

$1667 \$ ! \quad$ exit

$1668 \$$ goto master_exit

$1669 \$$ ! jxk $9 / 7 / 95$

$1670 \$$

$1671 \$ !$

$1672 \$$

1673 \$do_weekly_qa:

$1674 \$$

$1675 \$$ if calsum_hc

$1676 \$$ then

$1677 \$$ time_str $=$ f $\$$ time()

1678 \$ RUN ND_AMS_EXE:CALIBRATION_REPORT

$1679 \$$ TYPE ND_AMS_LIST:calibration_report.lis

$1680 \$$

1681 \$ IF REPORT_QUEUE .EQS. "" THEN GOTO SKIP_CALIB_REPORT

$1682 \$$ if report_queue.EQS. "TT:"

$1683 \$$ THEN

$1684 \$$ DELETE ND_AMS_LIST:calibration_report.lis;*

1685 \& ELSE

$1686 \$$ IF REMOTE_PRINTER

$1687 \$$ THEN

$1688 \$ @ N D \_A M S \_C O M: N D A L \_S E N D$ nd_ams_list:calibration_report.lis

$1689 \$$ else

$1690 \$$ print/delete/queue='report_queue' nd_ams_list:calibration_report.lis

$1691 \$$ endif !remote_printer

1692 \$ ENDIF !REPORT_QUEUE

1693 \$ ENDIF !CALSUM_HC 


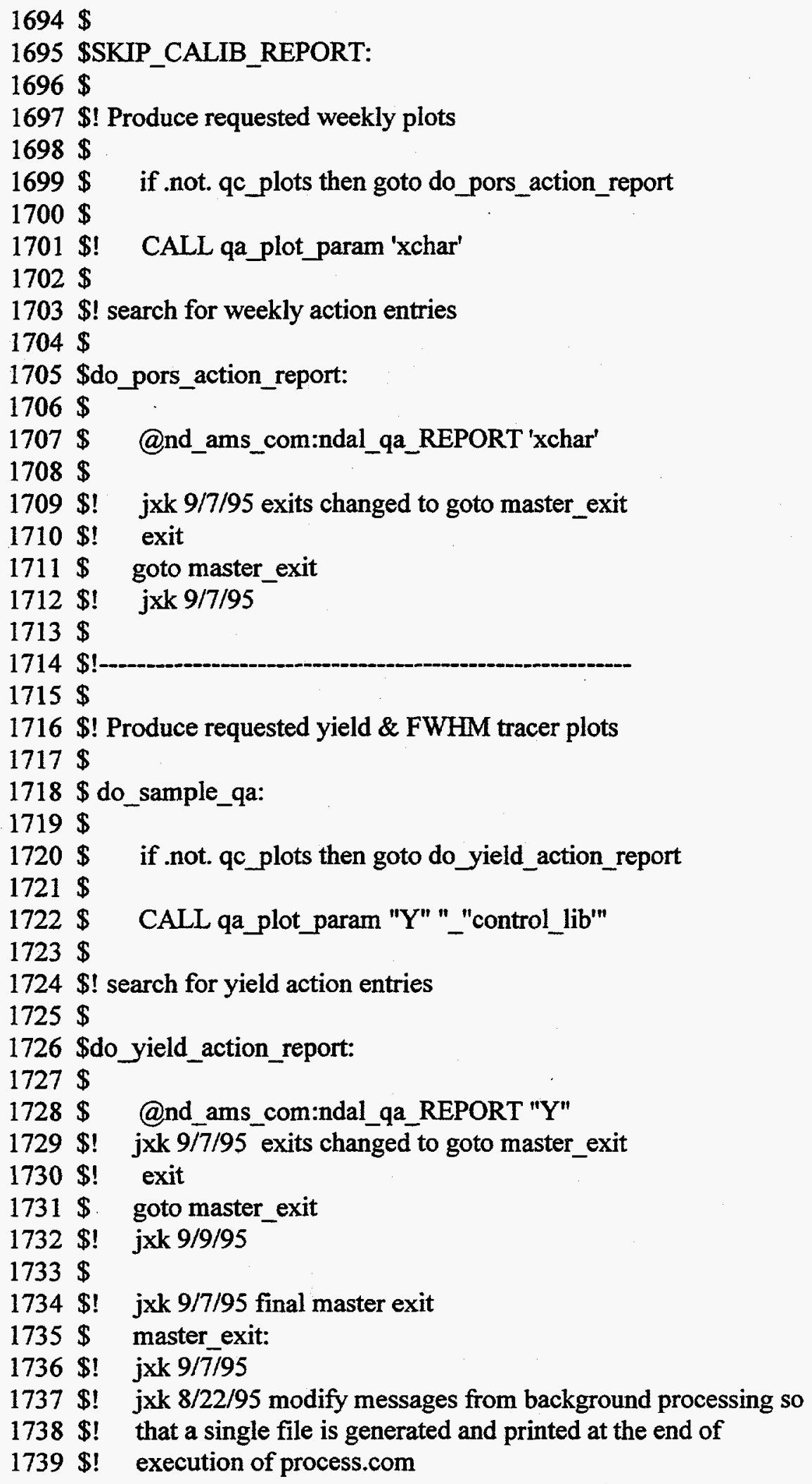


$1740 \$$ ! close the messages file, print, and delete

$1741 \$$ if $\$$ edit( $\$$ extract $(0,1, y 12$ bioassay), "upcase") .eqs. "Y" -

1742 .or. $\mathbf{\$} \$$ edit(f\$extract $(0,1$, new_reagent_calc),"upcase") .eqs. "Y"

$1743 \$$ then

$1744 \$$ ! close jxknotefile

$1745 \$$ ! temporary type of messages file to see contents

1746 \$ type 'jxk_temp_notemsg'

$1747 \$$ ! count lines in the message file to see if it has any messages

$1748 \$$ jxklinecnt=0

$1749 \$$ open/read jxkinfile 'jxk_temp_notemsg'

$1750 \$$ ! 3 reads to make sure I count past header lines

$1751 \$$ read/end_of_file=jxknoteeof jxkinfile jxkline

$1752 \$$ jxklinecnt $=\overline{\text { jxklinecnt }+1}$

$1753 \$$ read/end_of_file=jxknoteeof jxkinfile jxkline

$1754 \$$ jxklinecnt $=\bar{j}$ xklinecnt +1

$1755 \$$ read/end_of_file=jxknoteeof jxkinfile jxkline

$1756 \$$ jxklinecnt $=\bar{j}$ jklinecnt +1

$1757 \$$

$1758 \$$

$1759 \$$

jxknoteeof:

$1760 \$ !$ if there is more than the header line(s), print messages file

$1761 \$ !$ and delete

$1762 \$$

$1763 \$$

$1764 \$$

$1765 \$$

if jxklinecnt .gt. jxknotehdlncnt

then

print 'jxk_temp_notemsg'

$1766 \$$ ! delete the messages file so no excess files will hand around

$1767 \$$ ! to possibly cause confusion as well as clutter up disk

$1768 \$$ jxkcolast $=$ "; *"

$1769 \$$ jxkfdel = "'jxk_temp_notemsg'"+"'+jxkcolast"'

$1770 \$$ ! show sym jxkfdel

$1771 \$$ delete 'jxkfdel'

$1772 \$$ endif ! if y12_bioassay .or. new_reagent_calc

$1773 \$ !$ jxk 8/22/95

$1774 \$$ ! jxk 9/7/95 final master exit

$1775 \$$ exit

$1776 \$$ ! jxk 9/7/95

$1777 \$$

$1778 \$ !$

$1779 \$ ! \mathrm{p} 1$ is the type of analysis, supports prime \& second

$1780 \$ ! 7 / 2 / 92$ cleaned up for prime/second support

$1781 \$$ ! 5/93 looking for nl: on plot device, if so quick exit

$1782 \$$

1783 \$ QA_PLOT_PARAM: SUBROUTINE

$1784 \$$

$1785 \$$ if plot_queue .eqs. "' then exit 
Appendix 2.B Listing of DCL file NDAL PROCESS.COM, file as of January 10, 1996

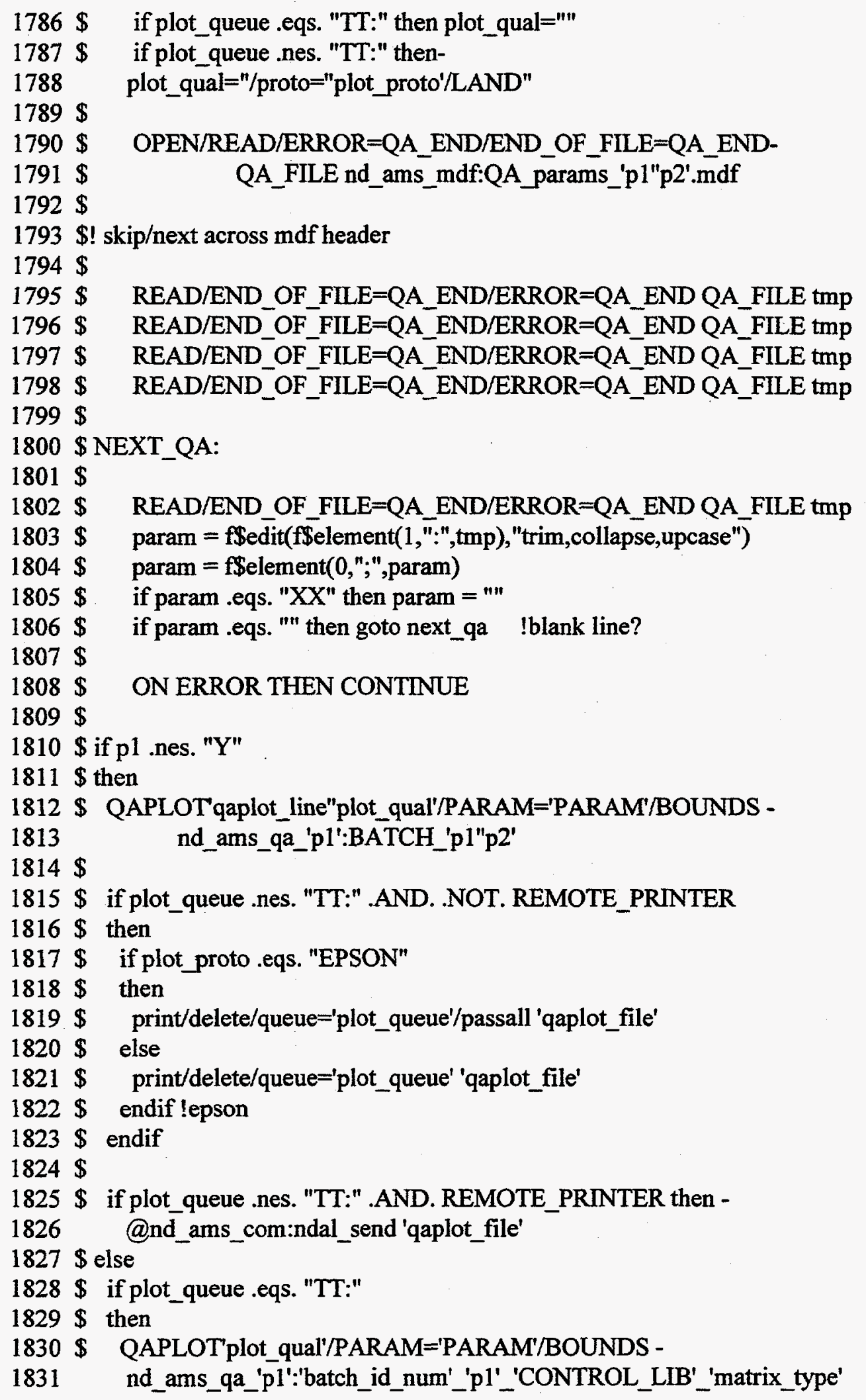


Appendix 2.B Listing of DCL file NDAL PROCESS.COM, file as of January 10, 1996

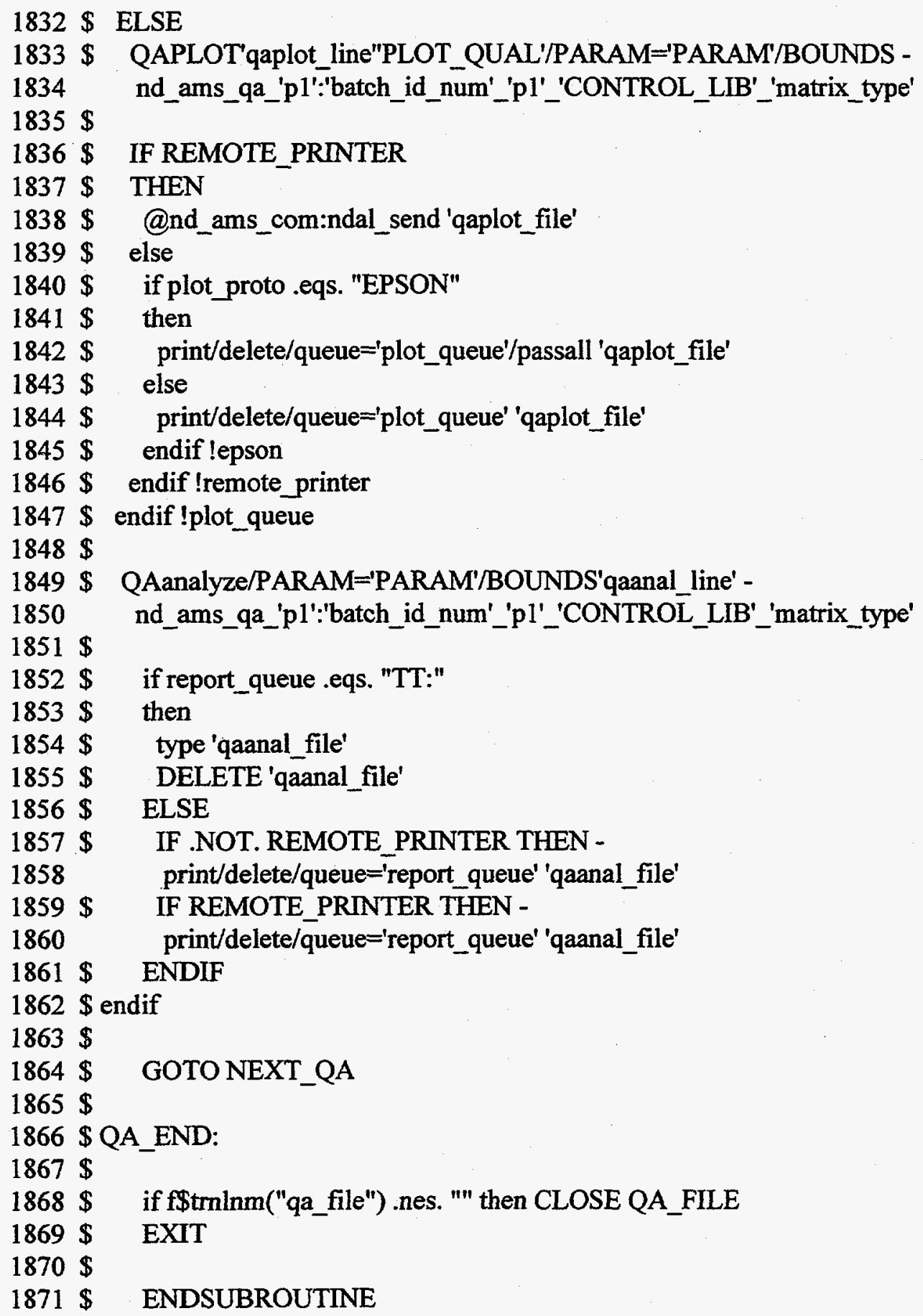




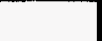




\section{APPENDIX 2.C LISTING OF FORTRAN CODE Y12.FOR, FILE VERSION AS OF NOVEMBER 20, 1995}

File is located on system $B$ disk at dubo:[000000.nd_root.ndamscust.for]

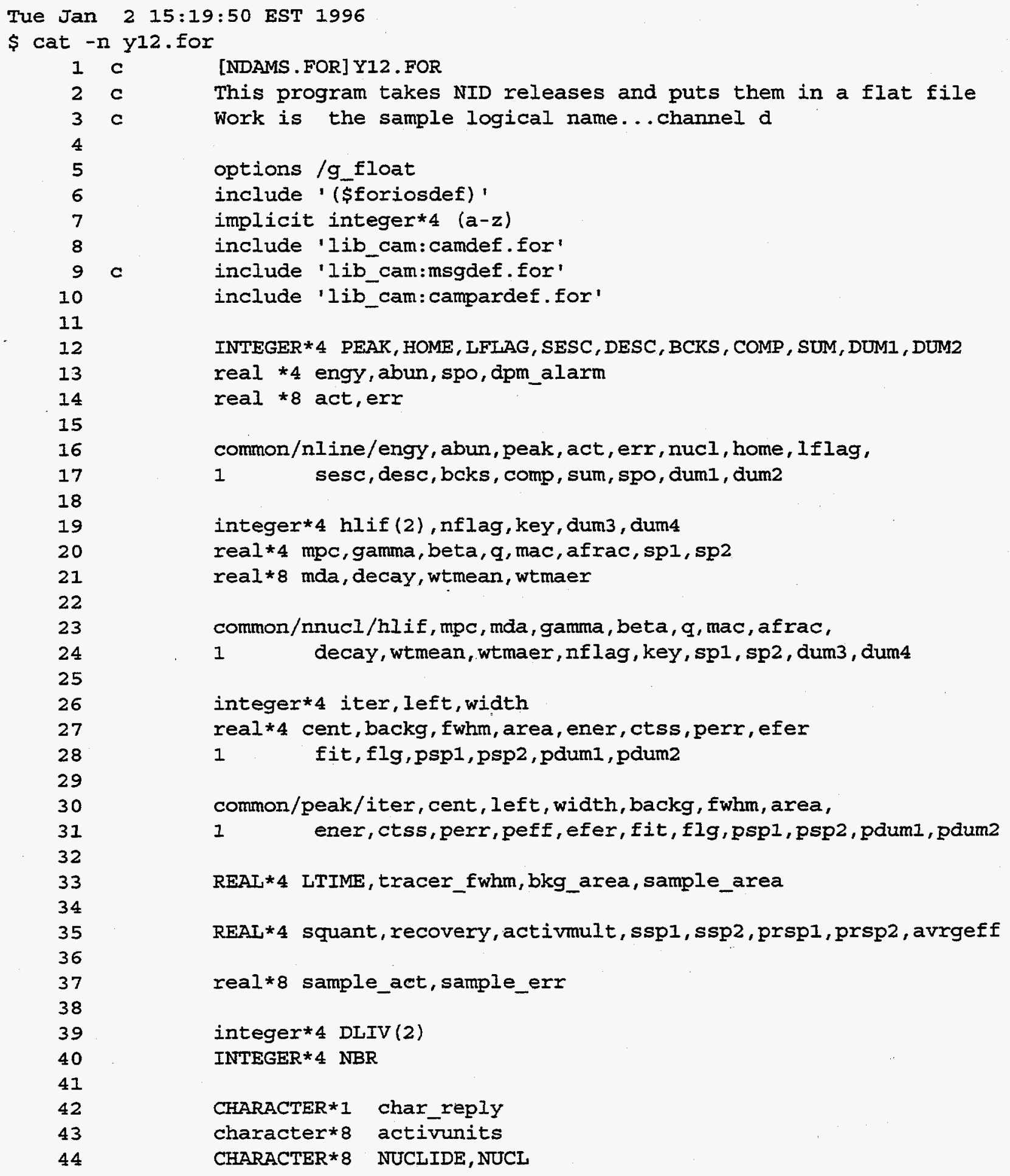


2-122

Appendix 2.C Listing of FORTRAN code Y12.FOR, file version as of November 20.1995

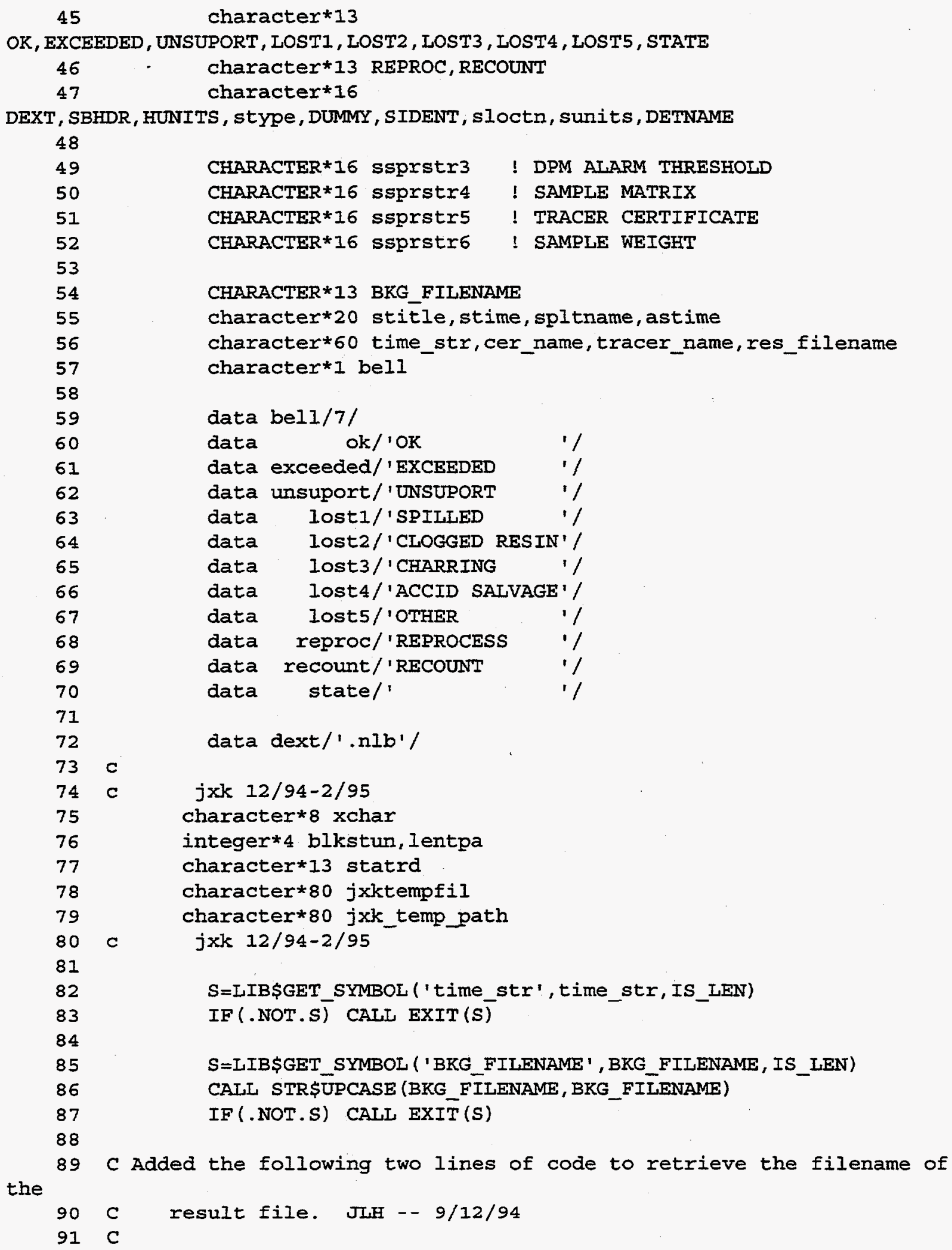


Appendix 2.C Listing of FORTRAN code Y12.FOR, file version as of November 20, 1995

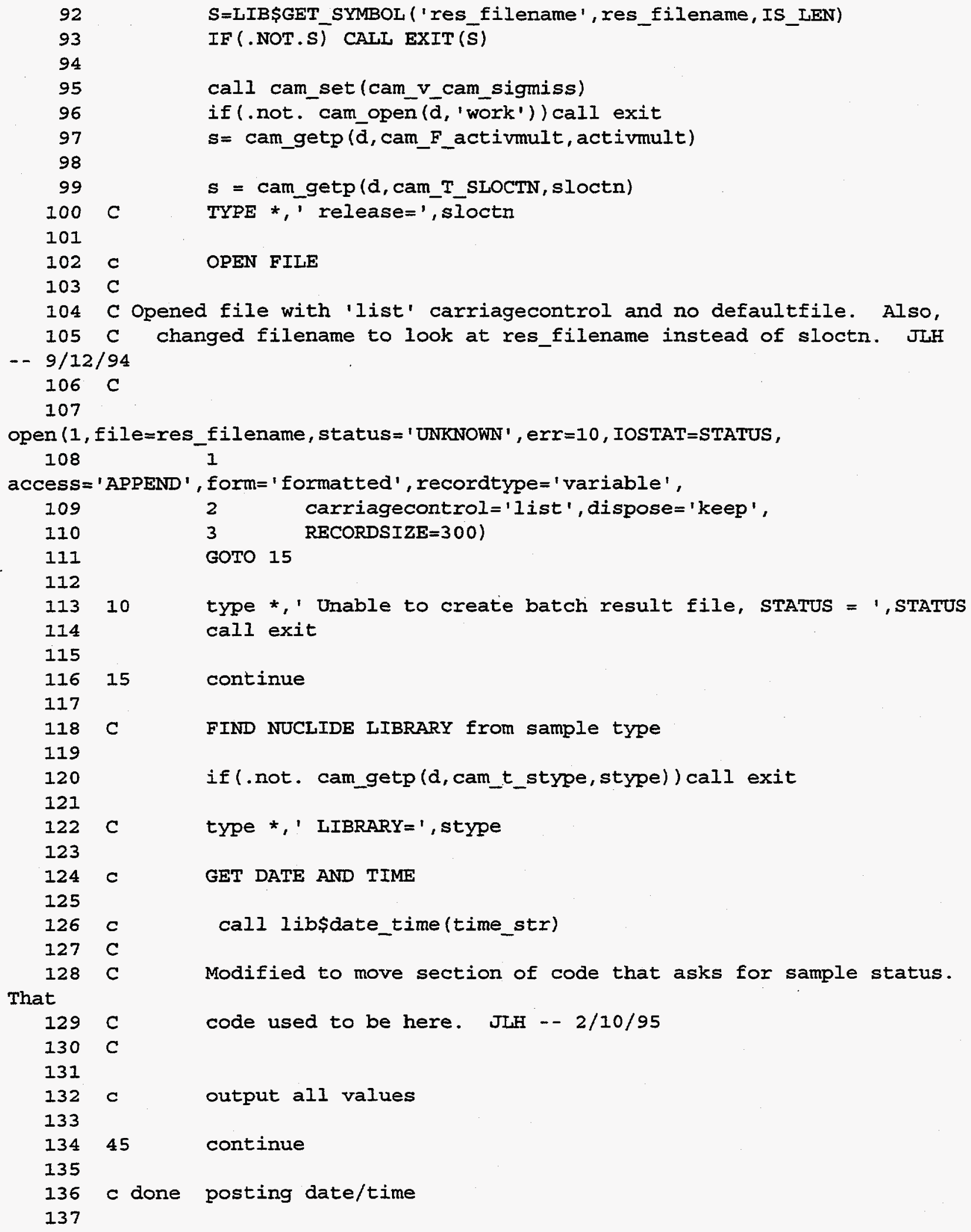


Appendix 2.C Listing of FORTRAN code Y12.FOR, file version as of November 20, 1995

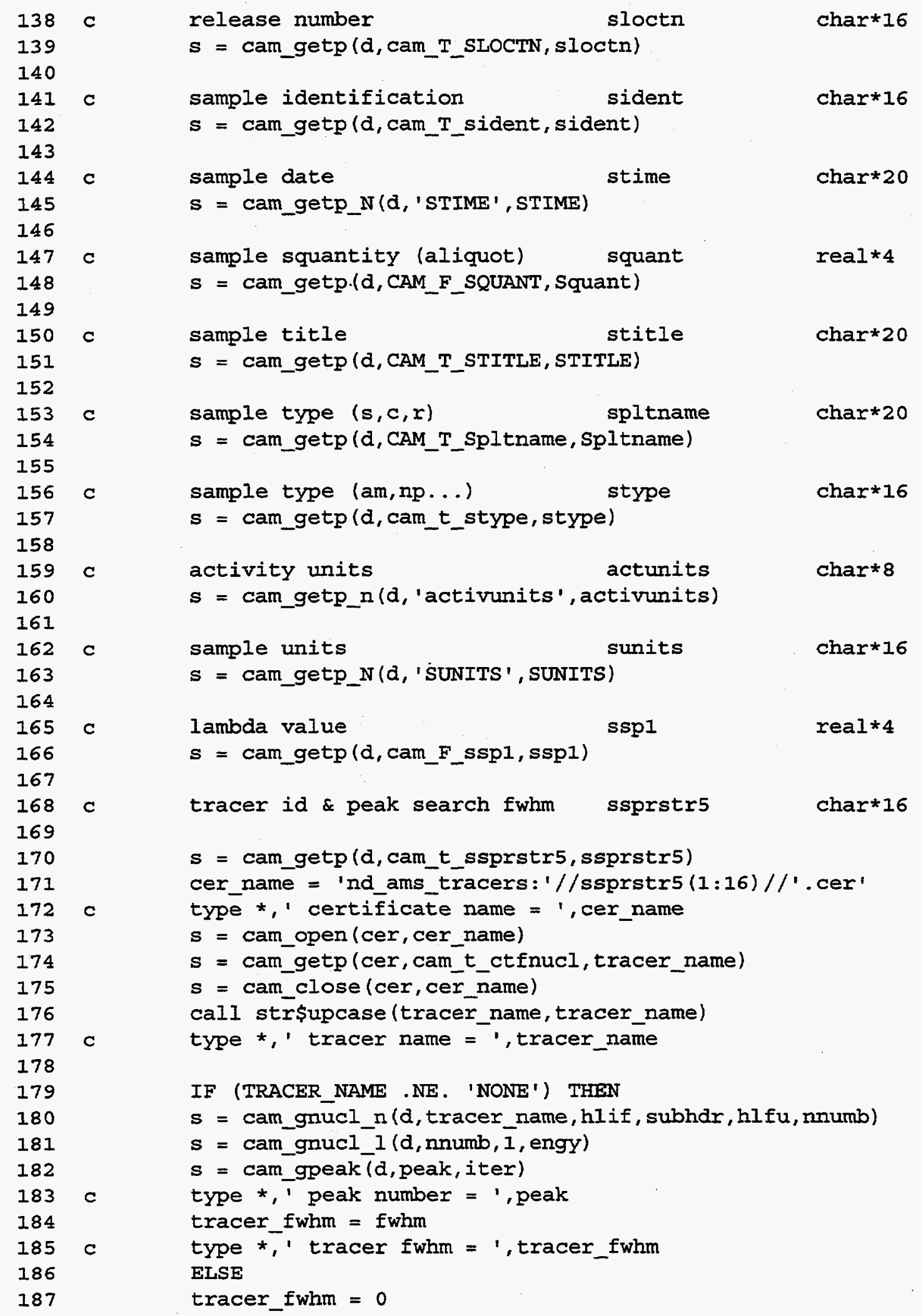


Appendix 2.C Listing of FORTRAN code Y12.FOR, file version as of November 20.1995

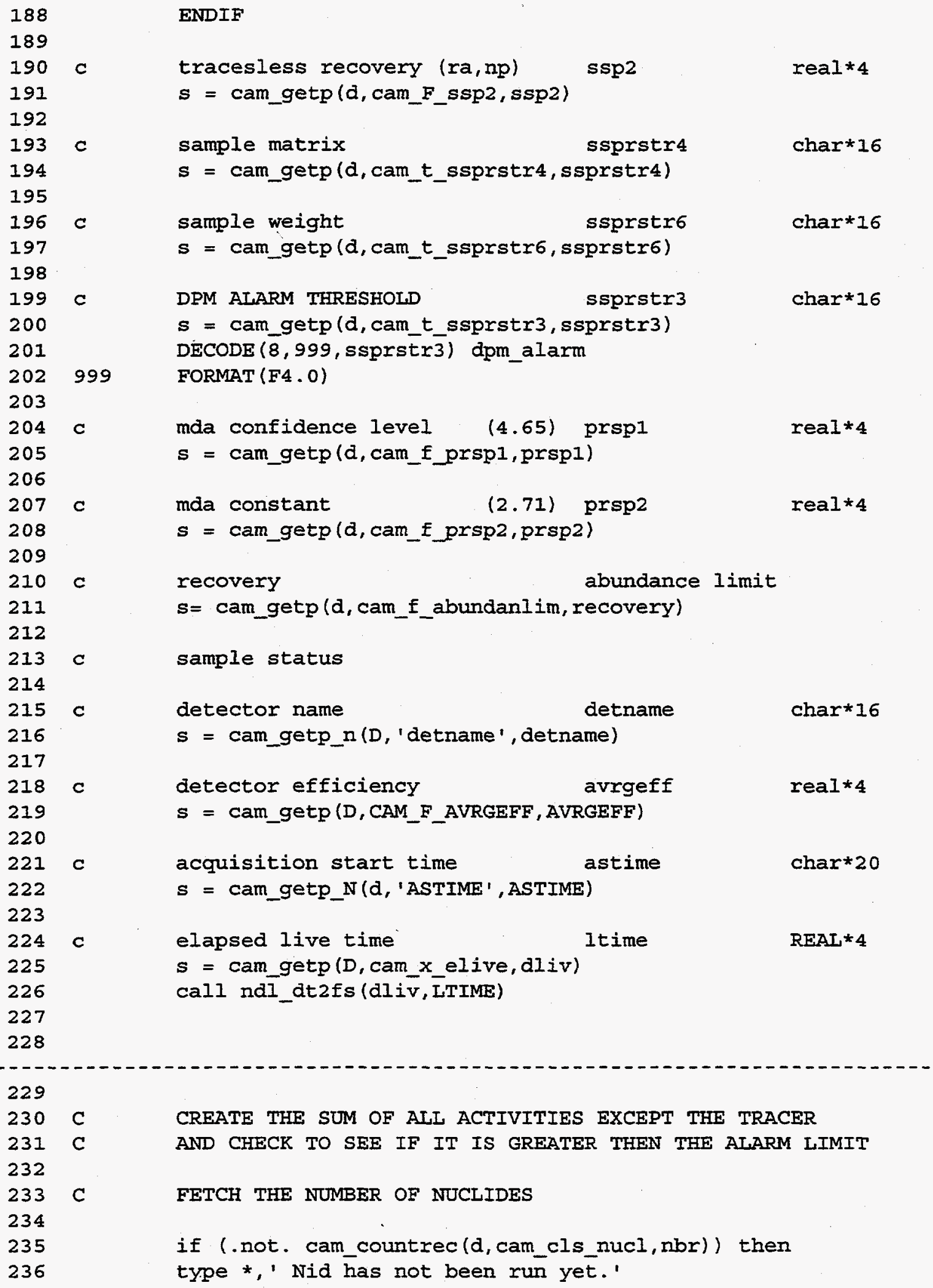


Appendix 2.C Listing of FORTRAN code Y12.FOR, file version as of November 20, 1995

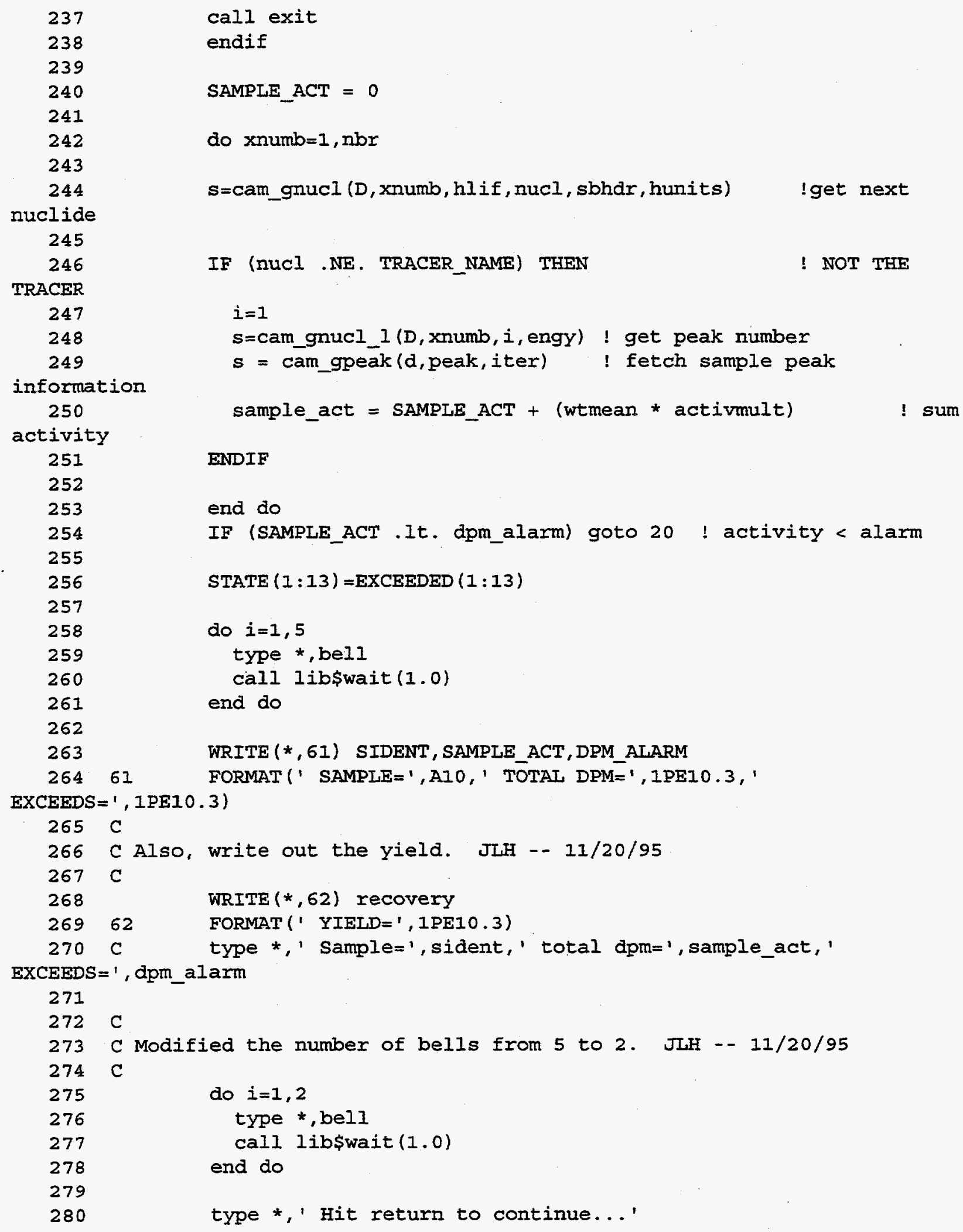


Appendix 2.C Listing of FORTRAN code Y12.FOR, file version as of November 20, 1995

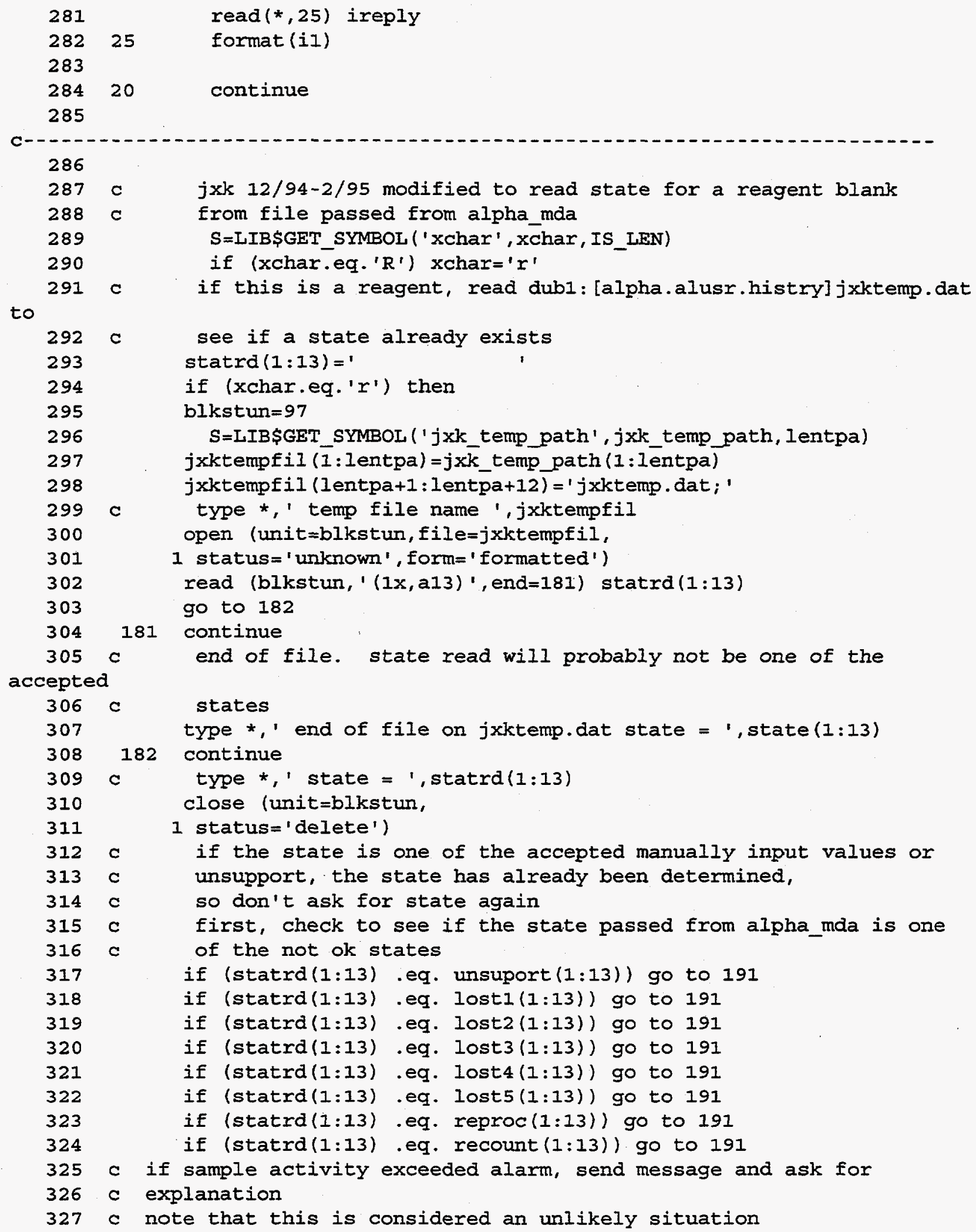


Appendix 2.C Listing of FORTRAN code Y12.FOR, file version as of November 20, 1995

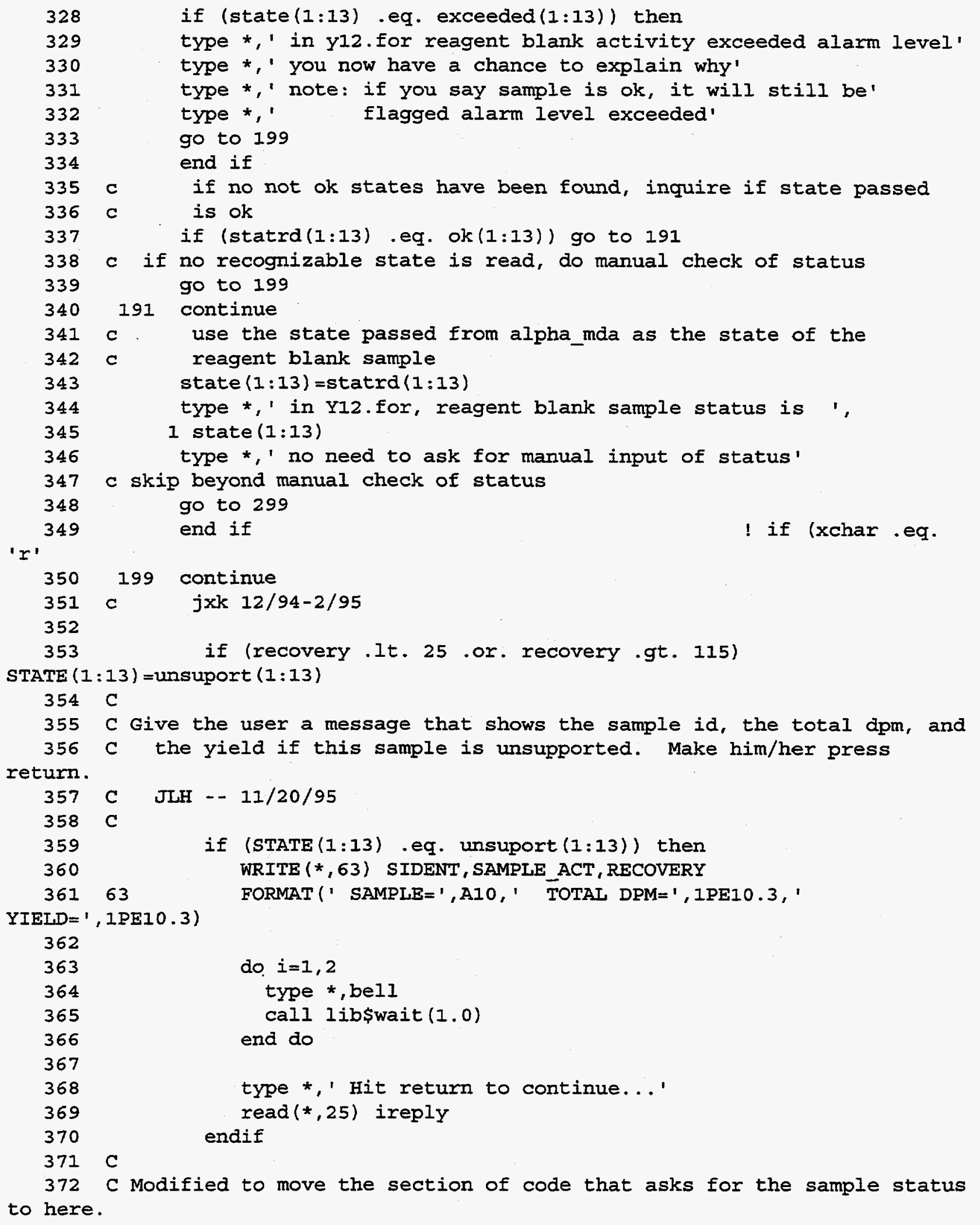


Appendix 2.C Listing of FORTRAN code Y12.FOR, file version as of November 20,1995

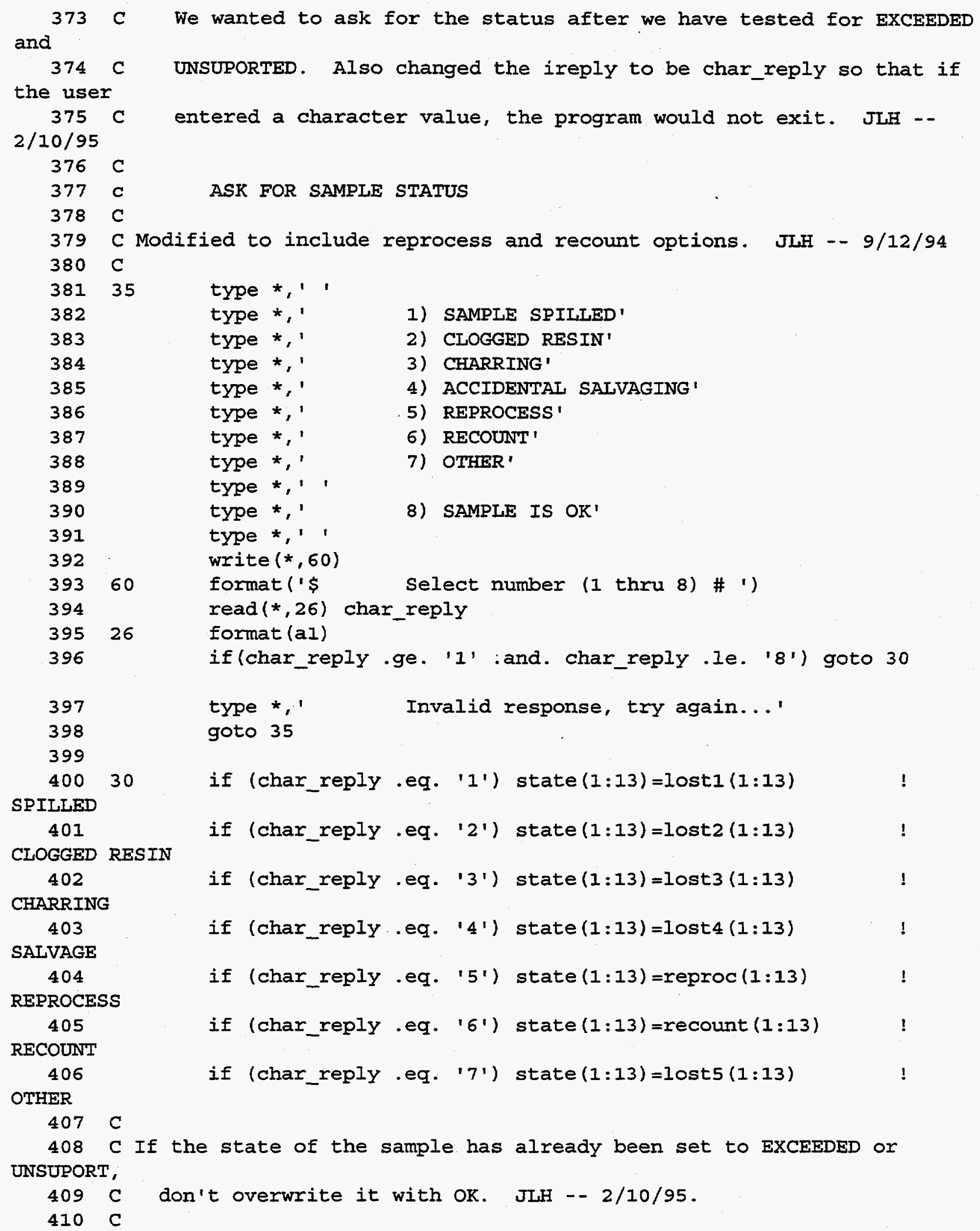


Appendix 2.C Listing of FORTRAN code Y12.FOR, file version as of November 20, 1995

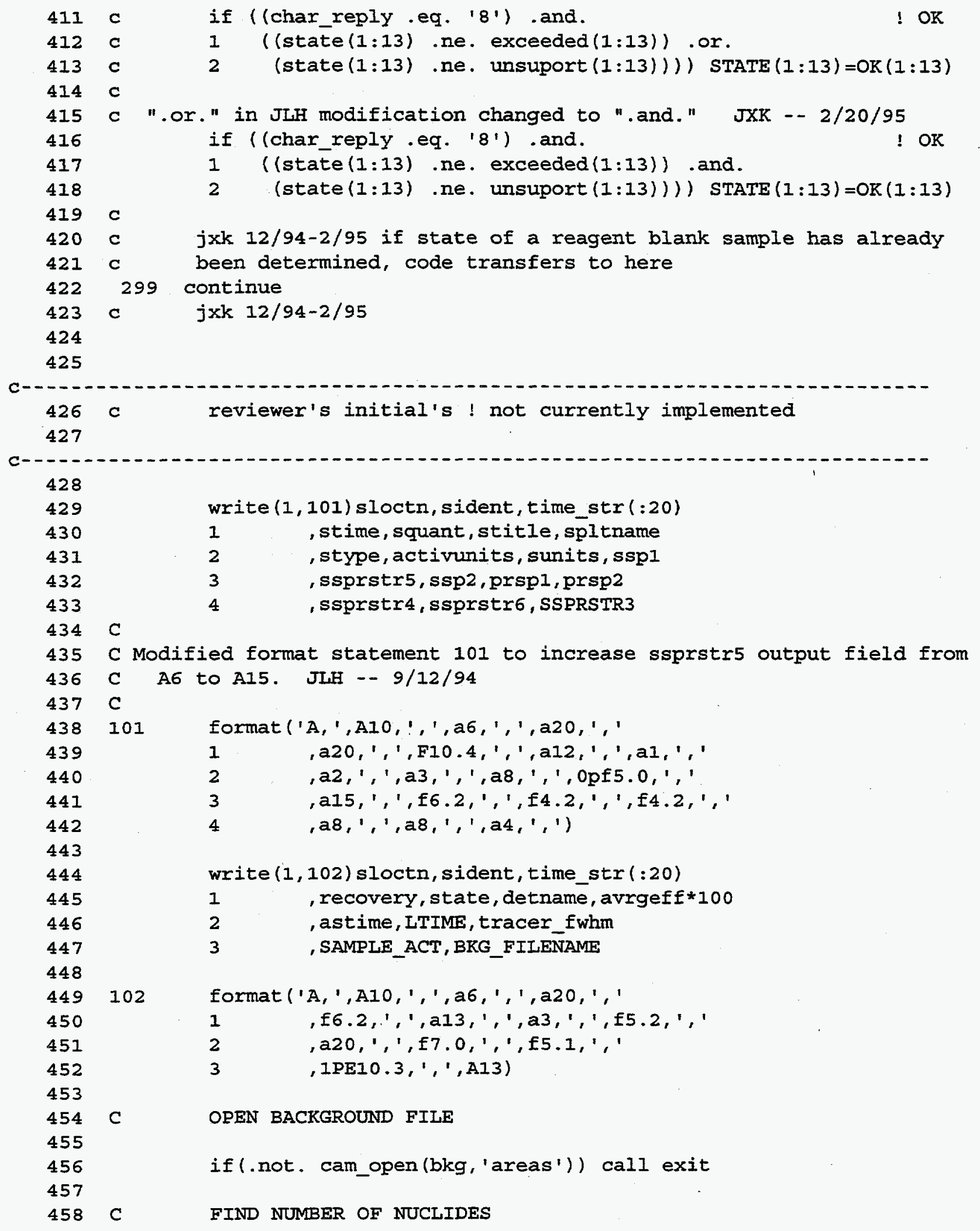




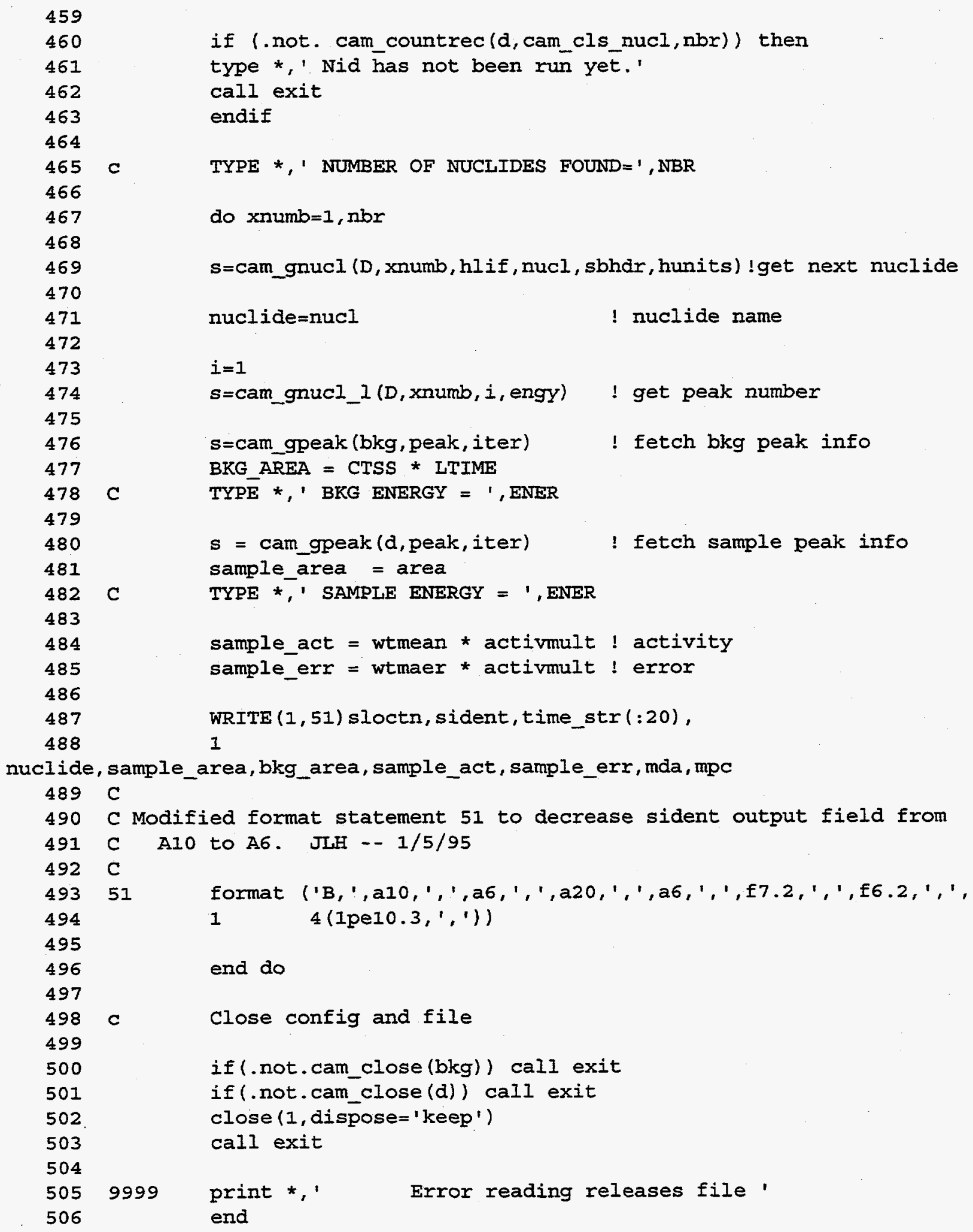




\section{APPENDIX 2.D LISTING OF FORTRAN CODE BKGD_HIST.FOR, FILE VERSION AS OF JANUARY 4, 1996}

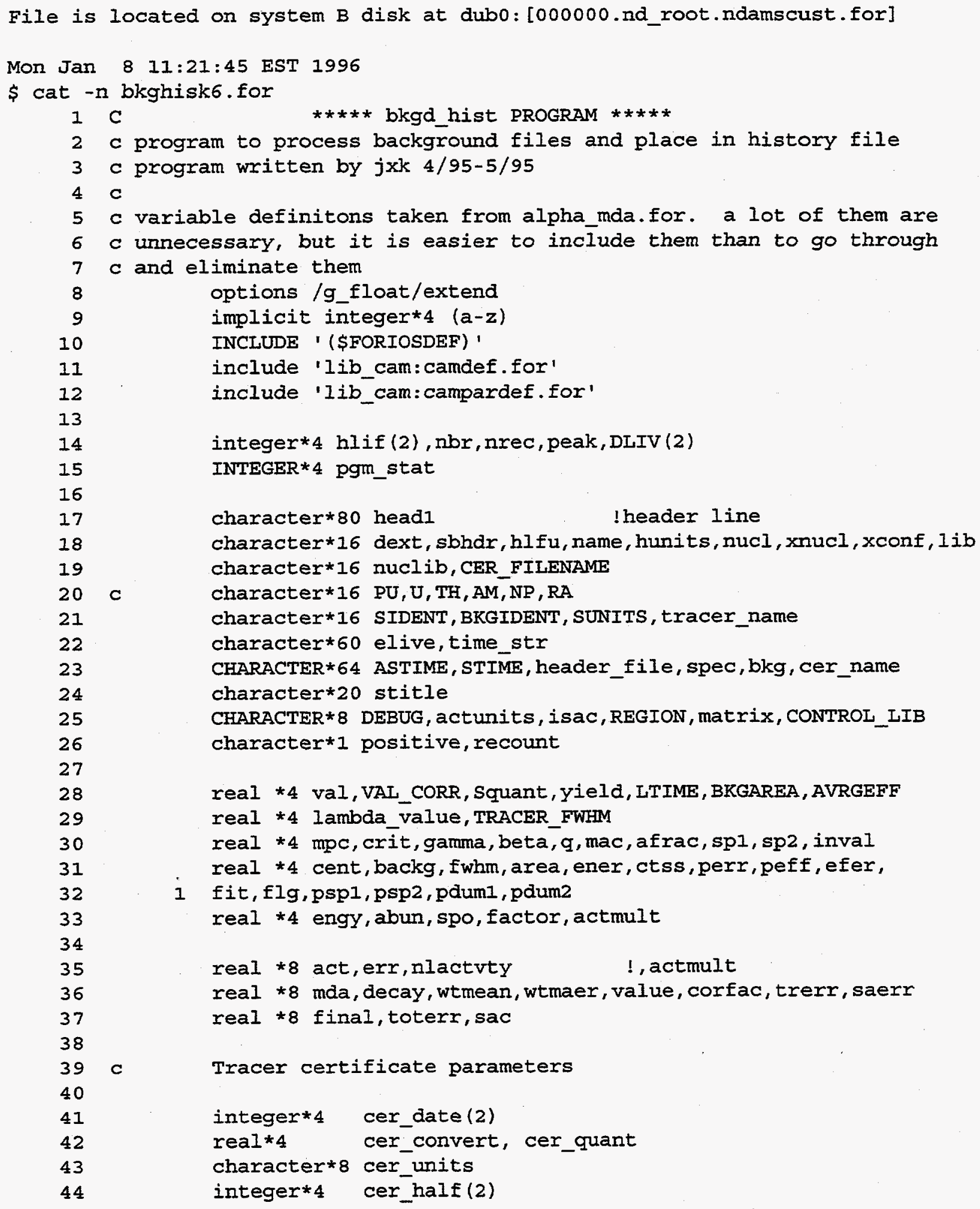


Appendix 2.D Listing of FORTRAN code BKGD HIST.FOR, file version as of January 4.1996

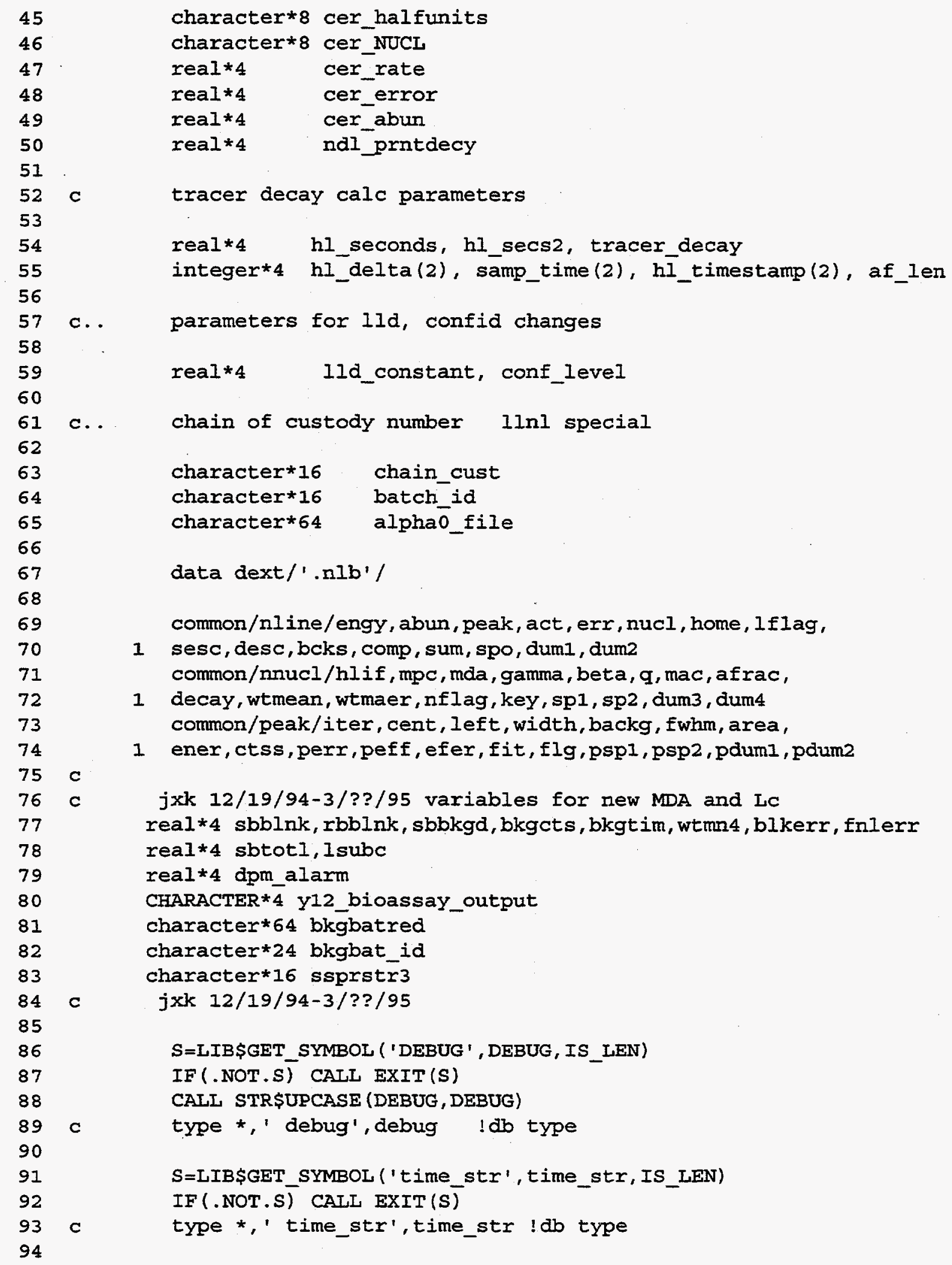


Appendix 2.D Listing of FORTRAN code BKGD_HIST.FOR, file version as of January 4, 1996

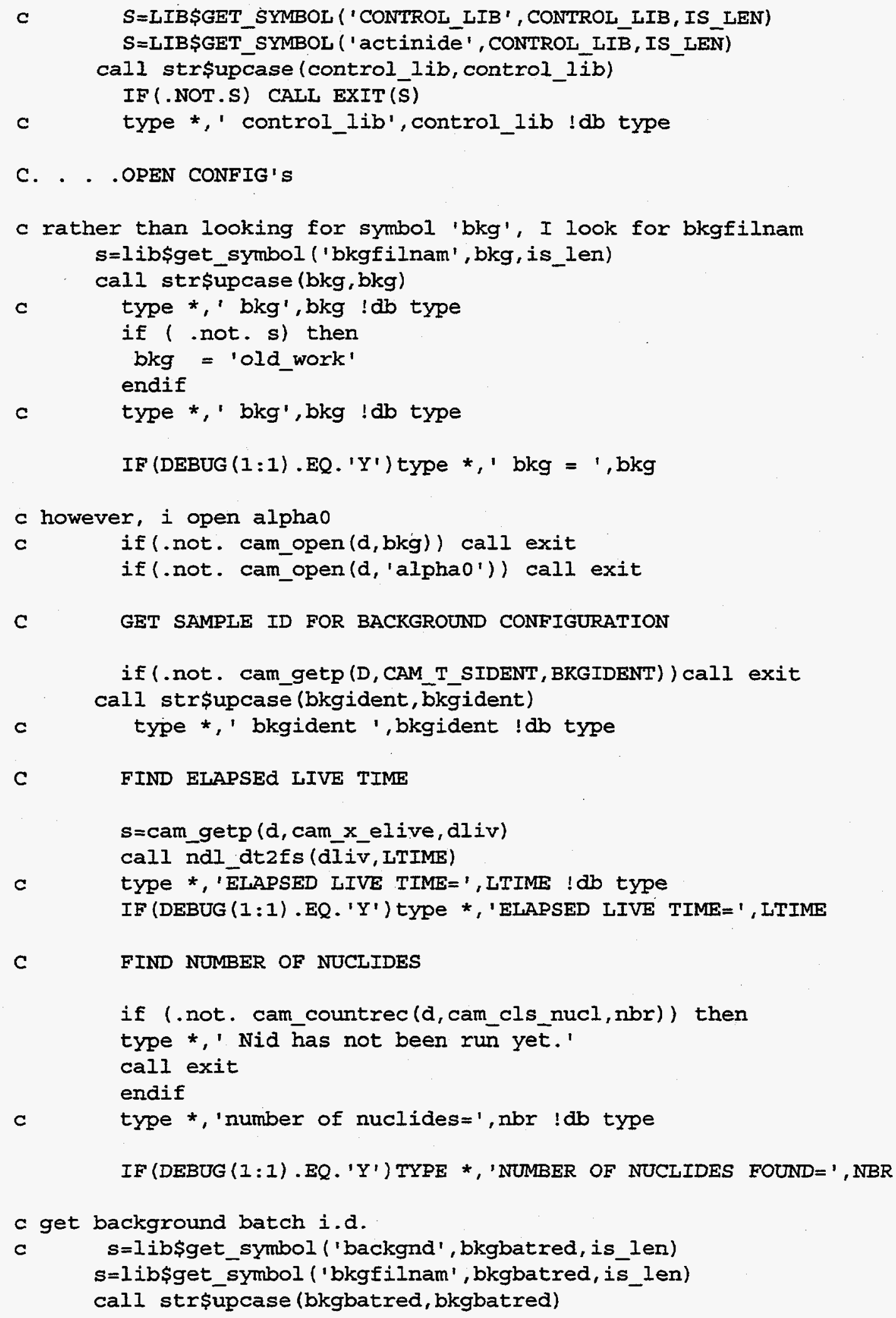


Appendix 2.D Listing of FORTRAN code BKGD HIST.FOR, file version as of January 4, 1996

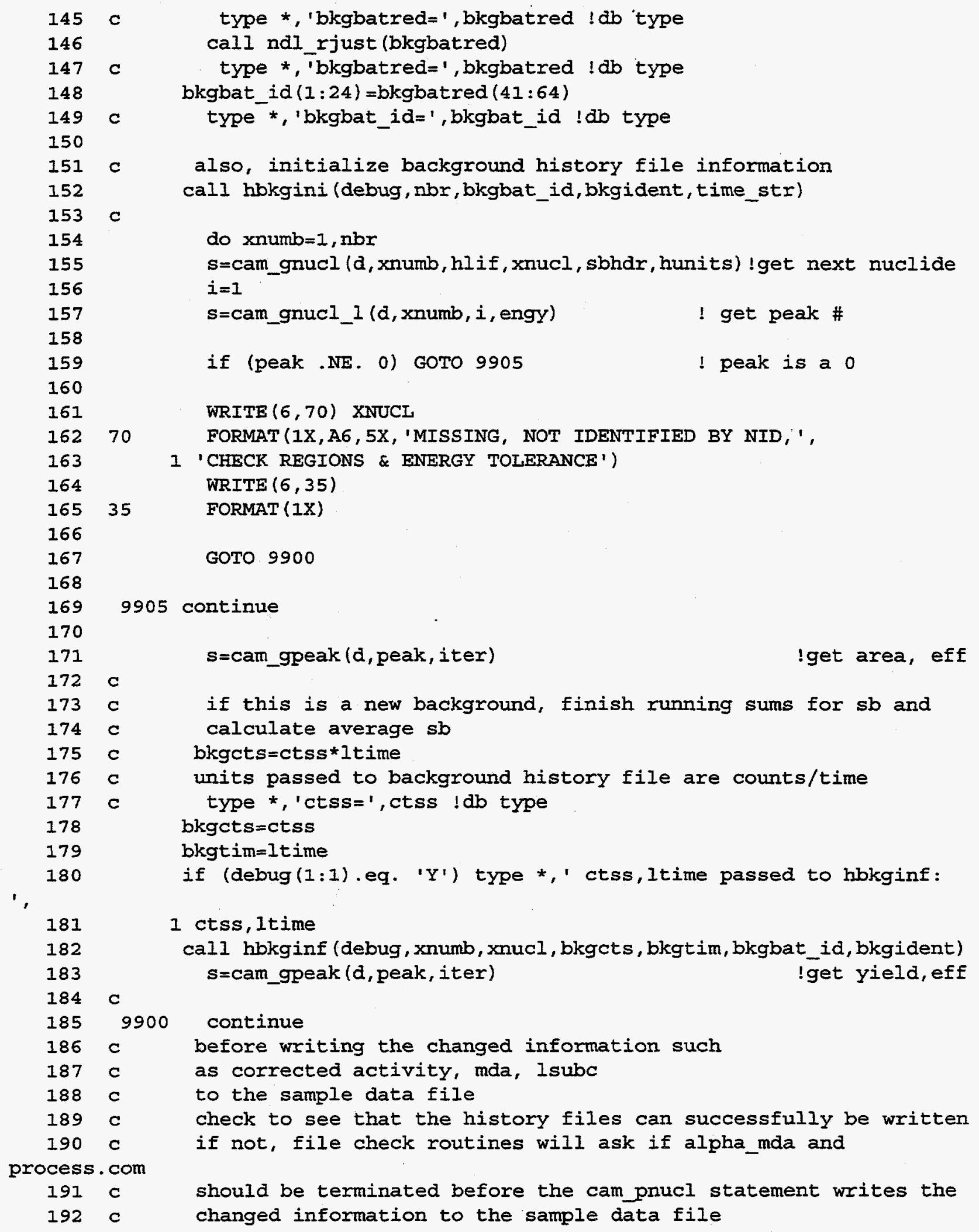


Appendix 2.D Listing of FORTRAN code BKGD_HIST.FOR, file version as of January 4, 1996

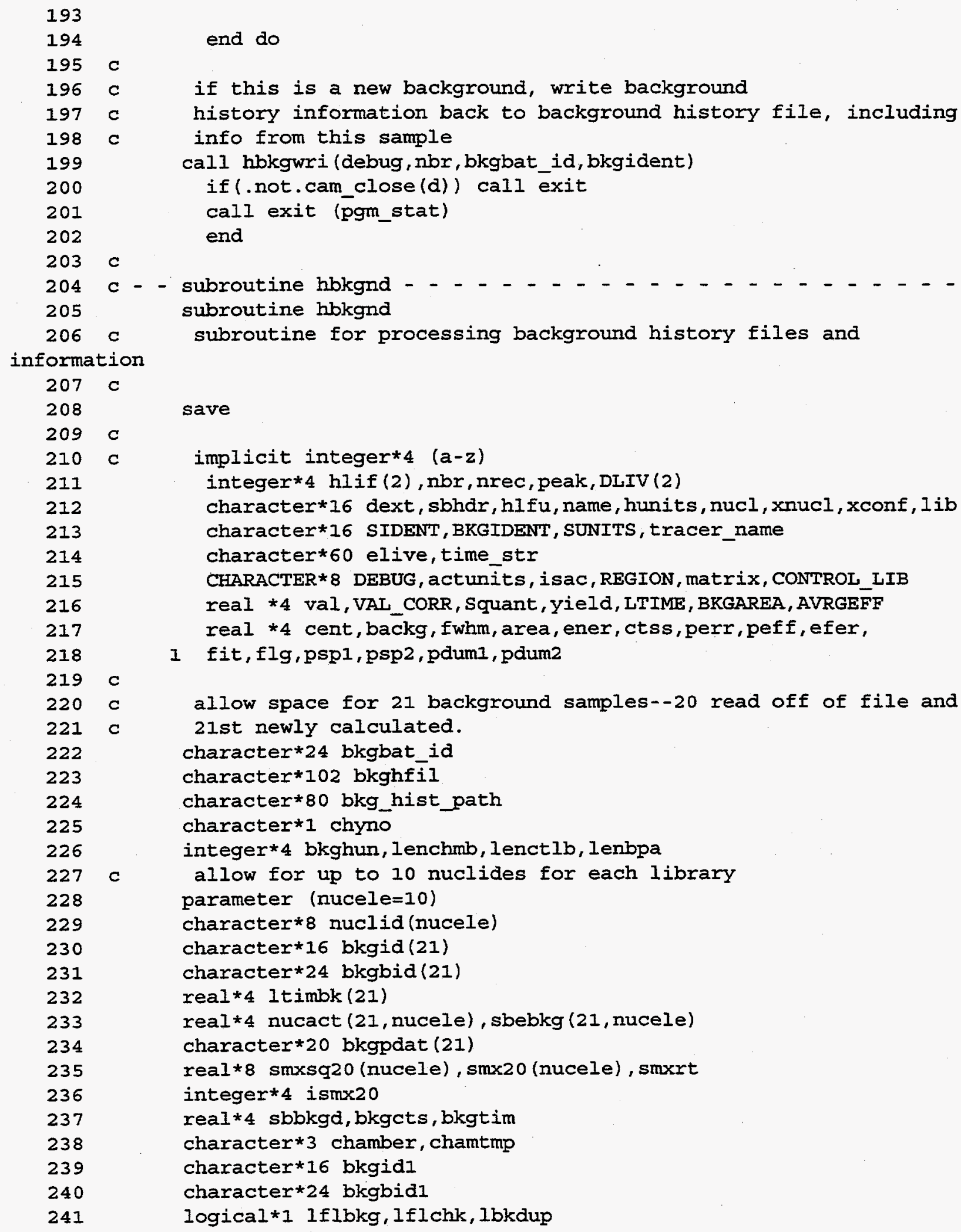




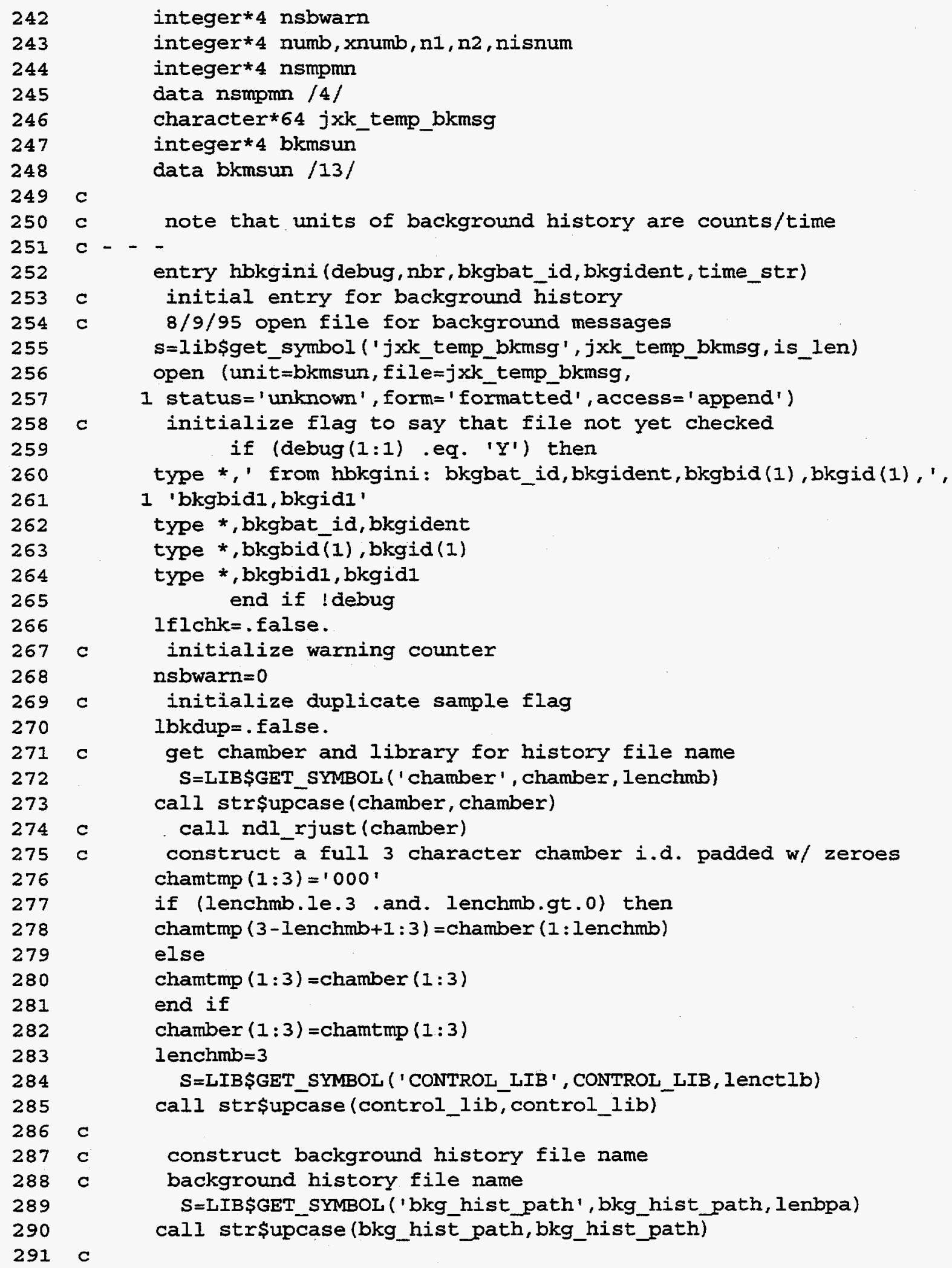


Appendix 2.D Listing of FORTRAN code BKGD_HIST.FOR, file version as of January 4, 1996

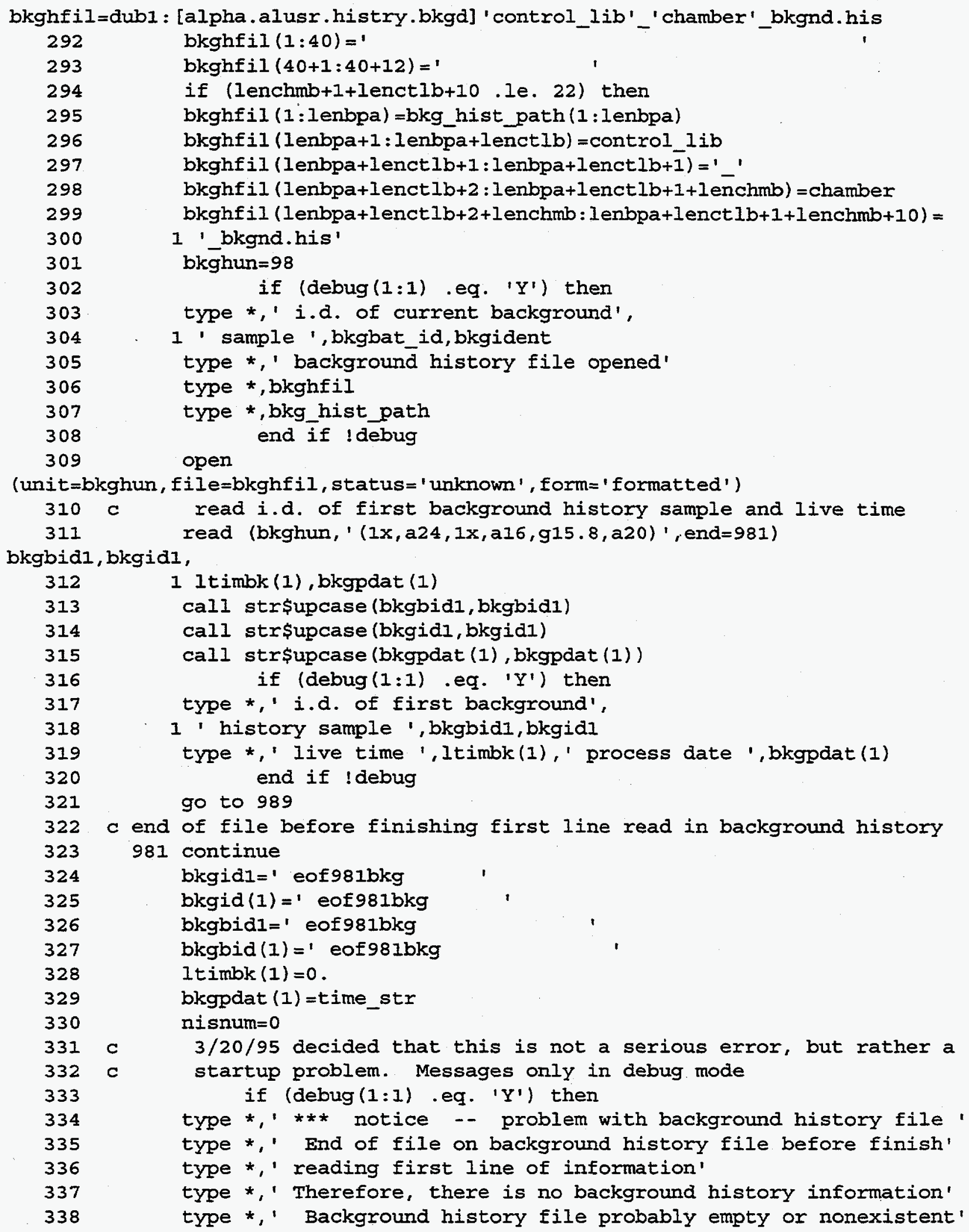


Appendix 2.D Listing of FORTRAN code BKGD HIST.FOR, file version as of January 4, 1996

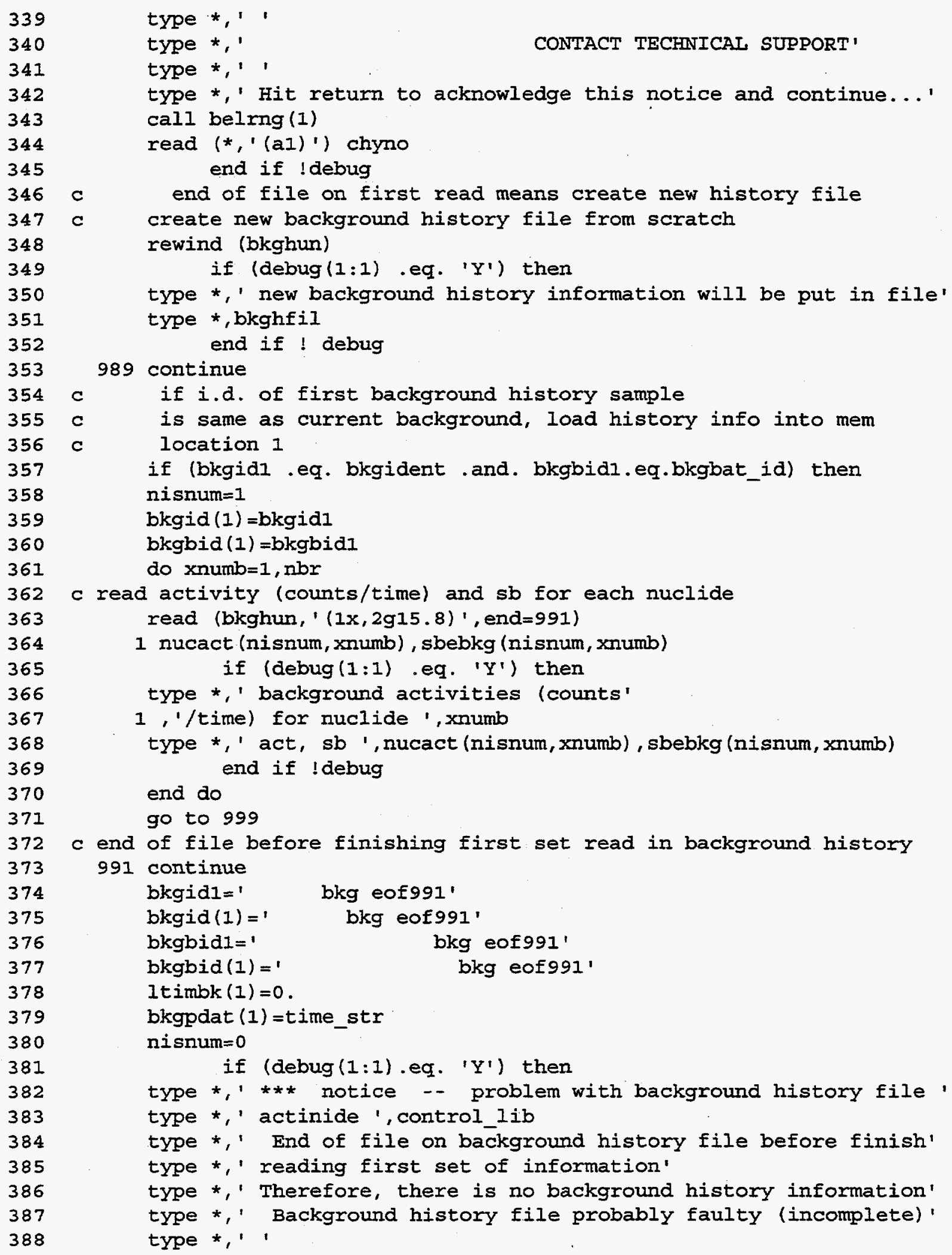

339

340

341

342

343

344

345

346

347

348

349

350

351

352

353

354

355

356

357

358

359

360

361

362

363

364

365

366

367

368

369

370

371

372

373

374

375

376

377

378

379

380

381

382

383

384

385

386

387

388

type *,' '

type *,'

type *,' '

CONTACT TECHNICAL SUPPORT'

type *,' Hit return to acknowledge this notice and continue...'

call beling (I)

read (*,' (aI)') chyno

end if !debug

c end of file on first read means create new history file

c create new background history file from scratch

rewind (bkghun)

if (debug $(1: 1)$.eq. ' $Y$ ') then

type *,' new background history information will be put in file'

type *,bkghfil

end if ! debug

989 continue

c if i.d. of first background history sample

c is same as current background, load history info into mem

c location 1

if (bkgidl .eq. bkgident . and. bkgbidl.eq.bkgbat_id) then

nisnum $=1$

bkgid (1) =bkgid1

bkgbid (1) =bkgbid 1

do xnumb=1, nbr

$c$ read activity (counts/time) and sb for each nuclide

read (bkghun, ' $(1 \mathrm{x}, 2 \mathrm{~g} 15.8$ ) ', end=991)

1 nucact (nisnum, xnumb), sbebkg (nisnum, xnumb)

if (debug $(1: 1)$.eq. 'Y') then

type *,' background activities (counts'

1 ,'/time) for nuclide ', xnumb

type *,' act, sb ', nucact (nisnum, xnumb), sbebkg (nisnum, xnumb) end if !debug

end do

go to 999

$c$ end of file before finishing first set read in background history

991 continue

bkgid1=' bkg eof991'

bkgid $(1)=$ ' bkg eof $991^{\prime}$

bkgbid1 = '

bkg eof 991 '

bkgbid (1) ='

bkg eof 991 '

$1 t i m b k(I)=0$.

bkgpdat (1)=time_str

nisnum $=0$

if (debug $(1: 1)$.eq. ' $Y^{\prime}$ ) then

type *,' *** notice -- problem with background history file '

type *,' actinide ', control_lib

type *,' End of file on background history file before finish'

type *,' reading first set of information'

type *," Therefore, there is no background history information'

type *,' Background history file probably faulty (incomplete)'

type *,' 
Appendix 2.D Listing of FORTRAN code BKGD HIST.FOR, file version as of January 4, 1996

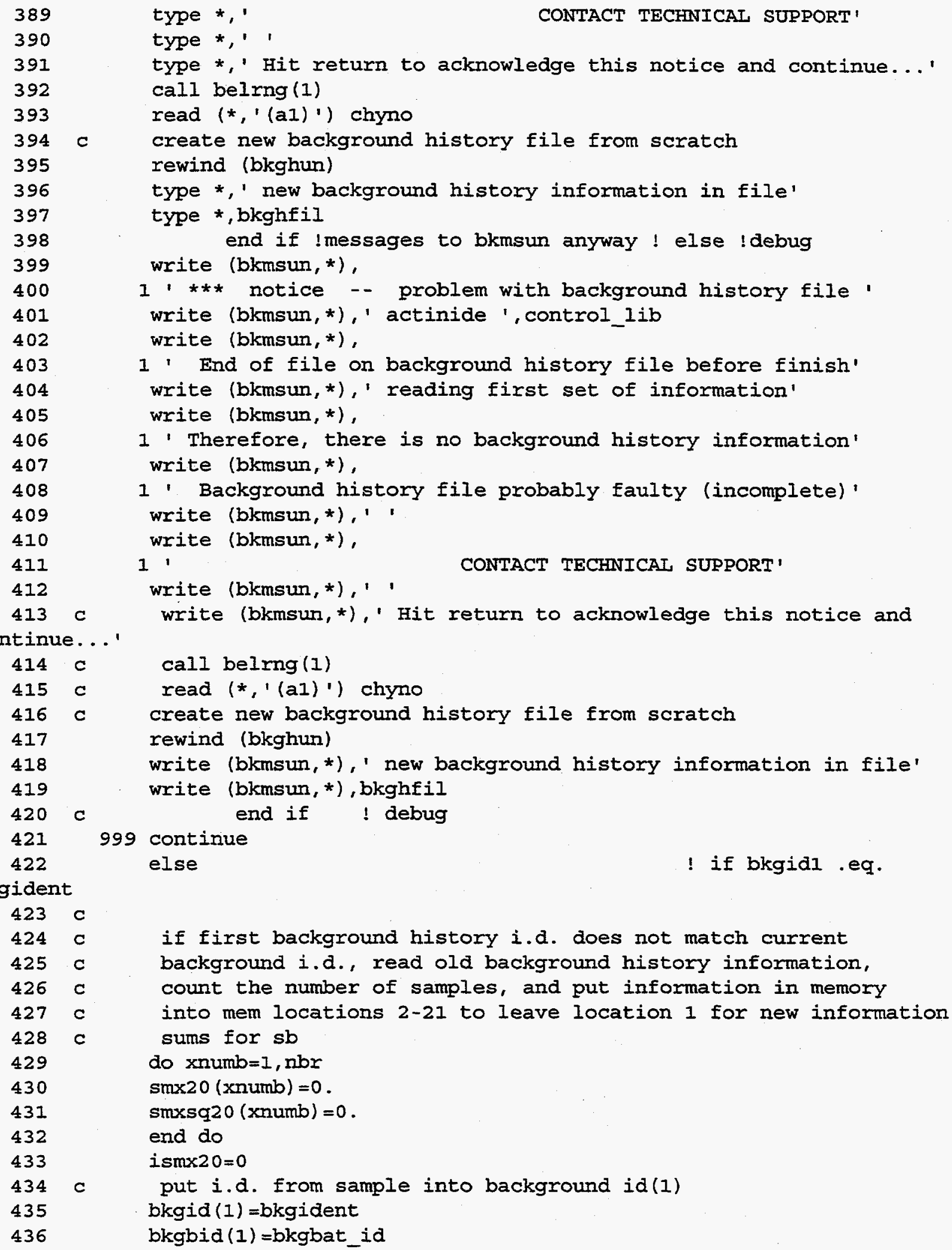


Appendix 2.D Listing of FORTRAN code BKGD HIST.FOR file version as of January 4, 1996

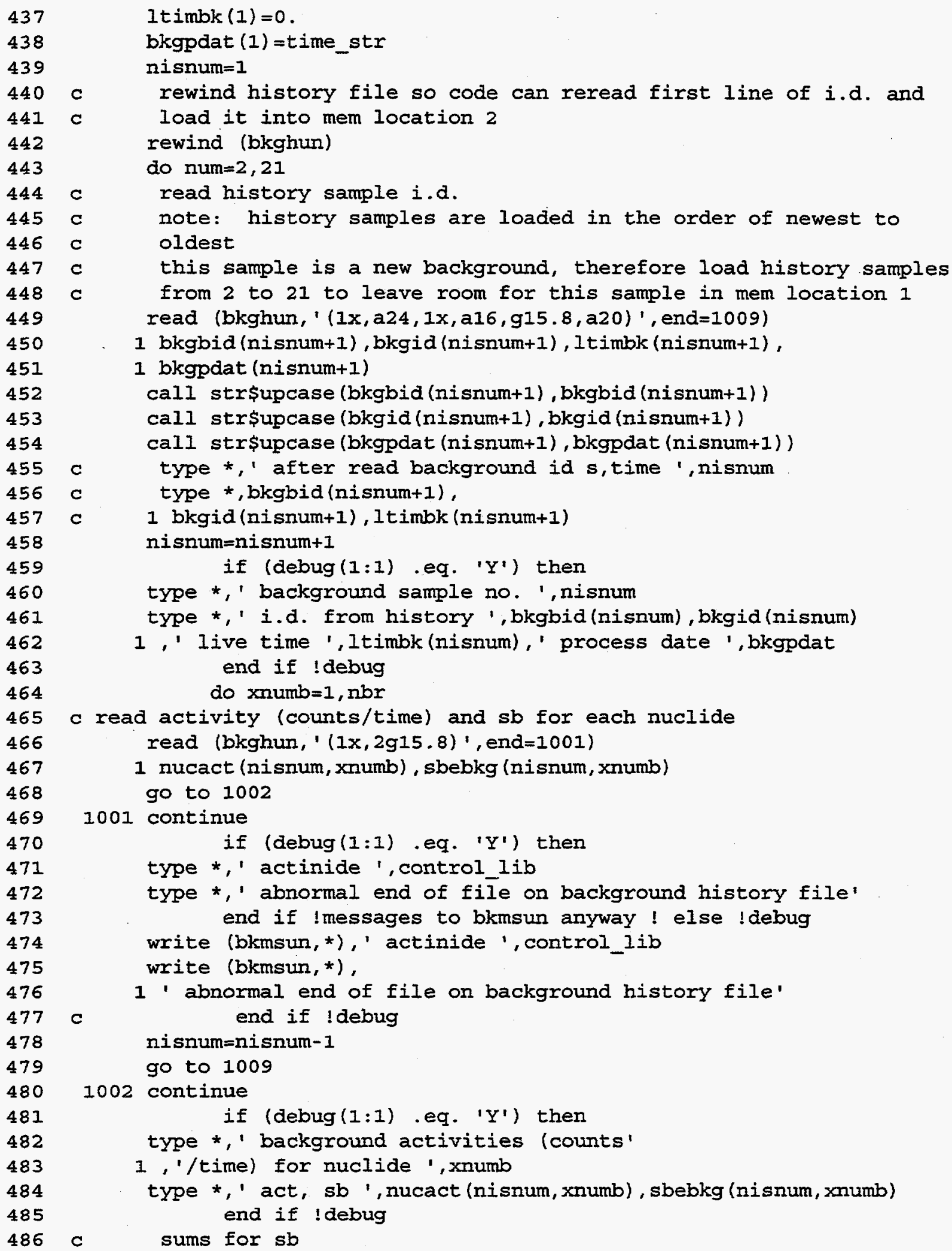


Appendix 2.D Listing of FORTRAN code BKGD HIST.FOR, file version as of January 4, 1996

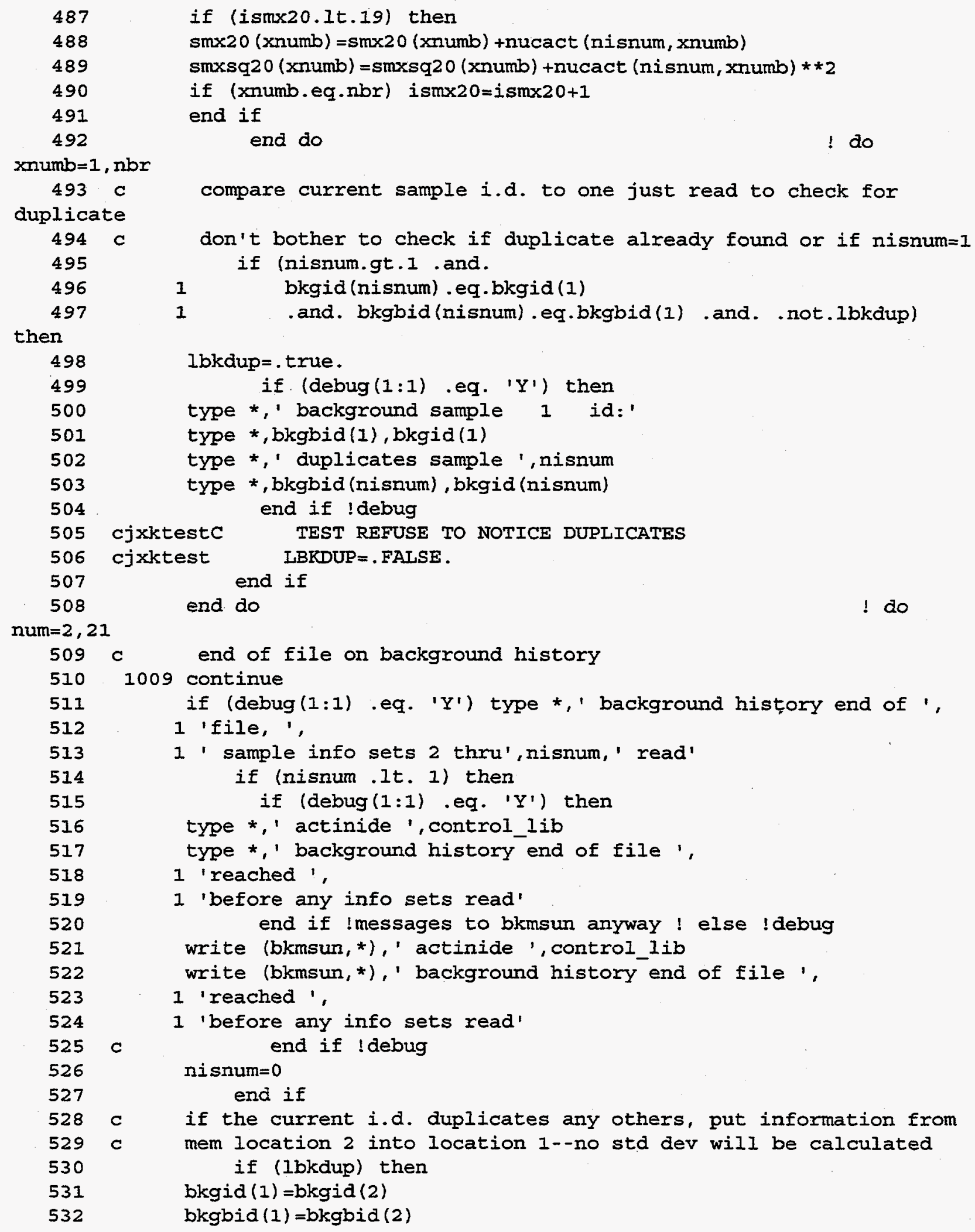

487

488

489

490

491

492

xnumb=1, nbr

$493 \mathrm{C}$

duplicate

$494 \mathrm{C}$

495

496

497

then

498

499

500

501

502

503

504

505

506

507

508

num $=2,21$

$509 \mathrm{C}$

510

511

512

513

514

515

516

517

518

519

520

521

522

523

524

525

526

527

528

$529 \mathrm{C}$

530

531

532

if (ismx20.1t.19) then

smx20 ( $x$ numb) $=s m \times 20$ ( $x$ numb) +nucact (nisnum, $x$ numb)

smxsq20 (xnumb) $=\operatorname{smxsq20}(x n u m b)+$ nucact (nisnum, xnumb) $* * 2$

if (xnumb.eq.nbr) ismx $20=i \operatorname{smx} 20+1$

end if

end do

! do

compare current sample i.d. to one just read to check for

don't bother to check if duplicate already found or if nisnum=I if (nisnum.gt.I and.

1 bkgid(nisnum) .eq.bkgid(1)

1 .and. bkgbid (nisnum) .eq.bkgbid(1) .and. .not.lbkdup) 
Appendix 2.D Listing of FORTRAN code BKGD HIST.FOR, file version as of January 4, 1996

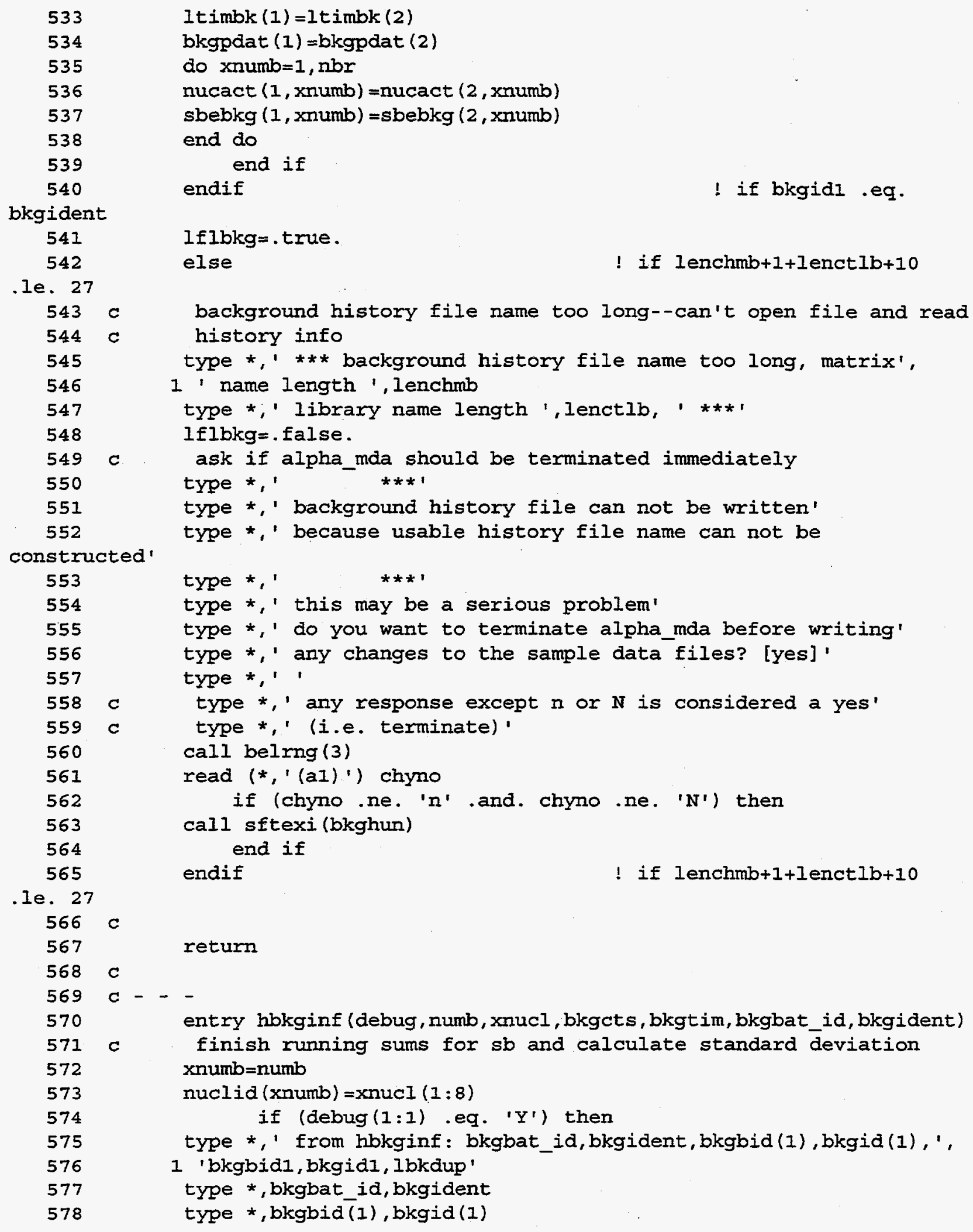


Appendix 2.D Listing of FORTRAN code BKGD HIST.FOR, file version as of January 4, 1996

579

580

$581 \mathrm{C}$

$582 \mathrm{C}$

583

584

585

1

586

587

588

589

590

$591 \mathrm{C}$

592

593

594

595

596

597

598

nsmpmn

599

600

601

602

603

604

605

606

607

608

609

610

$611 \mathrm{C}$

probably due

$612 \mathrm{c}$

613

614

615

616

617

618

619

620

621

622

623

624

625 type *, bkgbidl, bkgid1, 1bkdup end if !debug

if background $i . d$. does not match first line read from history file and no other duplicates, finish running sums for sb

if ( (bkgidl.ne.bkgident .or. bkgbidl.ne.bkgbat_id)

1 . and. not.lbkdup) then

if (debug(I:I) .eq. ' $Y^{\prime}$ ) type *,' background sample (=record 1 ).

1 ' nuclide ', xnumb,' counts/sec ',bkgcts,' time ',bkgtim

nucact $(1$, xnumb) $=$ bkgcts

1timbk $(1)=$ bkgtim

$\operatorname{smx} 20(x n u m b)=\operatorname{smx} 20(x n u m b)+$ nucact $(1, x n u m b)$

smxsq20 (xnumb) =smxsq20 (xnumb) +nucact $(1, x n u m b) * * 2$

increment counter for sum of 20 . do this on first

nuclide

if (xnumb .eq. 1) then

ism $\times 20=i \sin \times 20+1$

end if

calculate std dev

if (ismx20.gt.1) then

1/12/95 decision made that standard deviation for fewer than

(=4) samples not worthwhile, so make special provisions

if (ismx20.ge.nsmpmn) then

$\operatorname{smxrt}=(\operatorname{sinxsq20}(x \operatorname{mumb})-\operatorname{smx} 20(x n u m b) * * 2 /$

$1 \operatorname{dfloat}($ ism $\times 20)$ )/dfioat (ism $\times 20-1$ )

if (debug $(1: 1$ ). eq. ' $Y$ ') then

type *,' terms for backgr std dev for ', 'nuclide ', xnumb

type *,' smxsq20, smx20, ismx20, sum term'

type *, smxsq20 (xnumb), smx20 (xnumb), ismx20, smxrt end if ! debug

c check to see that std deviation will be real

if (smxrt.1t. 0.) then

$\operatorname{sinxrt}=0$.

if sum of terms for root is small, then negative value is

to truncation error

if (dabs (smxrt).gt. 1.d-8*nucact $(1, x n u m b) * * 2$ ) then

if (debug $(1: 1)$.eq. ' $Y$ ') then

type *, '******1

type *,' actinide ', control_lib

type *,' backg standard deviation not real for nuclide ', xnumb

type *,' zero will be returned'

type *,' this may be a serious problem'

type *,' '

type *,' Hit return to acknowledge this notice and continue...'

call belmg (1)

read (*,' (a1)') chyno

end if !messages to bkmsun anyway ! else ! debug

write (bkmsun, *), '****** 
Appendix 2.D Listing of FORTRAN code BKGD HIST.FOR. file version as of January 4, 1996

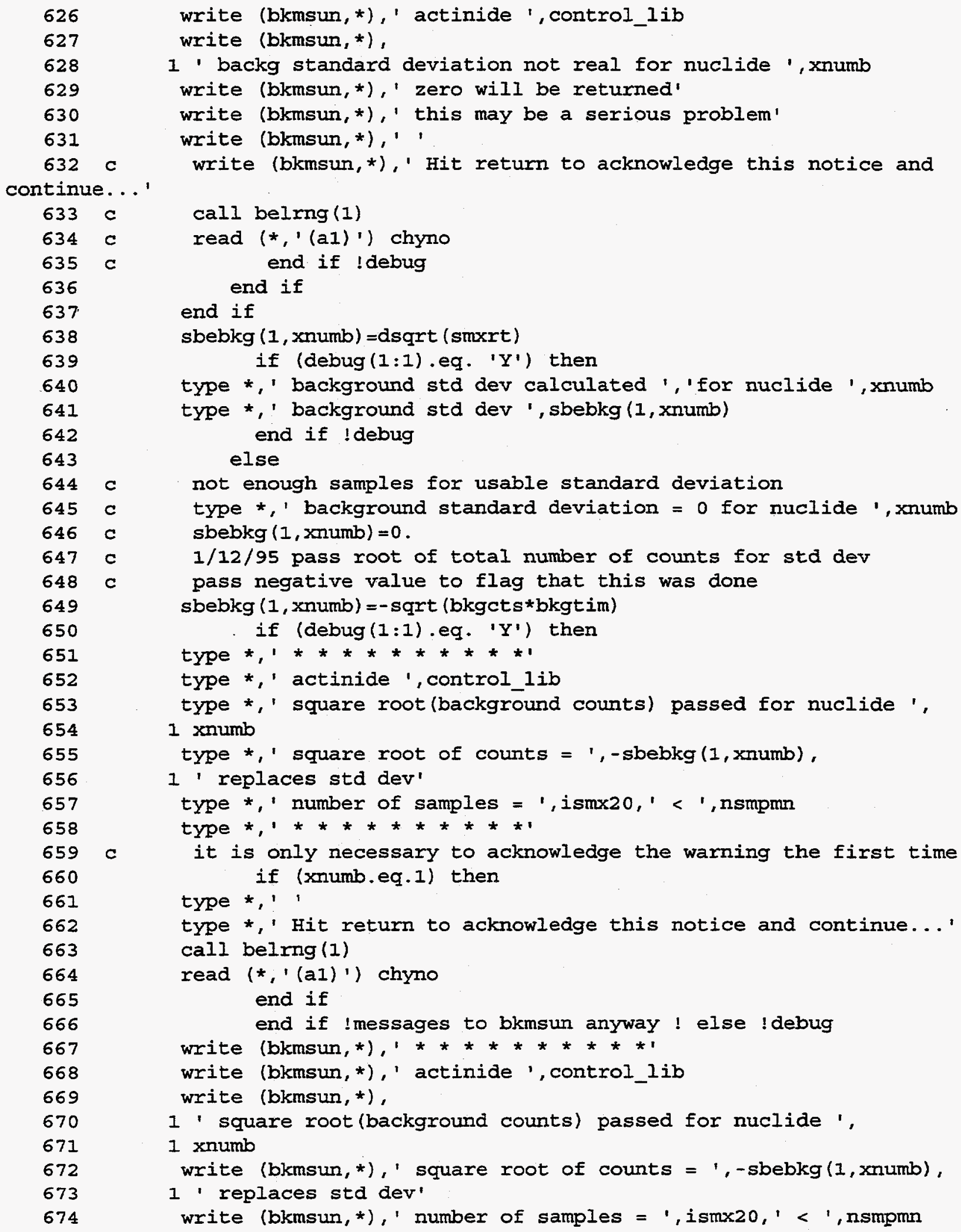

626

627

628

629

630

631

$632 \mathrm{C}$ continue...'

$633 \mathrm{c}$

$634 c$

$635 \mathrm{C}$

636

637

638

639

640

641

642

643

644 C

$645 \mathrm{C}$

$646 \mathrm{C}$

647 C

648 C

649

650

651

652

653

654

655

656

657

658

659

660

661

662

663

664

665

666

667

668

669

670

671

672

673

674 
Appendix 2.D Listing of FORTRAN code BKGD_HIST.FOR, file version as of January 4.1996

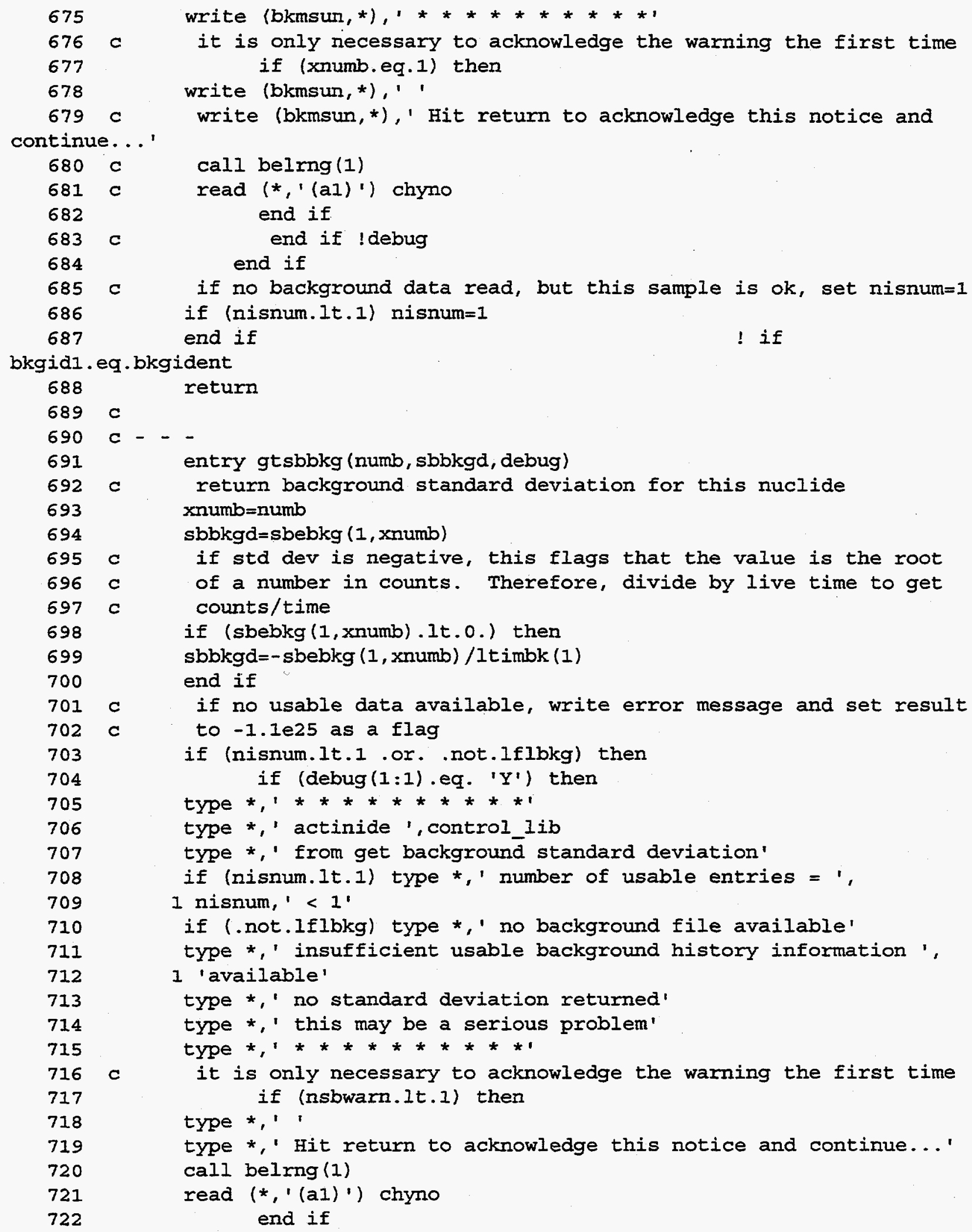


Appendix 2.D Listing of FORTRAN code BKGD_HIST.FOR, file version as of January 4.1996

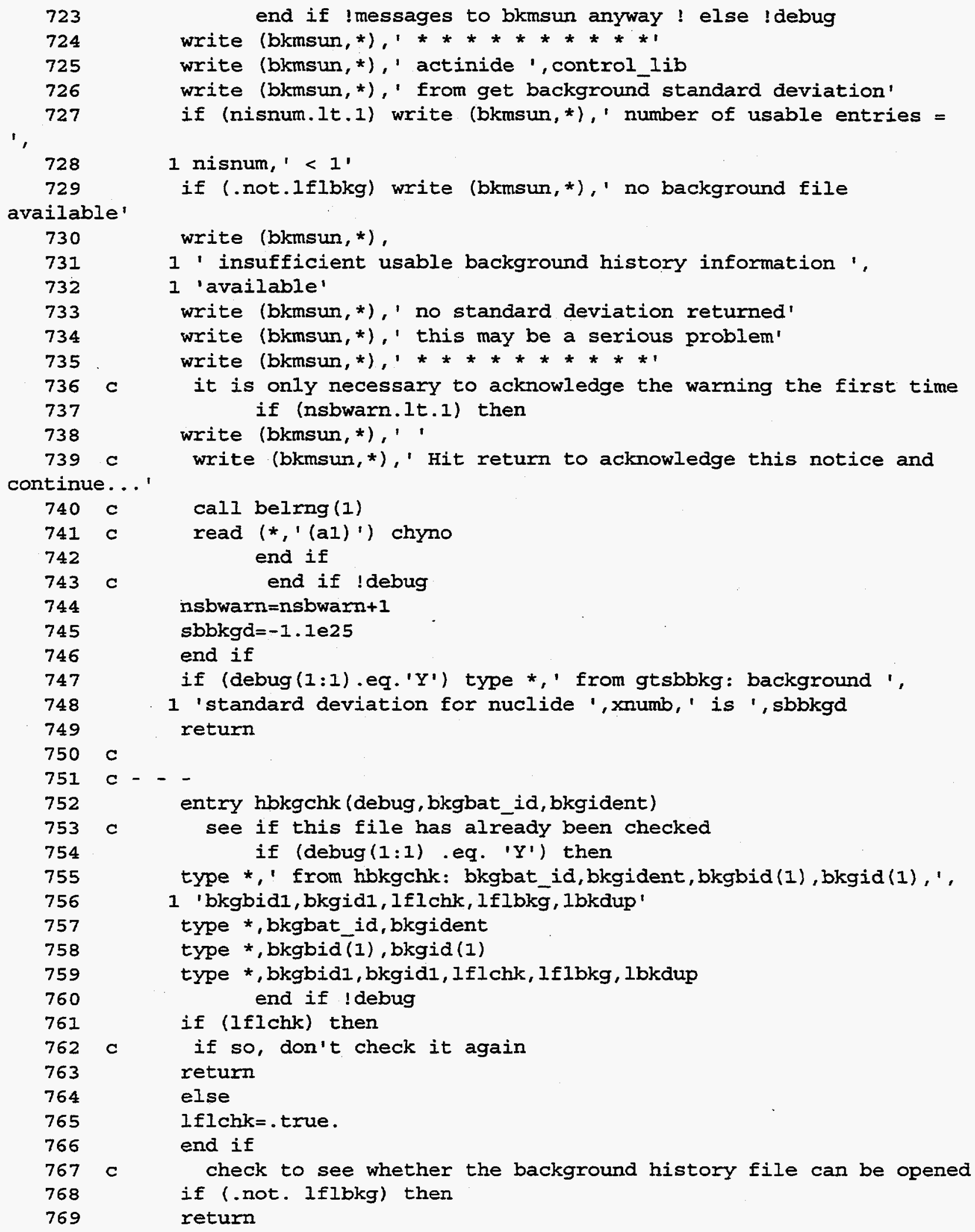

723

724

725

726

727

',

728

729

available'

730

731

732

733

734

735

$736 \mathrm{C}$

737

738

$739 \mathrm{C}$ continue...'

$740 \mathrm{C}$

741 C

742

743

744

745

746

747

748

749

750

751

752

753

754

755

756

757

758

759

760

761

762

763

764

765

766

767

768

769

end if !messages to bkmsun anyway ! else !debug

write (bkmsun, *), $1 * * * * * \star \star * * * * 1$

write (bkmsun, *),' actinide ', control_lib

write (bkmsun,*), ' from get background standard deviation'

if (nisnum.lt.1) write (bkmsun,*),' number of usable entries =

1 nisnum, ' $<1$ '

if (.not.lflbkg) write (bkmsun,*),' no background file

write (bkmsun, *),

1 ' insufficient usable background history information ',

1 'available'

write (bkmsun,*), ' no standard deviation returned'

write (bkmsun,*), ' this may be a serious problem'

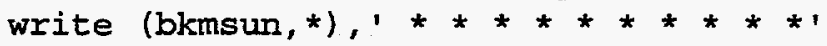

it is only necessary to acknowledge the warning the first time if (nsbwarn.1t.1) then

write (bkmsun, *),' '

write (bkmsun,*),' Hit return to acknowledge this notice and

call belrng (1)

read (*,' (aI)') chyno

end if

end if !debug

nsbwarn=nsbwarn+1

sbbkgd $=-1.1 \mathrm{e} 25$

end if

if (debug(I:I).eq.'Y') type *,' from gtsbbkg: background ',

1 'standard deviation for nuclide ', xnumb,' is ', sbbkgd return

C

$c$ - -

C

entry hbkgchk (debug, bkgbat_id, bkgident)

see if this file has already been checked

if (debug(1:1) eq. ' $Y$ ') then

type *,' from hbkgchk: bkgbat_id,bkgident,bkgbid(1), bkgid(1), ',

1 'bkgbidl, bkgid1, lflchk, lflbkg, 1bkdup'

type *, bkgbat_id,bkgident

type *,bkgbid(1), bkgid (1)

type *, bkgbidl, bkgid1, Iflchk, Iflbkg, lbkdup end if ! debug

if ( $I f l c h k$ ) then

c if so, don't check it again

return

else

Iflchk = true.

end if

c check to see whether the background history file can be opened if (.not. Iflbkg) then

return 
Appendix 2.D Listing of FORTRAN code BKGD HIST.FOR, file version as of January 4, 1996

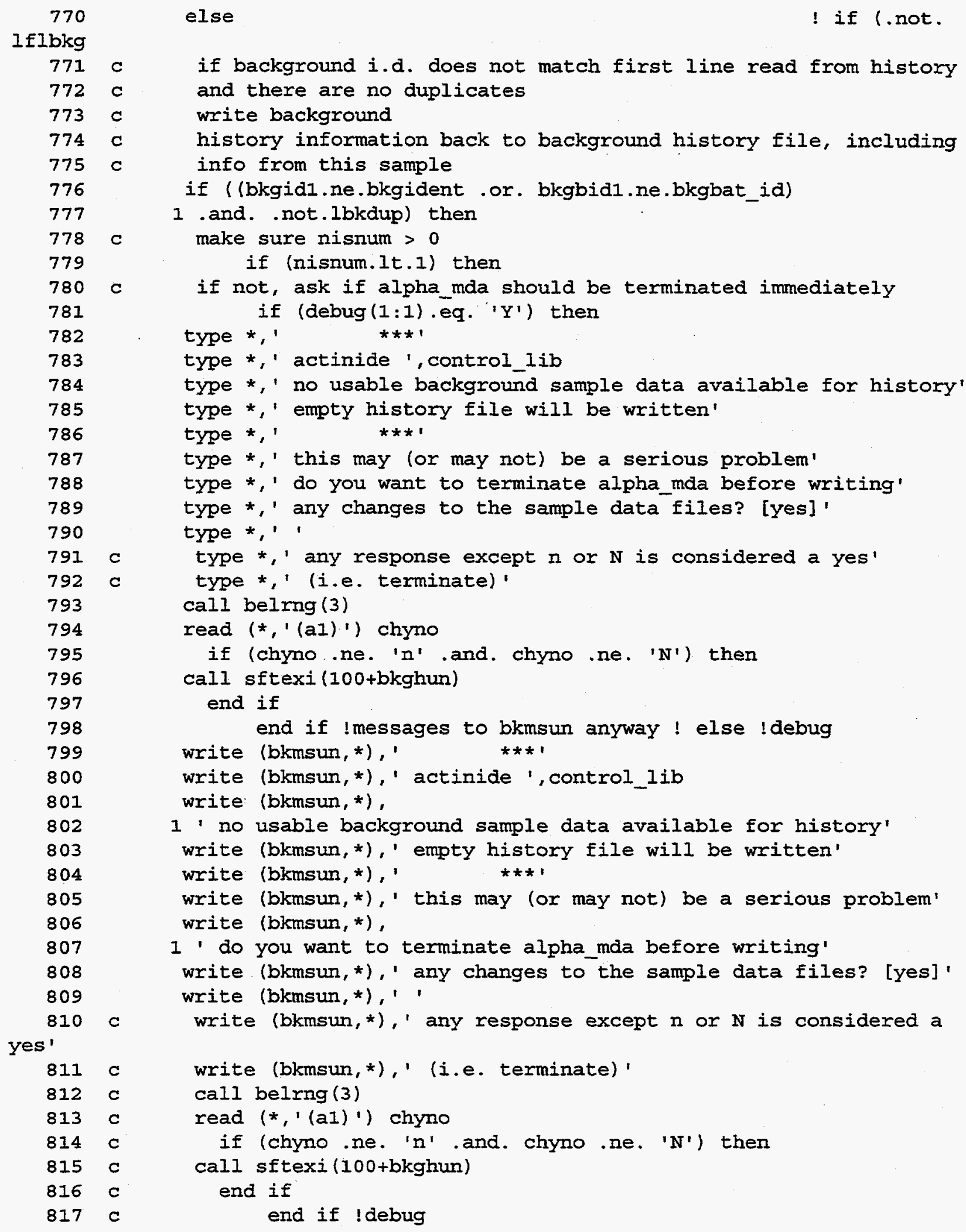


Appendix 2.D Listing of FORTRAN code BKGD HIST.FOR, file version as of January 4, 1996

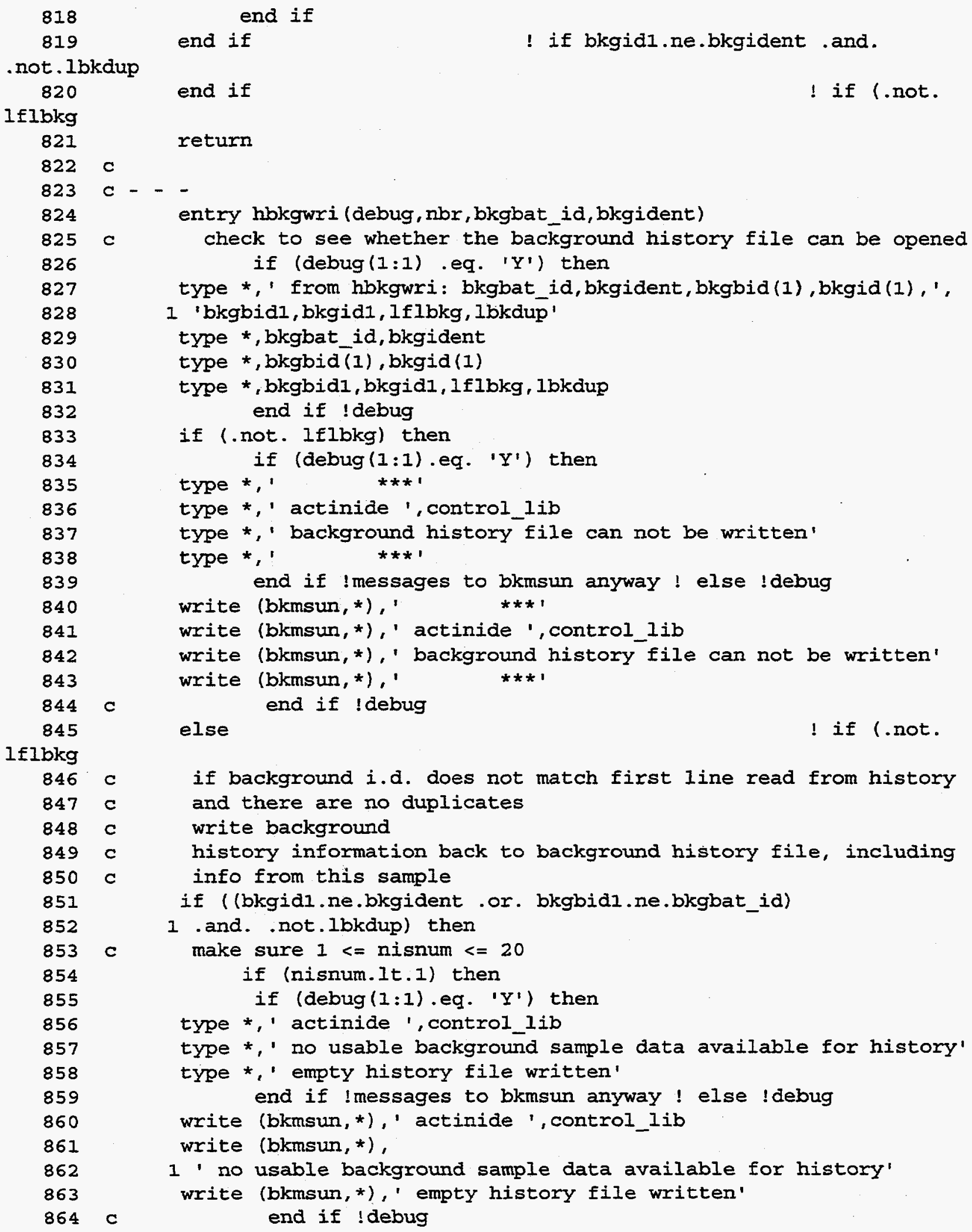


Appendix 2.D Listing of FORTRAN code BKGD_HIST.FOR, file version as of January 4,1996

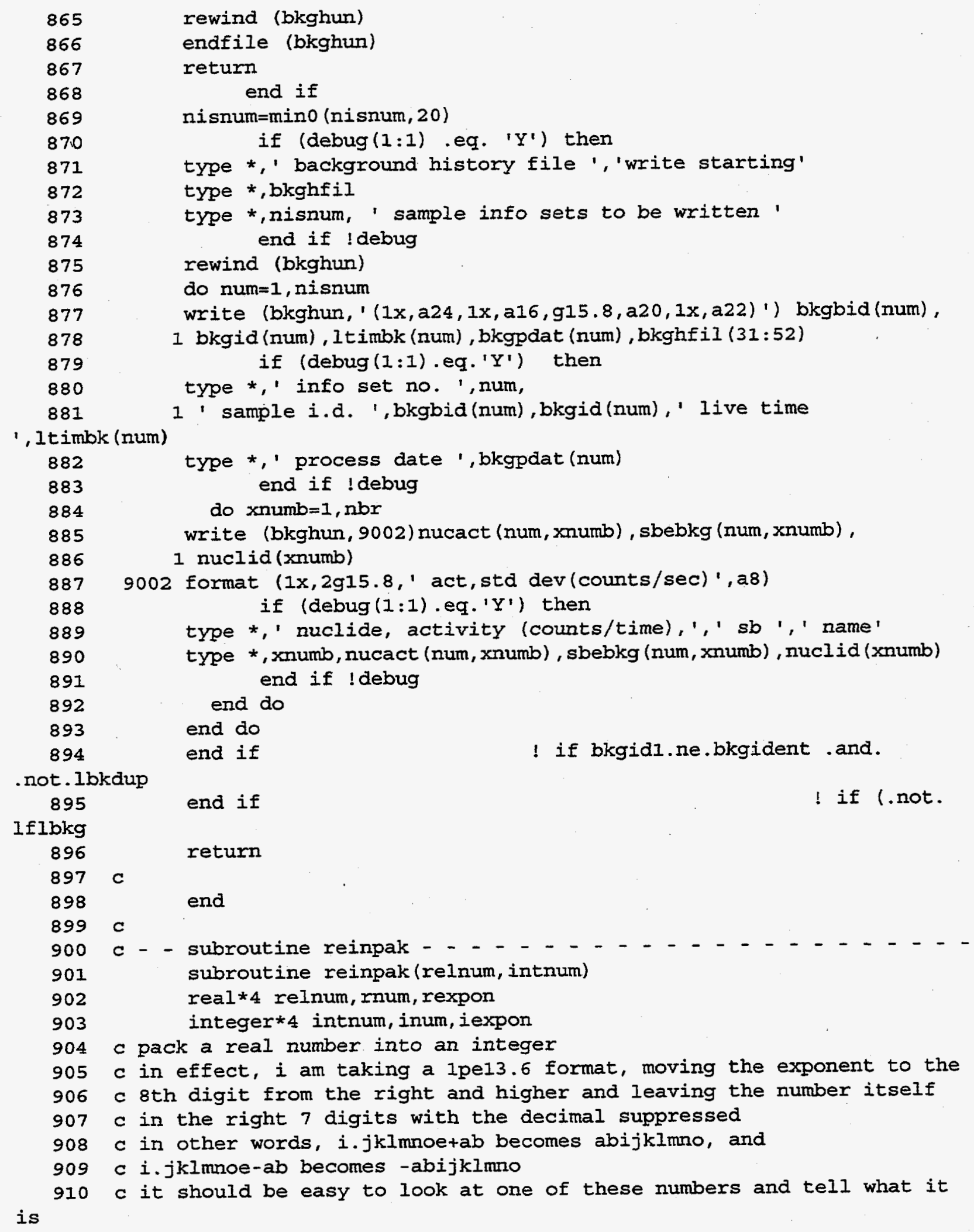


Appendix 2.D Listing of FORTRAN code BKGD HIST.FOR, file version as of January 4. 1996

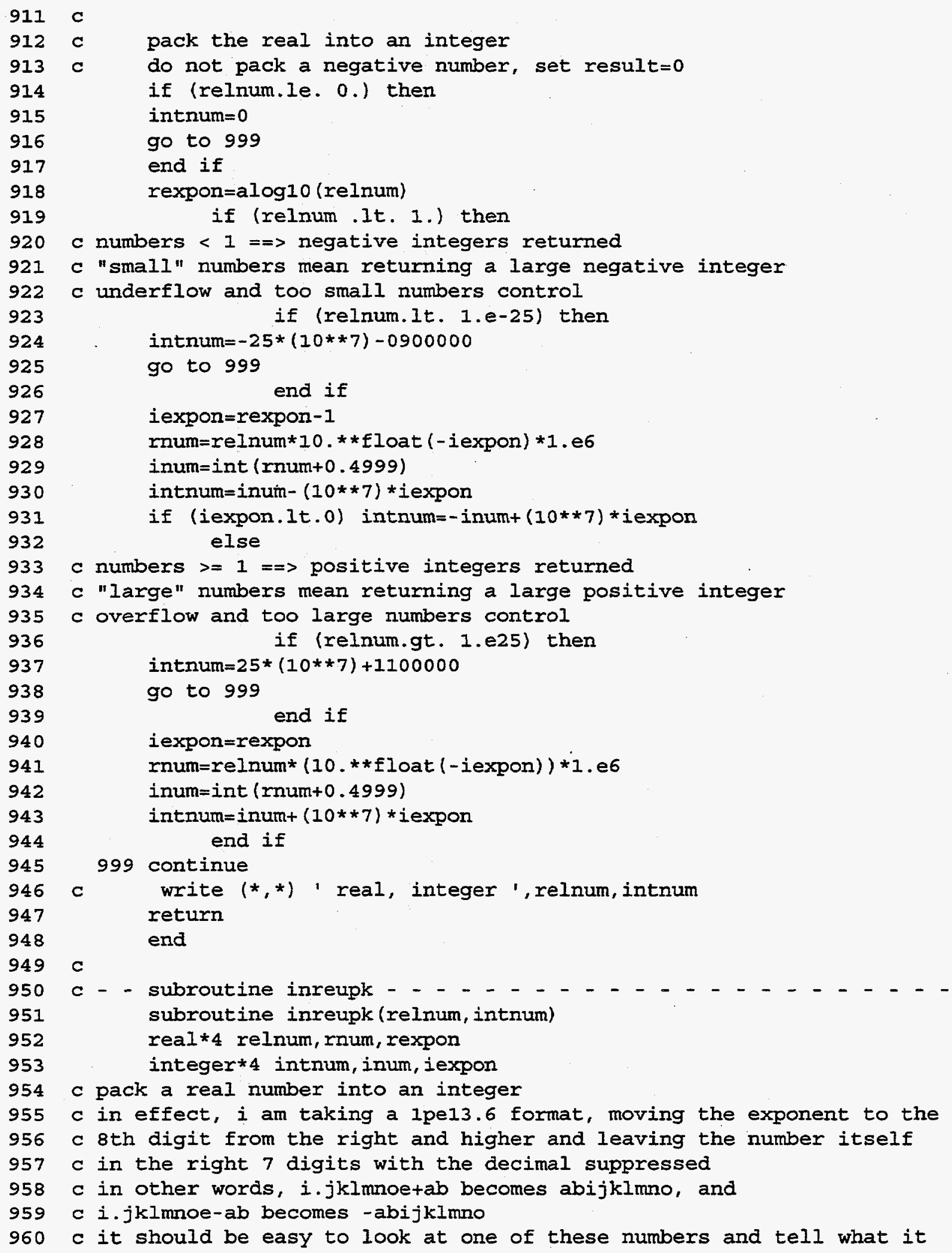


is

$961 C$

$962 \mathrm{C}$

963 C

964

965

966

967

968

969

970

971

972

973

974

975

976

977

978

979

980

981

982

983

984

985

986

987

988

989

990

991

992

993

994

995

996

997

998

999

1000

1001

1002

1003

1004

1005

1006

1007

1008

1009

c unpack the integer and reconstruct the real

inputting a zero means returning a zero

if (intnum.eq.0) then

relnum $=0$.

go to 999

end if

iexpon=intnum/ $(10 * * 7)$

if (intnum.1t.0) then

c numbers $<1==>$ negative integers returned

inum $=-$ intnum $+(10 * 7)$ *iexpon

rnum $=$ float (inum) $/ 1$.e6

rexpon=float (iexpon)

relnum $=$ rnum $* 10 . *$ rexpon

else

c numbers $>=1==>$ positive integers returned

inum $=$ intnum $-(10 * * 7) *$ iexpon

rnum $=$ float (inum) $/ 1 . e 6$

rexpon $=$ float $($ iexpon)

relnum $=$ rnum $* 10 . * *$ rexpon

end if

999 continue

c write $(*, *)$ ' real, integer ', relnum, intnum return

end

c - - subroutine sftexi - - - - - - - - - - - - - - - -

subroutine sftexi(iflexi)

integer*4 iflexi

character*1 chyno

c soft exit subroutine

c ask if the process.com should exit

type *,'

*** '

type *,' alpha_mda is exiting at your request'

type *,' do you want process.com to terminate also?'

type *,' if not,'

type *,' process.com will continue processing samples'

type *, ',

type *,' any response except $n$ or $N$ is considered a yes'

type *,' (i.e. terminate)'

call belrng (3)

read $(*, '(a 1)$ ') chyno

if (chyno .eq. ' $N$ ') chyno='n'

$c$ write messages to tty and get flag symbol (alphaerr) ready to send

c back to process.com

if (chyno.ne. ' $n$ ') then

type *,' you told process.com to quit'

else

type *,' you told process.com to continue' 
Appendix 2.D Listing of FORTRAN code BKGD_HIST.FOR, file version as of January 4, 1996

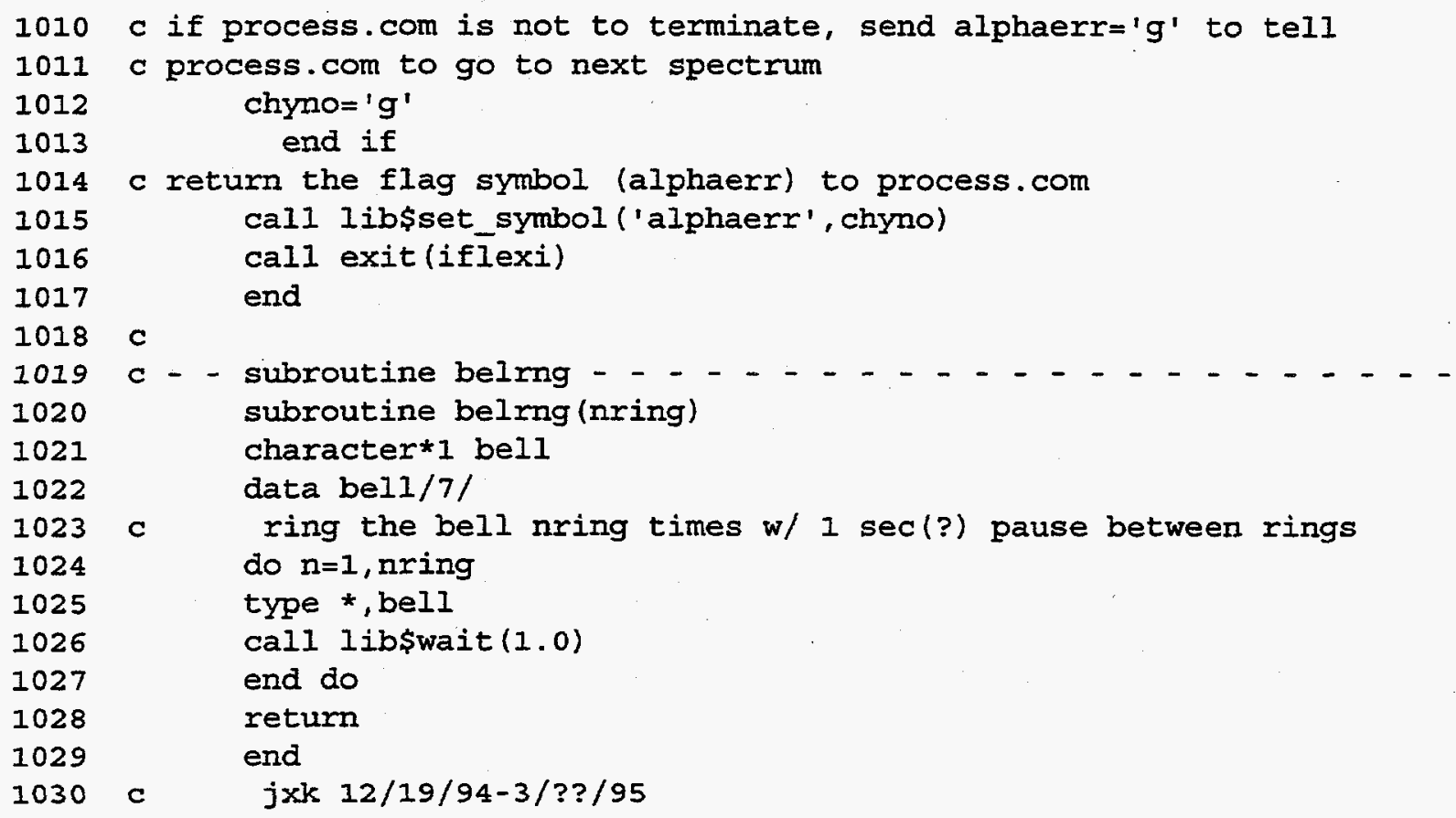




\section{APPENDIX 2.E LISTING OF DCL FILE NDAL_BKGD_HISTRY.COM, FILE VERSION AS OF AUGUST 16,1995}

File is located on system B disk at dubo:[000000.nd_root.ndamscust]

Thu Aug 24 13:04:57 EDT 1995

$\$$ cat $-\mathrm{n}$ blkghist 2 .com

$1 \$$ ! set ver

$2 \$$ ! jxk 4/95-5/95 process background file to make sure it goes into

$3 \$ !$ history

$4 \$$ if $\mathbf{f}$ \$edit (f\$extract $(0,1, y 12$ bioassay), "upcase") .eqs. "Y" -

"Y"

5 .or. f\$edit (f\$extract $(0,1$, new_reagent_calc), "upcase") .eqs.

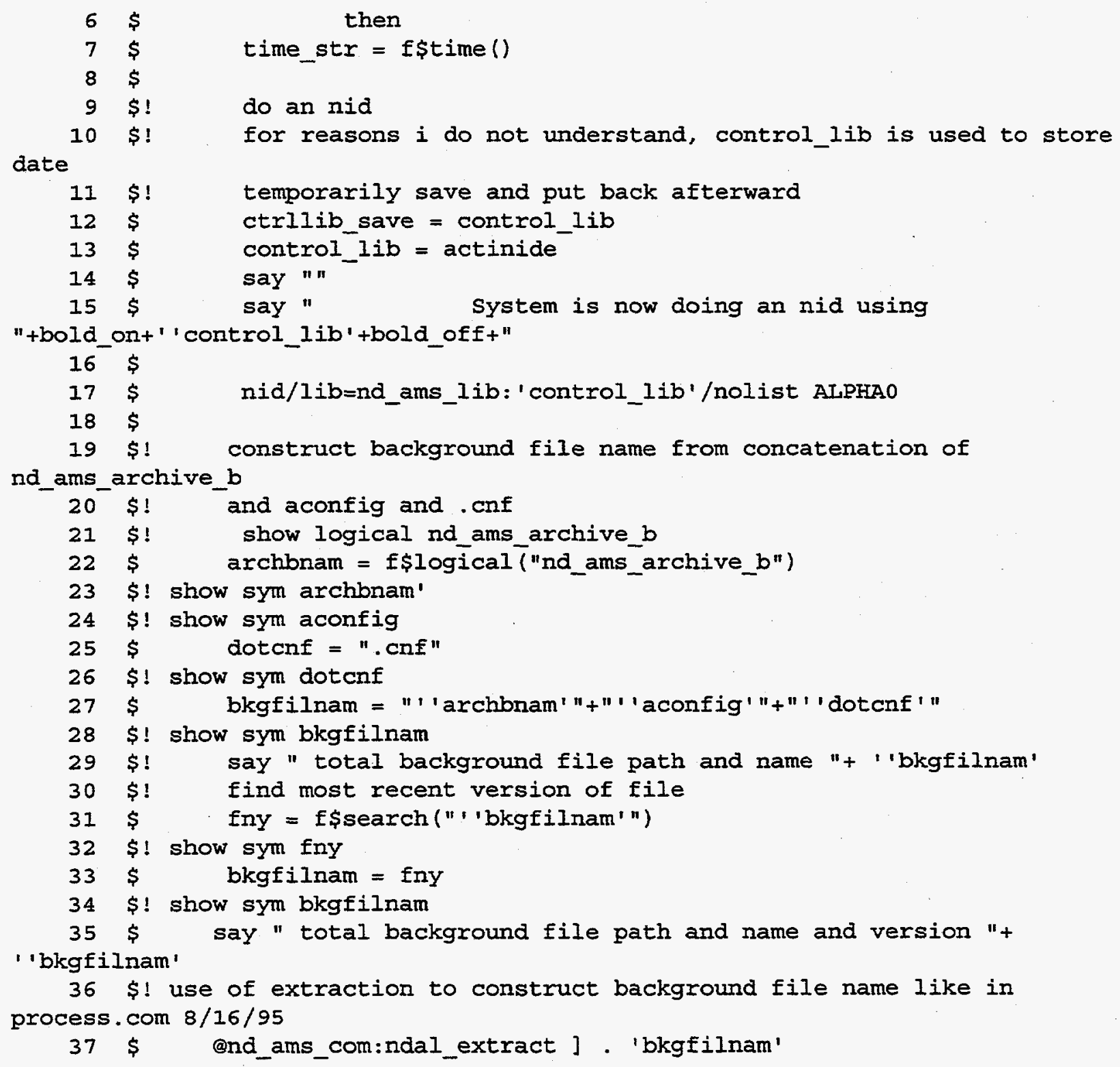




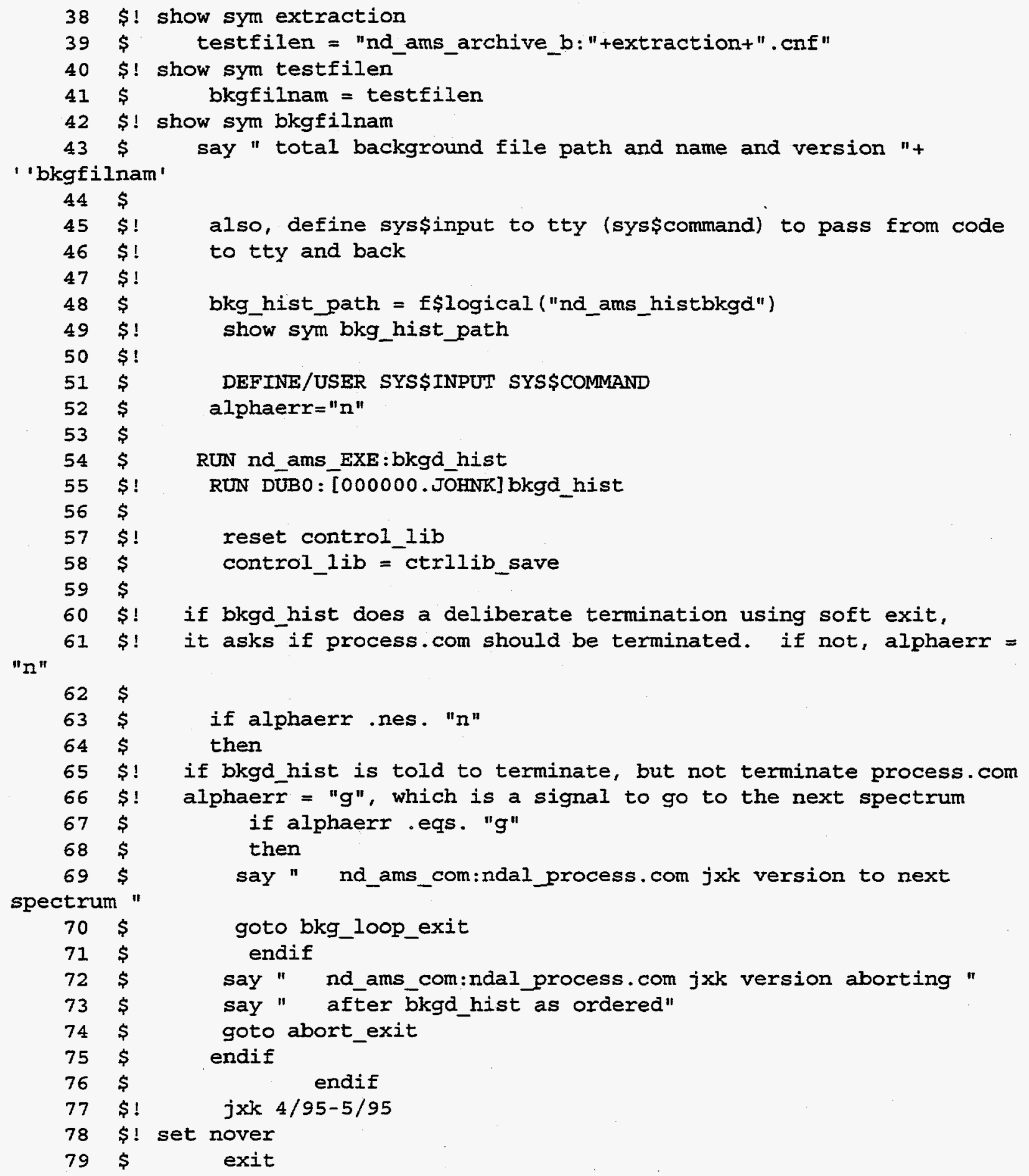




\title{
APPENDIX 2.F LISTING OF FORTRAN CODE FORTOPEN.FOR, FILE VERSION AS OF JANUARY 4, 1996
}

\author{
File is located on system B disk at dubo:[000000.nd_root.ndamscust.for] \\ Mon Jan 8 11:25:09 EST 1996 \\ $\$$ cat $-n$ fortopen. for \\ 1 c this is a small fortran code whose mission is to open the \\ 2 c file file_fortopen formatted with fortran file attributes. \\ 3 c the intent is that one can write from both vms and fortran \\ $4 \mathrm{c}$ and have a file which will print correctly. \\ 5 c the reason for this file's existence is that opening a file \\ 6 c with the open command from vms creates a file with different \\ 7 c record attributes from those from a fortran open. \\ $8 \mathrm{c}$ the result of the differences is that the lines from fortran \\ 9 c do not have the "proper" carriage return-line feed sequence. \\ $10 \mathrm{c}$ as a consequence, trying to print a file which has been opened \\ $11 \mathrm{c}$ with vms and subsequently written in append mode with fortran \\ 12 c creates very long lines without cr-lf to separate them. \\ 13 c j.r. Kirkpatrick, lockheed martin (oak ridge national lab) \\ 14 C (423) 574-8676 9/8/95 \\ 15 character $* 80$ file_fortopen \\ 16 \\ $s=1$ ib\$get_symbol ('file_fortopen', file_fortopen, length) \\ open (unit=11, file=file_fortopen, \\ 1 form = ' formatted' ) \\ close (unit=11, disp=' keep') \\ call exit \\ end
}





\section{APPENDIX 2.G INSTRUCTIONS FOR DEALING WITH REAGENT BLANK SAMPLE EXCEEDS ALARM LIMIT CONDITION}

A blank sample exceeding the alarm limit is expected to be a rare condition. When the ALPHA_MDA program first begins to process a reagent blank sample, it asks the operator for a state of the sample. Possible states are "1) SAMPLE SPILLED," "2) CLOGGED RESIN," "3) CHARRING," "4) ACCIDENTAL SALVAGING," "5) REPROCESS," "6) RECOUNT," "7) OTHER," and "8) SAMPLE IS OK." The ALPHA_MDA program will check for exceeds alarm limit only if the operator has responded with "8) SAMPLE IS OK." One of the more likely causes of the exceeds alarm limit condition is that a regular sample is mistakenly labeled a reagent blank. That error would cause the sample to exceed the alarm limit because the alarm limit for reagent blanks is supposed to be lower than that for regular samples.

The ALPHA_MDA.FOR program allows the operator to make graceful exits when this condition is encountered. Five possible branches and sets of action are explained below. There may be other possible explanations for the condition or reasons for taking these branches. However, the steps to be taken represent the only branches that the author knows.

a. There is nothing wrong with the sample--the alarm limit is too low.

If this is really true, you should stop processing the sample and fix the alarm limit. The reasons for stopping immediately are as follows: (a) ALPHA_MDA.FOR will not put a blank sample that exceeds the alarm limit into the blank history file. Thus, this sample will not show up in its proper place in the blank history file, (b) Y12.FOR will flag the sample "EXCEEDS" in the "ddmmmyyyy.RES" flat file.

To stop processing, hit "RETURN" at the "do you want to terminate alpha_mda right now?" message. Then hit "RETURN" again at the "do you want process.com to terminate also?" message.

After you have fixed the alarm limit, edit NAMES.DAT so that you will start at this sample when you resume processing.

b. Lknow what is wrong with the sample and need to fix it before I go on.

If this is true, you should stop processing the sample and fix it.

To stop processing, hit "RETURN" at the "do you want to terminate alpha_mda right now?" message. Then hit "RETURN" again at the "do you want process.com to terminate also? message.

After you have fixed the sample, edit NAMES.DAT so that you will start at this sample (or skip it if that is what you decide to do) when you resume processing.

c. Iknow what is wrong with the sample. but I want to skip it and continue processing other samples.

Taking this branch implies that it does not matter if this sample does not go into the reagent blank history file because the sample is faulty, mislabelled, or something else. In this case, you will terminate ALPHA_MDA.FOR, but let PROCESS.COM continue.

To stop ALPHA_MDA but continue processing other samples, hit "RETURN" at the "do you want to terminate alpha_mda right now?" message. Then hit " $n$ " or " $N$ " at the "do you want 
process.com to terminate also?" message. PROCESS.COM will go to the "are you ready go to the next spectrum ?? (y/n) \#" message.

After you have fixed the sample, edit NAMES.DAT so that you will start at this sample (or skip it if that is what you decide to do) when you resume processing.

\section{d. Lknow what is wrong with the sample, but I want to process it. anyway.}

Because this sample exceeds the alarm limit, it will not go into the reagent blank history file. It will show up in the ddmmmyyyy.RES flat file with an "EXCEEDS" state. However, Y12.FOR will give you a chance to change this state to one of the error condition states, which are "1) SAMPLE SPILLED," "2) CLOGGED RESIN," "3) CHARRING," "4) ACCIDENTAL SALVAGING," "5) REPROCESS," "6) RECOUNT," and "7) OTHER."

To continue processing this sample, hit " $\mathrm{n}$ " or " $\mathrm{N}$ " at the "do you want to terminate alpha_mda right now?" message. In Y12.FOR, the system will again catch the exceeds alarm limit condition and pause. At this point, you can explain why the alarm limit was exceeded by changing the state to one of the faulty sample codes (i.e., "SAMPLE SPILLED," "CLOGGED RESIN," etc.). However, if you insist that "SAMPLE IS OK," then the state that is passed to the ddmmmyyyy.RES flat file will be "EXCEEDS."

After you have fixed the sample, edit NAMES.DAT so that you will start at this sample (or skip it if that is what you decide to do) when you resume processing.

e. Lam not sure what is wrong with the sample. I want to process it further to get more information.

After the end of the ALPHA_MDA.FOR program, the "ALPHA SPECTROSCOPY REPORT" is generated and printed. This report gives quite a bit of information about the sample. This information may help explain what is wrong with the sample. It is possible to keep the sample from showing up in the ddmmmyyyy.RES flat file by killing the processing after the report is generated.

To continue processing this sample, hit " $\mathrm{n}$ " or " $\mathrm{N}$ " at the "do you want to terminate alpha_mda right now?" message. After the "ALPHA SPECTROSCOPY REPORT" is generated, you can stop the processing with a ctrl-y. In Y12.FOR, the system will again catch the exceeds alarm limit condition and pause. This is a convenient point to stop processing because the system pauses here. Note that breaking out with a ctrl-y stops all further processing.

After you have fixed the sample or whatever else you need to do, edit NAMES.DAT so that you will start at this sample (or skip it if that is what you decide to do) when you resume processing. 


\section{APPENDIX 2.H EFFECT OF TRUNCATION ERROR ON STANDARD DEVIATION}

In some early tests of the modified ALPHA_MDA software, the author used a set of dummy reagent blank files that were created by copying a real blank file to files with names that clearly identified them as dummies. Except for their names, the files were identical. This was a test to demonstrate that the calculations for average and standard deviation in entry hblkinf were working correctly. The result of processing several identical sets of data should have been a zero value of standard deviation. Instead, some of the standard deviation calculations caused a square root of a negative number error. The other standard deviations were small, but non-zero. A explanation was wanted for the fact that the standard deviation of a series of nominally identical numbers was not zero (and, even worse, was sometimes imaginary). The answer lies in the accumulation of truncation errors in the passing of activities from the ALPHA_MDA code to the blank history file and back, plus the truncation error in the sums that are used to calculate standard deviation.

The standard deviation is as follows:

$$
\sigma=\sqrt{\left[\sum_{n=1}^{N} x_{n}^{2}-\frac{1}{N}\left(\sum_{n=1}^{N} x_{n}\right)^{2}\right] /(N-1)} .
$$

Suppose that all the $x_{n}$ are identical and equal to some value $x$. Thanks to truncation error, the true value of $x_{n}$ is not known within some percentage error $\epsilon$. In other words,

$$
x(1-\epsilon)<x_{n}<x(1+\epsilon) .
$$

If one constructs the standard deviation in a form that leads to the largest accumulation of truncation errors, the result is as follows:

$$
\sigma_{\max }=\sqrt{\left[\sum_{n=1}^{N} x^{2}(1+\epsilon)^{2}-\frac{1}{N}\left(\sum_{n=1}^{N} x(1-\epsilon)\right)^{2}\right] /(N-1)} .
$$

Doing the algebra, the value of standard deviation becomes

$$
\sigma_{\max }=2 x \sqrt{\epsilon \frac{N}{(N-1)}} .
$$

For a real*8 variable, a very approximate estimate of the truncation error is $\epsilon \approx 10^{-14}$, which gives

$$
\sigma_{\max }(x)_{\text {real } * 8} \approx 2 \times 10^{-7}
$$

For a real $* 4$ variable, a very approximate estimate of the truncation error is $\epsilon \approx 10^{-7}$, which gives 


$$
\sigma_{\max }(x)_{\text {real } * 4} \approx 6 \times 10^{-4}
$$

The truncation error for a standard deviation calculation in ALPHA_MDA is more complicated than this simple model because the activities are passed back and forth from the history file using a (g15.8) format. The rounding operations needed to write the activities to the history file and read them back again cause an additional level of truncation. Some experiments were run on a short piece of code on an IBM RS/6000 workstation to see just how truncation errors accumulate in "real" calculations. A value of $N=10$ was set and standard deviations were calculated a large number of different values of $x$. The values of $x$ were kept in real $* 8$, and standard deviations were calculated in real $* 8$. In addition, the values of $x$ were truncated to real $* 4$, passed to a disk file using the ( $\mathrm{g} 15.8$ ) format, and read back into a real*4 variable with the same format. A second standard deviation was calculated using the value read back, but accumulating the sums in real*8 arithmetic (real*8 arithmetic was used because the sums for the standard deviation in entry hblkinf are accumulated in real*8). The code calculated the maximum "truncation" error [i.e., 1 - (the value of $x$ read back from the disk file carried in real*4)/(the original value of $x$ in real*8)]. The maximum "truncation" error was about $8 \times 10^{-8}$. This would yield a value of $\sigma_{\max } x$ of about $5.7 \times 10^{-4}$. In theory, the maximum "truncation" error could have gone as high as $10 \times 10^{-8}$ which would yield a value of $\sigma_{\max } x$ of about $6.3 \times 10^{-4}$. The maximum value of $\sigma_{\max } / x$ found in the calculational experiment was $6.8 \times 10^{-4}$. This value is within $8 \%$ of the value for the theoretical maximum "truncation" error and within $20 \%$ of the theoretical value for the .actual maximum "truncation" error. As a footnote, the maximum value of standard deviation did not occur for the maximum "truncation" error. Life in the computational world is apparently not that simple.

From the theory and calculated results discussed in this appendix, it is clear that small values of standard deviation cannot be trusted. In particular, if the standard deviation is not at least $6 \times 10^{-4}$ times the activity, then the standard deviation is too low to be believable-that is, one knows that the standard deviation is small, but not precisely how small.

The background and blank standard deviations are used to calculate MDA and $\mathrm{L}_{\mathrm{c}}$. Therefore, one must conclude that the errors in MDA and $\mathrm{L}_{\mathrm{c}}$ are at least as large as those in the underlying standard deviations. Because the blank and background values are added to get $\mathrm{MDA}$ and $\mathrm{L}_{\mathrm{c}}$, the combination of errors increases. On the theory that the root of the sum of the squares of two identical errors is the square root of 2 times the error, the maximum percentage error due to "truncation" for $\mathrm{MDA}$ and $\mathrm{L}_{c}$ becomes nearly $10^{-3}\left(6 \times 10^{-4} \times 1.4\right.$ $\left.=9.5 \times 10^{-4}\right)$.

The conclusion to be drawn from this appendix is that small values of MDA and $\mathrm{L}_{c}$ should not be trusted. In particular, if MDA and $L_{c}$ are not at least $10^{-3}$ times the activity, then the values are too low to be believable - that is, one knows that they are small, but not precisely how small. Thanks to the 3/ERTA term in the expression for MDA, it is unlikely that MDA will be small enough to not be believable. Moreover, in real measurements in which the activities vary from sample to sample, the standard deviations of the blanks and backgrounds should be large compared to the threshold discussed in this appendix. Nevertheless, one must be aware that this threshold of believability does exist and should know the magnitude of the threshold. 
INTERNAL DISTRIBUTION

1. K. W. Childs

2. D. M. Hetrick

3. J. L. Hilemon

4-8. J. R. Kirkpatrick

9. M. A. Kuliasha

10-11. L. A. Lewis

12. M. M. Reichert

13-14. W. O. Tucker

15. Central Research Library

16. Laboratory Records

\section{EXTERNAL DISTRIBUTION}

17. R. J. Schlossmann, Canberra Nuclear, 150 Spring Lake Dr., Itasca, IL 60143.

18. Office of Scientific and Technical Information, P.O. Box 62, Oak Ridge, TN 37831. 